\title{
[1,2]- vs. [2,3]-Wittig Rearrangement in Carbohydrate Derived Alkenyl Systems
}

Ramu Sridhar Perali *, Umamaheswara Rao Boddu, and Drisya Chittadi Sankar School of Chemistry, University of Hyderabad, Hyderabad - 500046. e-mail: p_ramu_sridhar@uohyd.ac.in

Index:

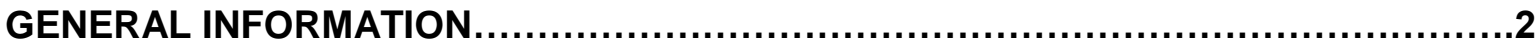

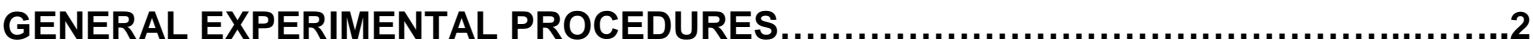

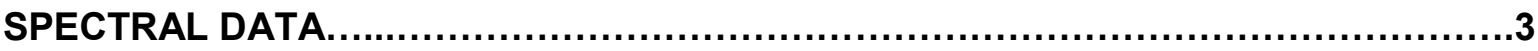

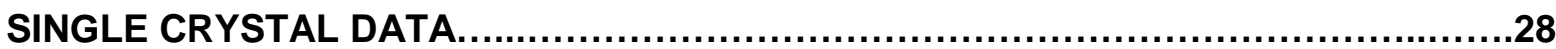

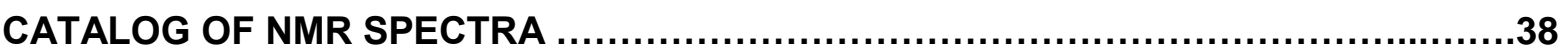




\section{General Information:}

All the reactions were carried out under nitrogen or argon atmosphere and monitored by thin layer chromatography (TLC) using silica gel $\mathrm{GF}_{254}$ plates with detection by charring with $5 \%(\mathrm{v} / \mathrm{v}) \mathrm{H}_{2} \mathrm{SO}_{4}$ in methanol or by phosphomolybdic acid (PMA) stain or by ultra violet (UV) detection. All the chemicals were purchased from local suppliers and Sigma-Aldrich Chemicals Company. Solvents used in the reactions were distilled over dehydrated agents. Silica-gel (100-200 mesh) was used for column chromatography. ${ }^{1} \mathrm{H},{ }^{13} \mathrm{C}, \mathrm{DEPT}, \mathrm{COSY}, \mathrm{NOESY}$ spectra were recorded on Bruker $400 \mathrm{MHz}$ and $500 \mathrm{MHz}$ spectrometer in $\mathrm{CDCl}_{3} .{ }^{1} \mathrm{H}$ NMR chemical shifts were reported in ppm $(\delta)$ with TMS as internal standard $(\delta 0.00)$ and ${ }^{13} \mathrm{C}$ NMR were reported in chemical shifts with solvent reference $\left(\mathrm{CDCl}_{3}, \delta 77.00\right)$. High resolution mass spectra (HRMS) were obtained in the ESI-TOF mode.

\section{General Experimental procedures:}

\section{General procedure for TBS Protection of alcohol:}

To a stirred solution of alcohol in anhydrous DMF ( $3 \mathrm{~mL} / \mathrm{mmol})$ under inert atmosphere was added imidazole (1.2 eq for each hydroxyl) followed by TBSCl (1.1 eq for each hydroxyl) at room temperature and stirring was continued for overnight. The reaction was quenched with slow addition of cold water and extracted with diethyl ether. The combined ethereal layers were washed with aqueous cupric sulphate, dried over anhydrous $\mathrm{Na}_{2} \mathrm{SO}_{4}$ and concentrated under reduced pressure to obtain crude product. Purification of the crude product by column chromatography over silica gel using hexanes and ethyl acetate provided pure O-TBS protected sugar derivatives.

\section{General procedure for Wittig Rearrangement reaction:}

A solution of Wittig precursor in anhydrous THF $(10 \mathrm{~mL} / \mathrm{mmol})$ under argon was cooled to $-78{ }^{\circ} \mathrm{C}$ and 1 eq of TMEDA was added and stirred for 5 minutes. Then a solution of $n$-BuLi (5 eq) was added and stirred at $-78^{\circ} \mathrm{C}$ for 30 minutes. Then the temperature was allowed to rise to $0^{\circ} \mathrm{C}$ over a period of 45 minutes. The reaction was quenched with water and the product was extracted with ethyl acetate. The combined organic layers were washed with brine, dried over anhydrous $\mathrm{Na}_{2} \mathrm{SO}_{4}$ and concentrated under reduced pressure to obtain crude product. Purification of the crude product by column chromatography over silica gel using hexanes and ethyl acetate provided the carbon branched sugar derivatives.

\section{General procedure for alkylation of 4,6-O-benzylidene protected glycal derivatives :}

The 4,6-benzylidene protected glycal was dissolved in anhydrous THF ( $3 \mathrm{~mL} / \mathrm{mmol})$ under an inert atmosphere and the mixture was cooled to $0^{\circ} \mathrm{C}$. $\mathrm{NaH}(1.5 \mathrm{eq}, 60 \%)$ was added portion wise with stirring over a period of 20 minutes. After continues stirring for a further 1 hour at $0^{\circ} \mathrm{C}$, alkyl halide $(1.25 \mathrm{eq})$ and TBAI $(0.1 \mathrm{eq})$ were added at $0^{\circ} \mathrm{C}$ and the mixture was stirred overnight at $25^{\circ} \mathrm{C}$. After completion of the reaction (monitored by TLC cold water was added dropwise and the obtained solution was extracted with ethyl acetate. The combined organic layer were dried over anhydrous $\mathrm{Na}_{2} \mathrm{SO}_{4}$ and concentrated under reduced pressure to obtain the crude alkyl protected glycal derivatives. 


\section{General procedure for p-methoxybenzylidene de-protection of fully protected glycal:}

A $50 \mathrm{~mL}$ RB flask containing 4,6-O-benzylidene protected glycal was dissolved in 8:1:1 AcOH: THF: $\mathrm{H}_{2} \mathrm{O}(10 \mathrm{~mL} / \mathrm{mmol})$ and heated to $45^{\circ} \mathrm{C}$ for 30 minutes (For allal derivatives the reaction was performed at room temperature). After completion of the reaction (by TLC) the reaction mixture was neutralized with sat. $\mathrm{NaHCO}_{3}$ and extracted with ethyl acetate. The combined organic layers was washed with brine, dried over anhydrous $\mathrm{Na}_{2} \mathrm{SO}_{4}$. and concentrated under reduced pressure to obtain crude product. Purification of the crude product by column chromatography over silica gel using hexanes and ethyl acetate provided the diol sugar derivatives.

\section{Spectral Data:}

(S)-1-((2R,3S,4R)-3-((tert-butyldimethylsilyl)oxy)-2-(((tert-butyldimethylsilyl)oxy) methyl)-3,4-dihydro-2H-pyran-4-yl)prop-2-en-1-ol (2)

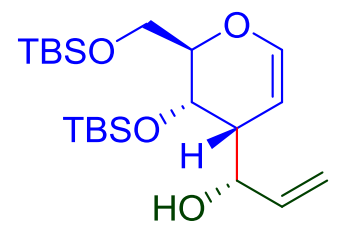

2

Yield: $90 \mathrm{mg}, 60 \%$. Rf: 0.45 (5\% EtOAc/hexane). 2: IR (neat): 2929, 2856, 2165, 1737, 1362, 1254, $1113 \mathrm{~cm}^{-1}$. ${ }^{1} \mathrm{H}$ NMR (400 MHz, $\mathbf{C D C l}_{3}$ ): $\delta 6.53$ (dd, $1 \mathrm{H}, J=1.6 \mathrm{~Hz}, J=6.0 \mathrm{~Hz}$ ), 5.85$5.93(\mathrm{~m}, 1 \mathrm{H}), 5.32(\mathrm{dt}, 1 \mathrm{H}, J=1.6 \mathrm{~Hz}, J=17.2 \mathrm{~Hz}), 5.18(\mathrm{dt}, 1 \mathrm{H}, J=1.6 \mathrm{~Hz}, J=10.8 \mathrm{~Hz})$, 4.56-4.60 (m, 1H), $4.51(\mathrm{dd}, 1 \mathrm{H}, J=4.4 \mathrm{~Hz}, J=6.0 \mathrm{~Hz}), 4.11-4.19(\mathrm{~m}, 2 \mathrm{H}), 3.78(\mathrm{t}, 2 \mathrm{H}, J=4.0$ $\mathrm{Hz}), 2.34-2.38(\mathrm{~m}, 1 \mathrm{H}), 2.24(\mathrm{~d}, 1 \mathrm{H}, J=6.8 \mathrm{~Hz}), 0.94(\mathrm{~s}, 9 \mathrm{H}), 0.91(\mathrm{~s}, 9 \mathrm{H}), 0.16(\mathrm{~s}, 3 \mathrm{H}), 0.14$ (s, 3H), 0.08 (s, 3H), 0.07 (s, 3H). $\left.{ }^{13} \mathbf{C ~ N M R ~ ( 1 0 0 ~ M H z , ~} \mathbf{C D C l}_{3}\right): \delta$ 145.7, 139.9, 114.3, 93.8, 77.2, 69.3, 66.7, 62.2, 40.3, 25.9, 25.8, 18.4, 18.0, -4.4, -4.8, -5.2, -5.3. HRMS (ESI-TOF) $\mathrm{m} / \mathrm{z}$ : $[\mathrm{M}+\mathrm{H}]^{+}$calcd for $\mathrm{C}_{21} \mathrm{H}_{43} \mathrm{O}_{4} \mathrm{Si}_{2} 415.2700$, found 415.2693.

\section{tert-butyl(((1S,3R,4S,5R,6S)-4-((tert-butyldimethylsilyl)oxy)-6-vinyl-2,7-dioxabicyclo} [3.2.1]octan-3-yl)methoxy)dimethylsilane (2a)

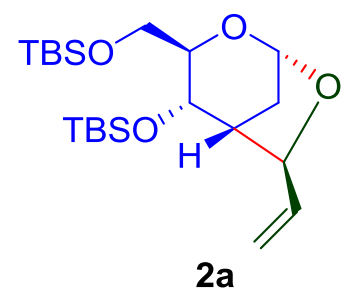

Yield: 50 mg, 90\%; Rf: 0.6 (5\% EtOAc/hexane). 2a: IR (neat): 2952, 2928, 2887, 2855, 1461, 1388, 1360, $1251 \mathrm{~cm}^{-1} .{ }^{1} \mathrm{H}$ NMR (500 MHz, $\mathbf{C D C l}_{3}$ ): $\delta 5.80$ (ddd, $1 \mathrm{H}, J=5.5 \mathrm{~Hz}, J=10.5 \mathrm{~Hz}$, $J=17.0 \mathrm{~Hz}$ ), $5.42(\mathrm{~d}, 1 \mathrm{H}, J=3.0 \mathrm{~Hz}), 5.27(\mathrm{dt}, 1 \mathrm{H}, J=1.5 \mathrm{~Hz}, J=17.0 \mathrm{~Hz}), 5.13(\mathrm{dt}, 1 \mathrm{H}, J=$ $1.5 \mathrm{~Hz}, J=10.5 \mathrm{~Hz}$ ), $4.85(\mathrm{~d}, 1 \mathrm{H}, J=5.5 \mathrm{~Hz}), 3.89$ (dd, $1 \mathrm{H}, J=3.5 \mathrm{~Hz}, J=8.5 \mathrm{~Hz}), 3.72-3.78$ $(\mathrm{m}, 2 \mathrm{H}), 3.52(\mathrm{dt}, 1 \mathrm{H}, J=2.0 \mathrm{~Hz}, J=8.5 \mathrm{~Hz}), 2.28(\mathrm{dd}, 1 \mathrm{H}, J=3.5 \mathrm{~Hz}, J=5.5 \mathrm{~Hz}$ ), 1.84 (ddd, $1 \mathrm{H}, J=3.0 \mathrm{~Hz}, J=5.5 \mathrm{~Hz}, J=11.5 \mathrm{~Hz}), 1.58(\mathrm{~d}, 1 \mathrm{H}, J=11.5 \mathrm{~Hz}), 0.91(\mathrm{~s}, 9 \mathrm{H}), 0.90(\mathrm{~s}, 9 \mathrm{H})$, $\left.0.10(\mathrm{~s}, 3 \mathrm{H}), 0.09(\mathrm{~s}, 3 \mathrm{H}), 0.07(\mathrm{~s}, 3 \mathrm{H}), 0.06(\mathrm{~s}, 3 \mathrm{H}) .{ }^{13} \mathrm{C} \mathrm{NMR} \mathrm{(125} \mathrm{MHz,} \mathbf{C D C l}_{3}\right): \delta$ 137.8, 115.2, 98.9, 78.7, 76.2, 66.3, 61.9, 45.2, 33.7, 25.9, 25.7, 18.3, 17.8, -4.9, -4.9, -5.0, -5.4. HRMS (ESI-TOF) $\mathrm{m} / z$ : [M+H] ${ }^{+}$calcd for $\mathrm{C}_{21} \mathrm{H}_{43} \mathrm{O}_{4} \mathrm{Si}_{2} 415.2700$, found 415.2691 . 
tert-butyl(((2R,3S,6S)-3-((tert-butyldimethylsilyl)oxy)-6-(prop-2-yn-1-yloxy)-3,6-di hydro-2H-pyran-2-yl)methoxy)dimethylsilane (S3)

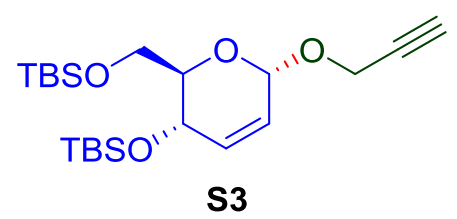

Yield: $220 \mathrm{mg}$, 98\%. Rf: 0.55 (5\% EtOAc/hexane). 3s: IR (neat): 3311, 2952, 2928, 2892, 2856, 2359, 2339, $1738 \mathrm{~cm}^{-1}$. ${ }^{1} \mathrm{H}$ NMR (500 MHz, $\left.\mathbf{C D C l}_{3}\right): \delta 5.88$ (d, $1 \mathrm{H}, J=10 \mathrm{~Hz}$ ), 5.70 (dt, $1 \mathrm{H}, J=2.5 \mathrm{~Hz}, J=10.5 \mathrm{~Hz}), 5.16(\mathrm{~s}, 1 \mathrm{H}), 4.31(\mathrm{~d}, 2 \mathrm{H}, J=2.5 \mathrm{~Hz}), 4.17(\mathrm{dq}, 1 \mathrm{H}, J=1.5 \mathrm{~Hz}, J$ $=9.0 \mathrm{~Hz}$ ), $3.87(\mathrm{dd}, 1 \mathrm{H}, J=2.0 \mathrm{~Hz}, J=11.0 \mathrm{~Hz}$ ), $3.73(\mathrm{dd}, 1 \mathrm{H}, J=6.0 \mathrm{~Hz}, J=11.0 \mathrm{~Hz}$ ), 3.66$3.70(\mathrm{~m}, 1 \mathrm{H}), 2.43(\mathrm{t}, 1 \mathrm{H}, J=2.5 \mathrm{~Hz}), 0.92(\mathrm{~s}, 9 \mathrm{H}), 0.89(\mathrm{~s}, 9 \mathrm{H}), 0.10(\mathrm{~s}, 3 \mathrm{H}), 0.09(\mathrm{~s}, 9 \mathrm{H}) .{ }^{13} \mathrm{C}$ NMR (125 MHz, $\left.\mathbf{C D C l}_{3}\right): \delta 134.9,124.9,92.3,79.5,74.3,72.8,64.0,62.5,54.3,25.9,25.6$, 18.4, 17.9, -4.3, -4.9, -5.2, -5.4. HRMS (ESI-TOF) $\mathrm{m} / \mathrm{z}$ : $[\mathrm{M}+\mathrm{Na}]^{+}$calcd for $\mathrm{C}_{21} \mathrm{H}_{40} \mathrm{O}_{4} \mathrm{Si}_{2} \mathrm{Na}$ 435.2362, found 435.2356 .

(R)-1-((2R,3S,4R)-3-((tert-butyldimethylsilyl)oxy)-2-(((tert-butyldimethylsilyl)oxy)methyl )-3,4-dihydro-2H-pyran-4-yl)prop-2-yn-1-ol (3)

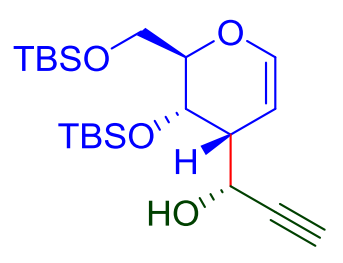

3

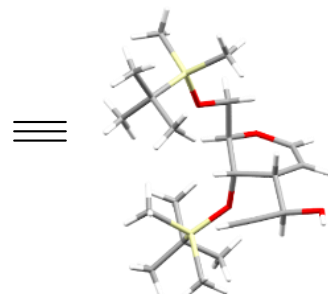

Yield: 60 mg, 60\%. $\mathrm{R}_{\mathrm{f}}$ : 0.4 (5\% EtOAc/hexane). 3: IR (neat): 3309, 2953, 2928, 2856, 1972, 1648, 1469, 1389, 1360, 1254, 1105, $1023 \mathrm{~cm}^{-1}$. ${ }^{1} \mathbf{H}$ NMR (500 MHz, $\left.\mathbf{C D C l}_{3}\right): \delta 6.59(\mathrm{dd}, 1 \mathrm{H}$, $J=1.5 \mathrm{~Hz}, J=6.0 \mathrm{~Hz}$ ), $4.77(\mathrm{dd}, 1 \mathrm{H}, J=5.0 \mathrm{~Hz}, J=6.0 \mathrm{~Hz}), 4.74(\mathrm{dt}, 1 \mathrm{H}, J=2.5 \mathrm{~Hz}, J=9.0$ $\mathrm{Hz}$ ), $4.16(\mathrm{dd}, 1 \mathrm{H}, J=5.5 \mathrm{~Hz}, J=8.0 \mathrm{~Hz}), 4.07-4.10(\mathrm{~m}, 1 \mathrm{H}), 3.78(\mathrm{t}, 2 \mathrm{H}, J=3.5 \mathrm{~Hz}), 2.51-$ $2.55(\mathrm{~m}, 1 \mathrm{H}), 2.45(\mathrm{~d}, 1 \mathrm{H}, J=2.0 \mathrm{~Hz}), 2.13(\mathrm{~d}, 1 \mathrm{H}, J=9.0 \mathrm{~Hz}), 0.93(\mathrm{~s}, 9 \mathrm{H}), 0.91(\mathrm{~s}, 9 \mathrm{H}), 0.14$ (s, 3H), 0.13 (s, 3H), 0.08 (s, 3H), 0.07(s, 3H). $\left.{ }^{13} \mathbf{C ~ N M R ~ ( 1 2 5 ~ M H z , ~ C D C l} 3\right): \delta 146.4,93.7$, 84.4, 76.9, 72.5, 65.6, 62.0, 59.9, 42.3, 25.9, 25.7, 18.4, 18.0, -4.5, -4.9, -5.2, -5.4. HRMS (ESI-TOF) $\mathrm{m} / \mathrm{z}$ : $[\mathrm{M}+\mathrm{H}]^{+}$calcd for $\mathrm{C}_{21} \mathrm{H}_{41} \mathrm{O}_{4} \mathrm{Si}_{2} 413.2545$, found 413.2538 .

(((2R,3S,6S)-6-(benzyloxy)-2-(((tert-butyldimethylsilyl)oxy)methyl)-3,6-dihydro-2Hpyran -3-yl)oxy)(tert-butyl)dimethylsilane (S4)

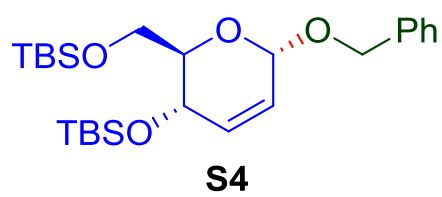

Yield: $192 \mathrm{mg}, 98 \% . \mathrm{R}_{\mathrm{f}}: 0.55$ (5\% EtOAc/hexane). S4: IR (neat): 2952, 2928, 2885, 2855, 2359, 2339, $1738 \mathrm{~cm}^{-1} .{ }^{1} \mathrm{H}$ NMR (500 MHz, $\left.\mathbf{C D C l}_{3}\right): \delta 7.29-7.43(\mathrm{~m}, 5 \mathrm{H}), 5.90(\mathrm{~d}, 1 \mathrm{H}, J=10.5$ $\mathrm{Hz}), 5.75(\mathrm{~d}, 1 \mathrm{H}, J=10 \mathrm{~Hz}), 5.10(\mathrm{~s}, 1 \mathrm{H}), 4.87(\mathrm{~d}, J=11.5 \mathrm{~Hz}), 4.62(\mathrm{~d}, 1 \mathrm{H}, J=12.0 \mathrm{~Hz})$, 4.22-4.23 (m, 1H), $3.88(\mathrm{~d}, 1 \mathrm{H}, J=9.5 \mathrm{~Hz}), 3.78-3.83(\mathrm{~m}, 2 \mathrm{H}), 0.97(\mathrm{~s}, 9 \mathrm{H}), 0.95(\mathrm{~s}, 9 \mathrm{H}), 0.14-$ $0.15(\mathrm{~m}, 12 \mathrm{H}) .{ }^{13} \mathrm{C}$ NMR (125 MHz, $\left.\mathbf{C D C l}_{3}\right): \delta 138.1,134.5,128.3,128.1,127.5,125.4,93.3$, 
72.7, 69.6, 64.1, 62.7, 25.9, 25.7, 18.4, 17.9, -4.3, -4.9, -5.1, -5.3. HRMS (ESI-TOF) $\mathrm{m} / \mathrm{z}$ : $\left[\mathrm{M}+\mathrm{NH}_{4}\right]^{+}$calcd for $\mathrm{C}_{25} \mathrm{H}_{48} \mathrm{O}_{4} \mathrm{Si}_{2} \mathrm{~N}$ 482.3122, found 482.3116 .

( $R$ )-((2R,3S,4R)-3-((tert-butyldimethylsilyl)oxy)-2-(((tert-butyldimethylsilyl)oxy) methyl)3,4-dihydro-2H-pyran-4-yl)(phenyl)methanol (4)

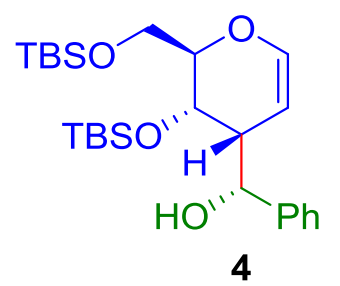

Yield: 55 mg, 55\%. Rf: 0.45 (5\% EtOAc/hexane). 4: IR (neat): 2952, 2928, 2855, $1736 \mathrm{~cm}^{-1}$. ${ }^{1} \mathrm{H}$ NMR $\left(500 \mathrm{MHz}, \mathrm{CDCl}_{3}\right)$ : $\delta 7.27-7.39(\mathrm{~m}, 5 \mathrm{H}), 6.50(\mathrm{dd}, 1 \mathrm{H}, J=1.5 \mathrm{~Hz}, J=6.0 \mathrm{~Hz}), 5.19$ (dd, $1 \mathrm{H}, J=1.5 \mathrm{~Hz}, J=5.0 \mathrm{~Hz}), 4.38(\mathrm{dd}, 1 \mathrm{H}, J=4.0 \mathrm{~Hz}, J=6.5 \mathrm{~Hz}), 4.29(\mathrm{t}, 1 \mathrm{H}, J=6.0 \mathrm{~Hz})$, 4.18-4.21 (m, $1 \mathrm{H}), 3.75-3.83(\mathrm{~m}, 2 \mathrm{H}), 2.85(\mathrm{~d}, 1 \mathrm{H}, J=5.5 \mathrm{~Hz}), 2.56-2.58(\mathrm{~m}, 1 \mathrm{H}), 1.00(\mathrm{~s}, 9 \mathrm{H})$, $0.93(\mathrm{~s}, 9 \mathrm{H}), 0.23(\mathrm{~s}, 3 \mathrm{H}), 0.21(\mathrm{~s}, 3 \mathrm{H}), 0.10(\mathrm{~s}, 3 \mathrm{H}), 0.09(\mathrm{~s}, 3 \mathrm{H}) \cdot{ }^{13} \mathbf{C} \mathbf{N M R}\left(125 \mathrm{MHz}, \mathrm{CDCl}_{3}\right)$ : $\delta$ 145.3, 143.6, 128.2, 126.9, 125.6, 93.9, 77.3, 71.0, 67.3, 62.2, 42.0, 25.9, 25.8, 18.4, 18.1, $-4.2,-4.7,-5.2,-5.3$. HRMS (ESI-TOF) $m / z$ : $[\mathrm{M}+\mathrm{Na}]^{+}$calcd for $\mathrm{C}_{25} \mathrm{H}_{44} \mathrm{O}_{4} \mathrm{Si}_{2} \mathrm{Na}$ 487.2676, found 487.2687 .

tert-butyl(((2R,3S,6S)-3-((tert-butyldimethylsilyl)oxy)-6-(cinnamyloxy)-3,6-dihydro-2Hpyran-2-yl)methoxy)dimethylsilane (S5)

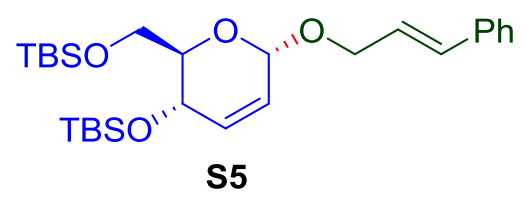

Yield: 183 mg, 98\%. Rf: 0.65 (5\% EtOAc/hexane). S5: IR (neat): 2952, 2927, 2887, $2855 \mathrm{~cm}^{-}$ 1. ${ }^{1} \mathrm{H}$ NMR (500 MHz, $\left.\mathbf{C D C l}_{3}\right): \delta$ 7.32-7.42 (m, 4H), 7.25-7.28 (m, 1H), $6.66(\mathrm{~d}, 1 \mathrm{H}, J=10.5$ $\mathrm{Hz}), 6.35(\mathrm{dt}, 1 \mathrm{H}, J=6.0 \mathrm{~Hz}, J=16.0 \mathrm{~Hz}), 5.90(\mathrm{~d}, 1 \mathrm{H}, J=10.0 \mathrm{~Hz}), 5.75(\mathrm{dt}, 1 \mathrm{H}, J=2.5 \mathrm{~Hz}$, $J=10.0 \mathrm{~Hz}$ ), $5.10(\mathrm{~s}, 1 \mathrm{H}), 4.48$ (ddd, $1 \mathrm{H}, J=1.5 \mathrm{~Hz}, J=6.0 \mathrm{~Hz}, J=13.0 \mathrm{~Hz}), 4.20-4.27(\mathrm{~m}$, $2 \mathrm{H})$, 3.90-3.93 (m, 1H), 3.77-3.81 (m, 2H), $0.96(\mathrm{~s}, 9 \mathrm{H}), 0.93(\mathrm{~s}, 9 \mathrm{H}), 0.13-0.12(\mathrm{~m}, 12 \mathrm{H}) .{ }^{13} \mathrm{C}$ NMR (125 MHz, $\left.\mathbf{C D C l}_{3}\right): \delta$ 136.8, 134.6, 132.7, 128.5, 127.6, 126.5, 125.8, 125.5, 93.3, 72.7, 68.3, 64.1, 62.7, 25.9, 25.7, 18.4, 17.9, -4.3, -4.9, -5.1, -5.3. HRMS (ESI-TOF) $\mathrm{m} / \mathrm{z}$ : $\left[\mathrm{M}+\mathrm{NH}_{4}\right]^{+}$ calcd for $\mathrm{C}_{27} \mathrm{H}_{50} \mathrm{O}_{4} \mathrm{Si}_{2} \mathrm{~N} 508.3279$, found 508.3272 .

(S,E)-1-((2R,3S,4R)-3-((tert-butyldimethylsilyl)oxy)-2-(((tert-butyldimethylsilyl)oxy) methyl)-3,4-dihydro-2H-pyran-4-yl)-3-phenylprop-2-en-1-ol (5)

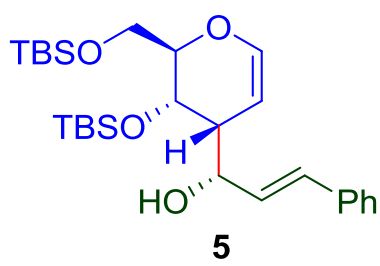

Yield: 60 mg, 60\%. Rf: 0.45 (5\% EtOAc/hexane). 5: IR (neat): 3438, 2952, 2928, 2856, 1736, $1649 \mathrm{~cm}^{-1}$. ${ }^{1} \mathrm{H}$ NMR (500 MHz, $\left.\mathbf{C D C l}_{3}\right): \delta$ 7.40-7.42 (m, 2H), 7.31-7.35 (m, 3H), $6.68(\mathrm{dd}, 1 \mathrm{H}$, $J=1.5 \mathrm{~Hz}, J=16.0 \mathrm{~Hz}$ ), 6.54 (dd, $1 \mathrm{H}, J=1.5 \mathrm{~Hz}, J=6.5 \mathrm{~Hz}), 6.24$ (dd, $1 \mathrm{H}, J=5.0 \mathrm{~Hz}, J=$ $15.5 \mathrm{~Hz}), 4.75(\mathrm{bd}, 1 \mathrm{H}, J=4.5 \mathrm{~Hz}), 4.58(\mathrm{dd}, 1 \mathrm{H}, J=4.0 \mathrm{~Hz}, J=6.0 \mathrm{~Hz}), 4.23(\mathrm{dd}, 1 \mathrm{H}, J=5.5$ 
$\mathrm{Hz}, J=6.5 \mathrm{~Hz}), 4.14-4.17(\mathrm{~m}, 1 \mathrm{H}), 3.78-3.81(\mathrm{~m}, 3 \mathrm{H}), 2.45-2.47(\mathrm{~m}, 1 \mathrm{H}), 0.97(\mathrm{~s}, 9 \mathrm{H}), 0.92(\mathrm{~s}$, 9H), 0.18 (s, 6H), 0.09 (s, 3H), 0.08 (s, 3H). $\left.{ }^{13} \mathbf{C ~ N M R ~ ( 1 2 5 ~ M H z , ~} \mathbf{C D C l}_{3}\right)$ : $\delta$ 145.6, 136.9, 131.4, 129.7, 128.5, 127.4, 126.4, 94.2, 77.3, 69.6, 66.9, 62.3, 40.6, 25.9, 25.8, 18.4, 18.1, -4.3, -4.8, -5.2, -5.3. HRMS (ESI-TOF) $\mathrm{m} / \mathrm{z}$ : $\left[\mathrm{M}+\mathrm{NH}_{4}\right]^{+}$calcd for $\mathrm{C}_{27} \mathrm{H}_{50} \mathrm{O}_{4} \mathrm{Si}_{2} \mathrm{~N} 508.3279$, found 508.3275.

tert-butyl(((2R,3S,6S)-3-((tert-butyldimethylsilyl)oxy)-6-((4-methylbenzyl)oxy)-3,6-di hydro-2H-pyran-2-yl)methoxy)dimethylsilane (S6)

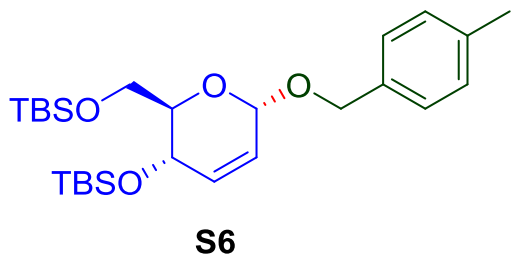

Yield: $187 \mathrm{mg}, 98 \%$. Rf: 0.65 (5\% EtOAc/hexane). S6: IR (neat): 2952, 2927, 2885, 2855, 2359, 2339, $1516 \mathrm{~cm}^{-1} .{ }^{1} \mathrm{H}$ NMR $\left(500 \mathrm{MHz}, \mathbf{C D C l}_{3}\right): \delta 7.31(\mathrm{~d}, 2 \mathrm{H}, J=8.0 \mathrm{~Hz}), 7.18(\mathrm{~d}, 2 \mathrm{H}, J$ $=8.0 \mathrm{~Hz}), 5.88(\mathrm{~d}, 1 \mathrm{H}, J=10.0 \mathrm{~Hz}), 5.72(\mathrm{dt}, 1 \mathrm{H}, J=2.5 \mathrm{~Hz}, J=10.0 \mathrm{~Hz}), 5.07-5.08(\mathrm{~m}, 1 \mathrm{H})$, $4.82(\mathrm{~d}, 1 \mathrm{H}, J=11.5 \mathrm{~Hz}), 4.58(\mathrm{~d}, 1 \mathrm{H}, J=11.5 \mathrm{~Hz}), 4.21(\mathrm{dd}, 1 \mathrm{H}, J=1.5 \mathrm{~Hz}, J=8.5 \mathrm{~Hz}), 3.86$ $3.89(\mathrm{~m}, 1 \mathrm{H}), 3.78-3.82(\mathrm{~m}, 2 \mathrm{H}), 2.38(\mathrm{~s}, 3 \mathrm{H}), 0.97(\mathrm{~s}, 9 \mathrm{H}), 0.93(\mathrm{~s}, 9 \mathrm{H}), 0.15(\mathrm{~s}, 3 \mathrm{H}), 0.14(\mathrm{~s}$, $3 \mathrm{H}), 0.13(\mathrm{~s}, 3 \mathrm{H}), 0.13(\mathrm{~s}, 3 \mathrm{H}) .{ }^{13} \mathrm{C}$ NMR (125 MHz, $\left.\mathbf{C D C l}_{3}\right): \delta 137.2,135.0,134.5,128.9$, 128.2, 125.5, 93.1, 72.7, 69.5, 64.1, 62.7, 25.9, 25.7, 21.1, 18.5, 17.9, -4.3, -4.9, -5.1, -5.3. HRMS (ESI-TOF) $\mathrm{m} / \mathrm{z}$ : $[\mathrm{M}+\mathrm{H}]^{+}$calcd for $\mathrm{C}_{26} \mathrm{H}_{47} \mathrm{O}_{4} \mathrm{Si}_{2} 479.3013$, found 479.3008 .

(R)-((2R,3S,4R)-3-((tert-butyldimethylsilyl)oxy)-2-(((tert-butyldimethylsilyl)oxy) methyl)3,4-dihydro-2H-pyran-4-yl)(p-tolyl)methanol (6)

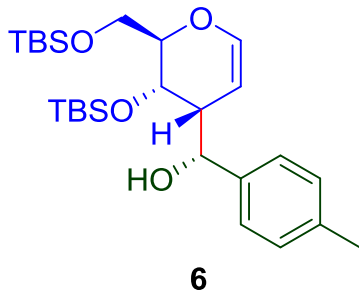

Yield: 45 mg, 45\%. Rf: 0.35 (5\% EtOAc/hexane). 6: IR (neat): 2952, 2927, 2855, 1736, 1649, $1513 \mathrm{~cm}^{-1}$. ${ }^{1} \mathrm{H}$ NMR (400 MHz, $\left.\mathbf{C D C l}_{3}\right): \delta 7.25(\mathrm{~d}, 2 \mathrm{H}, J=8.0 \mathrm{~Hz}), 7.17(\mathrm{~d}, 2 \mathrm{H}, J=8.0 \mathrm{~Hz})$, $6.49(\mathrm{dd}, 1 \mathrm{H}, J=2.0 \mathrm{~Hz}, J=16.0 \mathrm{~Hz}), 5.15(\mathrm{~d}, 1 \mathrm{H}, J=4.4 \mathrm{~Hz}), 4.39(\mathrm{dd}, 1 \mathrm{H}, J=4.0 \mathrm{~Hz}, J=$ $6.4 \mathrm{~Hz}, \mathrm{H} 2), 4.25-4.28(\mathrm{~m}, 1 \mathrm{H}), 4.17-4.21(\mathrm{~m}, 1 \mathrm{H}), 3.73-3.82(\mathrm{~m}, 2 \mathrm{H}), 2.80(\mathrm{~d}, 1 \mathrm{H}, J=5.2 \mathrm{~Hz})$, 2.53-2.56 (m, 1H), $2.36(\mathrm{~s}, 3 \mathrm{H}), 0.99(\mathrm{~s}, 9 \mathrm{H}), 0.92(\mathrm{~s}, 9 \mathrm{H}), 0.22(\mathrm{~s}, 3 \mathrm{H}), 0.20(\mathrm{~s}, 3 \mathrm{H}), 0.09(\mathrm{~s}$, 3H), 0.08 (s, 3H). ${ }^{13} \mathbf{C}$ NMR (100 MHz, $\mathbf{C D C l}_{3}$ ): $\delta$ 145.2, 140.6, 136.5, 128.9, 125.5, 94.0, 77.3, 70.9, 67.3, 62.2, 42.0, 25.9, 25.8, 21.0, 18.4, 18.1, -4.2, -4.7, -5.2, -5.3. HRMS (ESI-TOF) $\mathrm{m} / \mathrm{z}$ : $[\mathrm{M}+\mathrm{H}]^{+}$calcd for $\mathrm{C}_{26} \mathrm{H}_{47} \mathrm{O}_{4} \mathrm{Si}_{2} 479.3013$, found 479.3008 .

tert-butyl(((1S,3R,4S,5R,6R)-4-((tert-butyldimethylsilyl)oxy)-6-(p-tolyl)-2,7-dioxabicyclo [3.2.1]octan-3-yl)methoxy)dimethylsilane (6a)

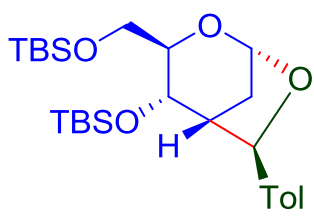

$6 a$ 
Yield: 19 mg, 95\%, Rf: 0.65 (5\% EtOAc/hexane). 6a: IR (neat): 2952, 2927, 2891, 2855, 2359, 2328, 1725, $1513 \mathrm{~cm}^{-1}$. ${ }^{1} \mathrm{H}$ NMR (500 MHz, $\left.\mathbf{C D C l}_{3}\right): \delta 7.17(\mathrm{~s}, 4 \mathrm{H}), 5.60(\mathrm{~d}, 1 \mathrm{H}, J=3.0 \mathrm{~Hz})$, $5.48(\mathrm{~s}, 1 \mathrm{H}), 3.95(\mathrm{dd}, 1 \mathrm{H}, J=3.5 \mathrm{~Hz}, J=8.5 \mathrm{~Hz}), 3.77-3.83(\mathrm{~m}, 2 \mathrm{H}), 3.63(\mathrm{dt}, 1 \mathrm{H}, J=1.5 \mathrm{~Hz}$, $J=8.5 \mathrm{~Hz}$ ), 2.41 (dd, $1 \mathrm{H}, J=3.5 \mathrm{~Hz}, J=5.0 \mathrm{~Hz}$ ), $2.35(\mathrm{~s}, 3 \mathrm{H}), 1.85$ (ddd, $1 \mathrm{H}, J=3.5 \mathrm{~Hz}, J=$ $5.5 \mathrm{~Hz}, J=12.0 \mathrm{~Hz}), 1.54(\mathrm{~d}, 1 \mathrm{H}, J=11.5 \mathrm{~Hz}), 0.98(\mathrm{~s}, 9 \mathrm{H}), 0.92(\mathrm{~s}, 9 \mathrm{H}), 0.15(\mathrm{~s}, 3 \mathrm{H}), 0.14$ (s, 3H), 0.09 (s, 3H), 0.08 (s, 3H). ${ }^{13} \mathbf{C}$ NMR (125 MHz, $\left.\mathbf{C D C l}_{3}\right): \delta 139.4,136.4,128.9,124.9$, 99.3, 79.2, 76.4, 66.6, 61.8, 48.2, 33.1, 25.9, 25.8, 21.0, 18.4, 17.9, -4.1, -5.0, -5.1, -5.4. HRMS (ESI-TOF) $\mathrm{m} / \mathrm{z}$ : [M+K] ${ }^{+}$calcd for $\mathrm{C}_{26} \mathrm{H}_{46} \mathrm{O}_{4} \mathrm{Si}_{2} \mathrm{~K}$ 517.2572, found 517.2570.

(2R,3S,6S)-6-(allyloxy)-3-methoxy-2-(methoxymethyl)-3,6-dihydro-2H-pyran (S7)

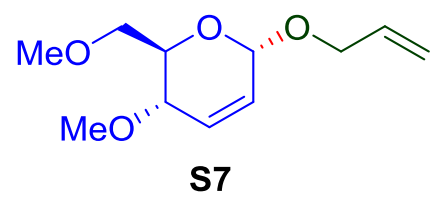

Yield: $113 \mathrm{mg}$, 99\%. Rf: 0.25 (10\% EtOAc/hexane). S7: IR (neat): 2934, 2892, 2835, $1647 \mathrm{~cm}^{-}$ 1. ${ }^{1} \mathrm{H}$ NMR $\left(500 \mathrm{MHz}, \mathrm{CDCl}_{3}\right): \delta 6.07(\mathrm{~d}, 1 \mathrm{H}, J=10.5 \mathrm{~Hz}), 5.88-5.96(\mathrm{~m}, 1 \mathrm{H}), 5.76-5.78(\mathrm{~m}$, $1 \mathrm{H}), 5.26-5.30(\mathrm{~m}, 1 \mathrm{H}), 5.15-5.17(\mathrm{~m}, 1 \mathrm{H}), 5.03(\mathrm{bs}, 1 \mathrm{H}), 4.24-4.28(\mathrm{~m}, 1 \mathrm{H}), 4.03-4.07(\mathrm{~m}, 1 \mathrm{H})$, 3.88-3.90 $(\mathrm{m}, 1 \mathrm{H}), 3.83-3.86(\mathrm{~m}, 1 \mathrm{H}), 3.59-3.65(\mathrm{~m}, 2 \mathrm{H}), 3.42(\mathrm{~s}, 3 \mathrm{H}), 3.39(\mathrm{~s}, 3 \mathrm{H}) .{ }^{13} \mathrm{C}$ NMR (125 MHz, $\mathrm{CDCl}_{3}$ ): $\delta 134.4,130.2,126.5,116.9$, 93.8, 71.8, 71.5, 68.9, 68.7, 59.2, 56.4. HRMS (ESI-TOF) $\mathrm{m} / \mathrm{z}$ : [M+Na] ${ }^{+}$calcd for $\mathrm{C}_{11} \mathrm{H}_{18} \mathrm{O}_{4} \mathrm{Na} 237.1103$, found 237.1119.

(S)-1-((2R,3S,4R)-3-methoxy-2-(methoxymethyl)-3,4-dihydro-2H-pyran-4-yl)prop-2-en1-ol (7)

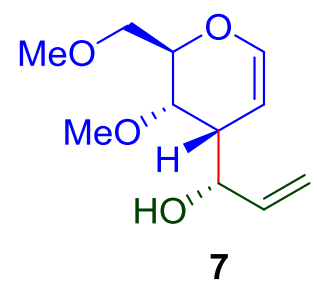

Yield: 72 mg, 72\%. Rf: 0.25 (20\% EtOAc/hexane). 7: IR (neat): 3068, 2934, 2892, 2835, 1647 $\mathrm{cm}^{-1}$. ${ }^{1} \mathrm{H}$ NMR $\left(500 \mathrm{MHz}, \mathrm{CDCl}_{3}\right): \delta 6.48(\mathrm{dd}, 1 \mathrm{H}, J=1.5 \mathrm{~Hz}, J=6.0 \mathrm{~Hz}), 5.90$ (ddd, $1 \mathrm{H}, J=$ $5.0 \mathrm{~Hz}, J=10.5 \mathrm{~Hz}, J=17.0 \mathrm{~Hz}$ ), $5.33(\mathrm{dt}, 1 \mathrm{H}, J=1.5 \mathrm{~Hz}, J=17.0 \mathrm{~Hz}), 5.20(\mathrm{dt}, 1 \mathrm{H}, J=1.5$ $\mathrm{Hz}, J=10.5 \mathrm{~Hz}$ ), $4.63(\mathrm{dd}, 1 \mathrm{H}, J=3.5 \mathrm{~Hz}, J=6.0 \mathrm{~Hz}$ ), $4.44(\mathrm{bs}, 1 \mathrm{H}), 4.37-4.40(\mathrm{~m}, 1 \mathrm{H}), 3.64$ (t, $1 \mathrm{H}, J=4.0 \mathrm{~Hz}), 3.58-3.61(\mathrm{~m}, 1 \mathrm{H}), 3.49-3.52(\mathrm{~m}, 1 \mathrm{H}), 3.46(\mathrm{~s}, 3 \mathrm{H}), 3.40(\mathrm{~s}, 3 \mathrm{H}), 2.52(\mathrm{bs}$, 1H), 2.44-2.47 (m, 1H). ${ }^{13} \mathrm{C}$ NMR (125 MHz, $\left.\mathbf{C D C l}_{3}\right): \delta$ 144.5, 139.4, 114.9, 95.4, 75.7, 72.8, 71.5, 70.7, 59.3, 57.3, 37.6. HRMS (ESI-TOF) $\mathrm{m} / \mathrm{z}$ : $[\mathrm{M}+\mathrm{Na}]^{+}$calcd for $\mathrm{C}_{11} \mathrm{H}_{18} \mathrm{O}_{4} \mathrm{Na} 237.1103$, found 237.1107 .

(((2R,3R,6S)-6-(allyloxy)-2-(((tert-butyldimethylsilyl)oxy)methyl)-3,6-dihydro-2H-pyran3-yl)oxy)(tert-butyl)dimethylsilane (S8)<smiles>C=CCO[C@@H]1C=CC(O[AsH3])[C@@H](COC(C)(F)F)O1</smiles>

S8 
Yield: $217 \mathrm{mg}, 98 \%$. Rf: 0.5 (5\% EtOAc/hexane). S8: IR (neat): 2952, 2928, 2885, $2856 \mathrm{~cm}^{-1}$. ${ }^{1} \mathrm{H}$ NMR (500 MHz, $\left.\mathrm{CDCl}_{3}\right): \delta 6.00-6.04(\mathrm{~m}, 1 \mathrm{H}), 5.92-5.99(\mathrm{~m}, 1 \mathrm{H}), 5.89(\mathrm{dd}, 1 \mathrm{H}, J=3.0 \mathrm{~Hz}$, $J=10.0 \mathrm{~Hz}), 5.29(\mathrm{dq}, 1 \mathrm{H}, J=1.5 \mathrm{~Hz}, J=17.5 \mathrm{~Hz}), 5.18(\mathrm{dq}, 1 \mathrm{H}, J=1.5 \mathrm{~Hz}, J=10.5 \mathrm{~Hz})$, $5.08(\mathrm{~d}, 1 \mathrm{H}, J=2.5 \mathrm{~Hz}), 4.29$ (tdd, $1 \mathrm{H}, J=1.5 \mathrm{~Hz}, J=5.5 \mathrm{~Hz}, J=12.5 \mathrm{~Hz}), 4.07$ (tdd, $1 \mathrm{H}, J=$ $1.5 \mathrm{~Hz}, J=6.5 \mathrm{~Hz}, J=13.0 \mathrm{~Hz}$ ), 3.98-4.01 (m, $1 \mathrm{H}), 3.91(\mathrm{dd}, 1 \mathrm{H}, J=2.5 \mathrm{~Hz}, J=5.0 \mathrm{~Hz}), 3.83$ (dd, $1 \mathrm{H}, J=5.5 \mathrm{~Hz}, J=10.5 \mathrm{~Hz}$ ), 3.77 (dd, $1 \mathrm{H}, J=7.0 \mathrm{~Hz}, J=10.5 \mathrm{~Hz}$ ), 0.92 (s, $9 \mathrm{H}$ ), 0.90 (s, 9H), 0.10 (s, 3H), 0.09 (s, 6H), 0.08 (s, 3H). ${ }^{13} \mathbf{C ~ N M R ~ ( 1 2 5 ~ M H z , ~} \mathbf{C D C l}_{3}$ ): $\delta$ 134.5, 129.8, 127.8, 117.3, 93.2, 71.8, 68.4, 62.8, 62.0, 25.9, 25.8, 18.3, 18.2, -4.0, -4.7, -5.3, -5.4. HRMS (ESI-TOF) $\mathrm{m} / \mathrm{z}$ : $[\mathrm{M}+\mathrm{K}]^{+}$calcd for $\mathrm{C}_{21} \mathrm{H}_{42} \mathrm{O}_{4} \mathrm{Si}_{2} \mathrm{~K}$ 453.2258, found 453.2250 .

(R)-1-((2R,3R,4R)-3-((tert-butyldimethylsilyl)oxy)-2-(((tert-butyldimethylsilyl)oxy) methyl)-3,4-dihydro-2H-pyran-4-yl)prop-2-en-1-ol (8a)

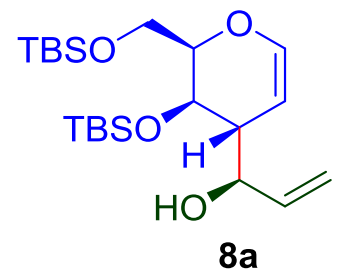

Yield: 35 mg, 35\%, $R_{\mathrm{f}}$ : 0.4 (5\% EtOAc/hexane). 8a: IR (neat): 2953, 2928, 2885, 2856, 1647 $\mathrm{cm}^{-1} .{ }^{1} \mathrm{H}$ NMR $\left(500 \mathrm{MHz}, \mathrm{CDCl}_{3}\right): \delta 6.48(\mathrm{dd}, 1 \mathrm{H}, J=1.5 \mathrm{~Hz}, J=6.0 \mathrm{~Hz}), 5.87-5.93(\mathrm{~m}, 1 \mathrm{H})$, $5.30(\mathrm{dt}, 1 \mathrm{H}, J=1.5 \mathrm{~Hz}, J=17.0 \mathrm{~Hz}), 5.25(\mathrm{dt}, 1 \mathrm{H}, J=1.5 \mathrm{~Hz}, J=10.5 \mathrm{~Hz}), 4.56(\mathrm{dd}, 1 \mathrm{H}, J=$ $3.5 \mathrm{~Hz}, J=6.0 \mathrm{~Hz}), 4.14-4.15(\mathrm{~m}, 1 \mathrm{H}), 4.06(\mathrm{dd}, 1 \mathrm{H}, \mathrm{J}=3.5 \mathrm{~Hz}, \mathrm{~J}=5.0 \mathrm{~Hz}), 3.95-3.98(\mathrm{~m}$, $1 \mathrm{H})$, 3.78-3.80 (m, 2H), 2.15-2.18 (m, 1H), $1.64(\mathrm{~d}, 1 \mathrm{H}, J=7.0 \mathrm{~Hz}), 0.91(\mathrm{~s}, 18 \mathrm{H}), 0.17(\mathrm{~s}, 3 \mathrm{H})$, 0.15 (s, 3H), $0.08(\mathrm{~s}, 3 \mathrm{H}), 0.07(\mathrm{~s}, 3 \mathrm{H}) .{ }^{13} \mathbf{C}$ NMR (125 MHz, $\left.\mathbf{C D C l}_{3}\right): \delta$ 144.9, 139.4, 115.5, 95.8, 77.3, 72.8, 66.0, 60.7, 43.6, 25.9, 25.7, 18.3, 17.9, -4.5, -4.7, -5.2, -5.4. HRMS (ESITOF) $\mathrm{m} / \mathrm{z}$ : $[\mathrm{M}+\mathrm{H}]^{+}$calcd for $\mathrm{C}_{21} \mathrm{H}_{43} \mathrm{O}_{4} \mathrm{Si}_{2} 415.2700$, found 415.2695 .

(S)-1-((2R,3R,4R)-3-((tert-butyldimethylsilyl)oxy)-2-(((tert-butyldimethylsilyl)oxy) methyl)-3,4-dihydro-2H-pyran-4-yl)prop-2-en-1-ol (8b)

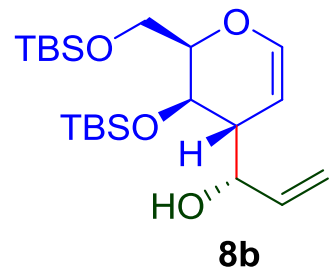

Yield: 35 mg, 35\%. Rf: 0.37 (5\% EtOAc/hexane). 8b: IR (neat): 2954, 2928, 2886, 2856, 2158, 2036, 1737, $1648 \mathrm{~cm}^{-1}$. ${ }^{1} \mathrm{H}$ NMR $\left(500 \mathrm{MHz}, \mathbf{C D C l}_{3}\right): \delta 6.46$ (dd, $\left.1 \mathrm{H}, J=1.5 \mathrm{~Hz}, J=6.0 \mathrm{~Hz}\right)$, 5.87-5.94 (m, 1H), $5.31(\mathrm{dt}, 1 \mathrm{H}, J=1.5 \mathrm{~Hz}, J=17.5 \mathrm{~Hz}), 5.24(\mathrm{dt}, 1 \mathrm{H}, J=1.0 \mathrm{~Hz}, J=10.0$ $\mathrm{Hz}), 4.52-4.55(\mathrm{~m}, 1 \mathrm{H}), 4.15-4.16(\mathrm{~m}, 1 \mathrm{H}), 3.98(\mathrm{t}, 1 \mathrm{H}, J=6.5 \mathrm{~Hz}), 3.82-3.86(\mathrm{~m}, 1 \mathrm{H}), 3.76-$ $3.80(\mathrm{~m}, 2 \mathrm{H}), 2.10-2.14(\mathrm{~m}, 1 \mathrm{H}), 1.83(\mathrm{~s}, 1 \mathrm{H}), 0.91(\mathrm{~s}, 9 \mathrm{H}), 0.90(\mathrm{~s}, 9 \mathrm{H}), 0.11(\mathrm{~s}, 3 \mathrm{H}), 0.10(\mathrm{~s}$, $3 \mathrm{H}), 0.08(\mathrm{~s}, 6 \mathrm{H}),{ }^{13} \mathrm{C}$ NMR (125 MHz, $\mathbf{C D C l}_{3}$ ): $\delta 144.2,138.3,116.6,97.8,76.3,75.2,65.3$, 61.8, 44.6, 25.9, 25.8, 18.3, 17.9, -4.5, -4.5, -5.2, -5.3. HRMS (ESI-TOF) $\mathrm{m} / \mathrm{z}$ : $[\mathrm{M}+\mathrm{H}]^{+}$calcd for $\mathrm{C}_{21} \mathrm{H}_{43} \mathrm{O}_{4} \mathrm{Si}_{2} 415.2700$, found 415.2695. 
tert-butyl (((2R,3S,6S)-3-((tert-butyldimethylsilyl)oxy)-6-(prop-2-yn-1-yloxy)-3,6-dihydro $-2 H$-pyran-2-yl)methoxy)dimethylsilane (S9)

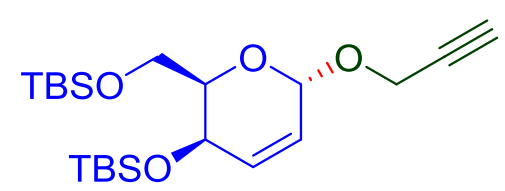

S9

Yield: $217 \mathrm{mg}$, 97\%. Rf: 0.55 (5\% EtOAc/hexane). S9: IR (neat): 3310, 2953, 2928, 2886, $2856 \mathrm{~cm}^{-1}$. ${ }^{1} \mathrm{H}$ NMR $\left(500 \mathrm{MHz}, \mathrm{CDCl}_{3}\right): \delta 6.05$ (ddd, $1 \mathrm{H}, J=1.0 \mathrm{~Hz}, J=5.5 \mathrm{~Hz} J=10.0 \mathrm{~Hz}$ ), $5.89(\mathrm{dd}, 1 \mathrm{H}, J=2.5 \mathrm{~Hz}, J=10.0 \mathrm{~Hz}), 5.25(\mathrm{~d}, 1 \mathrm{H}, J=3.0 \mathrm{~Hz}), 4.31(\mathrm{t}, 2 \mathrm{H}, J=2.0 \mathrm{~Hz}), 3.93-$ $3.96(\mathrm{~m}, 1 \mathrm{H}), 3.90-3.92(\mathrm{~m}, 1 \mathrm{H}), 3.75-3.83(\mathrm{~m}, 2 \mathrm{H}), 2.43(\mathrm{t}, 1 \mathrm{H}, J=2.5 \mathrm{~Hz}), 0.91(\mathrm{~s}, 9 \mathrm{H}), 0.89$ (s, 9H), 0.09 (s, 6H), 0.08 (s, 6H). ${ }^{13} \mathrm{C}$ NMR (125 MHz, $\left.\mathbf{C D C l}_{3}\right): \delta 130.2,127.4,92.2,79.5$, 74.3, 72.2, 62.7, 61.9, 53.9, 25.9, 25.8, 18.3, 18.1, -4.0, -4.7, -5.3, -5.4. HRMS (ESI-TOF) $\mathrm{m} / \mathrm{z}$ : $[\mathrm{M}+\mathrm{K}]^{+}$calcd for $\mathrm{C}_{21} \mathrm{H}_{40} \mathrm{O}_{4} \mathrm{Si}_{2} \mathrm{~K} 451.2103$, found 451.2092.

(S)-1-((2R,3R,4R)-3-((tert-butyldimethylsilyl)oxy)-2-(((tert-butyldimethylsilyl)oxy) methyl)-3,4-dihydro-2H-pyran-4-yl)prop-2-yn-1-ol (5)

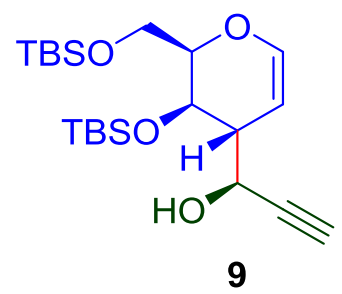

Yield: 52 mg, 50\%. Rf: 0.45 (5\% EtOAc/hexane). 9: IR (neat): 3308, 2953, 2928, 2856, 1972, $1648 \mathrm{~cm}^{-1}$. ${ }^{1} \mathrm{H}$ NMR (400 MHz, $\left.\mathrm{CDCl}_{3}\right): \delta 6.50$ (dd, $\left.1 \mathrm{H}, J=1.6 \mathrm{~Hz}, J=6.0 \mathrm{~Hz}\right), 4.72(\mathrm{ddd}, 1 \mathrm{H}$, $J=0.8 \mathrm{~Hz}, J=4.0 \mathrm{~Hz}, J=6.0 \mathrm{~Hz}), 4.31-4.32(\mathrm{~m}, 1 \mathrm{H}), 4.20(\mathrm{t}, 1 \mathrm{H}, J=2.8 \mathrm{~Hz}), 3.88(\mathrm{td}, 1 \mathrm{H}, \mathrm{J}$ $=2.4 \mathrm{H}, J=6.0 \mathrm{~Hz}$ ), $3.81(\mathrm{dd}, 2 \mathrm{H}, J=2.4 \mathrm{~Hz}, J=6.0 \mathrm{~Hz}), 2.55(\mathrm{~d}, 1 \mathrm{H}, J=2.0 \mathrm{~Hz}), 2.29-2.33$ $(\mathrm{m}, 1 \mathrm{H}), 2.02(\mathrm{~d}, 1 \mathrm{H}, J=3.2 \mathrm{~Hz}), 0.92(\mathrm{~s}, 9 \mathrm{H}), 0.91(\mathrm{~s}, 9 \mathrm{H}), 0.13(\mathrm{~s}, 3 \mathrm{H}), 0.13(\mathrm{~s}, 3 \mathrm{H}), 0.09$ (s, 3H), 0.09(s, 3H). ${ }^{13} \mathrm{C}$ NMR (100 MHz, $\left.\mathrm{CDCl}_{3}\right)$ : $\delta 144.6,96.8,82.6,76.5,74.7,65.4,64.4,61.4$, 44.6, 25.9, 25.7, 18.3, 17.9, -4.6, -4.6, -5.2, -5.3. HRMS (ESI-TOF) $\mathrm{m} / \mathrm{z}$ : $[\mathrm{M}+\mathrm{Na}]^{+}$calcd for $\mathrm{C}_{21} \mathrm{H}_{40} \mathrm{O}_{4} \mathrm{Si}_{2} \mathrm{Na}$ 435.2363, found 435.2357.

(((2S,3R,6R)-6-(allyloxy)-2-methyl-3,6-dihydro-2H-pyran-3-yl)oxy)(tert-butyl)dimethyl silane (S10)<smiles>C=CCO[C@H]1C=C[C@@H](O[AsH3])[C@H](C)O1</smiles>

Yield: $164 \mathrm{mg}, 98 \%$. Rf: 0.65 (5\% EtOAc/hexane). S10: IR (neat): 2953, 2929, 2857, $1728 \mathrm{~cm}^{-}$ 1. ${ }^{1} \mathrm{H}$ NMR (500 MHz, $\left.\mathbf{C D C l}_{3}\right): \delta 5.90-5.98(\mathrm{~m}, 1 \mathrm{H}), 5.84(\mathrm{~d}, 1 \mathrm{H}, J=10.0 \mathrm{~Hz}), 5.68(\mathrm{dt}, 1 \mathrm{H}, J=$ $2.5 \mathrm{~Hz}, J=10.5 \mathrm{~Hz}$ ), $5.30(\mathrm{dq}, 1 \mathrm{H}, J=1.5 \mathrm{~Hz}, J=17.5 \mathrm{~Hz}), 5.18(\mathrm{dq}, 1 \mathrm{H}, J=1.5 \mathrm{~Hz}, J=10.5$ $\mathrm{Hz}$ ), 4.96 (s, 1H), 4.25 (ddt, $1 \mathrm{H}, J=1.5 \mathrm{~Hz}, J=5.5 \mathrm{~Hz}, J=13.0 \mathrm{~Hz}$ ), 4.04 (ddt, $1 \mathrm{H}, J=1.5$ $\mathrm{Hz}, J=6.5 \mathrm{~Hz}, J=13.0 \mathrm{~Hz}), 3.87(\mathrm{dq}, 1 \mathrm{H}, J=1.5 \mathrm{~Hz}, J=9.0 \mathrm{~Hz}), 3.75-3.80(\mathrm{~m}, 1 \mathrm{H}), 1.25(\mathrm{~d}$, $3 \mathrm{H}, J=6.5 \mathrm{~Hz}), 0.90(\mathrm{~s}, 9 \mathrm{H}), 0.09(\mathrm{~s}, 3 \mathrm{H}), 0.08(\mathrm{~s}, 3 \mathrm{H}) .{ }^{13} \mathrm{C} \mathbf{N M R}\left(125 \mathrm{MHz}, \mathbf{C D C l}_{3}\right): \delta$ 134.7, 
134.6, 125.4, 116.9, 93.7, 70.1, 68.8, 67.7, 25.7, 18.1, 17.9, -4.3, -4.8. HRMS (ESI-TOF) $\mathrm{m} / \mathrm{z}$ : $[\mathrm{M}+\mathrm{Na}]^{+}$calcd for $\mathrm{C}_{15} \mathrm{H}_{28} \mathrm{O}_{3} \mathrm{SiNa} 307.1706$, found 307.1692.

(R)-1-((2S,3R,4S)-3-((tert-butyldimethylsilyl)oxy)-2-methyl-3,4-dihydro-2H-pyran-4-yl) prop-2-en-1-ol (10)

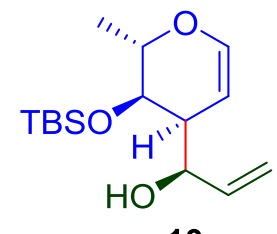

10

Yield: $66 \mathrm{mg}, 66 \%$. Rf: 0.3 (5\% EtOAc/hexane). 10: IR (neat): 3445, 2953, 2929, 2857, 1728 $\mathrm{cm}^{-1} .{ }^{1} \mathrm{H}$ NMR $\left(400 \mathrm{MHz}, \mathrm{CDCl}_{3}\right): \delta 6.50$ (dd, $\left.1 \mathrm{H}, J=1.6 \mathrm{~Hz}, J=6.0 \mathrm{~Hz}\right), 5.89$ (ddd, $1 \mathrm{H}, J=$ $4.8 \mathrm{~Hz}, J=10.4 \mathrm{~Hz}, J=17.2 \mathrm{~Hz}$ ), $5.31(\mathrm{dt}, 1 \mathrm{H}, J=1.6 \mathrm{~Hz}, J=17.2 \mathrm{~Hz}), 5.18(\mathrm{dt}, 1 \mathrm{H}, J=1.6$ $\mathrm{Hz}, J=10.8 \mathrm{~Hz}), 4.56-4.60(\mathrm{~m}, 1 \mathrm{H}), 4.53(\mathrm{dd}, 1 \mathrm{H}, J=4.0 \mathrm{~Hz}, J=6.4 \mathrm{~Hz}), 4.18-4.25(\mathrm{~m}, 1 \mathrm{H})$, $3.79(\mathrm{dd}, 1 \mathrm{H}, J=5.6 \mathrm{~Hz}, J=7.6 \mathrm{~Hz}), 2.35-2.38(\mathrm{~m}, 1 \mathrm{H}), 2.18(\mathrm{~d}, 1 \mathrm{H}, J=6.0 \mathrm{~Hz}), 1.25(\mathrm{~d}, 3 \mathrm{H}$, $J=6.4 \mathrm{~Hz}) 0.94(\mathrm{~s}, 9 \mathrm{H}), 0.14(\mathrm{~s}, 3 \mathrm{H}), 0.13(\mathrm{~s}, 3 \mathrm{H}) .{ }^{13} \mathrm{C} \mathrm{NMR}\left(100 \mathrm{MHz}, \mathrm{CDCl}_{3}\right): \delta 145.6,139.9$, 114.2, 94.3, 72.9, 72.2, 69.3, 40.8, 25.7, 18.1, 18.0, -4.3, -4.7. HRMS (ESI-TOF) $\mathrm{m} / \mathrm{z}:$ [M+Na] $^{+}$ calcd for $\mathrm{C}_{15} \mathrm{H}_{28} \mathrm{O}_{3} \mathrm{SiNa} 307.1706$, found 307.1690.

tert-butyldimethyl(((1R,3S,4R,5S,6R)-3-methyl-6-vinyl-2,7-dioxabicyclo[3.2.1]octan-4yl) oxy)silane (10a)

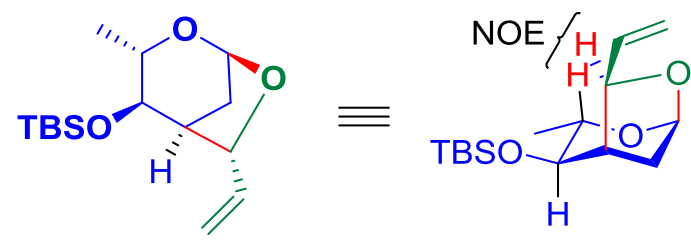

$10 a$

Yield: $28.5 \mathrm{mg}, 95 \%$. Rf: 0.6 (5\% EtOAc/hexane). 10a: IR (neat): 2953, 2929, 2896, 2857, $1730 \mathrm{~cm}^{-1}$. ${ }^{1} \mathrm{H}$ NMR $\left(500 \mathrm{MHz}, \mathrm{CDCl}_{3}\right): \delta 5.79$ (ddd, $1 \mathrm{H}, J=5.5 \mathrm{~Hz}, J=10.5 \mathrm{~Hz}, J=16.0 \mathrm{~Hz}$ ), $5.37(\mathrm{t}, 1 \mathrm{H}, J=2.5 \mathrm{~Hz}), 5.26(\mathrm{dt}, 1 \mathrm{H}, J=1.5 \mathrm{~Hz}, J=17.0 \mathrm{~Hz}), 5.12(\mathrm{dt}, 1 \mathrm{H}, J=1.5 \mathrm{~Hz}, J=$ $10.5 \mathrm{~Hz}), 4.83(\mathrm{~d}, 1 \mathrm{H}, J=5.0 \mathrm{~Hz}), 3.61-3.66(\mathrm{~m}, 1 \mathrm{H}), 3.36(\mathrm{dt}, 1 \mathrm{H}, J=2.5 \mathrm{~Hz}, J=8.0 \mathrm{~Hz})$, $2.25(\mathrm{t}, 1 \mathrm{H}, J=3.0 \mathrm{~Hz}), 1.87(\mathrm{dq}, 1 \mathrm{H}, J=2.5 \mathrm{~Hz}, J=12.0 \mathrm{~Hz}), 1.62(\mathrm{~d}, 1 \mathrm{H}, J=11.5 \mathrm{~Hz}), 1.22$ (d, $3 \mathrm{H}, J=6.5 \mathrm{~Hz}), 0.90(\mathrm{~s}, 9 \mathrm{H}), 0.08(\mathrm{~s}, 6 \mathrm{H}) .{ }^{13} \mathbf{C}$ NMR (125 MHz, $\left.\mathbf{C D C l}_{3}\right): \delta$ 137.6, 115.3, 98.7, 78.9, 73.8, 71.3, 45.4, 34.1, 25.6, 18.4, 17.8, -4.3, -4.8. HRMS (ESI-TOF) $\mathrm{m} / \mathrm{z}:[\mathrm{M}+\mathrm{Na}]^{+}$ calcd for $\mathrm{C}_{15} \mathrm{H}_{28} \mathrm{O}_{3} \mathrm{SiNa} 307.1706$, found 307.1691.

(((3R,6S)-6-(allyloxy)-3,6-dihydro-2H-pyran-3-yl)oxy)(tert-butyl)dimethylsilane (S11)<smiles>C=CCO[C@H]1C=C[C@@H](O[AsH3])CO1</smiles>

Yield: $169 \mathrm{mg}, 98 \% . \mathrm{R}_{\mathrm{f}}: 0.47$ (5\% EtOAc/hexane). S11: IR (neat): 2952, 2928, 2885, $2856 \mathrm{~cm}^{-}$ 1. ${ }^{1} \mathrm{H}$ NMR (500 MHz, CDCl $)$ : $\delta 5.96-6.00(\mathrm{~m}, 1 \mathrm{H}), 5.91-5.94(\mathrm{~m}, 1 \mathrm{H}), 5.85$ (ddd, $1 \mathrm{H}, J=0.5$ $\mathrm{Hz}, J=3.0 \mathrm{~Hz}, J=10.0 \mathrm{~Hz}$ ), $5.29(\mathrm{dq}, 1 \mathrm{H}, J=1.5 \mathrm{~Hz}, J=17.0 \mathrm{~Hz}), 5.19(\mathrm{dq}, 1 \mathrm{H}, J=1.5 \mathrm{~Hz}$, $J=10.5 \mathrm{~Hz}$ ), $5.04(\mathrm{~d}, 1 \mathrm{H}, J=2.5 \mathrm{~Hz}), 4.25$ (ddt, $1 \mathrm{H}, J=1.5 \mathrm{~Hz}, J=5.0 \mathrm{~Hz}, J=12.5 \mathrm{~Hz}$ ), 
4.04-4.10 (m, 2H), 3.98-4.00 (m, 1H), 3.68(dq, $1 \mathrm{H}, J=1.5 \mathrm{~Hz}, J=12.0 \mathrm{~Hz}), 0.91(\mathrm{~s}, 9 \mathrm{H}), 0.10$ (s, 6H). ${ }^{13} \mathrm{C}$ NMR (125 MHz, $\mathbf{C D C l}_{3}$ ): $\delta$ 134.3, 130.0, 127.7, 117.3, 92.9, 68.6, 65.3, 62.3, 25.8, 18.2, -4.5, -4.6. HRMS (ESI-TOF) $\mathrm{m} / \mathrm{z}$ : $[\mathrm{M}+\mathrm{Na}]^{+}$calcd for $\mathrm{C}_{14} \mathrm{H}_{26} \mathrm{O}_{3} \mathrm{SiNa} 293.1549$, found 293.1540 .

(R)-1-((3R,4R)-3-((tert-butyldimethylsilyl)oxy)-3,4-dihydro-2H-pyran-4-yl)prop-2-en-1-ol (11a)

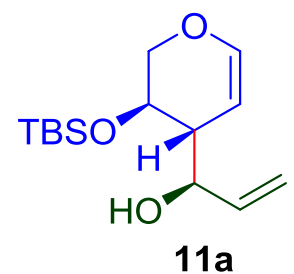

Yield: $44 \mathrm{mg}, 40 \%$. $\mathrm{R}_{\mathrm{f}}$ : 0.25 (5\% EtOAc/hexane). 11a: IR (neat): 3451, 2926, 2854, 1740, $1641 \mathrm{~cm}^{-1}$. ${ }^{1} \mathrm{H}$ NMR $\left(500 \mathrm{MHz}, \mathrm{CDCl}_{3}\right): \delta 6.47$ (dd, $\left.1 \mathrm{H}, J=2.5 \mathrm{~Hz}, J=6.0 \mathrm{~Hz}\right), 5.91$ (ddd, $1 \mathrm{H}$, $J=4.0 \mathrm{~Hz}, J=11.0 \mathrm{~Hz}, J=17.5 \mathrm{~Hz}), 5.36(\mathrm{dt}, 1 \mathrm{H}, J=1.5 \mathrm{~Hz}, J=17.0 \mathrm{~Hz}), 5.25(\mathrm{dt}, 1 \mathrm{H}, J=$ $1.5 \mathrm{~Hz}, J=10.5 \mathrm{~Hz}$ ), 4.50 (dd, $1 \mathrm{H}, J=2.5 \mathrm{~Hz}, J=6.0 \mathrm{~Hz}), 4.36-4.39(\mathrm{~m}, 1 \mathrm{H}), 4.06(\mathrm{ddd}, 1 \mathrm{H}$, $J=4.5 \mathrm{~Hz}, J=8.0 \mathrm{~Hz}, J=12.0 \mathrm{~Hz}), 4.00(\mathrm{dd}, 1 \mathrm{H}, J=4.0 \mathrm{~Hz}, J=10.0 \mathrm{~Hz}), 3.61(\mathrm{t}, 1 \mathrm{H}, J=$ $10.0 \mathrm{~Hz}), 2.36-2.39(\mathrm{~m}, 1 \mathrm{H}), 1.68(\mathrm{~d}, 1 \mathrm{H}, J=8.5 \mathrm{~Hz}), 0.92(\mathrm{~s}, 9 \mathrm{H}), 0.15(\mathrm{~s}, 3 \mathrm{H}), 0.13(\mathrm{~s}, 3 \mathrm{H})$. ${ }^{13} \mathrm{C}$ NMR (100 MHz, $\mathrm{CDCl}_{3}$ ): $\delta 146.4,139.4,114.8,96.8,70.4,69.0,65.4,46.1,25.7,17.9$, 4.3, -4.8. HRMS (ESI-TOF) $\mathrm{m} / \mathrm{z}$ : $[\mathrm{M}+\mathrm{Na}]^{+}$calcd for $\mathrm{C}_{14} \mathrm{H}_{26} \mathrm{O}_{3} \mathrm{SiNa} 293.1549$, found 293.1540 .

(S)-1-((3R,4R)-3-((tert-butyldimethylsilyl)oxy)-3,4-dihydro-2H-pyran-4-yl)prop-2-en-1-ol (11b)

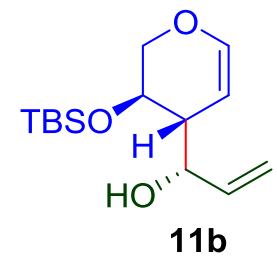

Yield: $44 \mathrm{mg}, 40 \%$. $\mathrm{R}_{\mathrm{f}}$ : 0.25 (5\% EtOAc/hexane). 11b: IR (neat): 3452, 3070, 2954, 2929, 2888, 2857, 1738, $1649 \mathrm{~cm}^{-1}$. ${ }^{1} \mathrm{H}$ NMR (500 MHz, $\left.\mathbf{C D C l}_{3}\right): \delta 6.39$ (dd, $1 \mathrm{H}, J=2.0 \mathrm{~Hz}, J=6.0$ $\mathrm{Hz}), 5.83-5.90(\mathrm{~m}, 1 \mathrm{H}), 5.30(\mathrm{dt}, 1 \mathrm{H}, J=1.5 \mathrm{~Hz}, J=17.5 \mathrm{~Hz}), 5.24(\mathrm{dt}, 1 \mathrm{H}, J=1.0 \mathrm{~Hz}, J=10.5$ $\mathrm{Hz}), 4.61(\mathrm{dd}, 1 \mathrm{H}, J=2.5 \mathrm{~Hz}, J=6.0 \mathrm{~Hz}), 4.13(\mathrm{t}, 1 \mathrm{H}, J=6.5 \mathrm{~Hz}), 3.90-3.96(\mathrm{~m}, 2 \mathrm{H}), 3.62-$ $3.67(\mathrm{~m}, 1 \mathrm{H}), 2.35-2.38(\mathrm{~m}, 2 \mathrm{H}), 0.91(\mathrm{~s}, 9 \mathrm{H}), 0.13(\mathrm{~s}, 3 \mathrm{H}), 0.12(\mathrm{~s}, 3 \mathrm{H}) .{ }^{13} \mathrm{C}$ NMR (125 MHz,

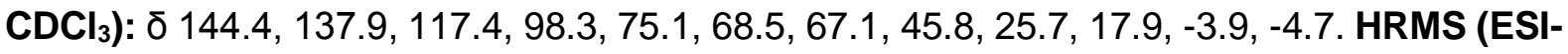
TOF) $\mathrm{m} / \mathrm{z}$ : [M+Na] ${ }^{+}$calcd for $\mathrm{C}_{14} \mathrm{H}_{26} \mathrm{O}_{3} \mathrm{SiNa} 293.1549$, found 293.1540.

(((3R,6S)-6-(benzyloxy)-3,6-dihydro-2H-pyran-3-yl)oxy)(tert-butyl)dimethylsilane (S12)<smiles>CC(C)(O)O[C@H]1C=C[C@@H](OCc2ccccc2)CO1</smiles>

S12

Yield: $152 \mathrm{mg}, 98 \% . \mathrm{R}_{\mathrm{f}}: 0.45$ (5\% EtOAc/hexane). S12: IR (neat): 2952, 2927, $2856 \mathrm{~cm}^{-1} .{ }^{1} \mathbf{H}$ NMR (400 MHz, CDCl $\left.)_{3}\right)$ ס 7.28-7.40 (m, 5H), 5.99-6.03 (m, 1H), 5.87-5.90 (m, 1H), $5.12(\mathrm{~d}$, $1 \mathrm{H}, J=2.8 \mathrm{~Hz}), 4.82(\mathrm{~d}, 1 \mathrm{H} J=11.6 \mathrm{~Hz}), 4.60(\mathrm{~d}, 1 \mathrm{H}, J=12.0 \mathrm{~Hz}), 4.15(\mathrm{dd}, 1 \mathrm{H}, J=3.2 \mathrm{~Hz}$, $J=8.0 \mathrm{~Hz}), 4.00-4.02(\mathrm{~m}, 1 \mathrm{H}), 3.72-3.76(\mathrm{~m}, 1 \mathrm{H}), 0.93(\mathrm{~s}, 9 \mathrm{H}), 0.13(\mathrm{~m}, 3 \mathrm{H}), 0.12(\mathrm{~m}, 3 \mathrm{H})$. 
${ }^{13} \mathrm{C}$ NMR (100 MHz, $\left.\mathrm{CDCl}_{3}\right): \delta 137.8,130.0,128.4,128.0,127.7,127.7,92.8,69.6,65.3,62.3$, 25.9, 18.3, -4.5, -4.6. HRMS (ESI-TOF) $\mathrm{m} / \mathrm{z}$ : $[\mathrm{M}+\mathrm{K}]^{+}$calcd for $\mathrm{C}_{18} \mathrm{H}_{28} \mathrm{O}_{3} \mathrm{SiK} 359.1445$, found 359.1439 .

(R)-((3R,4R)-3-((tert-butyldimethylsilyl)oxy)-3,4-dihydro-2H-pyran-4-yl)(phenyl)methan -ol (12a)<smiles>CC(C)(C)O[C@@H]1COC=C[C@@H]1[C@H](O)c1ccccc1</smiles>

$12 \mathbf{a}$

Yield: $43 \mathrm{mg}, 39 \%$. Rf: 0.25 (5\% EtOAc/hexane). 12a: IR (neat): 3455, 3063, 3029, 2953, 2927, 2855, 1738, $1648 \mathrm{~cm}^{-1}$. ${ }^{1} \mathrm{H}$ NMR (500 MHz, $\left.\mathbf{C D C l}_{3}\right): \delta$ 7.34-7.40 (m, 4H), 7.28-7.33 (m, $1 \mathrm{H}), 6.33(\mathrm{dd}, 1 \mathrm{H}, J=2.0 \mathrm{~Hz}, J=6.0 \mathrm{~Hz}), 4.54(\mathrm{dd}, 1 \mathrm{H}, J=1.5 \mathrm{~Hz}, J=8.5 \mathrm{~Hz}), 4.17(\mathrm{dd}, 1 \mathrm{H}$, $J=3.0 \mathrm{~Hz}, J=6.0 \mathrm{~Hz}$ ), 4.12-4.15 (m, 1H), $3.99(\mathrm{dd}, 1 \mathrm{H}, J=3.0 \mathrm{~Hz}, J=10.5 \mathrm{~Hz}), 3.74-3.77$ (m, 1H), $3.11(\mathrm{~d}, 1 \mathrm{H}, J=2.0 \mathrm{~Hz}), 2.51-2.54(\mathrm{~m}, 1 \mathrm{H}), 0.94(\mathrm{~s}, 9 \mathrm{H}), 0.16(\mathrm{~s}, 3 \mathrm{H}), 0.14(\mathrm{~s}, 3 \mathrm{H}) .{ }^{13} \mathrm{C}$ NMR (125 MHz, $\mathrm{CDCl}_{3}$ ): $\delta$ 144.4, 141.7, 128.3, 127.8, 127.0, 98.4, 78.0, 68.5, 68.2, 46.7, 25.7, 17.9, -4.0, -4.8. HRMS (ESI-TOF) $\mathrm{m} / \mathrm{z}$ : $[\mathrm{M}+\mathrm{Na}]^{+}$calcd for $\mathrm{C}_{18} \mathrm{H}_{28} \mathrm{O}_{3} \mathrm{SiNa} 343.1706$, found 343.1705.

(S)-((3R,4R)-3-((tert-butyldimethylsilyl)oxy)-3,4-dihydro-2H-pyran-4-yl)(phenyl) methanol (12b)

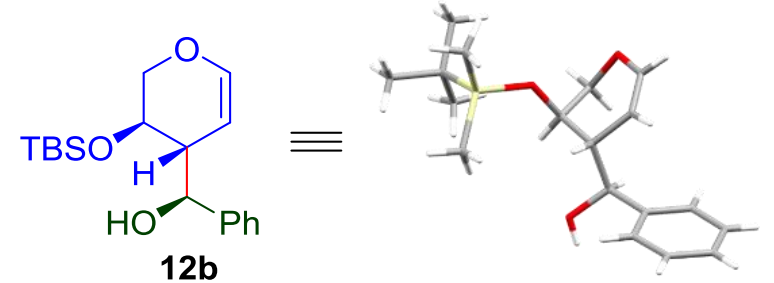

Yield: 43 mg, 39\%, $\mathrm{R}_{\mathrm{f}}: 0.24$ (5\% EtOAc/hexane). 12b: IR (neat): 3467, 2952, 2927, 2855, 1737, $1647 \mathrm{~cm}^{-1} .{ }^{1} \mathbf{H}$ NMR (500 MHz, $\left.\mathbf{C D C l}_{3}\right): \delta$ 7.35-7.41 (m, 4H), 7.28-7.31 (m, 1H), 6.46 (dd, $1 \mathrm{H}, J=2.0 \mathrm{~Hz}, J=6.0 \mathrm{~Hz}$ ), $5.02(\mathrm{dd}, 1 \mathrm{H}, J=2.5 \mathrm{~Hz}, J=7.0 \mathrm{~Hz}), 4.40$ (dd, $1 \mathrm{H}, J=2.5$ $\mathrm{Hz}, J=6.0 \mathrm{~Hz}$ ), 4.11-4.16 (m, 1H), 4.02 (dd, $1 \mathrm{H}, J=4.0 \mathrm{~Hz}, J=10.5 \mathrm{~Hz}$ ), $3.63(\mathrm{t}, 1 \mathrm{H}, J=10.0$ $\mathrm{Hz}), 2.55-2.58(\mathrm{~m}, 1 \mathrm{H}), 1.96(\mathrm{~d}, 1 \mathrm{H}, J=7.0 \mathrm{~Hz}), 0.96(\mathrm{~s}, 9 \mathrm{H}), 0.18(\mathrm{~s}, 3 \mathrm{H}), 0.15(\mathrm{~s}, 3 \mathrm{H}) .{ }^{13} \mathrm{C}$ NMR (125 MHz, $\left.\mathrm{CDCl}_{3}\right): \delta$ 146.6, 142.9, 128.3, 127.1, 125.4, 95.7, 71.2, 68.9, 65.4, 48.5, 25.8, 17.9, -4.3, -4.9. HRMS (ESI-TOF) $\mathrm{m} / z$ : $[\mathrm{M}+\mathrm{Na}]^{+}$calcd for $\mathrm{C}_{18} \mathrm{H}_{28} \mathrm{O}_{3} \mathrm{SiNa} 343.1706$, found 343.1701 .

(R)-1-((2R,3S,4S)-3-((tert-butyldimethylsilyl)oxy)-2-(((tert-butyldimethylsilyl)oxy) methyl) -3,4-dihydro-2H-pyran-4-yl)prop-2-en-1-ol (14a)

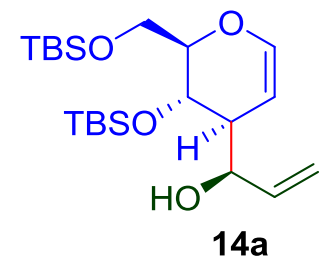

Yield: $34 \mathrm{mg}, 31 \%$. Rf: 0.45 (5\% EtOAc/hexane). 14a: IR (neat): 3068, 2953, 2928, 2885, 2856, 2359, 2339, 1736, $1646 \mathrm{~cm}^{-1}$. ${ }^{1} \mathrm{H}$ NMR (400 MHz, $\left.\mathbf{C D C l}_{3}\right): \delta 6.42$ (dd, $1 \mathrm{H}, J=2.0 \mathrm{~Hz}, J$ 
$=6.0 \mathrm{~Hz}$ ), 5.88 (ddd, $1 \mathrm{H}, J=6.8 \mathrm{~Hz}, J=10.4 \mathrm{~Hz}, J=17.2 \mathrm{~Hz}), 5.34(\mathrm{dt}, 1 \mathrm{H}, J=1.2 \mathrm{~Hz}, J=$ $17.2 \mathrm{~Hz}$ ), 5.27 (dt, $1 \mathrm{H}, J=1.2 \mathrm{~Hz}, J=10.2 \mathrm{~Hz}$ ), 4.73 (dd, $1 \mathrm{H}, J=2.4 \mathrm{~Hz}, J=6.0 \mathrm{~Hz}$ ), $4.42-$ $4.45(\mathrm{~m}, 1 \mathrm{H}), 3.84-3.92(\mathrm{~m}, 2 \mathrm{H}), 3.79(\mathrm{t}, 1 \mathrm{H}, J=8.0 \mathrm{~Hz}), 3.59(\mathrm{dt}, 1 \mathrm{H}, J=3.2 \mathrm{~Hz}, J=8.4 \mathrm{~Hz})$, 2.52-2.56 (m, 1H), $1.66(\mathrm{bs}, 1 \mathrm{H}), 0.93(\mathrm{~s}, 9 \mathrm{H}), 0.91(\mathrm{~s}, 9 \mathrm{H}), 0.16(\mathrm{~s}, 3 \mathrm{H}), 0.14(\mathrm{~s}, 3 \mathrm{H}), 0.09(\mathrm{~s}$, $3 \mathrm{H}), 0.07$ (s, 3H). ${ }^{13} \mathrm{C}$ NMR (125 MHz, $\mathbf{C D C l}_{3}$ ): $\delta 143.9,137.3,117.7,98.5,79.2,72.4,65.7$, 61.9, 46.9, 26.0, 25.9, 18.4, 18.3, -4.0, -4.0, -4.9, -5.3. HRMS (ESI-TOF) $\mathrm{m} / \mathrm{z}$ : [M+Na] ${ }^{+}$calcd for $\mathrm{C}_{21} \mathrm{H}_{42} \mathrm{O}_{4} \mathrm{Si}_{2} \mathrm{Na}$ 437.2519, found 437.2515.

(S)-1-((2R,3S,4S)-3-((tert-butyldimethylsilyl)oxy)-2-(((tert-butyldimethylsilyl)oxy) methyl)-3,4-dihydro-2H-pyran-4-yl)prop-2-en-1-ol (14b)

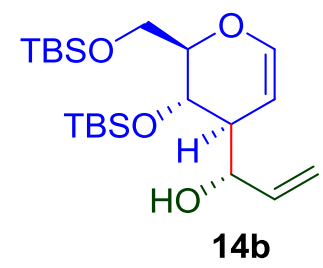

Yield: 34 mg, 31\%. Rf: 0.4 (5\% EtOAc/hexane). 14b: IR (neat): 3068, 2953, 2928, 2885, 2856, 2359, 2339, 1736, $1646 \mathrm{~cm}^{-1} .{ }^{1} \mathrm{H}$ NMR (500 MHz, $\left.\mathbf{C D C l}_{3}\right): \delta 6.53$ (dd, $1 \mathrm{H}, J=2.0 \mathrm{~Hz}, J=6.0$ $\mathrm{Hz}$ ), 5.89 (ddd, $1 \mathrm{H}, J=4.0 \mathrm{~Hz}, J=11.0 \mathrm{~Hz}, J=17.5 \mathrm{~Hz}$ ), 5.33 (dt, $1 \mathrm{H}, J=1.5 \mathrm{~Hz}, J=17.5$ $\mathrm{Hz}), 5.24(\mathrm{dt}, 1 \mathrm{H}, J=1.5 \mathrm{~Hz}, J=11.0 \mathrm{~Hz}), 4.45-4.47(\mathrm{~m}, 2 \mathrm{H}), 4.06(\mathrm{t}, 1 \mathrm{H}, J=3.5 \mathrm{~Hz}), 3.86-$ $3.94(\mathrm{~m}, 2 \mathrm{H}), 3.59-3.62(\mathrm{~m}, 1 \mathrm{H}), 2.35(\mathrm{dq}, 1 \mathrm{H}, J=2.0 \mathrm{~Hz}, J=8.0 \mathrm{~Hz}), 1.41(\mathrm{~d}, 1 \mathrm{H}, J=8.5 \mathrm{~Hz})$, $0.92(\mathrm{~s}, 9 \mathrm{H}), 0.91(\mathrm{~s}, 9 \mathrm{H}), 0.18(\mathrm{~s}, 3 \mathrm{H}), 0.17(\mathrm{~s}, 3 \mathrm{H}), 0.09(\mathrm{~s}, 3 \mathrm{H}), 0.08(\mathrm{~s}, 3 \mathrm{H}) .{ }^{13} \mathrm{C}$ NMR (125 $\left.\mathbf{M H z}, \mathrm{CDCl}_{3}\right): \delta 147.0,139.9,114.5,95.3,79.8,68.7,64.9,62.3,46.9,26.0,25.9,18.5,18.3$, $-4.2,-4.9,-4.9,-5.3$. HRMS (ESI-TOF) $\mathrm{m} / \mathrm{z}$ : [M+Na] ${ }^{+}$calcd for $\mathrm{C}_{21} \mathrm{H}_{42} \mathrm{O}_{4} \mathrm{Si}_{2} \mathrm{Na} 437.2519$, found 437.2517 .

(((2R,3S,6R)-6-(benzyloxy)-2-(((tert-butyldimethylsilyl)oxy)methyl)-3,6-dihydro-2Hpyran-3-yl)oxy)(tert-butyl)dimethylsilane (S15)

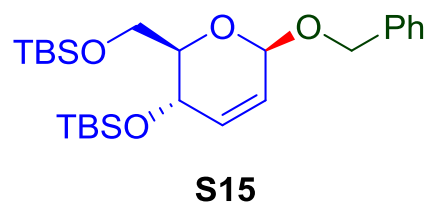

Yield: 192 mg, 98\%. $R_{\mathrm{f}}$ : 0.55(5\% EtOAc/hexane). S15: IR (neat): 2957, 2929, 2881, $2856 \mathrm{~cm}^{-}$ 1. ${ }^{1} \mathrm{H}$ NMR (400 MHz, $\left.\mathbf{C D C l}_{3}\right): \delta 7.29-7.41(\mathrm{~m}, 5 \mathrm{H}), 5.93(\mathrm{dq}, 1 \mathrm{H}, J=1.6 \mathrm{~Hz}, J=10.0 \mathrm{~Hz}), 5.78$ $(\mathrm{dt}, 1 \mathrm{H}, J=1.6 \mathrm{~Hz}, J=10 \mathrm{~Hz}), 5.24-5.26(\mathrm{~m}, 1 \mathrm{H}), 4.88(\mathrm{~d}, 1 \mathrm{H}, J=11.6 \mathrm{~Hz}), 4.64(\mathrm{~d}, 1 \mathrm{H}, J=$ $11.6 \mathrm{~Hz}), 4.31-4.34(\mathrm{~m}, 1 \mathrm{H}), 3.82-3.92(\mathrm{~m}, 2 \mathrm{H}), 3.61-3.65(\mathrm{~m}, 1 \mathrm{H}), 0.96(\mathrm{~s}, 9 \mathrm{H}), 0.94(\mathrm{~s}, 9 \mathrm{H})$, 0.14-0.15 (m, 12H). ${ }^{13} \mathrm{C}$ NMR (100 MHz, $\left.\mathbf{C D C l}_{3}\right): \delta 137.7,132.9,128.3,128.1,127.6,127.5$, 95.4, 79.3, 69.0, 63.2, 62.8, 25.9, 25.8, 18.4, 18.0, -4.4, -4.8, -5.2, -5.3. HRMS (ESI-TOF) $\mathrm{m} / \mathrm{z}$ : $[\mathrm{M}+\mathrm{Na}]^{+}$calcd for $\mathrm{C}_{25} \mathrm{H}_{44} \mathrm{O}_{4} \mathrm{Si}_{2} \mathrm{Na} 487.2676$, found 487.2672.

(S)-((2R,3S,4S)-3-((tert-butyldimethylsilyl)oxy)-2-(((tert-butyldimethylsilyl)oxy) methyl)3,4-dihydro-2H-pyran-4-yl)(phenyl)methanol (15a)

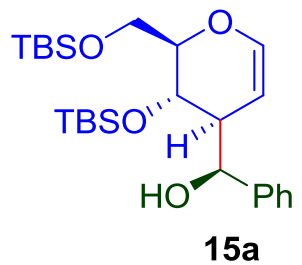


Yield: 56 mg, 51\%. Rf: 0.4 (5\% EtOAc/hexane). 15a: IR (neat): 3532, 2953, 2927, 2855, 1736 $\mathrm{cm}^{-1} .{ }^{1} \mathrm{H}$ NMR $\left(400 \mathrm{MHz}, \mathrm{CDCl}_{3}\right): \delta 7.29-7.39(\mathrm{~m}, 5 \mathrm{H}), 6.28(\mathrm{dd}, 1 \mathrm{H}, J=2.0 \mathrm{~Hz}, J=6.4 \mathrm{~Hz})$, $4.69(\mathrm{dd}, 1 \mathrm{H}, J=2.4 \mathrm{~Hz}, J=7.6 \mathrm{~Hz}), 4.36(\mathrm{dd}, 1 \mathrm{H}, J=3.6 \mathrm{~Hz}, J=6.4 \mathrm{~Hz}), 4.25-4.28(\mathrm{~m}, 1 \mathrm{H})$, 3.83-3.89 (m, 3H), 2.55-2.60 (m, 1H), $2.42(\mathrm{~d}, 1 \mathrm{H}, J=2.4 \mathrm{~Hz}), 0.95(\mathrm{~s}, 9 \mathrm{H}), 0.91(\mathrm{~s}, 9 \mathrm{H}), 0.21$ (s, 3H), 0.20 (s, 3H), 0.09 (s, 3H), 0.05 (s, 3H). $\left.{ }^{13} \mathbf{C ~ N M R ~ ( 1 0 0 ~ M H z , ~} \mathbf{C D C l}_{3}\right): \delta$ 143.0, 142.1, 128.2, 127.8, 127.0, 98.8, 79.0, 75.8, 66.8, 61.7, 47.2, 26.0, 25.9, 18.3, 18.3, -3.8, -3.9, -5.3, -5.3. HRMS (ESI-TOF) $\mathrm{m} / \mathrm{z}$ : [M+Na] ${ }^{+}$calcd for $\mathrm{C}_{25} \mathrm{H}_{44} \mathrm{O}_{4} \mathrm{Si}_{2} \mathrm{Na} 487.2676$, found 487.2674.

(R)-((2R,3S,4S)-3-((tert-butyldimethylsilyl)oxy)-2-(((tert-butyldimethylsilyl)oxy) methyl)3,4-dihydro-2H-pyran-4-yl)(phenyl)methanol (15b)

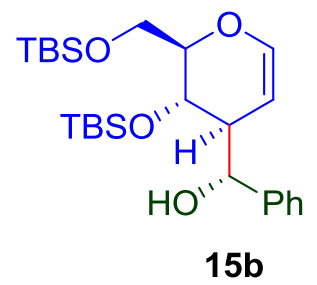

Yield: $35 \mathrm{mg}, 32 \%$. Rf: 0.4 (5\% Acetone/hexane). 15b: IR (neat): 3531, 2926, 2855, 2020, 1732, $1652 \mathrm{~cm}^{-1} .{ }^{1} \mathbf{H}$ NMR (400 MHz, $\left.\mathbf{C D C l}_{3}\right): \delta 7.35-7.41(\mathrm{~m}, 4 \mathrm{H}), 7.27-7.31(\mathrm{~m}, 1 \mathrm{H}), 6.50$ $(\mathrm{dd}, 1 \mathrm{H}, J=2.4 \mathrm{~Hz}, J=6.0 \mathrm{~Hz}$ ), $5.14(\mathrm{dd}, 1 \mathrm{H}, J=1.6 \mathrm{~Hz}, J=8.0 \mathrm{~Hz}), 4.26(\mathrm{dd}, 1 \mathrm{H}, J=2.0$ $\mathrm{Hz}, J=6.0 \mathrm{~Hz}), 4.18-4.22(\mathrm{~m}, 1 \mathrm{H}), 3.91-3.98(\mathrm{~m}, 2 \mathrm{H}), 3.61-3.65(\mathrm{~m}, 1 \mathrm{H}), 2.63(\mathrm{dq}, 1 \mathrm{H}, J=2.4$ $\mathrm{Hz}, J=8.4 \mathrm{~Hz}), 1.85(\mathrm{~d}, 1 \mathrm{H}, J=8.0 \mathrm{~Hz}), 1.0(\mathrm{~s}, 9 \mathrm{H}), 0.95(\mathrm{~s}, 9 \mathrm{H}), 0.26(\mathrm{~s}, 3 \mathrm{H}), 0.24(\mathrm{~s}, 3 \mathrm{H})$, 0.12 (s, 6H). ${ }^{13} \mathbf{C}$ NMR (100 MHz, $\left.\mathbf{C D C l}_{3}\right): \delta$ 147.1, 143.2, 128.3, 126.9, 125.2, 95.1, 79.9, 69.7, 65.2, 49.4, 26.1, 25.9, 18.5, 18.3, -4.1, -4.7, -4.9, -5.3. HRMS (ESI-TOF) $\mathrm{m} / \mathrm{z}:[\mathrm{M}+\mathrm{Na}]^{+}$ calcd for $\mathrm{C}_{25} \mathrm{H}_{44} \mathrm{O}_{4} \mathrm{Si}_{2} \mathrm{Na} 487.2676$, found 487.2671 .

(2R,3R,6R)-6-(allyloxy)-2-((trityloxy)methyl)-3,6-dihydro-2H-pyran-3-ol (SS16)

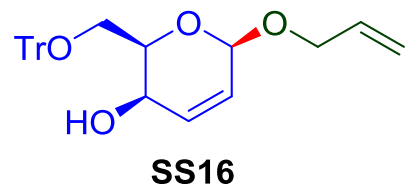

Yield: $170 \mathrm{mg}, 85 \%$. Rf: 0.25 (20\% EtOAc/hexane). SS16: IR (neat): 3430, 3056, 3020, 2926, 2871, 2359, 2342, 1961, 1711, 1644, $1596 \mathrm{~cm}^{-1}$. ${ }^{1} \mathbf{H}$ NMR (500 MHz, $\left.\mathbf{C D C l}_{3}\right): \delta$ 7.48-7.52 (m, $6 \mathrm{H}), 7.24-7.34(\mathrm{~m}, 9 \mathrm{H}), 6.15(\mathrm{ddd}, 1 \mathrm{H}, J=1.5 \mathrm{~Hz}, J=5.0 \mathrm{~Hz}, J=10.0 \mathrm{~Hz}), 5.94-6.02(\mathrm{~m}, 1 \mathrm{H})$, $5.88(\mathrm{~d}, 1 \mathrm{H}, J=10.0 \mathrm{~Hz}), 5.33-5.37(\mathrm{~m}, 1 \mathrm{H}), 5.23-5.25(\mathrm{~m}, 1 \mathrm{H}), 5.17(\mathrm{~d}, 1 \mathrm{H}, J=1.0 \mathrm{~Hz}), 4.43$ (ddt, $1 \mathrm{H}, J=1.0 \mathrm{~Hz}, J=5.0 \mathrm{~Hz}, J=12.5 \mathrm{~Hz}$ ), 4.21 (dd, $1 \mathrm{H}, J=6.5 \mathrm{~Hz}, J=13.0 \mathrm{~Hz}$ ), 3.95 (bs, $1 \mathrm{H}), 3.83(\mathrm{td}, 1 \mathrm{H}, J=2.0 \mathrm{~Hz}, J=6.5 \mathrm{~Hz}), 3.51(\mathrm{dd}, 1 \mathrm{H}, J=6.5 \mathrm{~Hz}, J=10.0 \mathrm{~Hz}), 3.31(\mathrm{dd}, 1 \mathrm{H}$, $J=5.5 \mathrm{~Hz}, J=10.0 \mathrm{~Hz}), 1.87(\mathrm{~d}, 1 \mathrm{H}, J=5.5 \mathrm{~Hz}) .{ }^{13} \mathbf{C ~ N M R}\left(125 \mathbf{M H z}, \mathbf{C D C l}_{3}\right): \delta 143.9,134.1$, 130.9, 130.9, 128.7, 127.8, 127.0, 117.6, 97.1, 86.7, 74.9, 69.1, 63.3, 62.9. HRMS (ESI-TOF) $\mathrm{m} / \mathrm{z}:[\mathrm{M}+\mathrm{Na}]^{+}$calcd for $\mathrm{C}_{28} \mathrm{H}_{28} \mathrm{O}_{4} \mathrm{Na} 451.1886$, found 451.1883 .

(((2R,3R,6R)-6-(allyloxy)-2-((trityloxy)methyl)-3,6-dihydro-2H-pyran-3-yl)oxy) (tert-butyl )dimethylsilane (S16)

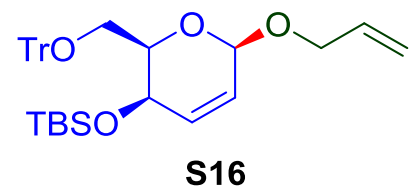


Yield: $180 \mathrm{mg}, 98 \%$. Rf: 0.75 (20\% EtOAc/hexane). S16: IR (neat): 3091, 3057, 2953, 2927, 2876, 2854, $1596 \mathrm{~cm}^{-1}$. ${ }^{1} \mathrm{H}$ NMR (400 MHz, $\left.\mathbf{C D C l}_{3}\right): \delta$ 7.53-7.55 (m, 6H), 7.32-7.35 (m, 6H), 7.25-7.29 (m, 3H), 5.99-6.09 (m, 1H), 5.93-5.97 (m, 1H), $5.86(\mathrm{~d}, 1 \mathrm{H}, J=10.0 \mathrm{~Hz}), 5.36-5.40$ $(\mathrm{m}, 1 \mathrm{H}), 5.26(\mathrm{dd}, 1 \mathrm{H}, J=1.6 \mathrm{~Hz}, J=10.4 \mathrm{~Hz}), 5.22(\mathrm{~s}, 1 \mathrm{H}), 4.47-4.51(\mathrm{~m}, 1 \mathrm{H}), 4.29-4.34(\mathrm{~m}$, $1 \mathrm{H}), 3.97(\mathrm{bs}, 1 \mathrm{H}), 3.86-3.89(\mathrm{~m}, 1 \mathrm{H}), 3.59-3.64(\mathrm{~m}, 1 \mathrm{H}), 3.13(\mathrm{dd}, 1 \mathrm{H}, J=3.6 \mathrm{~Hz}, J=6.0 \mathrm{~Hz})$, $0.76(\mathrm{~s}, 9 \mathrm{H}),-0.01(\mathrm{~s}, 3 \mathrm{H}),-0.12(\mathrm{~s}, 3 \mathrm{H}) .{ }^{13} \mathbf{C} \mathbf{~ N M R}\left(100 \mathbf{~ M H z}, \mathbf{C D C l}_{3}\right): \delta 144.2,134.5,130.9$, 129.9, 128.7, 127.7, 126.8, 117.4, 96.2, 86.6, 75.4, 68.5, 64.5, 63.6, 25.7, 17.9, -4.3, -4.9. HRMS (ESI-TOF) $\mathrm{m} / \mathrm{z}$ : [M+Na] ${ }^{+}$calcd for $\mathrm{C}_{34} \mathrm{H}_{42} \mathrm{O}_{4} \mathrm{SiNa} 565.2751$, found 565.2750.

(R)-1-((2R,3R,4S)-3-((tert-butyldimethylsilyl)oxy)-2-((trityloxy)methyl)-3,4-di hydro-2Hpyran-4-yl)prop-2-en-1-ol (16)

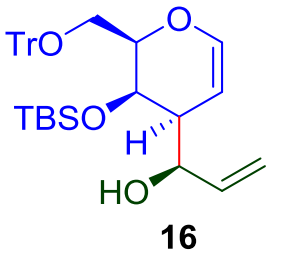

Yield: 90 mg, 90\%. Rf: 0.65 (20\% EtOAc/hexane). 16: IR (neat): 3554, 3060, 3023, 2950, 2928, 2886, 2855, 2359, 2339, 1646, $1596 \mathrm{~cm}^{-1}$. ${ }^{1} \mathbf{H}$ NMR (500 MHz, $\left.\mathbf{C D C l}_{3}\right): \delta$ 7.48-7.50 (m, $6 \mathrm{H}), 7.31-7.34(\mathrm{~m}, 6 \mathrm{H}), 7.25-7.28(\mathrm{~m}, 3 \mathrm{H}), 6.62(\mathrm{dd}, 1 \mathrm{H}, J=2.0 \mathrm{~Hz}, J=6.0 \mathrm{~Hz}$ ), 5.87 (ddd, $1 \mathrm{H}, J=4.0 \mathrm{~Hz}, J=11.0 \mathrm{~Hz}, J=17.0 \mathrm{~Hz}), 5.39(\mathrm{dt}, 1 \mathrm{H}, J=1.5 \mathrm{~Hz}, J=17.0 \mathrm{~Hz}), 5.22(\mathrm{dt}, 1 \mathrm{H}$, $J=1.5 \mathrm{~Hz}, J=10.5 \mathrm{~Hz}), 4.67(\mathrm{dt}, 1 \mathrm{H}, J=1.5 \mathrm{~Hz}, J=6.0 \mathrm{~Hz}), 4.38-4.39(\mathrm{~m}, 1 \mathrm{H}), 4.11(\mathrm{~d}, 1 \mathrm{H}$, $J=4.5 \mathrm{~Hz}$ ), 3.99 (dd, $1 \mathrm{H}, J=4.5 \mathrm{~Hz}, J=7.5 \mathrm{~Hz}$ ), $3.53(\mathrm{dd}, 1 \mathrm{H}, J=7.5 \mathrm{~Hz}, J=10.0 \mathrm{~Hz}$ ), 3.06 (dd, $1 \mathrm{H}, J=4.5 \mathrm{~Hz}, J=10.0 \mathrm{~Hz}), 2.75(\mathrm{~s}, 1 \mathrm{H}), 2.53-2.54(\mathrm{~m}, 1 \mathrm{H}), 0.77(\mathrm{~s}, 9 \mathrm{H}), 0.12(\mathrm{~s}, 3 \mathrm{H}),-$ $0.21(\mathrm{~s}, 3 \mathrm{H}) .{ }^{13} \mathrm{C}$ NMR (125 MHz, $\left.\mathbf{C D C l}_{3}\right): \delta$ 145.6, 143.8, 138.6, 128.7, 127.8, 127.0, 114.9, 96.2, 86.9, 77.6, 71.2, 68.8, 64.5, 42.7, 25.7, 18.1, -3.9, -4.4. HRMS (ESI-TOF) $\mathrm{m} / \mathrm{z}$ : [M+Na] ${ }^{+}$ calcd for $\mathrm{C}_{34} \mathrm{H}_{42} \mathrm{O}_{4} \mathrm{SiNa} 565.2751$, found 565.2750.

(((2R,3R,6R)-6-(benzyloxy)-2-((trityloxy)methyl)-3,6-dihydro-2H-pyran-3-yl)oxy)(tertbutyl)dimethylsilane (S17)

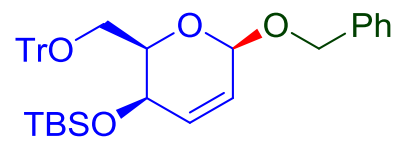

S17

Yield: 168 mg, 97\%. $R_{\mathrm{f}}$ : 0.75 (20\% EtOAc/hexane). S17: IR (neat): 3084, 3058, 3030, 2951, 2927, 2883, 2855, 2359, 2339, 1738, $1596 \mathrm{~cm}^{-1}$. ${ }^{1} \mathbf{H}$ NMR (400 MHz, $\left.\mathbf{C D C l}_{3}\right): \delta$ 7.54-7.56 (m, $6 \mathrm{H}), 7.31-7.337 .47(\mathrm{~m}, 11 \mathrm{H}), 7.24-7.29(\mathrm{~m}, 3 \mathrm{H}), 5.94(\mathrm{ddd}, 1 \mathrm{H}, J=1.2 \mathrm{~Hz}, J=4.4 \mathrm{~Hz}, J=$ $10.0 \mathrm{~Hz}), 5.87(\mathrm{~d}, 1 \mathrm{H}, J=10.4 \mathrm{~Hz}), 5.20-5.21(\mathrm{~m}, 1 \mathrm{H}), 4.99(\mathrm{~d}, J=12.0 \mathrm{~Hz}), 4.87(\mathrm{~d}, 1 \mathrm{H}, J=$ $12.0 \mathrm{~Hz}), 3.96-3.98(\mathrm{~m}, 1 \mathrm{H}), 3.85-3.89(\mathrm{~m}, 1 \mathrm{H}), 3.64(\mathrm{dd}, 1 \mathrm{H}, J=8.0 \mathrm{~Hz}, J=10.4 \mathrm{~Hz}), 3.13$ $(\mathrm{dd}, 1 \mathrm{H}, J=3.2 \mathrm{~Hz}, J=10.0 \mathrm{~Hz}), 0.76(\mathrm{~s}, 9 \mathrm{H}),-0.01(\mathrm{~s}, 3 \mathrm{H}),-0.12(\mathrm{~s}, 3 \mathrm{H}) .{ }^{13} \mathrm{C} \mathrm{NMR}(100 \mathrm{MHz}$, $\left.\mathrm{CDCl}_{3}\right): \delta 144.2,137.7,130.9,130.0,128.7,128.3,128.3,127.7,127.6,126.9,95.6,86.6$, 75.5, 68.9, 64.6, 63.7, 25.7, 18.0, -4.2, -4.9. HRMS (ESI-TOF) $\mathrm{m} / \mathrm{z}$ : $[\mathrm{M}+\mathrm{Na}]^{+}$calcd for $\mathrm{C}_{38} \mathrm{H}_{44} \mathrm{O}_{4} \mathrm{SiNa}$ 615.2907, found 615.2908. 

pyran-4-yl)(phenyl)methanol (17)

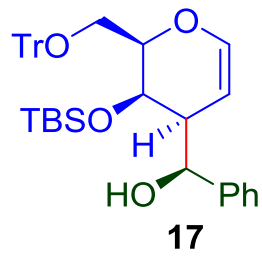

Yield: 88 mg, 80\%. Rf: 0.7 (20\% EtOAc/hexane). 17: IR (neat): 3544, 3059, 3027, 2953, 2929, 2886, 2855, 2359, 2332, 1650, $1598 \mathrm{~cm}^{-1}$. ${ }^{1} \mathrm{H}$ NMR (500 MHz, $\left.\mathbf{C D C l}_{3}\right): \delta$ 7.48-7.50 (m, 6H), 7.25-7.41 (m, 14H), 6.61 (dd, $1 \mathrm{H}, J=2.5 \mathrm{~Hz}, J=6.5 \mathrm{~Hz}$ ), $5.01(\mathrm{~s}, 1 \mathrm{H}), 4.56$ (dt, $1 \mathrm{H}, J=1.5$ $\mathrm{Hz}, J=6.0 \mathrm{~Hz}$ ), $4.25(\mathrm{~d}, 1 \mathrm{H}, J=4.0 \mathrm{~Hz}), 3.98(\mathrm{dd}, 1 \mathrm{H}, J=4.5 \mathrm{~Hz}, J=8.0 \mathrm{~Hz}), 3.55(\mathrm{dd}, 1 \mathrm{H}, J$ $=8.0 \mathrm{~Hz}, J=10.0 \mathrm{~Hz}), 3.18(\mathrm{~s}, 1 \mathrm{H}), 3.08(\mathrm{dd}, 1 \mathrm{H}, J=4.0 \mathrm{~Hz}, J=10.0 \mathrm{~Hz}), 2.75-2.76(\mathrm{~m}, 1 \mathrm{H})$, 0.80 (s, 9H), 0.17(s, 3H), -0.17 (s, 3H). $\left.{ }^{13} \mathbf{C ~ N M R ~ ( 1 2 5 ~ M H z , ~} \mathbf{C D C l}_{3}\right): \delta$ 145.9, 143.8, 142.7, 128.7, 128.2, 127.9, 127.8, 127.0, 125.7, 95.7, 87.0, 77.7, 72.7, 69.5, 64.5, 45.0, 25.8, 18.1, 3.9, -4.3. HRMS (ESI-TOF) $\mathrm{m} / \mathrm{z}$ : $[\mathrm{M}+\mathrm{Na}]^{+}$calcd for $\mathrm{C}_{38} \mathrm{H}_{44} \mathrm{O}_{4} \mathrm{SiNa} 615.2907$, found 615.2908.

(2R,4a $R, 6 R, 8 \mathrm{a} S)-6-($ allyloxy)-2-(4-methoxyphenyl)-4,4a,6,8a-tetrahydropyrano[3,2-d] $[1,3]$ dioxine (S18)<smiles>C=CCOC1C=CC2[CH-]C([15NH2])OC[C@H]2O1</smiles>

Yield: $160 \mathrm{mg}, 62 \%, \mathrm{R}_{\mathrm{f}}: 0.6$ (20\% EtOAc/hexane). S18: IR (neat): 3006, 2954, 2872, 1615, 1589, $1515 \mathrm{~cm}^{-1} .{ }^{1} \mathrm{H}$ NMR (500 MHz, $\left.\mathbf{C D C l}_{3}\right): \delta 7.44(\mathrm{~d}, 1 \mathrm{H}, J=8.5 \mathrm{~Hz}), 6.91(\mathrm{~d}, 1 \mathrm{H}, J=8.5$ $\mathrm{Hz}), 6.16(\mathrm{~d}, 1 \mathrm{H}, J=10.5 \mathrm{~Hz}), 5.92-6.00(\mathrm{~m}, 1 \mathrm{H}), 5.72(\mathrm{~d}, 1 \mathrm{H}, J=10.5 \mathrm{~Hz}), 5.58(\mathrm{~s}, 1 \mathrm{H}), 5.40$ (s, 1H), 5.33 (dd, $1 \mathrm{H}, J=1.5 \mathrm{~Hz}, J=17.0 \mathrm{~Hz}$ ), 5.23 (d, $1 \mathrm{H}, J=10.5 \mathrm{~Hz}$ ), 3.81 (s, 3H), 3.74$3.79(\mathrm{~m}, 1 \mathrm{H}) .{ }^{13} \mathrm{C}$ NMR (125 MHz, $\left.\mathbf{C D C l}_{3}\right): \delta$ 160.2, 134.0, 131.5, 129.9, 128.2, 127.5, 117.5, 113.7, 102.0, 97.9, 74.9, 70.5, 69.0, 68.7, 55.3. HRMS (ESI-TOF) $\mathrm{m} / \mathrm{z}$ : $[\mathrm{M}+\mathrm{Na}]^{+}$calcd for $\mathrm{C}_{17} \mathrm{H}_{20} \mathrm{O}_{5} \mathrm{Na} 327.1209$, found 327.1204.

(R/S)1-((2R,4aR,8S,8aS)-2-(4-methoxyphenyl)-4,4a,8,8a-tetrahydropyrano[3,2d][1,3]dioxin-8-yl)prop-2-en-1-ol (18a\&18b)<smiles>C=C[C@@H](O)[C@H]1C=CO[C@@H]2CO[C@@H](P)O[C@H]21</smiles>

18a:18b (2.3:1)

Yield: $58 \mathrm{mg}, 58 \%$. Rf: 0.5 (30\% EtOAc/hexane). 18a and 18b: IR (neat): 3503, 3091, 3072, 3005, 2968, 2897, 2866, 1686, 1637, 1613, 1588, $1516 \mathrm{~cm}^{-1}$. 18a : ${ }^{1} \mathbf{H}$ NMR (400 MHz, $\left.\mathbf{C D C l}_{3}\right)$ : $\delta 7.42(\mathrm{~d}, 2 \mathrm{H}, J=8.4 \mathrm{~Hz}), 6.91$ (d, $2 \mathrm{H}, J=8.8 \mathrm{~Hz}), 6.39(\mathrm{dd}, 1 \mathrm{H}, J=2.4 \mathrm{~Hz}, J=6.0 \mathrm{~Hz}), 5.88$ (ddd, $1 \mathrm{H}, J=7.2 \mathrm{~Hz}, J=10.0 \mathrm{~Hz}, J=17.2 \mathrm{~Hz}$ ), $5.60(\mathrm{~s}, 1 \mathrm{H}), 5.31(\mathrm{dt}, 1 \mathrm{H}, J=1.2 \mathrm{~Hz}, J=17.2$ $\mathrm{Hz}$ ), $5.26(\mathrm{dq}, 1 \mathrm{H}, J=0.8 \mathrm{~Hz}, J=10.4 \mathrm{~Hz}), 4.69(\mathrm{dd}, 1 \mathrm{H}, J=2.0 \mathrm{~Hz}, J=6.0 \mathrm{~Hz}), 4.40(\mathrm{dd}, 1 \mathrm{H}$, $J=4.0 \mathrm{~Hz}, J=10.0 \mathrm{~Hz}), 4.22(\mathrm{t}, 1 \mathrm{H}, J=6.8 \mathrm{~Hz}), 3.85-3.87(\mathrm{~m}, 2 \mathrm{H}), 3.82(\mathrm{~s}, 3 \mathrm{H}), 3.80-3.81$ $(\mathrm{m}, 1 \mathrm{H}), 2.67-2.72(\mathrm{~m}, 1 \mathrm{H}), 1.94$ (bs, $1 \mathrm{H}) .{ }^{13} \mathbf{C}$ NMR (100 MHz, $\left.\mathbf{C D C l}_{3}\right): \delta 160.14,143.96$, 137.62, 129.46, 127.32, 117.62, 113.65, 101.39, 99.27, 77.98, 75.60, 68.70, 55.26, 42.57. 
18b: ${ }^{1} \mathrm{H}$ NMR (400 MHz, $\left.\mathbf{C D C l}_{3}\right): \delta 7.43(\mathrm{~d}, 2 \mathrm{H}, J=9.6 \mathrm{~Hz}), 6.93(\mathrm{~d}, 2 \mathrm{H}, J=11.7 \mathrm{~Hz}), 6.46$ (dd, $1 \mathrm{H}, J=2.4 \mathrm{~Hz}, J=6.0 \mathrm{~Hz}$ ), 5.93 (ddd, $1 \mathrm{H}, J=4.0 \mathrm{~Hz}, J=10.8 \mathrm{~Hz}, J=17.2 \mathrm{~Hz}$ ), 5.60 (s, $1 \mathrm{H}), 5.38(\mathrm{dt}, 1 \mathrm{H}, J=1.6 \mathrm{~Hz}, J=17.2 \mathrm{~Hz}), 5.26(\mathrm{t}, 1 \mathrm{H}, J=1.6 \mathrm{~Hz}), 4.62(\mathrm{dd}, 1 \mathrm{H}, J=2.0 \mathrm{~Hz}, J$ $=6.0 \mathrm{~Hz}), 4.40(\mathrm{dd}, 1 \mathrm{H}, J=4.0 \mathrm{~Hz}, J=10.0 \mathrm{~Hz}), 4.01(\mathrm{t}, 1 \mathrm{H}, J=9.2 \mathrm{~Hz}), 3.86-3.92(\mathrm{~m}, 2 \mathrm{H})$, $3.82(\mathrm{~s}, 3 \mathrm{H}), 3.77-3.78(\mathrm{~m}, 1 \mathrm{H}), 2.67-2.72(\mathrm{~m}, 1 \mathrm{H}), 1.75(\mathrm{bs}, 1 \mathrm{H}) .{ }^{13} \mathbf{C}$ NMR (100 MHz, $\left.\mathbf{C D C l}_{3}\right)$ : $\delta 160.1,145.5,138.6,129.7,127.4,115.2,113.6,101.4,97.6,75.2,70.1,69.1,55.3,42.4$. 18a and 18b HRMS (ESI-TOF) $\mathrm{m} / \mathrm{z}$ : [M+Na] ${ }^{+}$calcd for $\mathrm{C}_{17} \mathrm{H}_{20} \mathrm{O}_{5} \mathrm{Na} 327.1209$, found 327.1212.

(((2R,3S,4R)-4-(allyloxy)-2-(((tetr-butyldimethylsilyl)oxy)methyl)-3,4-dihydro-2H-pyran3-yl)oxy)(tetr-butyl)dimethylsilane (19)

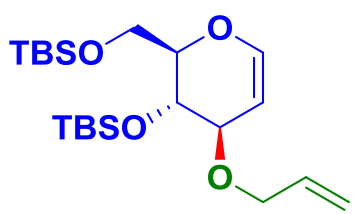

19

Yield: $167 \mathrm{mg}$, 95\%. Rf: 0.7 (5\% EtOAc/hexane). 19: IR (neat): 2923, 2853, 2157, 2008, 1988, 1462, $1253,1101,836,778 \mathrm{~cm}^{-1} .{ }^{1} \mathrm{H}$ NMR (400 MHz, $\left.\mathbf{C D C l}_{3}\right): \delta 6.38(\mathrm{~d}, 1 \mathrm{H}, J=6.0 \mathrm{~Hz})$, 5.90-6.00 (m, $1 \mathrm{H}), 5.30(\mathrm{dq}, 1 \mathrm{H}, J=1.6 \mathrm{~Hz}, J=17.2 \mathrm{~Hz}), 5.18(\mathrm{dq}, 1 \mathrm{H}, J=1.6 \mathrm{~Hz}, J=10.4$ $\mathrm{Hz}$ ), 4.81 (dd, $1 \mathrm{H}, J=2.0 \mathrm{~Hz}, J=6.0 \mathrm{~Hz}$ ), 4.10 (ddt, $1 \mathrm{H}, J=1.2 \mathrm{~Hz}, J=5.6 \mathrm{~Hz}, J=12.0 \mathrm{~Hz}$ ), 3.96 (ddt, $1 \mathrm{H}, J=1.2 \mathrm{~Hz}, J=5.6 \mathrm{~Hz}, J=12.4 \mathrm{~Hz}$ ), 3.86-3.92 (m, 4H), 3.73-3.77 (m, 1H), 0.91 (s, 18H), 0.13 (s, 6H), 0.09 (s, 6H). ${ }^{13} \mathbf{C}$ NMR (100 MHz, $\left.\mathbf{C D C l}_{3}\right): \delta$ 144.6, 134.8, 116.8, 98.9, 79.5, 77.2, 69.2, 67.9, 61.9, 25.9, 25.9, 18.4, 18.2, -4.2, -5.1, -5.1, -5.3. HRMS (ESI-TOF) $\mathrm{m} / \mathrm{z}$ : $[\mathrm{M}+\mathrm{H}]^{+}$calcd for $\mathrm{C}_{21} \mathrm{H}_{43} \mathrm{O}_{4} \mathrm{Si}_{2} 415.2700$, found 415.2639 .

tetr-butyl(((2R,3S,4R)-3-((tetr-butyldimethylsilyl)oxy)-4-(prop-2-yn-1-yloxy)-3,4-dihydro2H-pyran-2-yl)methoxy)dimethylsilane (S20)

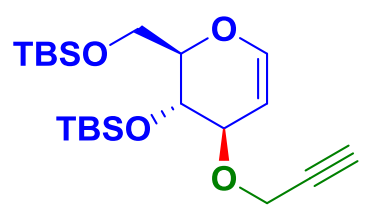

$\mathbf{S 2 0}$

Yield: $160 \mathrm{mg}, 95 \%$. Rf: 0.7 (5\% EtOAc/hexane). S20: IR (neat): 3309, 2953, 2927, 2855, 2077, 2039, 1739, 1648, 1462, 1361, 1252, 1155, 1122, 1099, 1067, 1019, 968, 937, 873, 836, 810, 778, 750, $672 \mathrm{~cm}^{-1}$. ${ }^{1} \mathrm{H}$ NMR (400 MHz, $\left.\mathbf{C D C l}_{3}\right): \delta 6.39$ (dd, $1 \mathrm{H}, J=1.2 \mathrm{~Hz}, J=6.0$ $\mathrm{Hz}$ ), 4.87 (dd, $1 \mathrm{H}, J=2.4 \mathrm{~Hz}, J=6.4 \mathrm{~Hz}$ ), 4.20 (qd, $2 \mathrm{H}, J=2.4 \mathrm{~Hz}, J=15.6 \mathrm{~Hz}$ ), 4.05 (ddd, $1 \mathrm{H}, J=1.6 \mathrm{~Hz}, J=2.0 \mathrm{~Hz}, J=6.4 \mathrm{~Hz}), 3.92(\mathrm{dd}, 1 \mathrm{H}, J=6.0 \mathrm{~Hz}, J=8.4 \mathrm{~Hz}), 3.88-3.89(\mathrm{~m}$, 2H), 3.76-3.80 (m, 1H), $2.43(\mathrm{t}, 1 \mathrm{H}, J=2.4 \mathrm{~Hz}), 0.92(\mathrm{~s}, 9 \mathrm{H}), 0.91(\mathrm{~s}, 9 \mathrm{H}), 0.16(\mathrm{~s}, 3 \mathrm{H}), 0.14$ (s, 3H), 0.08 (s, 3H), 0.07 (s, 3H). $\left.{ }^{13} \mathrm{C} \mathrm{NMR} \mathrm{(100} \mathrm{MHz,} \mathbf{C D C l}_{3}\right): \delta$ 144.9, 98.3, 79.9, 79.5, 76.9, 74.3, 67.7, 61.8, 55.5, 25.9, 18.4, 18.2, -4.1, -5.1, -5.1, -5.3. HRMS (ESI-TOF) $\mathrm{m} / \mathrm{z}:[\mathrm{M}+\mathrm{Na}]^{+}$ calcd for $\mathrm{C}_{21} \mathrm{H}_{40} \mathrm{O}_{4} \mathrm{Si}_{2} \mathrm{Na}$ 435.2363, found 435.2355.

(S)-1-((2R,3S,4S)-3-((tetr-butyldimethylsilyl)oxy)-2-(((tetr-butyldimethylsilyl)oxy)methyl )-3,4-dihydro-2H-pyran-4-yl)prop-2-yn-1-ol (20) 


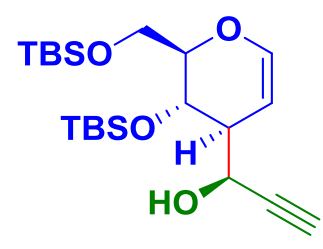

20

Yield: $61 \mathrm{mg}, 55 \%$. Rf: 0.4 (5\% EtOAc/hexane). 20: IR (neat): 2921, 2851, 1738, 1643, 1614, 1518, 1462, 1366, 1231, 1095, 1024, 831, $750 \mathrm{~cm}^{-1}$. ${ }^{1} \mathbf{H}$ NMR (500 MHz, $\left.\mathbf{C D C l}_{3}\right): \delta 6.59$ (dd, $1 \mathrm{H}, J=2.0 \mathrm{~Hz}, J=6.0 \mathrm{~Hz}), 4.83(\mathrm{dd}, 1 \mathrm{H}, J=2.0 \mathrm{~Hz}, J=6.0 \mathrm{~Hz}), 4.60-4.62(\mathrm{~m}, 1 \mathrm{H}), 4.00(\mathrm{dd}$, $1 \mathrm{H}, J=8.5 \mathrm{~Hz}, J=9.5 \mathrm{~Hz}), 3.92(\mathrm{dd}, 1 \mathrm{H}, J=2.0 \mathrm{~Hz}, J=11.5 \mathrm{~Hz}), 3.86(\mathrm{dd}, 1 \mathrm{H}, J=4.0 \mathrm{~Hz}, J$ $=11.5 \mathrm{~Hz}), 3.61(\mathrm{dq}, 1 \mathrm{H}, J=2.0 \mathrm{~Hz}, J=9.5 \mathrm{~Hz}), 2.48-2.51(\mathrm{~m}, 2 \mathrm{H}), 1.75-1.77(\mathrm{~m}, 1 \mathrm{H}), 0.92$ (s, 9H), $0.91(\mathrm{~s}, 9 \mathrm{H}), 0.17(\mathrm{~s}, 3 \mathrm{H}), 0.16(\mathrm{~s}, 3 \mathrm{H}), 0.09(\mathrm{~s}, 3 \mathrm{H}), 0.08(\mathrm{~s}, 3 \mathrm{H}) .{ }^{13} \mathrm{C} \mathrm{NMR}(125 \mathrm{MHz}$, $\left.\mathrm{CDCl}_{3}\right): \delta 147.4,95.9,83.8,79.6,73.3,64.2,62.1,59.8,48.4,25.9,25.9,18.5,18.2,-4.2$, 4.9, -4.9, -5.3. HRMS (ESI-TOF) $\mathrm{m} / \mathrm{z}$ : $[\mathrm{M}+\mathrm{Na}]^{+}$calcd for $\mathrm{C}_{21} \mathrm{H}_{40} \mathrm{O}_{4} \mathrm{Si}_{2} \mathrm{Na} 435.2363$, found 435.2325 .

tert-butyl(((2R,3S,4R)-3-((tert-butyldimethylsilyl)oxy)-4-((4-methylbenzyl)oxy)-3,4-di hydro-2H-pyran-2-yl)methoxy)dimethylsilane (S21)

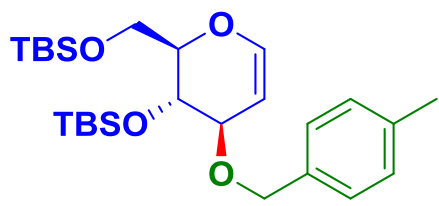

S21

Yield: 170 mg, 94\%, Rf: 0.7 (5\% EtOAc/hexane). S21: IR (neat): 2952, 2927, 2883, 2855, 1714, 1648, 1517, 1462, 1388, 1249, 1151, 1120, 1065, 1007, 969, 938, 874, 835, 808, 778, 738, 704, $673 \mathrm{~cm}^{-1} .{ }^{1} \mathrm{H}$ NMR (500 MHz, $\left.\mathbf{C D C l}_{3}\right): \delta 7.26(\mathrm{~d}, 2 \mathrm{H}, J=8.0 \mathrm{~Hz}), 7.16(\mathrm{~d}, 2 \mathrm{H}, J=$ $8.0 \mathrm{~Hz}), 7.38(\mathrm{~d}, 1 \mathrm{H}, J=6.0 \mathrm{~Hz}), 4.80-4.82(\mathrm{~m}, 1 \mathrm{H}), 4.58(\mathrm{~d}, 1 \mathrm{H}, J=11.0 \mathrm{~Hz}), 4.49(\mathrm{~d}, 1 \mathrm{H}, J$ $=11.5 \mathrm{~Hz}), 3.96-3.99(\mathrm{~m}, 2 \mathrm{H}), 3.91-3.92(\mathrm{~m}, 2 \mathrm{H}), 3.79-3.82(\mathrm{~m}, 1 \mathrm{H}), 2.36(\mathrm{~s}, 3 \mathrm{H}), 0.92(\mathrm{~s}, 9 \mathrm{H})$, 0.90 (s, 9H), 0.12 (s, 3H), 0.11 (s, 3H), 0.09 (s, 3H), 0.08 (s, 3H). $\left.{ }^{13} \mathbf{C ~ N M R ~ ( 1 2 5 ~ M H z , ~} \mathbf{C D C l}_{3}\right)$ : $\delta$ 144.6, 137.1, 135.4, 128.9, 127.8, 99.1, 79.6, 76.8, 70.0, 67.9, 61.9, 25.9, 25.9, 21.1, 18.4, 18.2, -4.2, -5.0, -5.1, -5.3. HRMS (ESI-TOF) $\mathrm{m} / \mathrm{z}$ : $[\mathrm{M}+\mathrm{Na}]^{+}$calcd for $\mathrm{C}_{26} \mathrm{H}_{46} \mathrm{O}_{4} \mathrm{Si}_{2} \mathrm{Na} 501.2832$, found 501.2826 .

(S)-((2R,3S,4S)-3-((tert-butyldimethylsilyl)oxy)-2-(((tert-butyldimethylsilyl)oxy)methyl)3,4-dihydro-2H-pyran-4-yl)(p-tolyl)methanol (21a); (R)-((2R,3S,4S)-3-((tertbutyldimethylsilyl)oxy)-2-(((tert-butyldimethylsilyl)oxy)methyl)-3,4-dihydro-2H-pyran-4yl)(p-tolyl)methanol (21b).
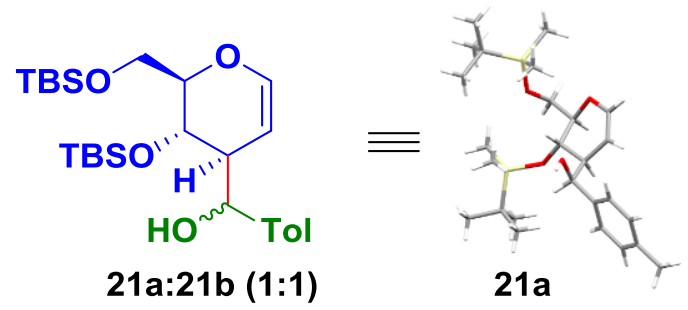
Yield: $42 \mathrm{mg}$, 42\%. Rf: 0.4 (5\% EtOAc/hexane). 21a and 21b: IR (neat): 3497, 2953, 2926, 2855, 1727, 1654, 1513, 1462, 1386, 1360, 1252, 1154, 1114, 1051, 1006, 956, 870, 835, 776, $674 \mathrm{~cm}^{-1} .{ }^{1} \mathbf{H}$ NMR $\left(500 \mathrm{MHz}, \mathbf{C D C l}_{3}\right): \delta 7.25(\mathrm{~d}, 2 \mathrm{H}, J=2.0 \mathrm{~Hz}), 7.24(\mathrm{~d}, 2 \mathrm{H}, J=2.5$ $\mathrm{Hz}), 7.19(\mathrm{~d}, 2 \mathrm{H}, J=7.5 \mathrm{~Hz}), 7.16(\mathrm{~d}, 2 \mathrm{H}, J=8.0 \mathrm{~Hz}), 6.49(\mathrm{dd}, 1 \mathrm{H}, J=2.0 \mathrm{~Hz}, J=6.0 \mathrm{~Hz})$, $6.28(\mathrm{dd}, 1 \mathrm{H}, J=2.0 \mathrm{~Hz}, J=6.5 \mathrm{~Hz}), 5.10(\mathrm{~d}, 1 \mathrm{H}, J=7.5 \mathrm{~Hz}), 4.66(\mathrm{dd}, 1 \mathrm{H}, J=2.0 \mathrm{~Hz}, J=$ $8.0 \mathrm{~Hz}$ ), 4.37 (dd, $1 \mathrm{H}, J=3.5 \mathrm{~Hz}, J=6.0 \mathrm{~Hz}$ ), 4.27 (dd, $1 \mathrm{H}, J=2.0 \mathrm{~Hz}, J=6.0 \mathrm{~Hz}), 4.24$ (t, $1 \mathrm{H}, J=5.5 \mathrm{~Hz}), 4.18(\mathrm{t}, 1 \mathrm{H}, J=9.0 \mathrm{~Hz}), 3.90-3.97(\mathrm{~m}, 2 \mathrm{H}), 3.82-3.88(\mathrm{~m}, 3 \mathrm{H}), 3.60-3.63(\mathrm{~m}$, $1 \mathrm{H}), 2.61(\mathrm{dq}, 1 \mathrm{H}, J=2.5 \mathrm{~Hz}, J=8.5 \mathrm{~Hz}), 2.55-2.58(\mathrm{~m}, 1 \mathrm{H}), 2.37(\mathrm{~s}, 3 \mathrm{H}), 2.36(\mathrm{~s}, 3 \mathrm{H}), 2.31$ (d, $1 \mathrm{H}, J=2.5 \mathrm{~Hz}), 1.78(\mathrm{~d}, 1 \mathrm{H}, J=8.5 \mathrm{~Hz}), 0.98(\mathrm{~s}, 9 \mathrm{H}), 0.95(\mathrm{~s}, 9 \mathrm{H}), 0.94(\mathrm{~s}, 9 \mathrm{H}), 0.90$ (s, $9 \mathrm{H}), 0.24(\mathrm{~s}, 3 \mathrm{H}), 0.22(\mathrm{~s}, 3 \mathrm{H}), 0.21(\mathrm{~s}, 3 \mathrm{H}), 0.20(\mathrm{~s}, 3 \mathrm{H}), 0.11(\mathrm{~s}, 6 \mathrm{H}), 0.09(\mathrm{~s}, 3 \mathrm{H}), 0.05(\mathrm{~s}$, $3 \mathrm{H}) .{ }^{13} \mathrm{C}$ NMR (125 MHz, $\left.\mathrm{CDCl}_{3}\right): \delta$ 146.9, 142.9, 140.2, 139.1, 137.4, 136.5, 128.9, 128.9, 126.9, 125.1, 98.9, 95.2, 79.8, 79.1, 75.6, 69.5, 66.8, 65.2, 62.3, 61.7, 49.4, 47.1, 26.1, 26.0, $25.9,25.8,21.1,21.0,18.5,18.3,18.3,18.3,-3.7,-3.9,-4.1,-4.8,-4.9,-5.3,-5.3,-5.3$. HRMS (ESI-TOF) $\mathrm{m} / \mathrm{z}$ : $[\mathrm{M}+\mathrm{Na}]^{+}$calcd for $\mathrm{C}_{26} \mathrm{H}_{46} \mathrm{O}_{4} \mathrm{Si}_{2} \mathrm{Na} 501.2832$, found 501.2822.

\section{(((2R,3S,4R)-4-(benzyloxy)-2-(((tert-butyldimethylsilyl)oxy)methyl)-3,4-dihydro-2H- pyran-3-yl)oxy)(tert-butyl)dimethylsilane ( $\left.{ }^{1} 15\right)$}

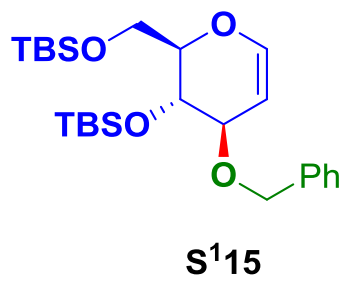

Yield: 186 mg, 93\%, $R_{f}$ : 0.65 (5\% EtOAc/hexane). S'15: IR (neat): 2960, 2952, 2927, 2883, 2855, 1648, 1461, 1388, 1361, 1248, 1098, 1064, 1005, 937, 873, 832, 776, 733, 696, 673 $\mathrm{cm}^{-1} .{ }^{1}{ }^{H}$ NMR (500 MHz, $\left.\mathbf{C D C l}_{3}\right): \delta 7.33-7.38(\mathrm{~m}, 4 \mathrm{H}), 7.28-7.31(\mathrm{~m}, 1 \mathrm{H}), 6.39(\mathrm{~d}, 1 \mathrm{H}, J=6.0$ $\mathrm{Hz}), 4.82(\mathrm{dd}, 1 \mathrm{H}, J=2.5 \mathrm{~Hz}, J=6.0 \mathrm{~Hz}), 4.62(\mathrm{~d}, 1 \mathrm{H}, J=11.5 \mathrm{~Hz}), 4.53(\mathrm{~d}, 1 \mathrm{H}, J=11.5 \mathrm{~Hz})$, 3.97-4.01 (m, 2H), 3.90-3.94 (m, 2H), 3.79-3.83 (m, 1H), $0.92(\mathrm{~s}, 9 \mathrm{H}), 0.90(\mathrm{~s}, 9 \mathrm{H}), 0.12(\mathrm{~s}$, $3 \mathrm{H}), 0.10(\mathrm{~s}, 3 \mathrm{H}), 0.09$ (s, 3H), $\left.0.08(\mathrm{~s}, 3 \mathrm{H}) .{ }^{13} \mathbf{C ~ N M R ~ ( 1 2 5 ~ M H z}, \mathbf{C D C l}_{3}\right): \delta$ 144.7, 138.5, 128.2, 127.7, 127.4, 99.0, 79.6, 76.9, 70.1, 67.9, 61.9, 25.9, 25.9, 18.4, 18.2, -4.2, -5.0, -5.1, -5.3. HRMS (ESI-TOF) $\mathrm{m} / \mathrm{z}$ : [M+Na] ${ }^{+}$calcd for $\mathrm{C}_{25} \mathrm{H}_{44} \mathrm{O}_{4} \mathrm{Si}_{2} \mathrm{Na} 487.2676$, found 487.2672.

tert-butyl(((2R,3S,4R)-3-((tert-butyldimethylsilyl)oxy)-4-(cinnamyloxy)-3,4-dihydro-2Hpyran-2-yl)methoxy)dimethylsilane (S22)

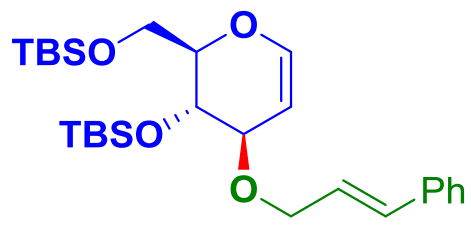

S22

Yield: $184 \mathrm{mg}, 94 \%, \mathrm{R}_{\mathrm{f}}$ : 0.65 (5\% EtOAc/hexane). S22: IR (neat): 2952, 2925, 2853, 1648, 1462, 1388, 1361, 1250, 1120, 1007, 964, 874, 836, 778, 746, $691 \mathrm{~cm}^{-1} .{ }^{1} \mathrm{H}$ NMR (500 MHz, $\left.\mathrm{CDCl}_{3}\right): \delta$ 7.39-7.40 (m, 2H), 7.32-7.35 (m, 2H), 7.24-7.27 (m, $\left.1 \mathrm{H}\right), 6.63(\mathrm{~d}, 1 \mathrm{H}, J=16.0 \mathrm{~Hz})$, $6.40(\mathrm{~d}, 1 \mathrm{H}, J=6.0 \mathrm{~Hz}), 6.32(\mathrm{dt}, 1 \mathrm{H}, J=6.0 \mathrm{~Hz}, J=16.0 \mathrm{~Hz}), 4.85(\mathrm{dd}, 1 \mathrm{H}, J=2.0 \mathrm{~Hz}, J=6.5$ $\mathrm{Hz}$ ), 4.27 (ddd, $1 \mathrm{H}, J=1.5 \mathrm{~Hz}, J=6.0 \mathrm{~Hz}, J=12.0 \mathrm{~Hz}$ ), 4.14 (ddd, $1 \mathrm{H}, J=1.5 \mathrm{~Hz}, J=6.0 \mathrm{~Hz}$, $J=12.5 \mathrm{~Hz}), 3.93-3.96(\mathrm{~m}, 2 \mathrm{H}), 3.91-3.92(\mathrm{~m}, 2 \mathrm{H}), 3.75-3.78(\mathrm{~m}, 1 \mathrm{H}), 0.92(\mathrm{~s}, 9 \mathrm{H}), 0.91(\mathrm{~s}$, 9H), $0.17(\mathrm{~s}, 3 \mathrm{H}), 0.15(\mathrm{~s}, 3 \mathrm{H}), 0.08(\mathrm{~s}, 6 \mathrm{H}) .{ }^{13} \mathbf{C}$ NMR (125 MHz, $\left.\mathbf{C D C l}_{3}\right): \delta$ 144.7, 136.8, $132.1,128.5,127.6,126.4,126.2,99.1,79.6,77.2,68.8,67.9,61.9,25.9,18.5,18.2$, -4.1, - 
5.0, -5.1,-5.3. HRMS (ESI-TOF) $\mathrm{m} / \mathrm{z}$ : $[\mathrm{M}+\mathrm{Na}]^{+}$calcd for $\mathrm{C}_{27} \mathrm{H}_{46} \mathrm{O}_{4} \mathrm{Si}_{2} \mathrm{Na}$ 513.2832, found 513.2828.

(R,E)-1-((2R,3S,4S)-3-((tert-butyldimethylsilyl)oxy)-2-(((tert-butyldimethylsilyl)oxy) methyl)-3,4-dihydro-2H-pyran-4-yl)-3-phenylprop-2-en-1-ol (22)

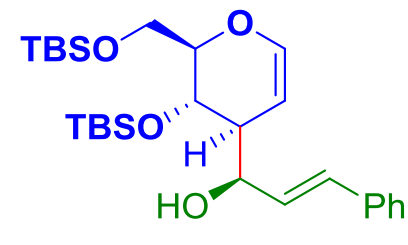

22

Yield: $31 \mathrm{mg}, 62 \%, \mathrm{R}_{\mathrm{f}}$ : 0.35 (5\% EtOAc/hexane). 22: IR (neat): 3478, 3062, 3030, 2952, 2927, 2855, 1730, 1651, 1494, 1462, 1387, 1360, 1252, 1188, 1099, 1055, 1005, 960, 873, 835, 777, 695, $672 \mathrm{~cm}^{-1} .{ }^{1} \mathrm{H}$ NMR (500 MHz, $\left.\mathbf{C D C l}_{3}\right): \delta 7.41$ (d, 2H, $\left.J=7.5 \mathrm{~Hz}\right), 7.34(\mathrm{t}, 2 \mathrm{H}, J=7.5$ $\mathrm{Hz}), 7.24-7.27(\mathrm{~m}, 1 \mathrm{H}), 6.68(\mathrm{dd}, 1 \mathrm{H}, J=1.5 \mathrm{~Hz} J=16.0 \mathrm{~Hz}), 6.55(\mathrm{dd}, 1 \mathrm{H}, J=2.0 \mathrm{~Hz}, J=6.0$ $\mathrm{Hz}), 6.25$ (dd, $1 \mathrm{H}, J=4.0 \mathrm{~Hz}, J=16.0 \mathrm{~Hz}), 4.65-4.66(\mathrm{~m}, 1 \mathrm{H}), 4.53(\mathrm{dd}, 1 \mathrm{H}, J=2.0 \mathrm{~Hz}, J=$ 6.0 Hz), 4.11 (t, $1 \mathrm{H}, J=9.0 \mathrm{~Hz}$ ), 3.88-3.96 (m, 2H), 3.64 (dq, $1 \mathrm{H}, J=2.0 \mathrm{~Hz}, J=9.5 \mathrm{~Hz}), 2.46$ (dq, $1 \mathrm{H}, J=2.0 \mathrm{~Hz}, J=8.0 \mathrm{~Hz}), 1.54(\mathrm{~d}, 1 \mathrm{H}, J=8.5 \mathrm{~Hz}), 0.95(\mathrm{~s}, 9 \mathrm{H}), 0.93(\mathrm{~s}, 9 \mathrm{H}), 0.22(\mathrm{~s}$, $3 \mathrm{H}), 0.20(\mathrm{~s}, 3 \mathrm{H}), 0.11(\mathrm{~s}, 3 \mathrm{H}), 0.10$ (s, 3H). ${ }^{13} \mathbf{C}$ NMR (125 MHz, $\left.\mathbf{C D C l}_{3}\right): \delta$ 147.2, 136.7, 131.5, 129.9, 128.6, 127.5, 126.4, 95.5, 79.9, 68.7, 65.0, 62.3, 47.6, 26.1, 25.9, 18.5, 18.3, 4.1, -4.7, -4.9, -5.3. HRMS (ESI-TOF) $\mathrm{m} / \mathrm{z}$ : $[\mathrm{M}+\mathrm{Na}]^{+}$calcd for $\mathrm{C}_{27} \mathrm{H}_{46} \mathrm{O}_{4} \mathrm{Si}_{2} \mathrm{Na} 513.2832$, found 513.2896 .

(2R,4a $R, 8 R, 8 \mathrm{a} S)-8-($ benzyloxy)-2-(4-methoxyphenyl)-4,4a,8,8a-tetrahydropyrano [3,2-d] $[1,3]$ dioxine (S23)

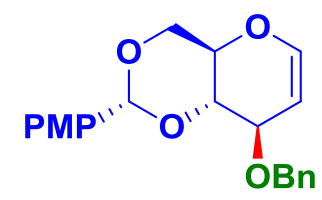

S23

Yield: $128 \mathrm{mg}$, 92\%. Rf: 0.6 (20\% EtOAc/hexane). S23: IR (neat): 3459, 3014, 2968, 2944, 1738, 1437, 1368, 1215, $1095 \mathrm{~cm}^{-1}$. ${ }^{1} \mathrm{H}$ NMR (500 MHz, CDCl $): \delta 7.45$ (d, 2H, J = $\left.8.5 \mathrm{~Hz}\right)$ 7.29-7.39 (m, 5H), $6.93(\mathrm{~d}, 2 \mathrm{H}, J=9.0 \mathrm{~Hz}), 6.37(\mathrm{dd}, 1 \mathrm{H}, J=1.5 \mathrm{~Hz}, J=6.0 \mathrm{~Hz}), 5.62(\mathrm{~s}, 1 \mathrm{H})$, $4.84(\mathrm{dd}, 1 \mathrm{H}, J=2.0 \mathrm{~Hz}, J=6.5 \mathrm{~Hz}), 4.82(\mathrm{~d}, 1 \mathrm{H}, J=12.0 \mathrm{~Hz}), 4.73(\mathrm{~d}, 1 \mathrm{H}, J=12.0 \mathrm{~Hz}), 4.35-$ $4.38(\mathrm{~m}, 2 \mathrm{H}), 4.03(\mathrm{dd}, 1 \mathrm{H}, J=7.5 \mathrm{~Hz}, J=10.0 \mathrm{~Hz}), 3.89-3.94(\mathrm{~m}, 1 \mathrm{H}), 3.85(\mathrm{t}, 1 \mathrm{H}, J=10.0$ $\mathrm{Hz}), 3.84$ (s, 3H). ${ }^{13} \mathbf{C}$ NMR (125 MHz, $\left.\mathbf{C D C l}_{3}\right): \delta 160.1,144.4,138.5,129.9,128.3,127.7$, 127.6, 127.4, 113.6, 102.3, 101.2, 79.9, 73.2, 71.9, 68.7, 68.4, 55.3. HRMS (ESI-TOF) $\mathrm{m} / \mathrm{z}$ : $[\mathrm{M}+\mathrm{Na}]^{+}$calcd for $\mathrm{C}_{21} \mathrm{H}_{22} \mathrm{O}_{5} \mathrm{Na} 377.1365$, found 377.1410 .

(S)-((2R,4a $R, 8 S, 8 \mathrm{a} S)-2-(4-$ methoxyphenyl)-4,4a,8,8a-tetrahydropyrano[3,2-d][1,3]dioxin $-8-y l)($ phenyl)methanol (23)

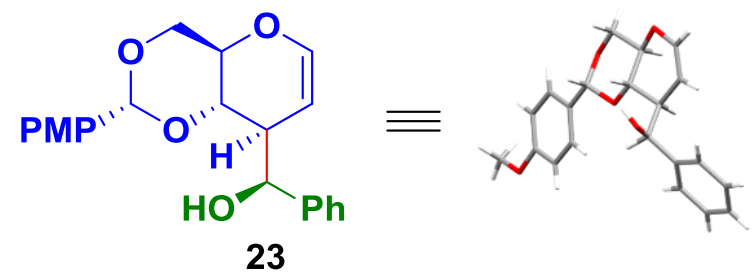


Yield: 45 mg, 50\%, Rf: 0.5 (20\% EtOAc/hexane). 23: IR (neat): 3480, 3065, 2921, 2851, 2213, 2165, 2034, 1974, 1960, 1713, 1641, 1614, 1517, 1493, 1462, 1378, 1303, 1246, 1173, 1129 , 1095, 1027, 990, 938, 827, 746, $706 \mathrm{~cm}^{-1} .{ }^{1} \mathrm{H}$ NMR (500 MHz, $\left.\mathbf{C D C l}_{3}\right): \delta 7.41(\mathrm{~d}, 2 \mathrm{H}, J=8.5$ $\mathrm{Hz}$ ), 7.28-7.40 (m, 5H), $6.92(\mathrm{~d}, 2 \mathrm{H}, J=8.5 \mathrm{~Hz}), 6.43(\mathrm{dd}, 1 \mathrm{H}, J=2.5 \mathrm{~Hz}, J=6.5 \mathrm{~Hz}), 5.60$ (s, $1 \mathrm{H}), 5.07-5.08(\mathrm{~m}, 1 \mathrm{H}), 4.57(\mathrm{dd}, 1 \mathrm{H}, J=2.0 \mathrm{~Hz}, J=6.0 \mathrm{~Hz}), 4.40(\mathrm{dd}, 1 \mathrm{H}, J=5.0 \mathrm{~Hz}, J=$ $10.0 \mathrm{~Hz}$ ), $4.04(\mathrm{t}, 1 \mathrm{H}, J=9.0 \mathrm{~Hz}), 3.88(\mathrm{td}, 1 \mathrm{H}, J=5.0 \mathrm{~Hz}, J=10.0 \mathrm{~Hz}), 3.83(\mathrm{~s}, 3 \mathrm{H}), 3.80(\mathrm{t}$, $1 \mathrm{H}, J=10.0 \mathrm{~Hz}), 2.90(\mathrm{dq}, 1 \mathrm{H}, J=2.5 \mathrm{~Hz}, J=9.5 \mathrm{~Hz}), 2.31$ (bs, $1 \mathrm{H}) .{ }^{13} \mathrm{C}$ NMR (125 MHz, $\left.\mathrm{CDCl}_{3}\right): \delta 160.1,145.4,142.3,129.8,128.3,127.4,127.3,125.7,113.6,101.4,97.7,75.4$, 71.2, 69.3, 68.7, 55.3, 44.6. HRMS (ESI-TOF) $\mathrm{m} / \mathrm{z}$ : $[\mathrm{M}+\mathrm{H}]^{+}$calcd for $\mathrm{C}_{21} \mathrm{H}_{23} \mathrm{O}_{5}$ 355.1546, found 355.1543 .

(2R,4a $R, 8 R, 8 \mathrm{a} S)-2-(4-m e t h o x y p h e n y l)-8-((4-m e t h y l b e n z y l) 0 x y)-4,4 a, 8,8 \mathrm{a}-$ tetrahydro pyrano[3,2-d][1,3]dioxine (S24)

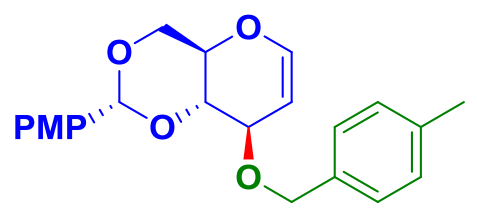

S24

Yield: $168 \mathrm{mg}, 95 \%$. Rf: 0.65 (20\% EtOAc/hexane). S24: IR (neat): 3531, 2921, 2020, 1709, 1642, 1615, $1515 \mathrm{~cm}^{-1} .{ }^{1} \mathrm{H}$ NMR (500 MHz, $\left.\mathbf{C D C l}_{3}\right): \delta 7.42$ (d, 2H, J = 9.0 Hz), 7.24 (d, 2H, J $=8.0 \mathrm{~Hz}), 7.13(\mathrm{~d}, 2 \mathrm{H}, J=8.0 \mathrm{~Hz}), 6.91(\mathrm{~d}, 2 \mathrm{H}, J=9.0 \mathrm{~Hz}), 6.33(\mathrm{dd}, 1 \mathrm{H}, J=1.5 \mathrm{~Hz}, J=6.0$ $\mathrm{Hz}), 5.59(\mathrm{~s}, 1 \mathrm{H}), 4.79(\mathrm{dd}, 1 \mathrm{H}, J=2.0 \mathrm{~Hz}, J=6.0 \mathrm{~Hz}), 4.75(\mathrm{~d}, 1 \mathrm{H}, J=11.5 \mathrm{~Hz}), 4.66(\mathrm{~d}, 1 \mathrm{H}$, $J=11.5 \mathrm{~Hz}$ ), 4.31-4.35 (m, 2H), $4.00(\mathrm{dd}, 1 \mathrm{H}, J=7.5 \mathrm{~Hz}, J=10.0 \mathrm{~Hz}), 3.88(\mathrm{td}, 1 \mathrm{H}, J=5.0$ $\mathrm{Hz}, J=10.0 \mathrm{~Hz}), 3.84(\mathrm{~s}, 1 \mathrm{H}), 3.82(\mathrm{~s}, 3 \mathrm{H}), 2.33(\mathrm{~s}, 3 \mathrm{H}) .{ }^{13} \mathrm{C} \mathrm{NMR}\left(125 \mathrm{MHz}, \mathrm{CDCl}_{3}\right): \delta$ 160.0, 144.3, 137.3, 135.4, 129.8, 129.0, 127.9, 127.4, 113.6, 102.4, 101.2, 79.9, 72.9, 71.9, 68.6, 68.3, 55.3, 21.2. HRMS (ESI-TOF) $\mathrm{m} / \mathrm{z}$ : $[\mathrm{M}+\mathrm{Na}]^{+}$calcd for $\mathrm{C}_{22} \mathrm{H}_{24} \mathrm{O}_{5} \mathrm{Na} 391.1521$, found 391.1516

(S)-((2R,4aR,8S,8aS)-2-(4-methoxyphenyl)-4,4a,8,8a-tetrahydropyrano[3,2-d][1,3]dioxin -8 -yl $)(p$-tolyl)methanol (24)

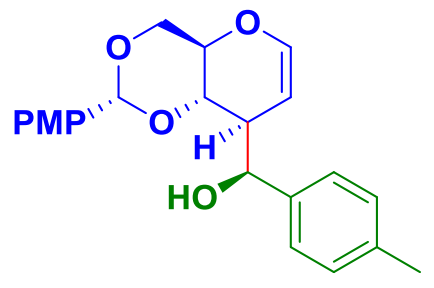

24

Yield: 44 mg, 40\%. Rf: 0.4 (20\% EtOAc/hexane). 24: IR (neat): 3459, 3014, 2969, 2945, 2134, $1738 \mathrm{~cm}$. ${ }^{1} \mathrm{H}$ NMR (400 MHz, $\left.\mathrm{CDCl}_{3}\right): \delta 7.40(\mathrm{~d}, 2 \mathrm{H}, J=8.4 \mathrm{~Hz}), 7.26(\mathrm{~d}, 2 \mathrm{H}, J=8.0 \mathrm{~Hz}), 7.18$ $(\mathrm{d}, 2 \mathrm{H}, J=8.0 \mathrm{~Hz}), 6.92(\mathrm{~d}, 2 \mathrm{H}, J=8.8 \mathrm{~Hz}), 6.28(\mathrm{dd}, 1 \mathrm{H}, J=2.4 \mathrm{~Hz}, J=6.4 \mathrm{~Hz}), 5.63(\mathrm{~s}, 1 \mathrm{H})$, $4.70(\mathrm{~d}, 1 \mathrm{H}, J=8.4 \mathrm{~Hz}), 4.38-4.42(\mathrm{~m}, 1 \mathrm{H}), 4.37(\mathrm{dd}, 1 \mathrm{H}, J=2.0 \mathrm{~Hz}, J=6.4 \mathrm{~Hz}), 3.89-3.91$ $(\mathrm{m}, 2 \mathrm{H}), 3.84(\mathrm{~s}, 3 \mathrm{H}), 3.77-3.83(\mathrm{~m}, 1 \mathrm{H}), 3.37$ (bs, $1 \mathrm{H}), 2.87-2.93(\mathrm{~m}, 1 \mathrm{H}), 2.37(\mathrm{~s}, 3 \mathrm{H}) .{ }^{13} \mathrm{C}$ NMR (100 MHz, $\left.\mathrm{CDCl}_{3}\right)$ : $\delta$ 160.3, 143.8, 138.2, 137.6, 129.3, 128.9, 127.4, 127.1, 113.7, 101.5, 99.3, 79.6, 77.8, 68.8, 68.6, 55.3, 44.2, 21.1. HRMS (ESI-TOF) $\mathrm{m} / \mathrm{z}$ : $[\mathrm{M}+\mathrm{Na}]^{+}$calcd for $\mathrm{C}_{22} \mathrm{H}_{24} \mathrm{O}_{5} \mathrm{Na}$ 391.1522, found 391.1515. 


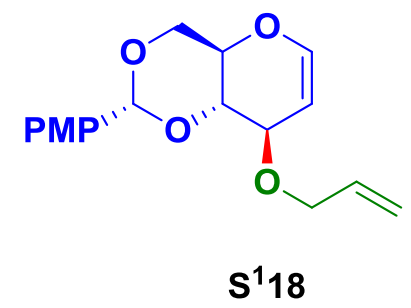

Yield: 164 mg, 80\%, Rf: 0.7 (20\% EtOAc/hexane). S'18: IR (neat): 3073, 2922, 2853, 1713, $1641,1615,1587,1517,1463,1423,1372,1303,1283,1250,1234,1171,1124,1098,1070$, 1035, 1003, 926, 828, 782, $736 \mathrm{~cm}^{-1}$. ${ }^{1} \mathrm{H}$ NMR (500 MHz, $\left.\mathbf{C D C l}_{3}\right): \delta 7.34$ (dd, $2 \mathrm{H}, J=2.0 \mathrm{~Hz}$, $J=8.5 \mathrm{~Hz}$ ), 6.82 (dd, $2 \mathrm{H}, J=2.0 \mathrm{~Hz}, J=9.0 \mathrm{~Hz}$ ), 6.26 (dd, $1 \mathrm{H}, J=1.5 \mathrm{~Hz}, J=6.0 \mathrm{~Hz}$ ), $5.80-$ $5.88(\mathrm{~m}, 1 \mathrm{H}), 5.50(\mathrm{~s}, 1 \mathrm{H}), 5.23(\mathrm{dd}, 1 \mathrm{H}, J=2.0 \mathrm{~Hz}, J=12.5 \mathrm{~Hz}), 5.09(\mathrm{dd}, 1 \mathrm{H}, J=2.0 \mathrm{~Hz}, J$ $=10.5 \mathrm{~Hz}$ ), $4.72(\mathrm{dd}, 1 \mathrm{H}, J=2.0 \mathrm{~Hz}, J=6.5 \mathrm{~Hz}), 4.26(\mathrm{dd}, 1 \mathrm{H}, J=5.0 \mathrm{~Hz}, J=10.5 \mathrm{~Hz}), 4.16-$ $4.21(\mathrm{~m}, 2 \mathrm{H}), 4.06-4.10(\mathrm{~m}, 1 \mathrm{H}), 3.86(\mathrm{dd}, 1 \mathrm{H}, J=7.5 \mathrm{~Hz}, J=10.0 \mathrm{~Hz}), 3.81(\mathrm{td}, 1 \mathrm{H}, J=4.5$ $\mathrm{Hz}, J=10.0 \mathrm{~Hz}), 3.73(\mathrm{t}, 1 \mathrm{H}, J=10.0 \mathrm{~Hz}), 3.72(\mathrm{~s}, 3 \mathrm{H}) .{ }^{13} \mathbf{C}$ NMR (125 MHz, $\left.\mathbf{C D C l}_{3}\right): \delta$ 160.0, 144.3, 134.9, 129.7, 127.3, 116.9, 113.5, 102.2, 101.1, 79.8, 73.0, 70.9, 68.6, 68.3, 55.2. HRMS (ESI-TOF) $\mathrm{m} / \mathrm{z}$ : [M+Na] ${ }^{+}$calcd for $\mathrm{C}_{17} \mathrm{H}_{20} \mathrm{O}_{5} \mathrm{Na} 327.1208$, found 327.1248.

(2R,4a $R, 8 R, 8 \mathrm{a} S)-8$-(cinnamyloxy)-2-(4-methoxyphenyl)-4,4a,8,8a-tetrahydro pyrano [3,2-d][1,3]dioxine (S25)<smiles>CC(C)[Pb]C1OC[C@H]2OC=CC(OC/C=C\c3ccccc3)[C@H]2O1</smiles>

S25

Yield: $186 \mathrm{mg}, 95 \%$. Rf: 0.5 (20\% EtOAc/hexane). S25: IR (neat): 2921, 2851, 1716, 1654, 1614, 1517, 1452, 1303, 1248, 1171, 1099, 1030, 830, 772, 747, $697 \mathrm{~cm}^{-1} .{ }^{1} \mathbf{H}$ NMR (500 MHz, $\left.\mathrm{CDCl}_{3}\right): \delta 7.45-7.48(\mathrm{~m}, 2 \mathrm{H}), 7.23-7.36(\mathrm{~m}, 5 \mathrm{H}), 6.91-6.94(\mathrm{~m}, 2 \mathrm{H}), 6.64(\mathrm{~d}, 1 \mathrm{H}, J=16.0 \mathrm{~Hz})$, $6.39(\mathrm{dd}, 1 \mathrm{H}, J=1.5 \mathrm{~Hz}, J=6.0 \mathrm{~Hz}), 6.31(\mathrm{dt}, 1 \mathrm{H}, J=6.0 \mathrm{~Hz}, J=16.0 \mathrm{~Hz}), 5.63(\mathrm{~s}, 1 \mathrm{H}), 4.86$ (dd, $1 \mathrm{H}, J=2.0 \mathrm{~Hz}, J=6.5 \mathrm{~Hz}$ ), 4.44 (ddd, $1 \mathrm{H}, J=1.5 \mathrm{~Hz}, J=6.0 \mathrm{~Hz}, J=13.0 \mathrm{~Hz}$ ), 4.37-4.39 $(\mathrm{m}, 2 \mathrm{H}), 4.35-4.36(\mathrm{~m}, 1 \mathrm{H}), 4.00(\mathrm{dd}, 1 \mathrm{H}, J=7.5 \mathrm{~Hz}, J=10.0 \mathrm{~Hz}), 3.93(\mathrm{td}, 1 \mathrm{H}, J=5.0 \mathrm{~Hz}, J$ $=10.0 \mathrm{~Hz}), 3.85(\mathrm{t}, 1 \mathrm{H}, J=10.0 \mathrm{~Hz}), 3.84(\mathrm{~s}, 3 \mathrm{H}) \cdot{ }^{13} \mathbf{C}$ NMR $\left(125 \mathbf{~ M H z}, \mathbf{C D C l}_{3}\right): \delta 160.1,144.4$, 136.7, 132.3, 129.7, 128.5, 127.6, 127.4, 126.5, 126.1, 113.6, 102.2, 101.3, 79.8, 73.0, 70.5, 68.6, 68.3, 55.3. HRMS (ESI-TOF) $\mathrm{m} / \mathrm{z}$ : $[\mathrm{M}+\mathrm{H}]^{+}$calcd for $\mathrm{C}_{23} \mathrm{H}_{25} \mathrm{O}_{5} 381.1703$, found 381.1705.

$(R, E)-1-((2 R, 4 \mathrm{a} R, 8 S, 8 \mathrm{aS})-2-(4-m e t h o x y p h e n y l)-4,4 \mathrm{a}, 8,8 \mathrm{a}-$ tetrahydropyrano[3,2-d][1,3] dioxin-8-yl)-3-phenylprop-2-en-1-ol (25)

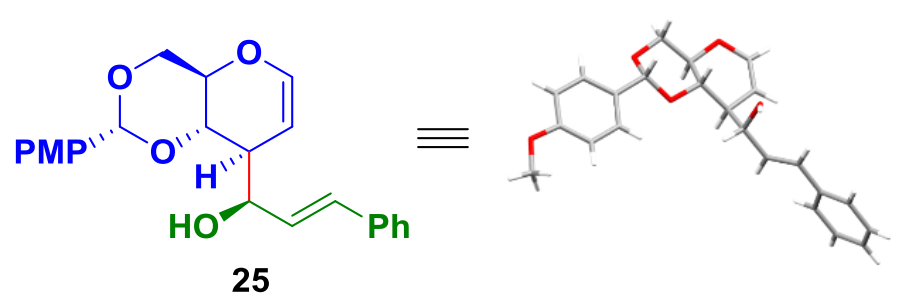


Yield: 66 mg, 60\%. Rf: 0.6 (30\% EtOAc/hexane). 25: IR (neat): 3329, 2878, 1642, 1613, 1518, 1369, 1234, 1087, 1018, 961, 817, 749, 695, $662 \mathrm{~cm}^{-1} .{ }^{1} \mathbf{H}$ NMR (400 MHz, $\left.\mathbf{C D C l}_{3}\right): \delta 7.40-$ $7.43(\mathrm{~m}, 4 \mathrm{H}), 7.26-7.36(\mathrm{~m}, 3 \mathrm{H}), 6.89-6.91(\mathrm{~m}, 2 \mathrm{H}), 6.73(\mathrm{dd}, 1 \mathrm{H}, J=1.6 \mathrm{~Hz}, J=16.0 \mathrm{~Hz})$, $6.48(\mathrm{dd}, 1 \mathrm{H}, J=2.4 \mathrm{~Hz}, J=6.0 \mathrm{~Hz}), 6.31(\mathrm{dd}, 1 \mathrm{H}, J=4.8 \mathrm{~Hz}, J=16.0 \mathrm{~Hz}), 5.60(\mathrm{~s}, 1 \mathrm{H}), 4.73$ (dd, $1 \mathrm{H}, J=2.0 \mathrm{~Hz}, J=6.0 \mathrm{~Hz}$ ), $4.60(\mathrm{bs}, 1 \mathrm{H}), 4.42(\mathrm{dd}, 1 \mathrm{H}, J=4.8 \mathrm{~Hz}, J=10.4 \mathrm{~Hz}$ ), 4.03 (t, $1 \mathrm{H}, J=9.2 \mathrm{~Hz}), 3.88-3.95(\mathrm{~m}, 1 \mathrm{H}), 3.83(\mathrm{~s}, 3 \mathrm{H}), 3.81(\mathrm{t}, 1 \mathrm{H}, J=10.4 \mathrm{~Hz}), 2.78-2.82(\mathrm{~m}, 1 \mathrm{H})$, 2.07 (bs, $1 \mathrm{H}) .{ }^{13} \mathrm{C}$ NMR (100 MHz, $\left.\mathbf{C D C l}_{3}\right): \delta 160.1,145.4,136.6,130.5,130.1,129.7,128.6$, 127.6, 127.4, 126.5, 113.6, 101.5, 98.1, 75.5, 70.8, 69.1, 68.7, 55.3, 43.0. HRMS (ESI-TOF) $\mathrm{m} / \mathrm{z}:[\mathrm{M}+\mathrm{H}]^{+}$calcd for $\mathrm{C}_{23} \mathrm{H}_{25} \mathrm{O}_{5} 381.1703$, found 381.1706 .

(2R,4a $R, 8 R, 8 \mathrm{a} S)-8-($ allyloxy)-2-phenyl-4,4a,8,8a-tetrahydropyrano[3,2-d][1,3] dioxine (S26)

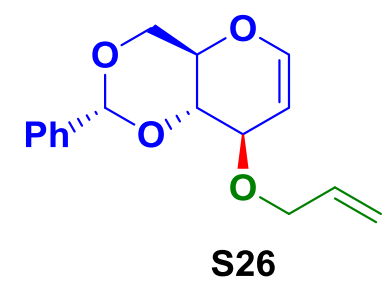

Yield: 148 mg, 98\%. Rf: 0.65 (20\% EtOAc/hexane). S26: IR (neat): 2922, 2868, 1643, 1452, $1371,1283,1263,1233,1162,1125,1100,1070,996,919,874,829,748,696 \mathrm{~cm}^{-1} .{ }^{1} \mathbf{H}$ NMR $\left(500 \mathrm{MHz}, \mathrm{CDCl}_{3}\right): \delta$ 7.51-7.54 (m, 2H), 7.36-7.42 (m, 3H), $6.37(\mathrm{dd}, 1 \mathrm{H}, J=1.4 \mathrm{~Hz}, J=6.1$ $\mathrm{Hz}), 5.92-5.99(\mathrm{~m}, 1 \mathrm{H}), 5.65(\mathrm{~s}, 1 \mathrm{H}), 5.32(\mathrm{dq}, 1 \mathrm{H}, J=1.6 \mathrm{~Hz}, J=17.2 \mathrm{~Hz}), 5.20(\mathrm{dq}, 1 \mathrm{H}, J=$ $1.3 \mathrm{~Hz}, J=10.4 \mathrm{~Hz}), 4.83(\mathrm{dd}, 1 \mathrm{H}, J=2.0 \mathrm{~Hz}, J=6.2 \mathrm{~Hz}), 4.39(\mathrm{dd}, 1 \mathrm{H}, J=4.7 \mathrm{~Hz} J=10.2$ $\mathrm{Hz}), 4.28-4.33(\mathrm{~m}, 2 \mathrm{H}), 4.18-4.22(\mathrm{~m}, 1 \mathrm{H}), 3.99(\mathrm{dd}, 1 \mathrm{H}, J=7.2 \mathrm{~Hz}, J=10.0 \mathrm{~Hz}), 3.90-3.95$ $(\mathrm{m}, 1 \mathrm{H}), 3.86(\mathrm{t}, 1 \mathrm{H}, J=10.0 \mathrm{~Hz}) .{ }^{13} \mathrm{C}$ NMR $\left(125 \mathrm{MHz}, \mathbf{C D C l}_{3}\right): \delta 144.3,137.3,134.9,129.0$,

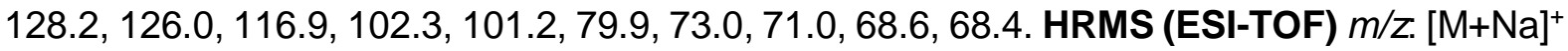
calcd for $\mathrm{C}_{16} \mathrm{H}_{18} \mathrm{O}_{4} \mathrm{Na} 297.1103$, found 297.1102.

(R/S)-1-((2R,4aR,8S,8aS)-2-phenyl-4,4a,8,8a-tetrahydropyrano[3,2-d][1,3]dioxin-8yl)prop-2-en-1-ol (26a \&26b)<smiles>C=C[C@H](O)[C@H]1C=CO[C@@H]2CO[C@H](c3ccccc3)O[C@H]21</smiles>

26a:26b (4:1)

Yield: $54 \mathrm{mg}, 60 \%$, $\mathrm{R}_{\mathrm{f}}$ : 0.4 (20\% EtOAc/hexane). IR (neat): 3447, 2863, 1640, 1454, 1379, 1291, 1238, 1163, 1091, 1007, 922, 863, 752, $697 \mathrm{~cm}^{-1}$. 26a: ${ }^{1} \mathbf{H}$ NMR (500 MHz, $\left.\mathbf{C D C l}_{3}\right): \delta$ 7.49-7.53 (m, 1H), 7.38-7.43 (m, 4H), $6.47(\mathrm{dd}, 1 \mathrm{H}, J=2.5 \mathrm{~Hz}, J=6.5 \mathrm{~Hz}), 5.92-5.98(\mathrm{~m}, 1 \mathrm{H})$, $5.66(\mathrm{~s}, 1 \mathrm{H}), 5.40(\mathrm{dt}, 1 \mathrm{H}, J=1.5 \mathrm{~Hz}, J=17.0 \mathrm{~Hz}), 5.27-5.30(\mathrm{~m}, 1 \mathrm{H}), 4.64(\mathrm{dd}, 1 \mathrm{H}, J=2.0$ $\mathrm{Hz}, J=6.5 \mathrm{~Hz}$ ), $4.48(\mathrm{bs}, 1 \mathrm{H}), 4.43(\mathrm{dd}, 1 \mathrm{H}, J=5.0 \mathrm{~Hz}, J=10.0 \mathrm{~Hz}), 4.04(\mathrm{t}, 1 \mathrm{H}, J=9.5 \mathrm{~Hz})$, 3.88-3.93 (m, $1 \mathrm{H}), 3.83(\mathrm{t}, 1 \mathrm{H}, J=10.5 \mathrm{~Hz}), 2.72(\mathrm{dq}, 1 \mathrm{H}, J=2.5 \mathrm{~Hz}, J=9.5 \mathrm{~Hz}), 1.85(\mathrm{bs}$, 1H). ${ }^{13} \mathrm{C}$ NMR (125 MHz, $\left.\mathbf{C D C l}_{3}\right): \delta 145.5,138.6,137.3,129.1,128.3,126.1,115.3,101.5$, 97.7, 75.4, 70.2, 69.2, 68.8, 42.5. 26b: ${ }^{1} \mathrm{H}$ NMR (500 MHz, $\left.\mathbf{C D C l}_{3}\right)$ : $\delta 7.49-7.53(\mathrm{~m}, 1 \mathrm{H}), 7.38-$ $7.43(\mathrm{~m}, 4 \mathrm{H}), 6.40(\mathrm{dd}, 1 \mathrm{H}, J=2.5 \mathrm{~Hz}, J=6.5 \mathrm{~Hz}), 5.86-5.93(\mathrm{~m}, 1 \mathrm{H}), 5.65(\mathrm{~s}, 1 \mathrm{H}), 5.32$ (dt, $1 \mathrm{H}, J=1.5 \mathrm{~Hz}, J=17.0 \mathrm{~Hz}), 5.26-5.28(\mathrm{~m}, 1 \mathrm{H}), 4.71(\mathrm{dd}, 1 \mathrm{H}, J=2.0 \mathrm{~Hz}, J=6.5 \mathrm{~Hz}), 4.43$ (dd, $1 \mathrm{H}, J=5.0 \mathrm{~Hz}, J=10.0 \mathrm{~Hz}$ ), $4.24(\mathrm{t}, 1 \mathrm{H}, J=7.0 \mathrm{~Hz}), 4.14(\mathrm{q}, 1 \mathrm{H}, J=7.0 \mathrm{~Hz}), 3.88-3.93$ (m, $1 \mathrm{H}), 3.82(\mathrm{t}, 1 \mathrm{H}, J=10.5 \mathrm{~Hz}), 2.72(\mathrm{dq}, 1 \mathrm{H}, J=2.5 \mathrm{~Hz}, J=9.5 \mathrm{~Hz}), 2.61(\mathrm{bs}, 1 \mathrm{H}) .{ }^{13} \mathrm{C} \mathrm{NMR}$ (125 MHz, $\left.\mathrm{CDCl}_{3}\right)$ : $\delta 144.1,137.7,137.0,129.2,128.3,126.0,117.6,101.5,99.3,78.0,75.6$, 
68.8, 68.8, 42.7. HRMS (ESI-TOF) $\mathrm{m} / \mathrm{z}$ : $[\mathrm{M}+\mathrm{Na}]^{+}$calcd for $\mathrm{C}_{16} \mathrm{H}_{18} \mathrm{O}_{4} \mathrm{Na}$ 297.1103, found 297.1106.

(2R,4a $R, 8 R, 8 \mathrm{a} S)-8-($ cinnamyloxy)-2-phenyl-4,4a,8,8a-tetrahydropyrano[3,2-d][1,3] dioxine (S27)<smiles>C1=CC(OC/C=C/c2ccccc2)[C@H]2O[C@H](c3ccccc3)OC[C@H]2O1</smiles>

S27

Yield: 168 mg, 98\%, Rf: 0.65 (20\% EtOAc/hexane). S27: IR (neat): 3029, 2855, 1639, 1494, 1451, 1378, 1279, 1231, 1165, 1096, 1070, 966, 914, 874, 823, 747, $695 \mathrm{~cm}^{-1}$. ${ }^{1} \mathrm{H}$ NMR (500 $\left.\mathbf{M H z}, \mathrm{CDCl}_{3}\right): \delta 7.26-7.57(\mathrm{~m}, 10 \mathrm{H}), 6.65(\mathrm{~d}, 1 \mathrm{H}, J=16.0 \mathrm{~Hz}), 6.40(\mathrm{dd}, 1 \mathrm{H}, J=1.6 \mathrm{~Hz}, J=6.1$ $\mathrm{Hz}), 6.30-6.35(\mathrm{~m}, 1 \mathrm{H}), 5.68(\mathrm{~s}, 1 \mathrm{H}) 4.87(\mathrm{dd}, 1 \mathrm{H}, J=2.0 \mathrm{~Hz}, J=6.1 \mathrm{~Hz}), 4.46$ (ddd, $1 \mathrm{H}, J=$ $1.5 \mathrm{~Hz}, J=5.8 \mathrm{~Hz}, J=12.9 \mathrm{~Hz}), 4.36-4.42(\mathrm{~m}, 3 \mathrm{H}), 4.03(\mathrm{dd}, 1 \mathrm{H}, J=7.3 \mathrm{~Hz}, J=10.0 \mathrm{~Hz})$, 3.93-3.98 (m, 1H), $3.88(\mathrm{t}, 1 \mathrm{H}, J=10.2 \mathrm{~Hz}) .{ }^{13} \mathbf{C}$ NMR (125 MHz, CDCl $): \delta$ 144.4, 137.3, 136.7, 132.3, 129.0, 128.5, 128.2, 127.6, 126.5, 126.1, 102.3, 101.3, 79.9, 73.0, 70.6, 68.6, 68.4. HRMS (ESI-TOF) $\mathrm{m} / \mathrm{z}$ : [M+Na] ${ }^{+}$calcd for $\mathrm{C}_{22} \mathrm{H}_{22} \mathrm{O}_{4} \mathrm{Na} 373.1416$, found 373.1412.

$(R, E)-3-p h e n y l-1-((2 R, 4 a R, 8 S, 8 a S)-2-p h e n y l-4,4 a, 8,8 a-t e t r a h y d r o p y r a n o[3,2-d][1,3]$ dioxin-8-yl)prop-2-en-1-ol (27)

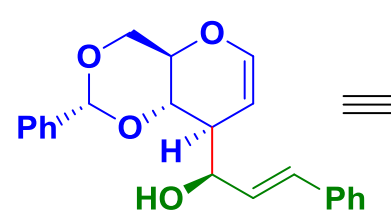

27

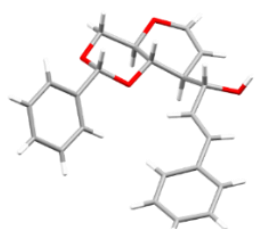

Yield: $68 \mathrm{mg}, 60 \%$. Rf: 0.5 (20\% Acetone/hexane). 27: IR (neat): 3291, 3062, 2868, 1640, 1492, 1449, 1366, 1343, 1313, 1236, 1158, 1127, 1085, 1019, 962, 927, 743, $694 \mathrm{~cm}^{-1} .{ }^{1} \mathbf{H}$ NMR (500 MHz, $\left.\mathbf{C D C l}_{3}\right): \delta 7.26-7.51(\mathrm{~m}, 10 \mathrm{H}), 6.73(\mathrm{dd}, 1 \mathrm{H}, J=1.5 \mathrm{~Hz}, J=16.0 \mathrm{~Hz}), 6.49$ (dd, $1 \mathrm{H}, J=2.4 \mathrm{~Hz}, J=6.0 \mathrm{~Hz}$ ), 6.32 (dd, $1 \mathrm{H}, J=5.0 \mathrm{~Hz}, J=16.0 \mathrm{~Hz}$ ), 5.65 (s, 1H), 4.74 (dd, $1 \mathrm{H}, J=2.0 \mathrm{~Hz}, J=6.0 \mathrm{~Hz}), 4.61(\mathrm{bs}, 1 \mathrm{H}), 4.44(\mathrm{dd}, 1 \mathrm{H}, J=5.0 \mathrm{~Hz}, J=10 \mathrm{~Hz}), 4.05(\mathrm{t}, 1 \mathrm{H}, J=$ $9 \mathrm{~Hz}), 3.90-3.95(\mathrm{~m}, 1 \mathrm{H}), 3.84(\mathrm{t}, 1 \mathrm{H}, J=10 \mathrm{~Hz}), 2.79-2.82(\mathrm{~m}, 1 \mathrm{H}), 2.07(\mathrm{~s}, 1 \mathrm{H}) .{ }^{13} \mathrm{C}$ NMR (125 MHz, $\mathrm{CDCl}_{3}$ ): $\delta 145.3,137.2,136.6,130.6,130.2,129.1,128.6,128.3,127.7,126.5$, 126.1, 101.5, 98.2, 75.6, 70.9, 69.2, 68.8, 43.1. HRMS (ESI-TOF) $\mathrm{m} / \mathrm{z}$ : [M+Na] ${ }^{+}$calcd for $\mathrm{C}_{22} \mathrm{H}_{22} \mathrm{O}_{4} \mathrm{Na} 373.1416$, found 373.1414.

(((2R,3S,4S)-4-(allyloxy)-2-(((tetr-butyldimethylsilyl)oxy)methyl)-3,4-dihydro-2H-pyran3-yl)oxy)(tetr-butyl)dimethylsilane (28)

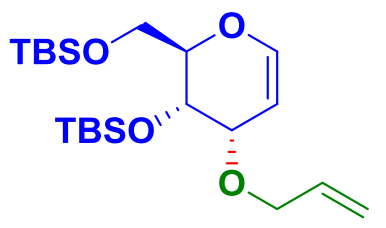

28

Yield: 146 mg, 72\%. Rf: 0.8 (5\% EtOAc/hexane). 28: IR (neat): 2953, 2928, 2892, 2856, 1642 $\mathrm{cm}^{-1} .{ }^{1} \mathrm{H}$ NMR $\left(500 \mathrm{MHz}, \mathrm{CDCl}_{3}\right): \delta 6.35(\mathrm{~d}, 1 \mathrm{H}, J=5.5 \mathrm{~Hz}), 5.90-5.98(\mathrm{~m}, 1 \mathrm{H}), 5.28(\mathrm{dq}, 1 \mathrm{H}$, $J=1.5 \mathrm{~Hz}, J=17.5 \mathrm{~Hz}), 5.16(\mathrm{dq}, 1 \mathrm{H}, J=1.5 \mathrm{~Hz}, J=10.5 \mathrm{~Hz}), 4.77(\mathrm{t}, 1 \mathrm{H}, J=5.5 \mathrm{~Hz}), 4.26$ 
(dd, $1 \mathrm{H}, J=3.5 \mathrm{~Hz}, J=6.0 \mathrm{~Hz}$ ), 4.18 (ddt, $1 \mathrm{H}, J=1.5 \mathrm{~Hz}, J=6.0 \mathrm{~Hz}, J=12.5 \mathrm{~Hz}$ ), 3.99-4.05 (m, 2H), 3.89-3.95 (m, 2H), $3.60(\mathrm{dd}, 1 \mathrm{H}, J=3.5 \mathrm{~Hz}, J=9.5 \mathrm{~Hz}), 0.92(\mathrm{~s}, 9 \mathrm{H}), 0.90(\mathrm{~s}, 9 \mathrm{H})$, $0.10(\mathrm{~s}, 6 \mathrm{H}), 0.09$ (s, 3H), $0.08(\mathrm{~s}, 3 \mathrm{H}) .{ }^{13} \mathbf{C}$ NMR (125 MHz, $\left.\mathbf{C D C l}_{3}\right): \delta$ 145.2, 134.9, 116.7, 101.3, 73.7, 73.6, 70.2, 62.1, 60.9, 25.9, 25.9, 18.4, 18.2, -4.1, -4.5, -5.2, -5.4. HRMS (ESITOF) $\mathrm{m} / \mathrm{z}:[\mathrm{M}+\mathrm{K}]^{+}$calcd for $\mathrm{C}_{21} \mathrm{H}_{42} \mathrm{O}_{4} \mathrm{Si}_{2} \mathrm{~K} 453.2259$, found 453.2267 .

(R)-1-((2R,3S,4R)-3-((tetr-butyldimethylsilyl)oxy)-2-(((tetr-butyldimethylsilyl)oxy) methyl)-3,4-dihydro-2H-pyran-4-yl)prop-2-en-1-ol (29)

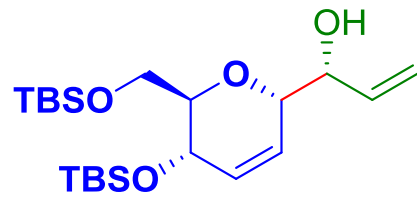

29

Yield: 70 mg, 64\%. Rf: 0.3 (5\% EtOAc/hexane). 29: IR (neat): 3425, 2954, 2929, 2887, 2856, $1718 \mathrm{~cm}^{-1}$. ${ }^{1} \mathrm{H}$ NMR (500 MHz, $\left.\mathbf{C D C l}_{3}\right): \delta 5.99$ (ddd, $1 \mathrm{H}, J=5.0 \mathrm{~Hz}, J=10.5 \mathrm{~Hz}, J=17.0 \mathrm{~Hz}$ ), 5.94 (ddd, $1 \mathrm{H}, J=2.5 \mathrm{~Hz}, J=3.5 \mathrm{~Hz}, J=10.0 \mathrm{~Hz}$ ), 5.83 (ddd, $1 \mathrm{H}, J=1.5 \mathrm{~Hz}, J=2.5 \mathrm{~Hz} J=$ $10.5 \mathrm{~Hz}$ ), 5.39 (dt, $1 \mathrm{H}, J=1.5 \mathrm{~Hz}, J=17.5 \mathrm{~Hz}$ ), $5.23(\mathrm{dt}, 1 \mathrm{H}, J=1.5 \mathrm{~Hz}, J=10.5 \mathrm{~Hz}), 4.32$ $(\mathrm{m}, 1 \mathrm{H}), 4.15-4.17(\mathrm{~m}, 1 \mathrm{H}), 4.08-4.10(\mathrm{~m}, 1 \mathrm{H}), 3.74-3.78(\mathrm{~m}, 1 \mathrm{H}), 3.67-3.72(\mathrm{~m}, 2 \mathrm{H}), 2.48$ (bs, $1 \mathrm{H}), 0.92(\mathrm{~s}, 9 \mathrm{H}), 0.91(\mathrm{~s}, 9 \mathrm{H}), 0.11(\mathrm{~s}, 3 \mathrm{H}), 0.10(\mathrm{~s}, 3 \mathrm{H}), 0.07(\mathrm{~s}, 3 \mathrm{H}), 0.06(\mathrm{~s}, 3 \mathrm{H}) .{ }^{13} \mathrm{C}$ NMR (125 MHz, $\mathbf{C D C l}_{3}$ ): $\delta 137.2,130.4,126.0,115.9,77.1,74.4,74.2,63.3,62.8,25.9,25.8,18.3$, 18.0, -4.3, -4.8, -5.3, -5.4. HRMS (ESI-TOF) $\mathrm{m} / \mathrm{z}$ : $[\mathrm{M}+\mathrm{Na}]^{+}$calcd for $\mathrm{C}_{21} \mathrm{H}_{42} \mathrm{O}_{4} \mathrm{Si}_{2} \mathrm{Na} 437.2519$, found 437.2515 .

tert-butyl(((2R,3S,4S)-3-((tetr-butyldimethylsilyl)oxy)-4-(cinnamyloxy)-3,4-dihydro-2Hpyran-2-yl)methoxy)dimethylsilane (S30)

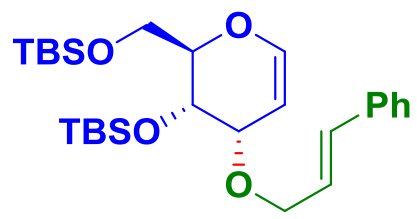

S30

Yield: $184 \mathrm{mg}, 74 \%, \mathrm{R}_{\mathrm{f}}: 0.7$ (5\% EtOAc/hexane). S30: IR (neat): 3577, 3419, 2954, 2928, 2897, 2855, $1642 \mathrm{~cm}^{-1}$. ${ }^{1} \mathrm{H}$ NMR (500 MHz, $\left.\mathbf{C D C l}_{3}\right): \delta$ 7.24-7.41 (m, 5H), $6.62(\mathrm{~d}, 1 \mathrm{H}, J=16.0$ $\mathrm{Hz}), 6.44(\mathrm{~d}, 1 \mathrm{H}, J=6.0 \mathrm{~Hz}), 6.34(\mathrm{dt}, 1 \mathrm{H}, J=6.0 \mathrm{~Hz}, J=16.0 \mathrm{~Hz}), 4.91(\mathrm{t}, 1 \mathrm{H}, J=6.0 \mathrm{~Hz})$, 4.26-435 (m, 2H), 4.06 (dd, $1 \mathrm{H}, J=2.5 \mathrm{~Hz}, J=10.0 \mathrm{~Hz}$ ), 4.01 (dd, $1 \mathrm{H}, J=3.0 \mathrm{~Hz}, J=10.0$ $\mathrm{Hz}), 3.90-3.96(\mathrm{~m}, 2 \mathrm{H}), 3.83(\mathrm{dd}, 1 \mathrm{H}, J=4.0 \mathrm{~Hz}, J=5.5 \mathrm{~Hz}), 0.96(\mathrm{~s}, 9 \mathrm{H}), 0.92(\mathrm{~s}, 9 \mathrm{H}), 0.16$ (s, 3H), $0.14(\mathrm{~s}, 3 \mathrm{H}), 0.10$ (s, 3H), 0.09 (s, 3H). ${ }^{13} \mathbf{C}$ NMR (125 MHz, $\mathbf{C D C l}_{3}$ ): $\delta$ 146.3, 136.9, 131.8, 128.5, 127.5, 127.0, 126.4, 98.3, 75.2, 70.1, 69.6, 68.1, 62.3, 25.9, 25.9, 18.5, 18.2, 4.2, -4.9, -5.0, -5.3. HRMS (ESI-TOF) $\mathrm{m} / \mathrm{z}$ : $[\mathrm{M}+\mathrm{Na}]^{+}$calcd for $\mathrm{C}_{27} \mathrm{H}_{46} \mathrm{O}_{4} \mathrm{Si}_{2} \mathrm{Na}$ 513.2833, found 513.2847.

(S,E)-1-((2S,5S,6R)-5-((tert-butyldimethylsilyl)oxy)-6-(((tert-butyldimethylsilyl)oxy) methyl)-5,6-dihydro-2H-pyran-2-yl)-3-phenylprop-2-en-1-ol (30) 


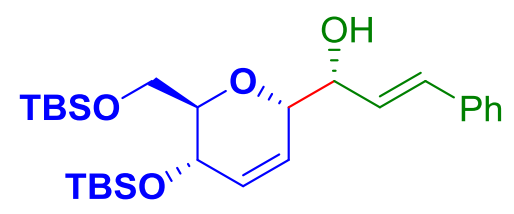

30

Yield: 42 mg, 80\%, $\mathrm{R}_{\mathrm{f}}$ : 0.3 (5\% EtOAc/hexane). 30: IR (neat): 3427, 3027, 2953, 2929, 2885, 2856, $1580 \mathrm{~cm}^{-1}$. ${ }^{1} \mathrm{H}$ NMR (500 MHz, $\left.\mathbf{C D C l}_{3}\right): \delta 7.38-7.41(\mathrm{~m}, 5 \mathrm{H}), 6.72(\mathrm{~d}, 1 \mathrm{H}, J=16.0 \mathrm{~Hz})$, $6.34(\mathrm{dd}, 1 \mathrm{H}, J=5.5 \mathrm{~Hz}, J=16.0 \mathrm{~Hz}$ ), 5.98 (ddd, $1 \mathrm{H}, J=2.5 \mathrm{~Hz}, J=3.5 \mathrm{~Hz}, J=10.5 \mathrm{~Hz}$ ), $5.88(\mathrm{dt}, 1 \mathrm{H}, J=1.5 \mathrm{~Hz}, J=10.5 \mathrm{~Hz}), 4.52(\mathrm{td}, 1 \mathrm{H}, J=1.0 \mathrm{~Hz}, J=5.0 \mathrm{~Hz}), 4.26-4.28(\mathrm{~m}, 1 \mathrm{H})$, $4.13(\mathrm{~d}, 1 \mathrm{H}, J=1.0 \mathrm{~Hz}), 3.70-3.79(\mathrm{~m}, 3 \mathrm{H}), 2.71(\mathrm{bs}, 1 \mathrm{H}), 0.93(\mathrm{~s}, 9 \mathrm{H}), 0.91(\mathrm{~s}, 9 \mathrm{H}), 0.13(\mathrm{~s}$, $3 \mathrm{H}), 0.12(\mathrm{~s}, 3 \mathrm{H}), 0.06$ (s, 6H). ${ }^{13} \mathrm{C}$ NMR (125 MHz, $\left.\mathbf{C D C l}_{3}\right): \delta 136.8,131.0,130.3,128.5$, 128.5, 127.5, 126.5, 126.2, 77.3, 74.6, 73.9, 63.2, 62.7, 25.9, 25.8, 18.3, 18.0, -4.4, -4.8, -5.3, -5.4. HRMS (ESI-TOF) $\mathrm{m} / \mathrm{z}$ : [M+K] ${ }^{+}$calcd for $\mathrm{C}_{27} \mathrm{H}_{46} \mathrm{O}_{4} \mathrm{Si}_{2} \mathrm{~K} 529.2572$, found 529.2570.

(2R,4a $R, 8 S, 8 \mathrm{aS})$-8-(cinnamyloxy)-2-(4-methoxyphenyl)-4,4a,8,8a-tetrahydro pyrano [3,2-d][1,3]dioxine (S31)<smiles>O=[Pb]C1OC[C@H]2OC=C[C@H](OC/C=C\c3ccccc3)[C@@H]2O1</smiles>

S31

Yield: $166 \mathrm{mg}$, 98\%. Rf: 0.6 (20\% EtOAc/hexane). S31: IR (neat): 3435, 3061, 3024, 2964, 2931, 2906, 2859, 2245, 2032, 1636, 1614, 1588, $1517 \mathrm{~cm}^{-1} .{ }^{1} \mathbf{H}$ NMR (500 MHz, $\left.\mathbf{C D C l}_{3}\right): \delta$ $7.49(\mathrm{~d}, 2 \mathrm{H}, J=8.5 \mathrm{~Hz}), 7.24-7.39(\mathrm{~m}, 5 \mathrm{H}), 6.94(\mathrm{~d}, 2 \mathrm{H}, J=9.0 \mathrm{~Hz}), 6.63(\mathrm{~d}, 1 \mathrm{H}, J=16.0 \mathrm{~Hz})$, $6.44(\mathrm{~d}, 1 \mathrm{H}, J=6.0 \mathrm{~Hz}), 6.36(\mathrm{dt}, 1 \mathrm{H}, J=6.0 \mathrm{~Hz}, J=16.0 \mathrm{~Hz}), 5.57(\mathrm{~s}, 1 \mathrm{H}), 5.02(\mathrm{t}, 1 \mathrm{H}, J=6.0$ $\mathrm{Hz}$ ), 4.54 (ddd, $1 \mathrm{H}, J=1.0 \mathrm{~Hz}, J=5.5 \mathrm{~Hz}, J=13.0 \mathrm{~Hz}$ ), 4.47 (dd, $1 \mathrm{H}, J=5.5 \mathrm{~Hz}, J=10.5 \mathrm{~Hz}$ ), 4.30-4.38 (m, 2H), $4.07(\mathrm{dd}, 1 \mathrm{H}, J=3.5 \mathrm{~Hz}, J=6.0 \mathrm{~Hz}), 3.96(\mathrm{dd}, 1 \mathrm{H}, J=3.5 \mathrm{~Hz}, J=10.5$ $\mathrm{Hz})$, 3.82-3.86 (m, 1H), 3.84(s, 3H). $\left.{ }^{13} \mathbf{C ~ N M R ~ ( 1 2 5 ~ M H z , ~} \mathbf{C D C l}_{3}\right): \delta 160.1,145.6,136.8,131.9$, 129.9, 128.4, 127.5, 127.5, 126.8, 126.4, 113.6, 101.9, 100.2, 79.2, 71.7, 68.7, 67.1, 64.4, 55.2. HRMS (ESI-TOF) $\mathrm{m} / \mathrm{z}$ : $[\mathrm{M}+\mathrm{H}]^{+}$calcd for $\mathrm{C}_{23} \mathrm{H}_{25} \mathrm{O}_{5} 381.1703$, found 381.1700 .

$(S, E)-1-((2 R, 4 a R, 6 S, 8 \mathrm{a} S)-2-(4-m e t h o x y p h e n y l)-4,4 a, 6,8 \mathrm{a}-$ tetrahydropyrano[3,2-d][1,3] dioxin-6-yl)-3-phenylprop-2-en-1-ol (31)

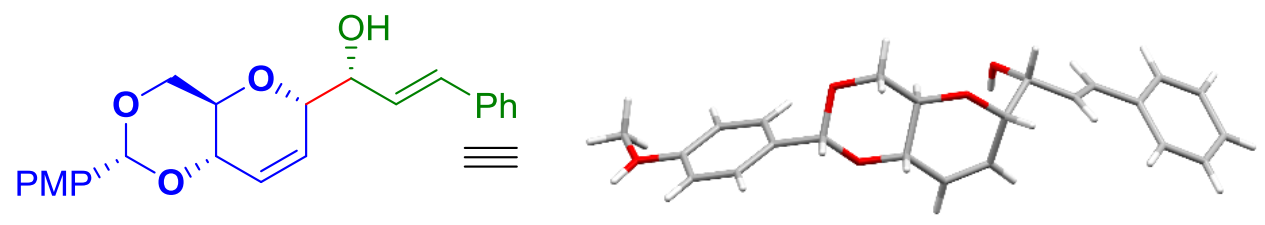

31

Yield: $77 \mathrm{mg}, 70 \%$. $\mathrm{R}_{\mathrm{f}}$ : 0.5 (30\% EtOAc/hexane). 31: IR (neat): 3443, 2960, 2909, 2881, 2840, 2805, 1613, $1517 \mathrm{~cm}^{-1}$. ${ }^{1} \mathrm{H}$ NMR (400 MHz, $\left.\mathbf{C D C l}_{3}\right): \delta$ 7.41-7.46 (m, 4H), 7.34-7.38 (m, 2H), 7.27-7.31 (m, 1H), $6.91(\mathrm{~d}, 2 \mathrm{H}, J=8.8 \mathrm{~Hz}), 6.72(\mathrm{dd}, 1 \mathrm{H}, J=1.2 \mathrm{~Hz}, J=16.0 \mathrm{~Hz}), 6.33$ (dd, $1 \mathrm{H}, J=6.0 \mathrm{~Hz}, J=16.0 \mathrm{~Hz}), 6.20(\mathrm{bd}, 1 \mathrm{H}, J=10.4 \mathrm{~Hz}), 5.95(\mathrm{dt}, 1 \mathrm{H}, J=2.4 \mathrm{~Hz}, J=10.8 \mathrm{~Hz})$, $5.57(\mathrm{~s}, 1 \mathrm{H}), 4.49-4.53(\mathrm{~m}, 1 \mathrm{H}), 4.34-4.38(\mathrm{~m}, 1 \mathrm{H}), 4.31-4.33(\mathrm{~m}, 1 \mathrm{H}), 4,14-4.16(\mathrm{~m}, 1 \mathrm{H}), 3.82$ (s, 3H), 3.70-3.79 (m, 2H), $2.20(\mathrm{~d}, 1 \mathrm{H}, J=5.6 \mathrm{~Hz}) .{ }^{13} \mathrm{C}$ NMR (100 MHz, $\left.\mathbf{C D C l}_{3}\right): \delta$ 160.1, 136.4, 131.6, 129.9, 129.3, 128.6, 128.2, 127.9, 127.5, 126.6, 125.9, 113.7, 101.8, 76.6, 75.1, 
74.7, 69.6, 67.3, 55.3. HRMS (ESI-TOF) $\mathrm{m} / \mathrm{z}$ : $[\mathrm{M}+\mathrm{H}]^{+}$calcd for $\mathrm{C}_{23} \mathrm{H}_{25} \mathrm{O}_{5}$ 381.1703, found 381.1701.

(((2R,3S,4S)-4-(benzyloxy)-2-((tert-butyldimethylsilyl)oxy)methyl)-3,4-dihydro-2Hpyran-3-yl)oxy)(tert-butyl)dimethylsilane ( $\left.{ }^{1} 4\right)$

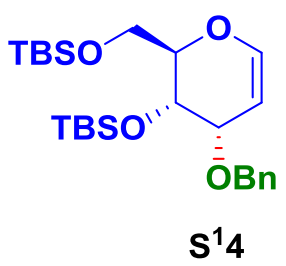

Yield: 148 mg, 76\%. Rf: 0.8 (5\% EtOAc/hexane). S'4: IR (neat): 2960, 2928, 2892, 2855, 1724, $1642 \mathrm{~cm}^{-1}$. ${ }^{1} \mathrm{H}$ NMR (500 MHz, $\left.\mathbf{C D C l}_{3}\right): \delta 7.27-7.39(\mathrm{~m}, 5 \mathrm{H}), 6.36(\mathrm{~d}, 1 \mathrm{H}, J=6.0 \mathrm{~Hz}), 4.78$ (t, $1 \mathrm{H}, J=6.6 \mathrm{~Hz}), 4.74(\mathrm{~d}, 1 \mathrm{H}, J=12.0 \mathrm{~Hz}), 4.55(\mathrm{~d}, 1 \mathrm{H}, J=11.5 \mathrm{~Hz}), 4.31$ (dd, $1 \mathrm{H} . J=3.0 \mathrm{~Hz}$, $J=5.5 \mathrm{~Hz}$ ), 4.09 (dt, $1 \mathrm{H}, J=3.0 \mathrm{~Hz}, J=10.0 \mathrm{~Hz}$ ), 3.95 (d, $2 \mathrm{H}, J=3.5 \mathrm{~Hz}), 3.69(\mathrm{dd}, 1 \mathrm{H}, J=$ $3.5 \mathrm{~Hz}, J=10.0 \mathrm{~Hz}), 0.91(\mathrm{~s}, 9 \mathrm{H}), 0.90(\mathrm{~s}, 9 \mathrm{H}), 0.09(\mathrm{~s}, 6 \mathrm{H}), 0.08(\mathrm{~s}, 3 \mathrm{H}), 0.07(\mathrm{~s}, 3 \mathrm{H}) .{ }^{13} \mathrm{C}$ NMR (100 MHz, $\left.\mathbf{C D C l}_{3}\right): \delta 145.2,138.5,128.2,127.7,127.4,101.3,74.1,73.8,71.2,62.3$, 60.9, 25.9, 25.9, 18.4, 18.2, -4.0, -4.4, -5.2, -5.3. HRMS (ESI-TOF) $\mathrm{m} / \mathrm{z}$ : [M+Na] ${ }^{+}$calcd for $\mathrm{C}_{25} \mathrm{H}_{44} \mathrm{O}_{4} \mathrm{Si}_{2} \mathrm{Na}$ 487.2676, found 487.2673.

tert-butyl(((2R,3S,4S)-3-((tert-butyldimethylsilyl)oxy)-4-((4-methylbenzyl)oxy)-3,4-di hydro-2H-pyran-2-yl)methoxy)dimethylsilane ( $\left.{ }^{1} 6\right)$

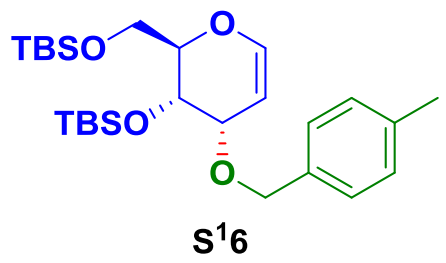

Yield: 158 mg, 75\%. Rf: 0.7 (5\% EtOAc/hexane). S'6: IR (neat): 3582, 2957, 2928, 2894, 2856, $1642 \mathrm{~cm}^{-1}$. ${ }^{1} \mathrm{H}$ NMR (500 MHz, $\left.\mathbf{C D C l}_{3}\right): \delta 7.27(\mathrm{~d}, 2 \mathrm{H}, J=7.0 \mathrm{~Hz}), 7.15(\mathrm{~d}, 2 \mathrm{H}, J=7.5 \mathrm{~Hz})$, $6.42(\mathrm{~d}, 1 \mathrm{H}, J=5.5 \mathrm{~Hz}), 4.87(\mathrm{t}, 1 \mathrm{H}, J=5.5 \mathrm{~Hz}), 4.64(\mathrm{t}, 2 \mathrm{H}, J=12.5 \mathrm{~Hz}), 4.08-4.11(\mathrm{~m}, 1 \mathrm{H})$, $3.98(\mathrm{dd}, 1 \mathrm{H}, J=3.5 \mathrm{~Hz}, J=10.5 \mathrm{~Hz}), 3.94(\mathrm{dd}, 1 \mathrm{H}, J=2.0 \mathrm{~Hz}, J=11.5 \mathrm{~Hz}), 3.89$ (dd, $1 \mathrm{H}, J$ $=4.0 \mathrm{~Hz}, J=11.5 \mathrm{~Hz}$ ), $3.81(\mathrm{dd}, 1 \mathrm{H}, J=4.0 \mathrm{~Hz}, J=6.0 \mathrm{~Hz}), 2.35(\mathrm{~s}, 3 \mathrm{H}), 0.94(\mathrm{~s}, 9 \mathrm{H}), 0.92$ (s, 9H), 0.12 (s, 3H), $0.11(\mathrm{~s}, 3 \mathrm{H}), 0.09$ (s, 3H), 0.08 (s, 3H). $\left.{ }^{13} \mathbf{C ~ N M R ~ ( 1 0 0 ~ M H z , ~} \mathbf{C D C l}_{3}\right): \delta$ 146.2, 136.9, 135.9, 128.9, 127.8, 98.4, 75.2, 70.5, 69.8, 68.2, 62.3, 25.9, 25.9, 21.1, 18.5, 18.1, -4.1, -4.9, -5.0, -5.3. HRMS (ESI-TOF) $\mathrm{m} / \mathrm{z}$ : $[\mathrm{M}+\mathrm{Na}]^{+}$calcd for $\mathrm{C}_{26} \mathrm{H}_{46} \mathrm{O}_{4} \mathrm{Si}_{2} \mathrm{Na} 501.2832$, found 501.2855 .

(2S,4a $R, 8 S, 8 \mathrm{a} R)-8$-(cinnamyloxy)-2-(4-methoxyphenyl)-4,4a,8,8a-tetrahydro pyrano [3,2-d][1,3]dioxine (S32)

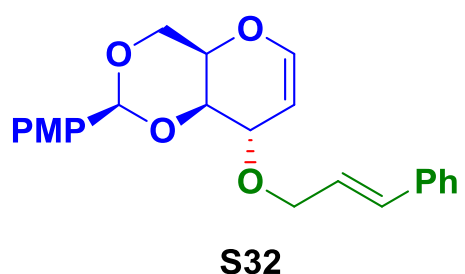

Yield: $188 \mathrm{mg}, 92 \%$. Rf: 0.5 (30\% EtOAc/hexane). S32: IR (neat): 3450, 2920, 2852, 1713, $1655,1614,1516,1450,1393,1365,1302,1246,1170,1097,1070,1029,885,829,773$, 737, $695 \mathrm{~cm}^{-1} .{ }^{1} \mathrm{H}$ NMR (500 MHz, $\left.\mathbf{C D C l}_{3}\right): \delta 7.48(\mathrm{dd}, 2 \mathrm{H}, J=2.0 \mathrm{~Hz}, J=6.5 \mathrm{~Hz}), 7.38-7.39$ 
$(\mathrm{m}, 2 \mathrm{H}), 7.32-7.35(\mathrm{~m}, 2 \mathrm{H}), 7.25-7.27(\mathrm{~m}, 1 \mathrm{H}), 6.88(\mathrm{dd}, 2 \mathrm{H}, J=2.0 \mathrm{~Hz}, J=6.5 \mathrm{~Hz}), 6.65(\mathrm{~d}$, $1 \mathrm{H}, J=16.0 \mathrm{~Hz}$ ), $6.50(\mathrm{dd}, 1 \mathrm{H}, J=2.0 \mathrm{~Hz}, J=6.5 \mathrm{~Hz}), 6.35(\mathrm{dt}, 1 \mathrm{H}, J=6.0 \mathrm{~Hz}, J=16.0 \mathrm{~Hz})$, $5.63(\mathrm{~s}, 1 \mathrm{H}), 4.83(\mathrm{dt}, 1 \mathrm{H}, J=1.5 \mathrm{~Hz}, J=6.5 \mathrm{~Hz}), 4.43-4.44(\mathrm{~m}, 1 \mathrm{H}), 4.34-4.38(\mathrm{~m}, 2 \mathrm{H}), 4.33$ (dd, $2 \mathrm{H}, J=1.5 \mathrm{~Hz}, J=6.0 \mathrm{~Hz}$ ), 4.05 (dd, $1 \mathrm{H}, J=1.0 \mathrm{~Hz}, J=12.5 \mathrm{~Hz}$ ), 3.90 (s, $1 \mathrm{H}$ ), 3.81 (s, 3H). ${ }^{13} \mathrm{C}$ NMR (125 MHz, $\left.\mathbf{C D C l}_{3}\right): \delta$ 160.0, 144.1, 136.5, 132.4, 130.3, 128.5, 127.7, 126.4, 126.2, 113.5, 101.2, 99.2, 70.8, 69.6, 69.5, 68.9, 67.9, 55.2. HRMS (ESI-TOF) $\mathrm{m} / \mathrm{z}:[\mathrm{M}+\mathrm{H}]^{+}$ calcd for $\mathrm{C}_{23} \mathrm{H}_{25} \mathrm{O}_{5} 381.1703$, found 381.1704 .

$(R, E)-1-((2 S, 4 a R, 8 R, 8 \mathrm{a} R)-2-(4-m e t h o x y p h e n y l)-4,4 \mathrm{a}, 8,8 \mathrm{a}-t e t r a h y d r o p y r a n o[3,2-\mathrm{d}][1,3]$ dioxin-8-yl)-3-phenylprop-2-en-1-ol (32)

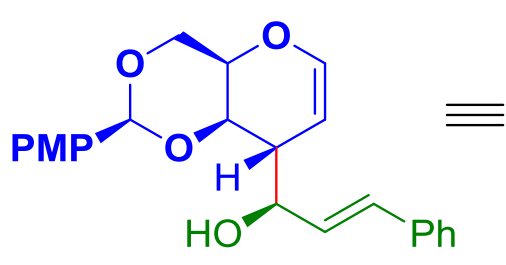

32

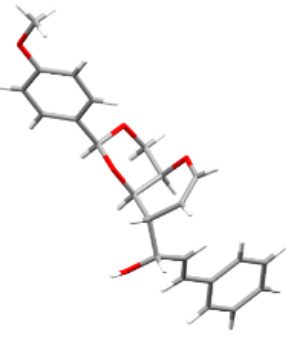

Yield: 52 mg, 58\%, Rf: 0.45 (30\% EtOAc/hexane). 32: IR (neat): 3450, 2921, 2852, 1652, $1614,1517,1494,1448,1394,1334,1304,1247,1172,1092,1075,1029,976,931,879$, 829, 771, 755, $696 \mathrm{~cm}^{-1} .{ }^{1} \mathbf{H}$ NMR (400 MHz, $\left.\mathbf{C D C l}_{3}\right)$ : $\delta 7.31-7.47(\mathrm{~m}, 7 \mathrm{H}), 6.90(\mathrm{dd}, 2 \mathrm{H}, \mathrm{J}=$ $2.0 \mathrm{~Hz}, J=6.8 \mathrm{~Hz}), 6.73(\mathrm{dd}, 1 \mathrm{H}, J=0.8 \mathrm{~Hz}, J=6.0 \mathrm{~Hz}), 6.65(\mathrm{dd}, 1 \mathrm{H}, J=0.8 \mathrm{~Hz}, J=16.0$ $\mathrm{Hz}), 6.30(\mathrm{dd}, 1 \mathrm{H}, J=5.6 \mathrm{~Hz}, J=16.0 \mathrm{~Hz}), 5.62(\mathrm{~s}, 1 \mathrm{H}), 4.68-4.71(\mathrm{~m}, 1 \mathrm{H}), 4.40-4.42(\mathrm{~m}, 1 \mathrm{H})$, $4.36(\mathrm{dd}, 1 \mathrm{H}, J=1.6 \mathrm{~Hz}, J=12.4 \mathrm{~Hz}), 4.25(\mathrm{~s}, 1 \mathrm{H}), 4.09(\mathrm{~d}, 1 \mathrm{H}, J=12.4 \mathrm{~Hz}), 3.91(\mathrm{~s}, 1 \mathrm{H})$, 3.81 (s, 3H), 2.52 (s, 1H), 1.86 (bs, $1 \mathrm{H}) .{ }^{13} \mathbf{C}$ NMR (100 MHz, $\left.\mathbf{C D C l}_{3}\right): \delta$ 160.1, 145.9, 136.2, 130.8, 130.4, 130.1, 128.7, 127.9, 127.5, 126.5, 113.6, 101.4, 94.7, 74.9, 73.9, 69.4, 67.3, 55.3, 42.4. HRMS (ESI-TOF) $\mathrm{m} / \mathrm{z}$ : $[\mathrm{M}+\mathrm{H}]^{+}$calcd for $\mathrm{C}_{23} \mathrm{H}_{25} \mathrm{O}_{5} 381.1703$, found 381.1706.

\section{X-ray Crystal Structure and Details of all the following Compounds}

The absolute configuration of products $3,12 b, 16 a, 21 a, 23,25,27,31$ and 32 were determined by X-ray crystallography analysis. Single crystal of 3, 12b, 16a, 21a, 23, 25, 27, $\mathbf{3 1}$ and $\mathbf{3 2}$ were recrystallized from mixed solvents of Ethyl acetate and Hexane respectively. These data can be obtained free of charge via www.ccdc.cam.ac.uk/data request/cif or by emailing data request@ccdc.cam.ac.uk. X-ray reflections were collected on Bruker D8 QUEST, Rigaku Oxford diffraction XtaLAB Synergy, CCD diffractometer equipped with a graphite monochromator and Mo Ka fine-focus sealed tube $(\lambda=0.71073 \AA)$ and reduction was performed using APEXIII Software. Intensities were corrected for absorption using SADABS, and the structure was solved and refined using SHELX2018. All nonhydrogen atoms were refined anisotropically. Hydrogen atoms on heteroatoms were located from difference electron-density maps and all $\mathrm{C}-\mathrm{H}$ hydrogen atoms were fixed geometrically. Hydrogen-bond geometries were determined in PLATON. 
Crystal data and structure refinement for 3: CCDC: 2068610

Crystal system

Crystal size

Bond precision:

Cell:

Temperature:

Volume

Space group

Hall group

Moiety formula

Sum formula

M. Wt

Dx,g cm-3

Z

$\mathrm{Mu}(\mathrm{mm}-1)$

F000

F000'

$\mathrm{h}, \mathrm{k}, \operatorname{Imax}$

Nref

Data completeness $=1.42 / 0.75$

$\mathrm{R}$ (reflections) $=0.0589(2527)$

$S=1.059$

Compound 3 (CCDC: 2068610); The ellipsoid contour \% probability levels in the caption for the image of 3 was $50 \%$.

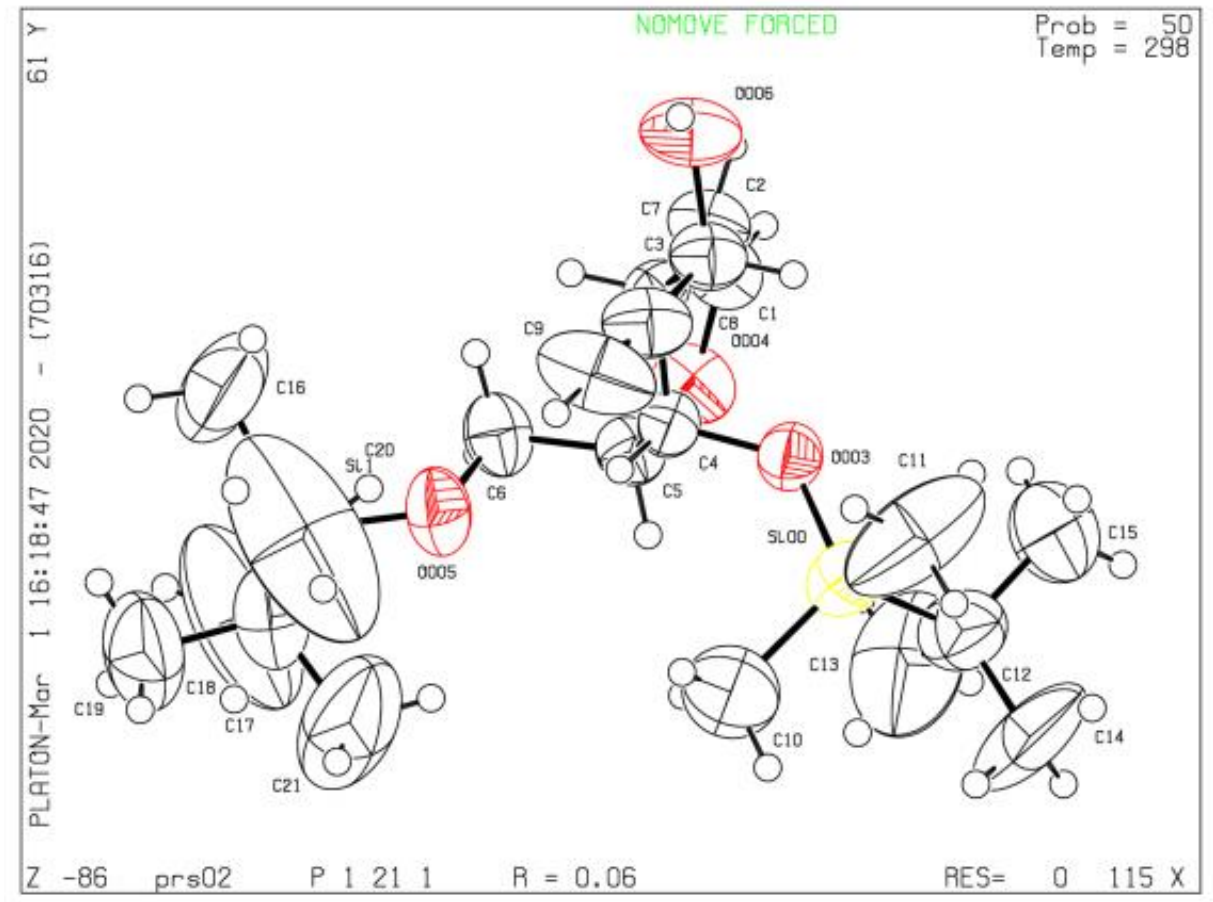

Wavelength $=0.71073 \AA$

$b=12.0033(5) \AA$

$\beta=89.993(4)^{\circ}$

$\mathrm{c}=13.8446(6) \AA$

$\mathrm{Y}=90^{\circ}$

Reported

1316.83(9) $\AA^{3}$

P 1211

$\mathrm{P} 2 \mathrm{yb}$

$\mathrm{C} 21 \mathrm{H} 40 \mathrm{O} 4 \mathrm{Si} 2$

$\mathrm{C} 21 \mathrm{H} 40 \mathrm{O} 4 \mathrm{Si} 2$

412.71

1.041

2

0.154

452.0

$9,14,16$

3470

Theta $(\max )=25.015$

wR2 (reflections) $=0.1796(3470$ )

Npar $=259$
( 3 was $50 \%$
Calculated

0.154

452.50

$9,14,16$

4641 [ 2442] 
Crystal data and structure refinement for 12b: CCDC: 2068619

Crystal system

Crystal size

Bond precision:

Cell:

Temperature:

Volume

Space group

Hall group

Empirical formula

M. Wt

Dx, g cm-3

Z

$\mathrm{Mu}(\mathrm{mm}-1)$

F000

F000'

$\mathrm{h}, \mathrm{k}, \mathrm{Imax}$

Nref

Data completeness $=1.99 / 1.00$

$\mathrm{R}$ (reflections) $=0.0364(2934)$

$\mathrm{S}=1.034$

Compound 12b (CCDC: 2068619); The ellipsoid contour \% probability levels in the caption for the image of $12 b$ was $50 \%$.

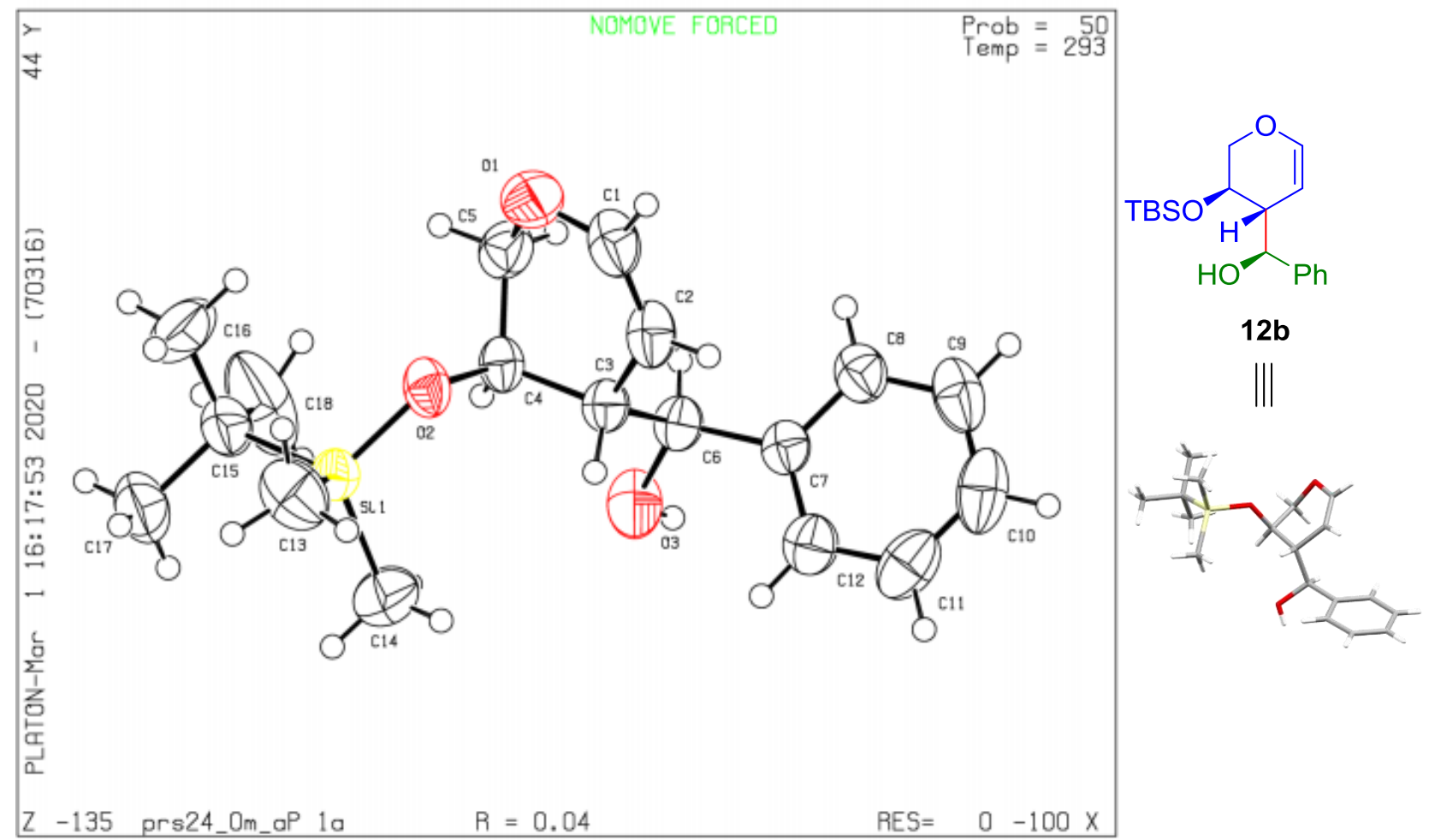

Wavelength $=0.71073 \AA$

$\mathrm{C}=8.9077(5) \AA$

$Y=97.718(3)^{\circ}$

Reported

464.28(5) $\AA^{3}$

P 1

P 1

$\mathrm{C} 18 \mathrm{H} 28 \mathrm{O} 3 \mathrm{Si}$

320.49

1.146

1

0.136

174.0

$8,8,10$

3265

Theta $(\max )=25.023$

wR2 $($ reflections $)=0.0841(3265)$

Npar $=205$
174.15

$8,8,10$ 
Crystal data and structure refinement for 16a: CCDC: 2068621

Crystal system

Crystal size

Bond precision:

Cell:

Temperature:

Volume

Space group

Hall group

Empirical formula

M. Wt

Dx, g cm-3

Z

$\mathrm{Mu}(\mathrm{mm}-1)$

F000

F000'

h,k,Imax

Nref

Data completeness $=1.76 / 0.99$

$\mathrm{R}($ reflections $)=0.0430(6216)$

$S=1.046$

$293 \mathrm{~K}$

1.110

4

0.104
Orthorhombic

$0.20 \times 0.15 \times 0.10$

$\mathrm{C}-\mathrm{C}=0.0049 \AA$

$b=14.1778(15) \AA$

$\beta=90^{\circ}$

$\alpha=90^{\circ}$

Calculated

3499.2(6) $\AA^{3}$

P 212121

P 2ac 2ab

C36 H44 O5 Si

584.80

1256.0

1256.87

$12,17,31$

7197[ 4038]
Wavelength $=0.71073 \AA$

$\mathrm{C}=24.837(2) \AA$

$\mathrm{Y}=90^{\circ}$

Reported

3499.2(6) $\AA^{3}$

P 212121

P 2ac $2 a b$

C36 H44 O5 Si

584.80

1.110

4

0.104

1256.0

$12,17,31$

7127

Theta $(\max )=26.413$

$w R 2$ (reflections) $=0.1118(7127)$

Npar $=390$

Compound 16a (CCDC: 2068621); The ellipsoid contour \% probability levels in the caption for the image of 16 a was $50 \%$.
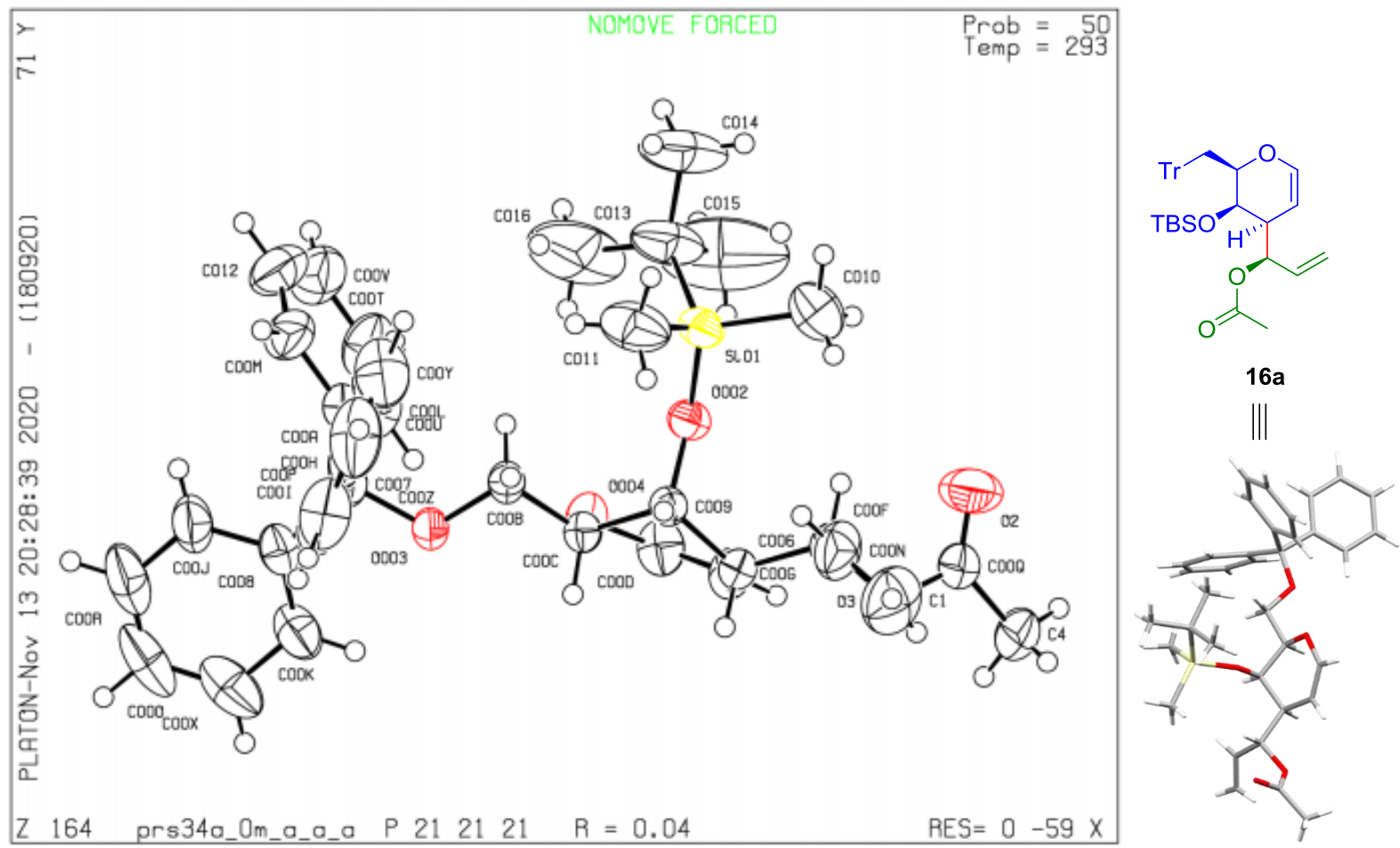
Crystal data and structure refinement for 21a: CCDC: 2068611

Crystal system

Crystal size

Bond precision:

Cell:

Temperature:

Volume

Space group

Hall group

Empirical formula

M.Wt

$\mathrm{Dx}, \mathrm{g} \mathrm{cm}^{-3}$

Z

$\mathrm{Mu}\left(\mathrm{mm}^{-1}\right)$

F000

F000'

$\mathrm{h}, \mathrm{k}, \mathrm{Imax}$

Nref

Data completeness $=1.40 / 0.73$

$\mathrm{R}($ reflections $)=0.0366$ (3452)

$S=1.057$
Monoclinic

$0.20 \times 0.15 \times 0.10$

$\mathrm{C}-\mathrm{C}=0.0061 \AA$

$a=6.6416(2) \AA \quad b=13.4490(4) \AA$

$\alpha=90^{\circ}$

$\beta=100.830(3)^{\circ}$

$298 \mathrm{~K}$

Calculated

1495.64(8) $\AA^{3}$

P 21

$\mathrm{P} 2 \mathrm{yb}$

C26 $446 \mathrm{O}_{4} \mathrm{Si} 2$

478.81

1.063

2

0.144

524.0

524.52

$7,16,20$

$5256[2750]$

Wavelength $=0.71073 \AA$

$\mathrm{c}=17.0478(5) \AA$

$\mathrm{Y}=90^{\circ}$

Reported

1495.64(8) $\AA^{3}$

P 1211

P 2yb

$\mathrm{C} 26 \mathrm{H} 46 \mathrm{O} 4 \mathrm{Si} 2$

191.52

1.063

5

0.144

524.0

$7,16,20$

3860

Theta $(\max )=25.010$

wR2 (reflections) $=0.0902(3860)$

Npar $=301$

Compound 21a (CCDC: 2068611); The ellipsoid contour \% probability levels in the caption for the image of 21a was $50 \%$.

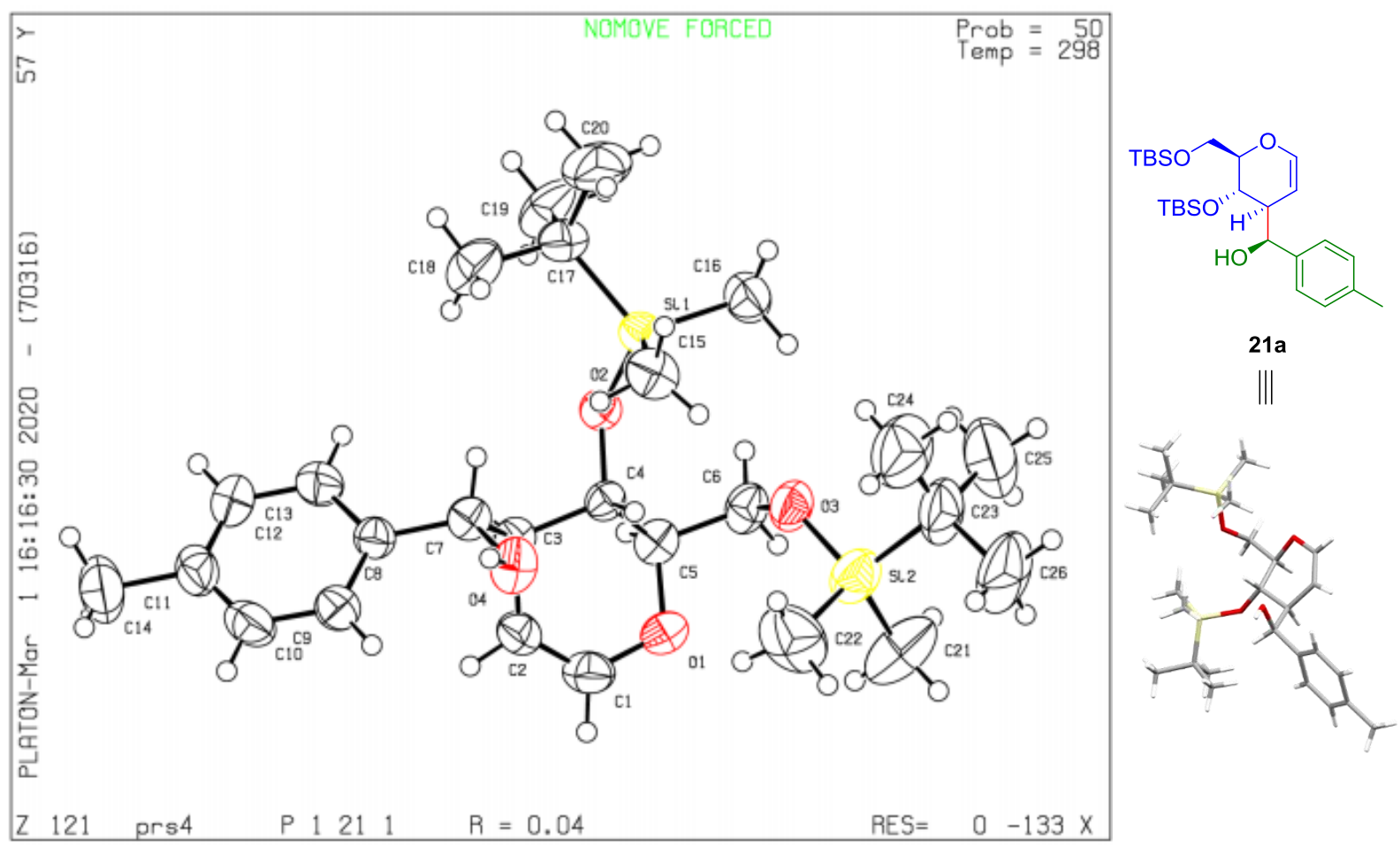


Crystal data and structure refinement for 23: CCDC: 2068613

Crystal system

Crystal size

Bond precision:

Cell:

Temperature:

Volume

Space group

Hall group

Empirical formula

M.Wt

$\mathrm{Dx}, \mathrm{g} \mathrm{cm}^{-3}$

Z

$\mathrm{Mu}\left(\mathrm{mm}^{-1}\right)$

F000

F000'

$\mathrm{h}, \mathrm{k}, \mathrm{Imax}$

Nref

Data completeness $=1.71 / 0.95$

$\mathrm{R}$ (reflections) $=0.0486(2985)$

$S=1.071$
Monoclinic

$0.20 \times 0.15 \times 0.10$

$\mathrm{C}-\mathrm{C}=0.0050 \AA$

$a=15.2600(5) \AA \quad b=5.3506(2) \AA$

$\alpha=90^{\circ}$

$\beta=91.847(3)^{\circ}$

$298 \mathrm{~K}$

Calculated

1792.44(11) $\AA^{3}$

12

$12 \mathrm{y}$

$\mathrm{C} 21 \mathrm{H} 22 \mathrm{O} 5$

354.39

1.313

4

0.093

752.0

752.41

$19,6,28$

$3926[2178]$
Wavelength $=0.71073 \AA$

$\mathrm{c}=21.9641(7) \AA$

$\mathrm{Y}=90^{\circ}$

Reported 1792.44(11) $\AA^{3}$

I 121

$12 \mathrm{y}$

$\mathrm{C} 21 \mathrm{H} 22 \mathrm{O} 5$

354.38

1.313

4

0.093

752.0

$19,6,27$

3723

Theta $(\max )=27.031$

wR2 $($ reflections $)=0.1610(3723)$

Npar $=237$

Compound 23 (CCDC: 2068613); The ellipsoid contour \% probability levels in the caption for the image of $\mathbf{2 3}$ was $50 \%$.

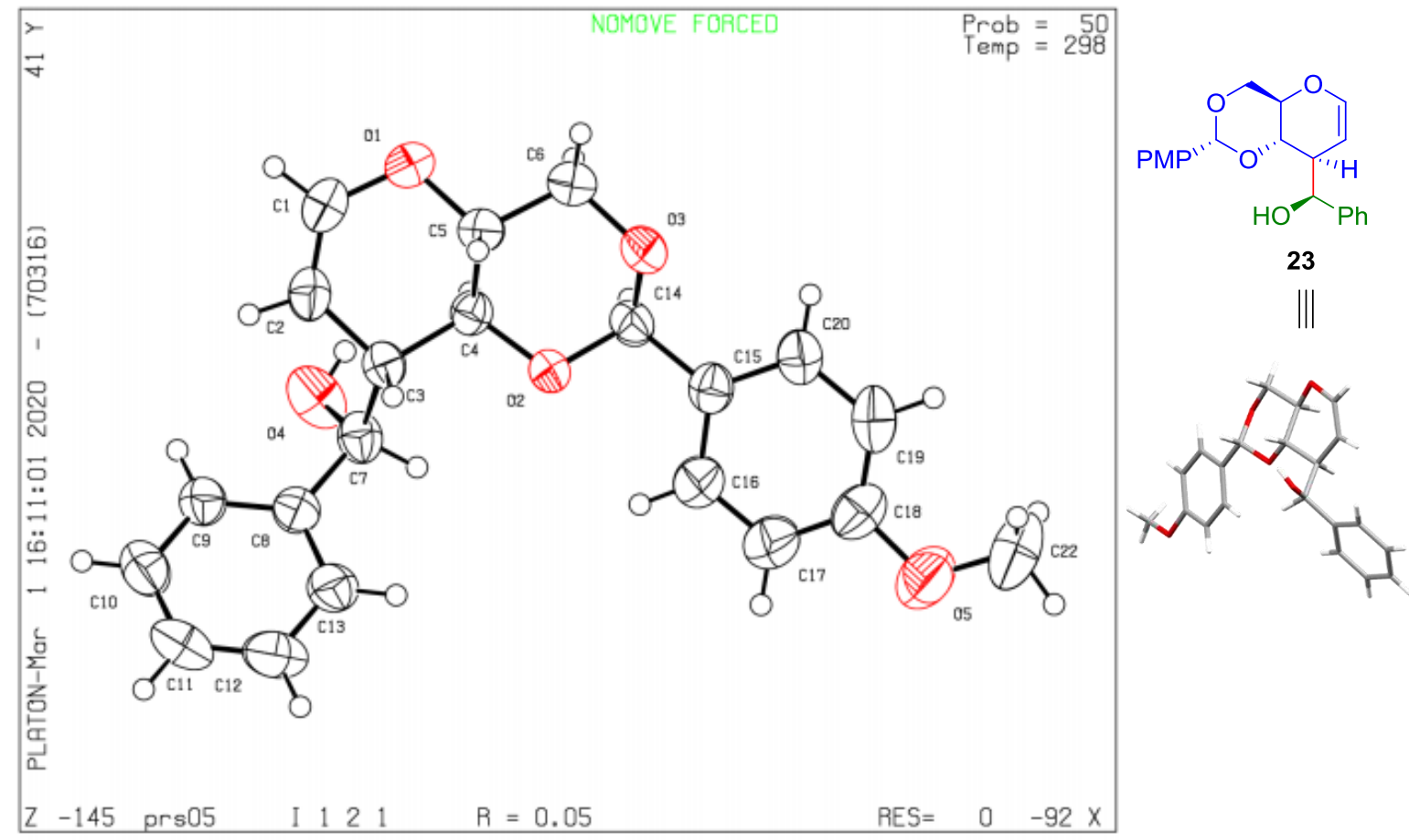


Crystal data and structure refinement for 25: CCDC: 2068620

Crystal system

Crystal size

Bond precision:

Cell:

Temperature:

Volume

Space group

Hall group

Empirical formula

M.Wt

$\mathrm{Dx}, \mathrm{g} \mathrm{cm}^{-3}$

Z

$\mathrm{Mu}\left(\mathrm{mm}^{-1}\right)$

F000

F000'

h,k,Imax

Nref

Data completeness $=1.87 / 0.99$

$\mathrm{R}$ (reflections $)=0.0315$ ( 3138)

$S=1.092$

$293 \mathrm{~K}$

P 21

$\mathrm{P} 2 \mathrm{yb}$

1.280

2

0.090

404.0
Monoclinic

$0.20 \times 0.15 \times 0.12$

$\mathrm{C}-\mathrm{C}=0.0038 \AA$

$\mathrm{b}=10.9797(8) \AA$

Wavelength $=0.71073 \AA$

$\mathrm{a}=4.7904(3) \AA$

$\beta=96.351(2)^{\circ}$

$c=18.8779(14) \AA$

$Y=90^{\circ}$

Calculated

986.83(12) $\AA^{3}$

$\mathrm{C} 23 \mathrm{H} 24 \mathrm{O} 5$

380.42

404.21

$5,13,22$

$3493[1846]$
Reported 986.83(12) $\AA^{3}$

P 1211

$\mathrm{P} 2 \mathrm{yb}$

$\mathrm{C} 23 \mathrm{H} 24 \mathrm{O} 5$

380.42

1.280

2

0.090

404.0

$5,13,22$

3457

Compound 25 (CCDC: 2068620 ); The ellipsoid contour \% probability levels in the caption for the image of $\mathbf{2 5}$ was $50 \%$.

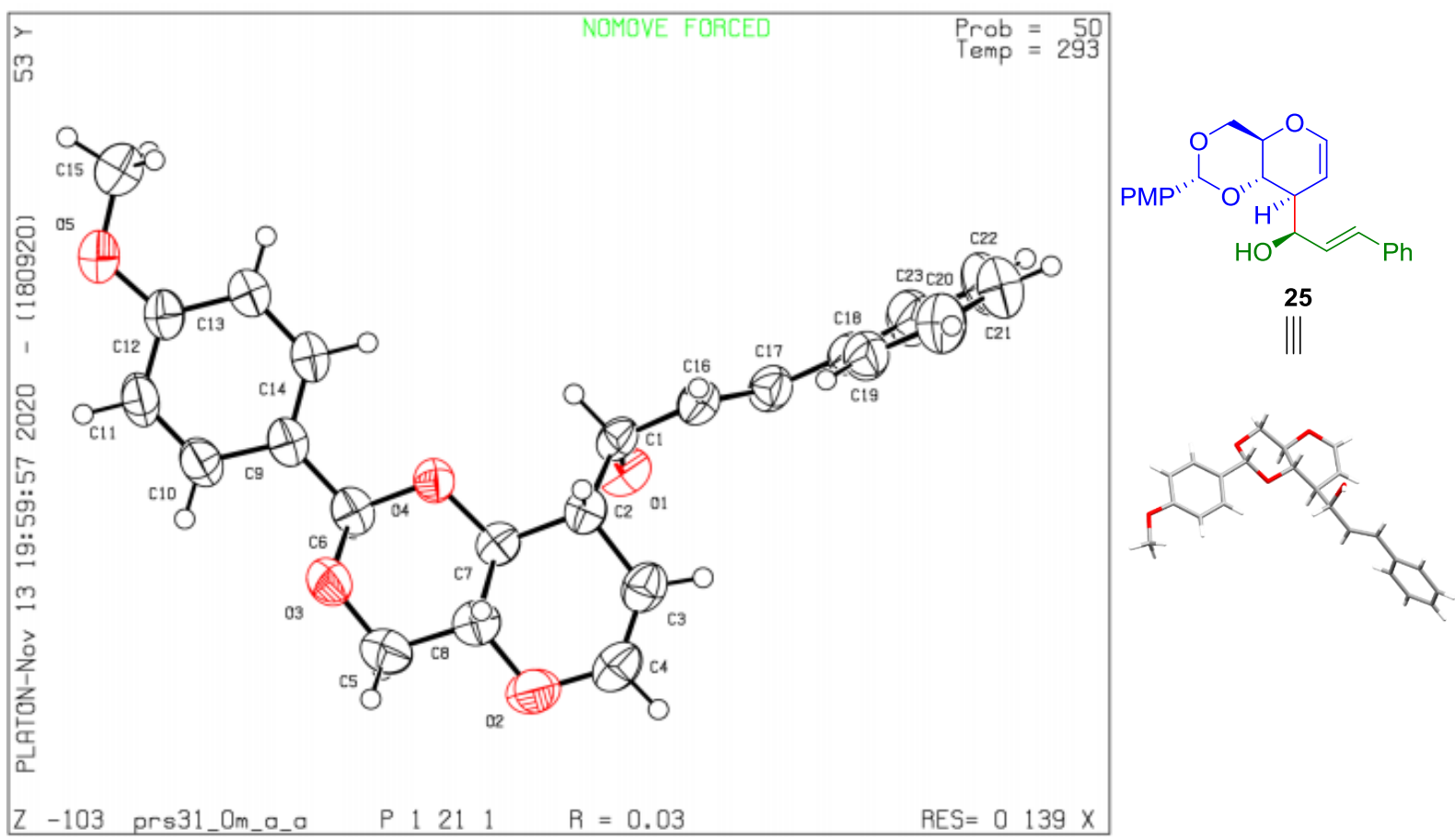


Crystal data and structure refinement for 27: CCDC: 2068616

Crystal system

Crystal size

Bond precision:

Cell:

Temperature:

Volume

Space group

Hall group

Empirical formula

M.Wt

$\mathrm{Dx}, \mathrm{g} \mathrm{cm}^{-3}$

Z

$\mathrm{Mu}\left(\mathrm{mm}^{-1}\right)$

F000

F000'

$\mathrm{h}, \mathrm{k}, \mathrm{Imax}$

Nref

Data completeness $=1.52 / 0.85$

$\mathrm{R}$ (reflections $)=0.0934(1212)$

$S=0.930$

P 21

$\mathrm{P} 2 \mathrm{yb}$

1.275

2

0.087

372.0
Monoclinic

$0.30 \times 0.20 \times 0.10$

$\mathrm{C}-\mathrm{C}=0.0170 \AA$

$$
\begin{aligned}
& b=4.6968(4) \AA \\
& \beta=111.102(12)^{\circ}
\end{aligned}
$$$$
\text { Wavelength }=0.71073 \AA
$$$$
a=13.2101(15) \AA
$$$$
\alpha=90^{\circ}
$$$$
\mathrm{C}=15.7722(16) \AA
$$$$
\mathrm{Y}=90^{\circ}
$$

$301 \mathrm{~K}$

Calculated

912.97(18) $\AA^{3}$

$\mathrm{C} 22 \mathrm{H} 22 \mathrm{O} 4$

350.40

372.19

$16,5,20$

$3927[2212]$

Reported

912.97(17) $\AA^{3}$

P 1211

$\mathrm{P} 2 \mathrm{yb}$

$\mathrm{C} 22 \mathrm{H} 22 \mathrm{O} 4$

350.39

1.275

2

0.087

372.0

$16,5,19$

3355

Theta $(\max )=26.961$

$w R 2$ (reflections) $=0.2839(3355)$

Npar $=237$

Compound 27 (CCDC: 2068616); The ellipsoid contour \% probability levels in the caption for the image of $\mathbf{2 7}$ was $50 \%$.

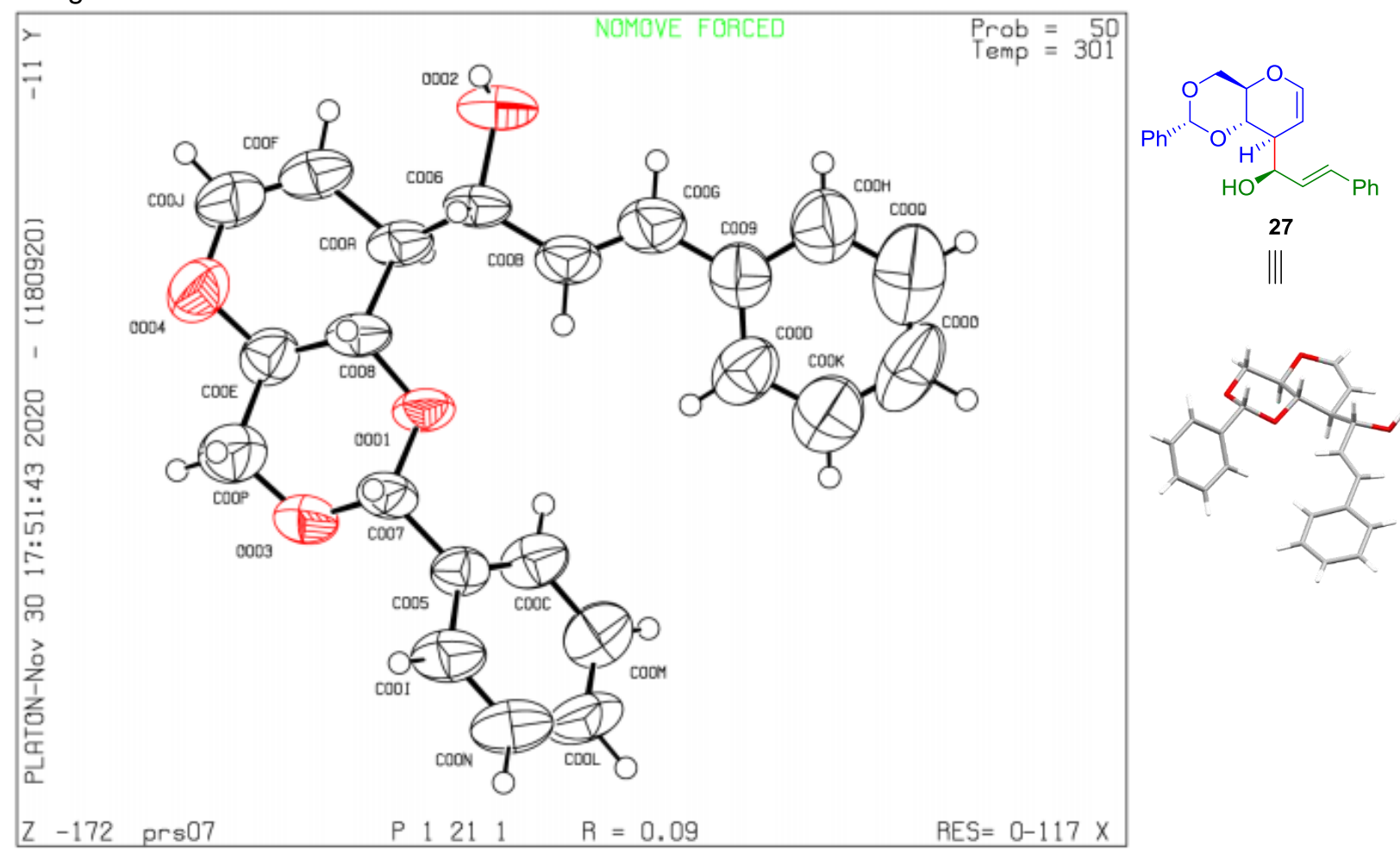


Crystal data and structure refinement for 31: CCDC: 2068625

Crystal system

Crystal size

Bond precision:

Cell:

Temperature:

Volume

Space group

Hall group

Empirical formula

M.Wt

$\mathrm{Dx}, \mathrm{g} \mathrm{cm}^{-3}$

Z

$\mathrm{Mu}\left(\mathrm{mm}^{-1}\right)$

F000

F000'

$\mathrm{h}, \mathrm{k}, \mathrm{Imax}$

Nref

Data completeness $=1.30 / 0.72$

$\mathrm{R}$ (reflections) $=0.0532(2012)$

$S=0.962$
Monoclinic

$0.20 \times 0.15 \times 0.10$

$\mathrm{C}-\mathrm{C}=0.0064 \AA$

$a=21.2635(13) \AA \mathrm{b}=5.1683(2) \AA$

$\alpha=90^{\circ}$

$\beta=110.995(7)^{\circ}$

$297 \mathrm{~K}$

Calculated

1934.0(2) $\AA^{3}$

C 2

$\mathrm{C} 2 \mathrm{y}$

$\mathrm{C} 23 \mathrm{H} 25 \mathrm{O} 5$

381.43

1.310

4

0.092

812.0

812.43

$26,6,24$

$4204[2341]$
Wavelength $=0.71073 \AA$

$c=18.8502(11) \AA$

$\mathrm{Y}=90^{\circ}$

Reported 1934.0(2) $\AA^{3}$

C 121

C $2 y$

$\mathrm{C} 23 \mathrm{H} 25 \mathrm{O} 5$

381.43

1.310

4

0.092

812.0

$26,6,23$

3040

Theta $(\max )=26.952$

$w R 2$ (reflections) $=0.1386(3040)$

Npar $=255$

Compound 31 (CCDC: 2068625); The ellipsoid contour \% probability levels in the caption for the image of $\mathbf{3 1}$ was $50 \%$.

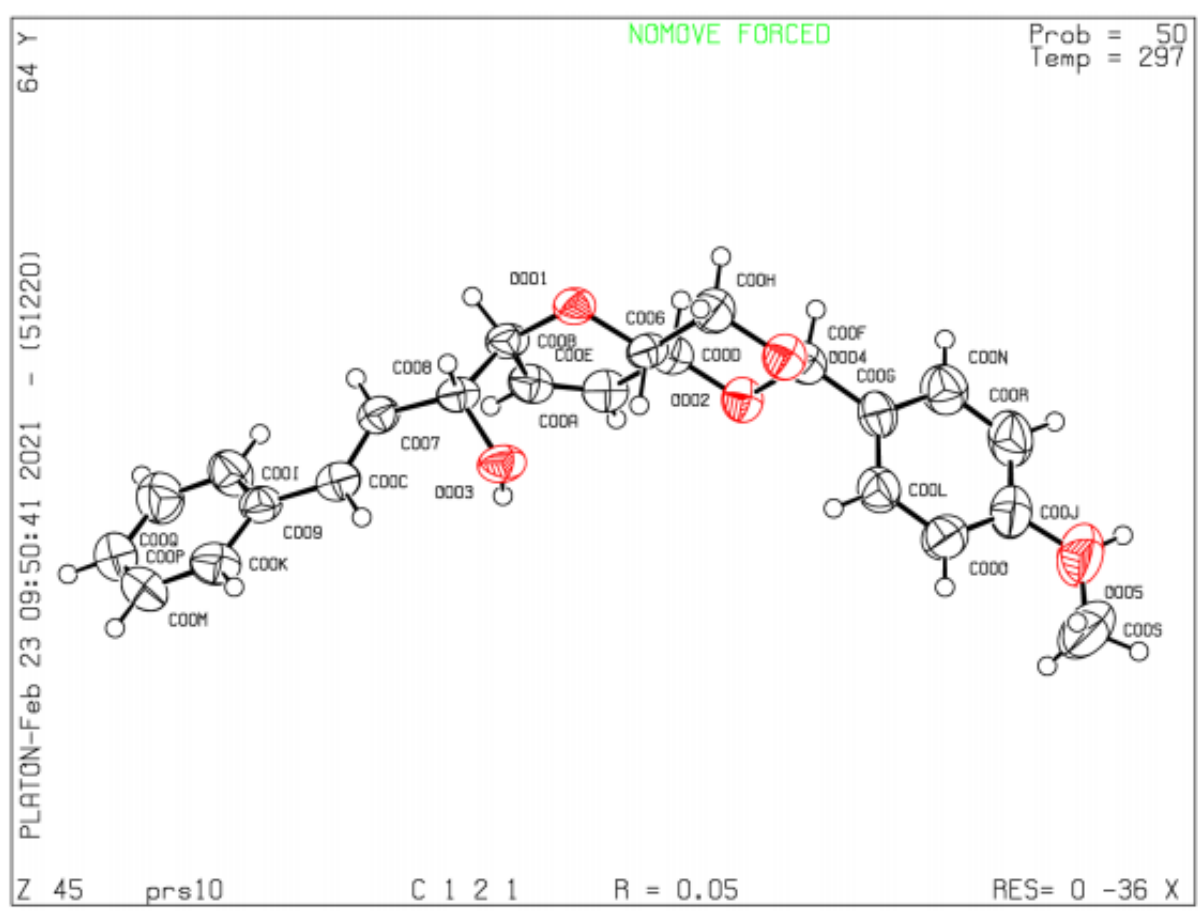

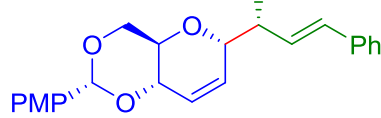

31

||

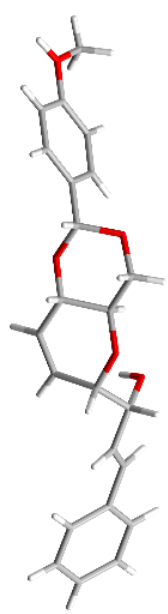


Crystal data and structure refinement for 32: CCDC: 2068618

Crystal system

Crystal size

Bond precision:

Cell:

Temperature:

Volume

Space group

Hall group

Empirical formula

M.Wt

$\mathrm{Dx}, \mathrm{g} \mathrm{cm}^{-3}$

Z

$\mathrm{Mu}\left(\mathrm{mm}^{-1}\right)$

F000

F000'

$\mathrm{h}, \mathrm{k}, \mathrm{Imax}$

Nref

Data completeness $=1.57 / 0.91$

$\mathrm{R}$ (reflections) $=0.0628(1553)$

$S=0.896$

$303 \mathrm{~K}$

4

0.409
Orthorhombic

$0.33 \times 0.21 \times 0.12$

$\mathrm{C}-\mathrm{C}=0.0136 \AA$

$\mathrm{a}=6.0292(5) \AA$

$b=13.4511(12) \AA$

Wavelength $=0.71073 \AA$

$\alpha=90^{\circ}$

$\beta=90^{\circ}$

$\mathrm{C}=29.979(2) \AA$

$\mathrm{Y}=90^{\circ}$

Calculated

2431.3(3) $\AA^{3}$

Reported

2431.3(3) $\AA^{3}$

P 212121

P 212121

P 2ac 2ab

C23 H24 O5, C D Cl3

500.79

$\mathrm{C} 23 \mathrm{H} 24 \mathrm{O} 5, \mathrm{C} D \mathrm{Cl} 3$

500.79

1.368

1040.0

1042.22

$7,17,38$

$5317[3070]$

$7,16,38$

4820

4

0.409

1040.0

Theta $(\max )=27.015$

wR2 $($ reflections $)=0.1592(4820)$

Npar $=292$

Compound 32 (CCDC: 2068618); The ellipsoid contour \% probability levels in the caption for the image of 32 was $50 \%$.
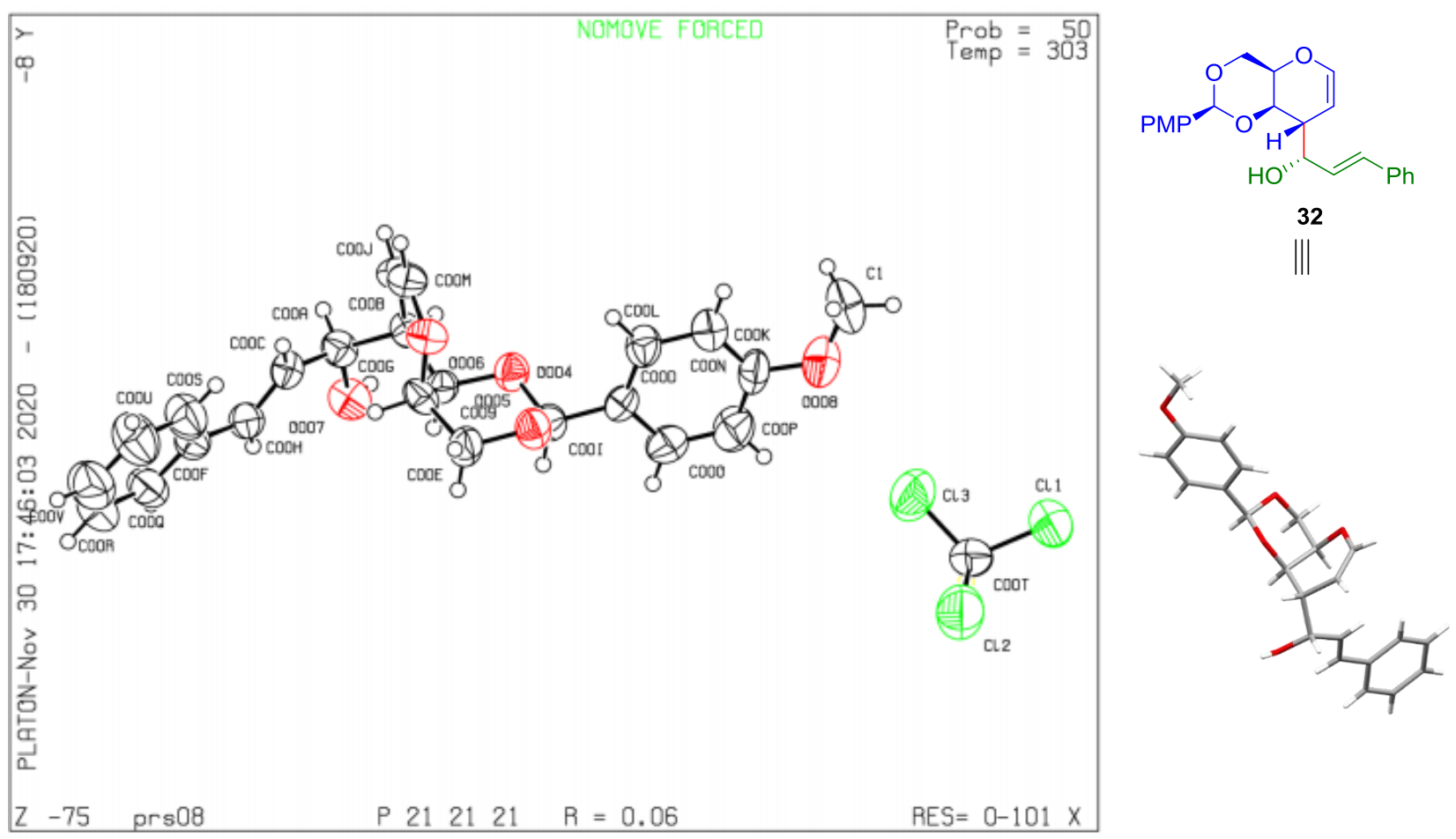


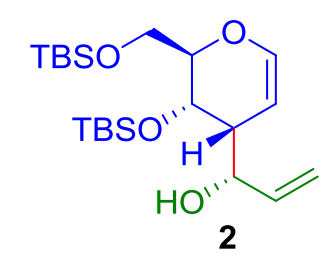

${ }^{1} \mathrm{H}$ NMR (400 MHz, $\mathrm{CDCl}_{3}$ )

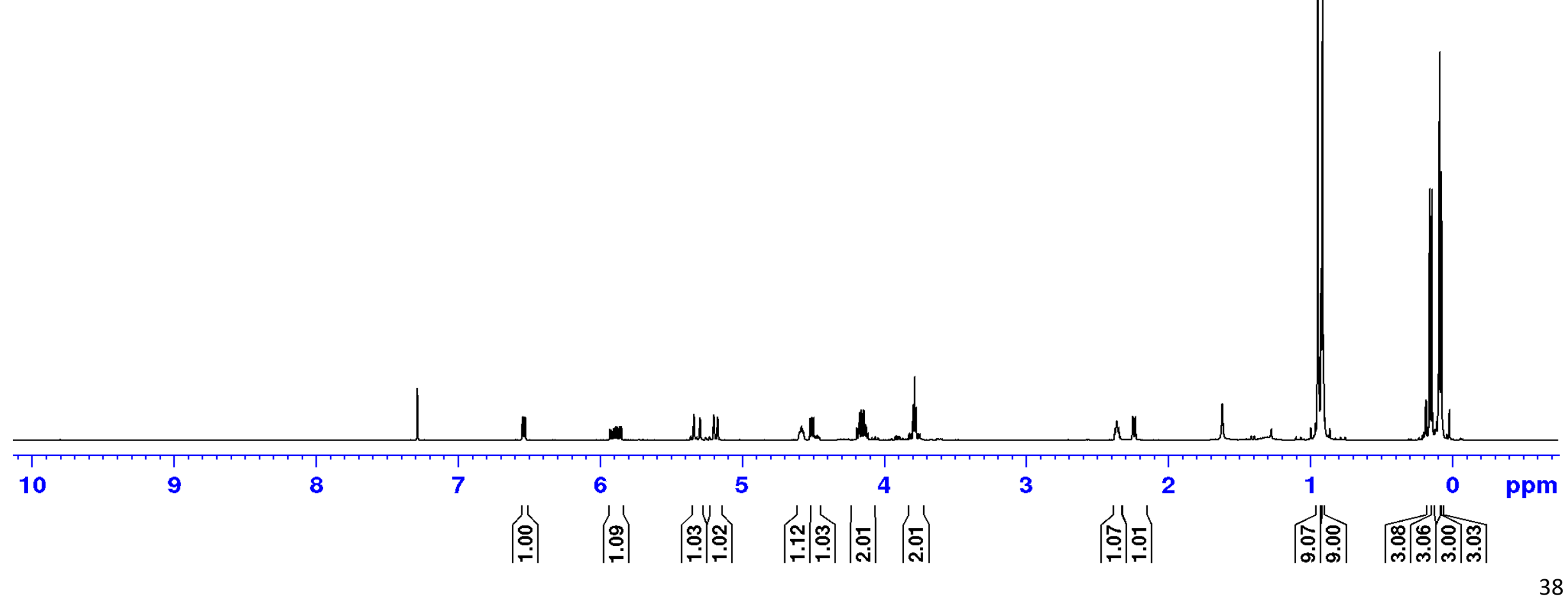




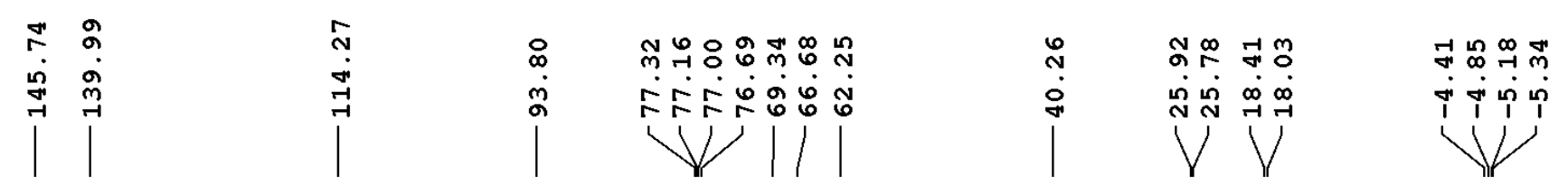

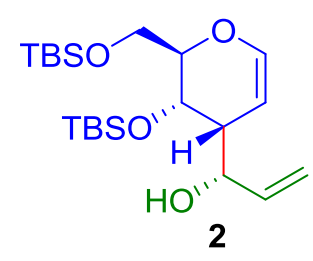

${ }^{13} \mathrm{C}$ NMR (100 MHz, $\mathrm{CDCl}_{3}$ )

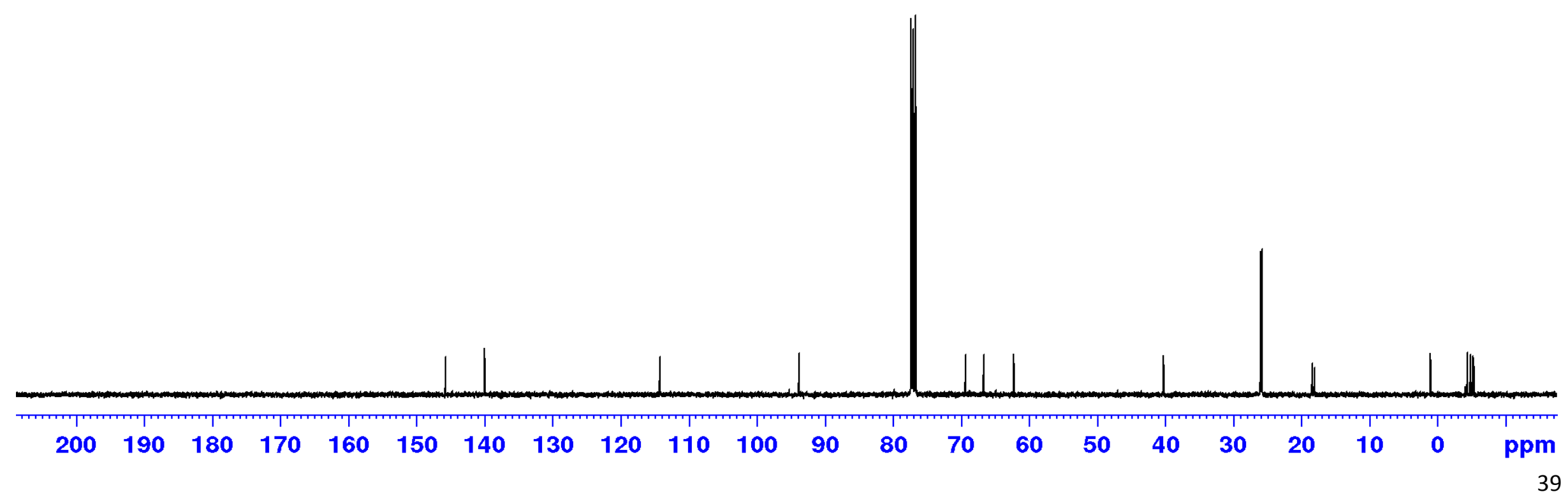




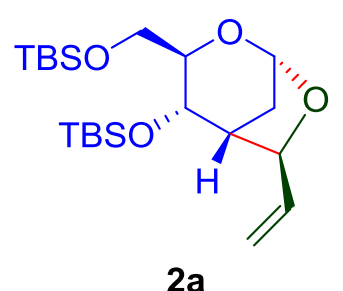

${ }^{1} \mathrm{H}$ NMR (500 MHz, $\mathrm{CDCl}_{3}$ )

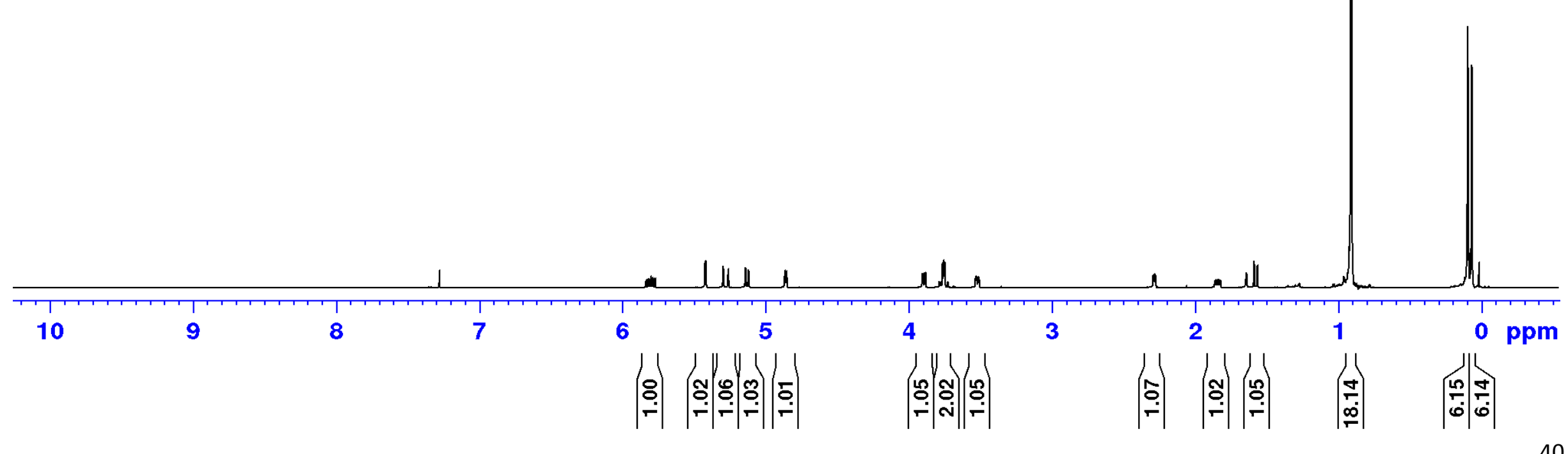



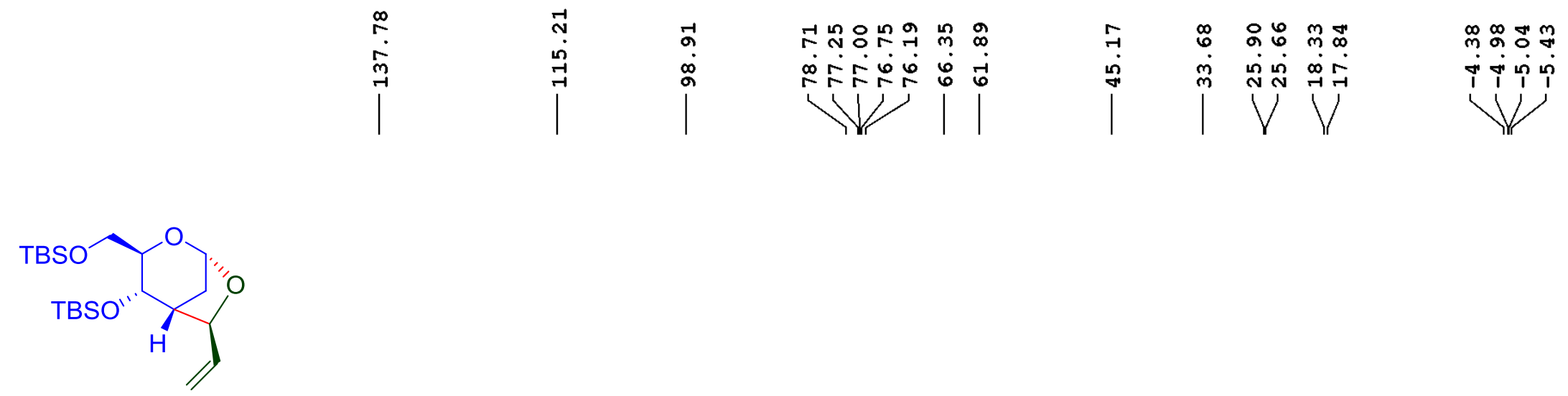

$2 a$

${ }^{13} \mathrm{C}$ NMR (125 MHz, $\mathrm{CDCl}_{3}$ )

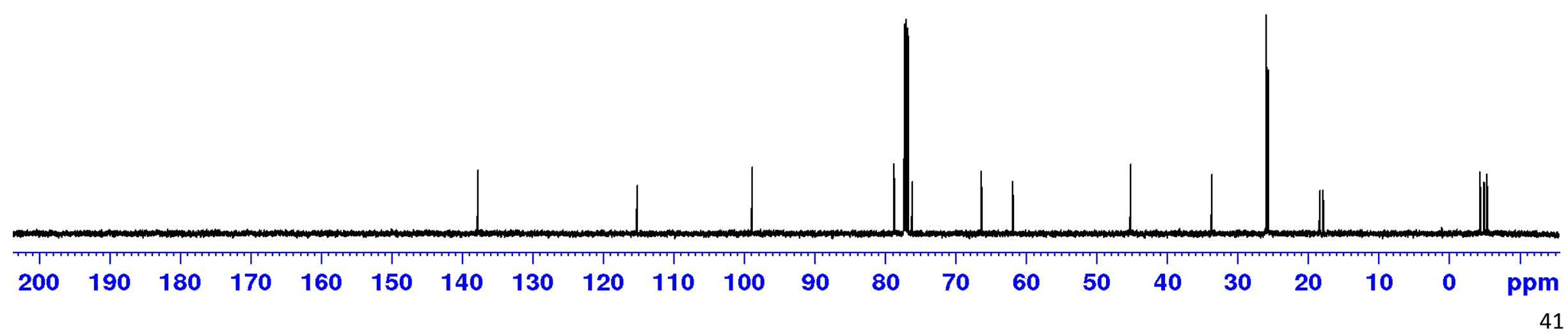




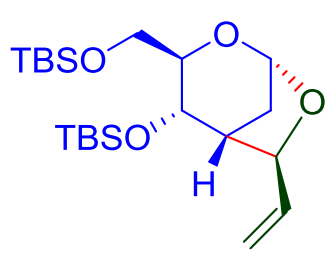

$2 a$

(COSY, $500 \mathrm{MHz}$ )

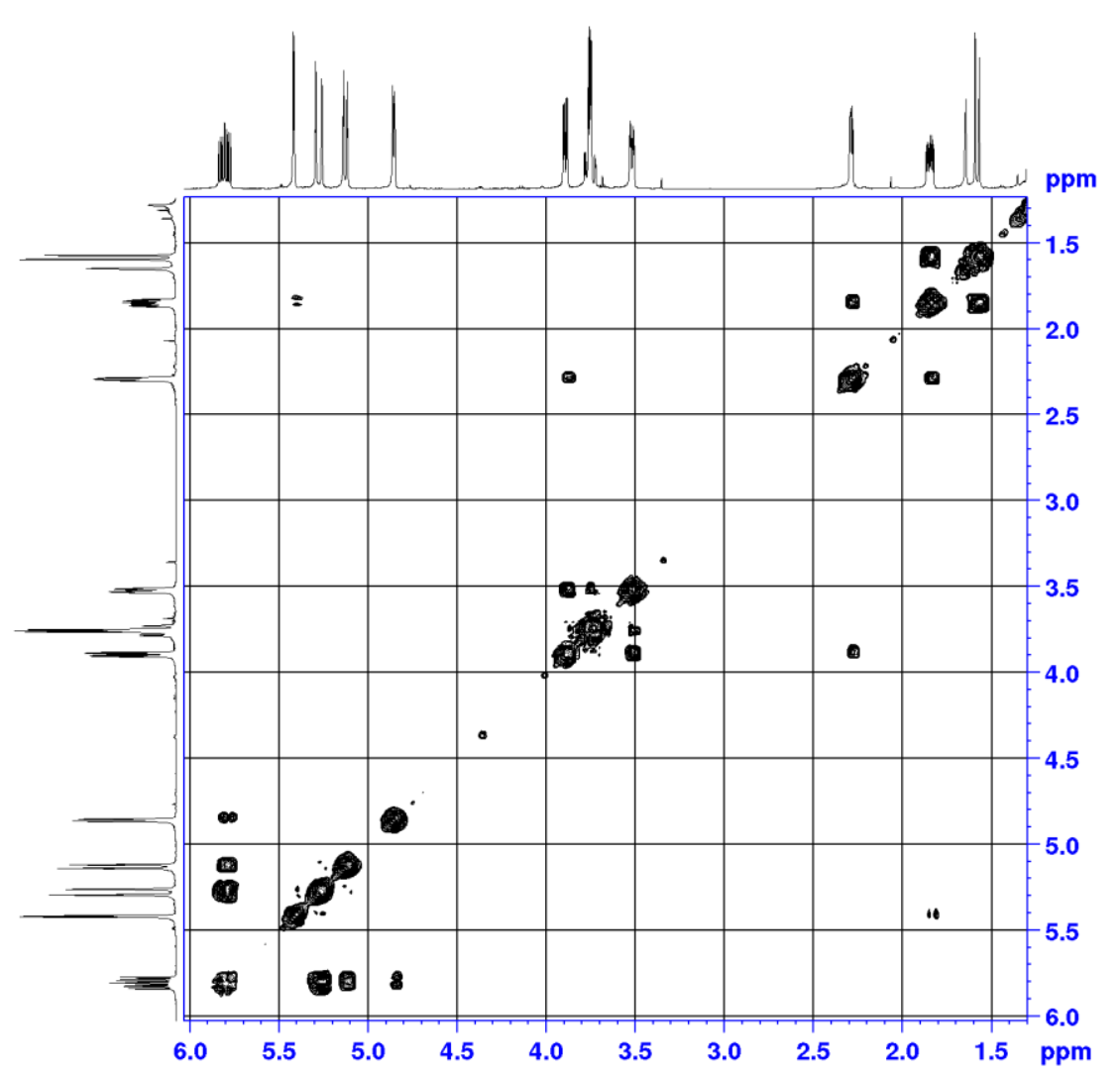

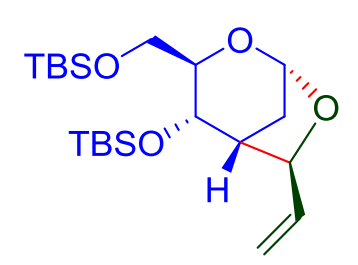

$2 a$

(NOESY, 500 MHz)

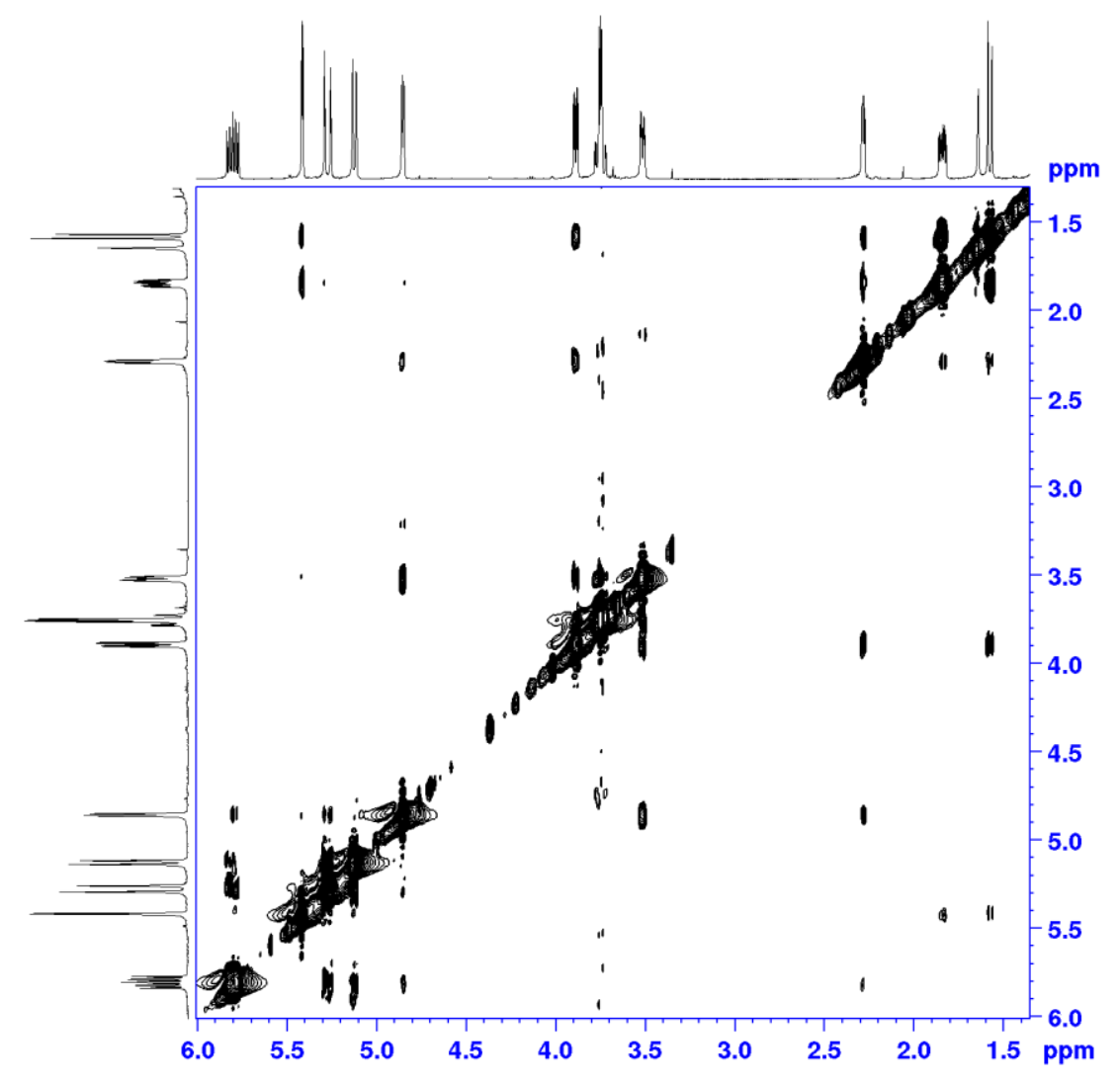




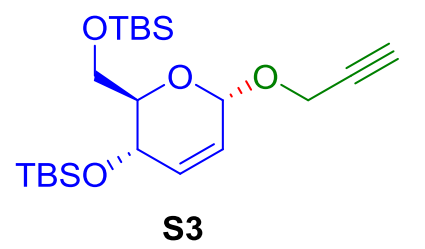

${ }^{1} \mathrm{H}$ NMR (500 MHz, $\mathrm{CDCl}_{3}$ )

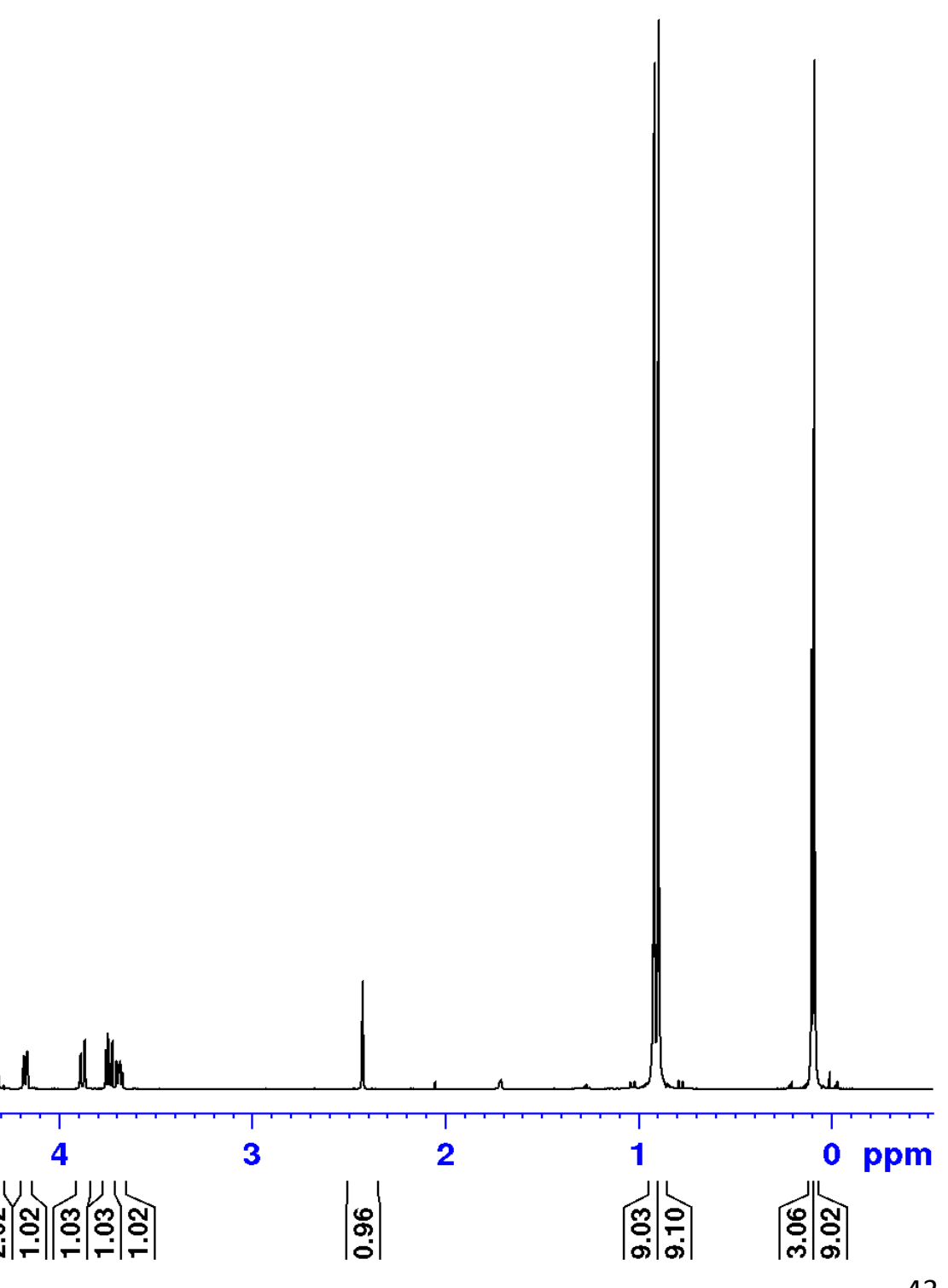


TBSO

OTBS

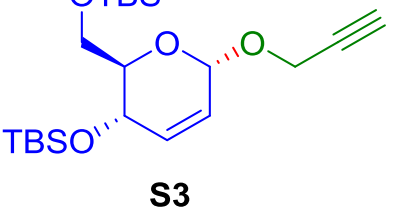

${ }^{13} \mathrm{C}$ NMR $\left(125 \mathrm{MHz}, \mathrm{CDCl}_{3}\right)$

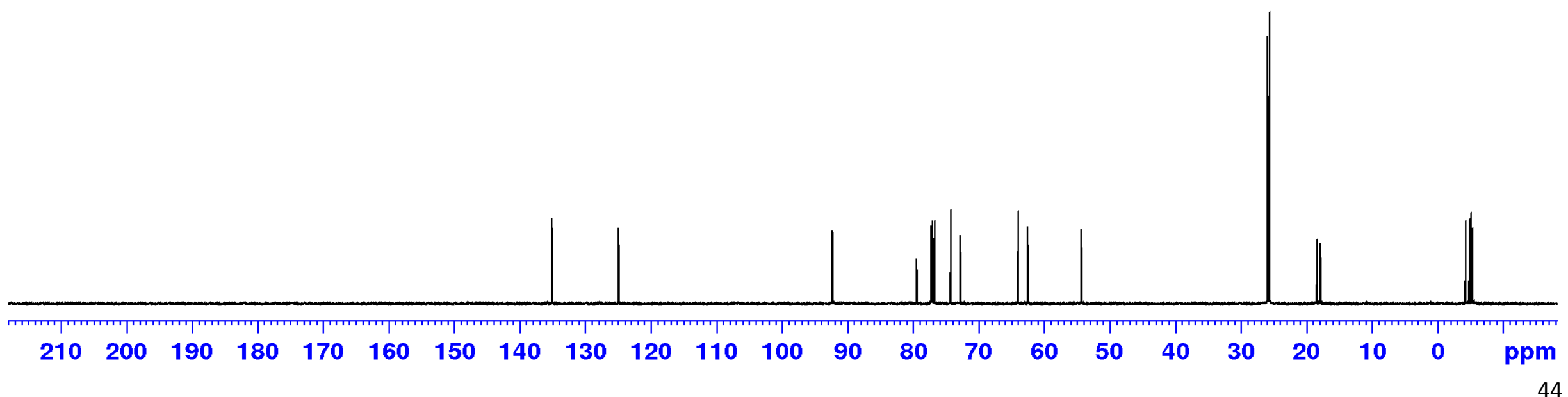




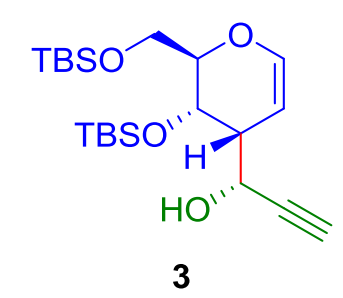

${ }^{1} \mathrm{H}$ NMR (500 MHz, $\mathrm{CDCl}_{3}$ )

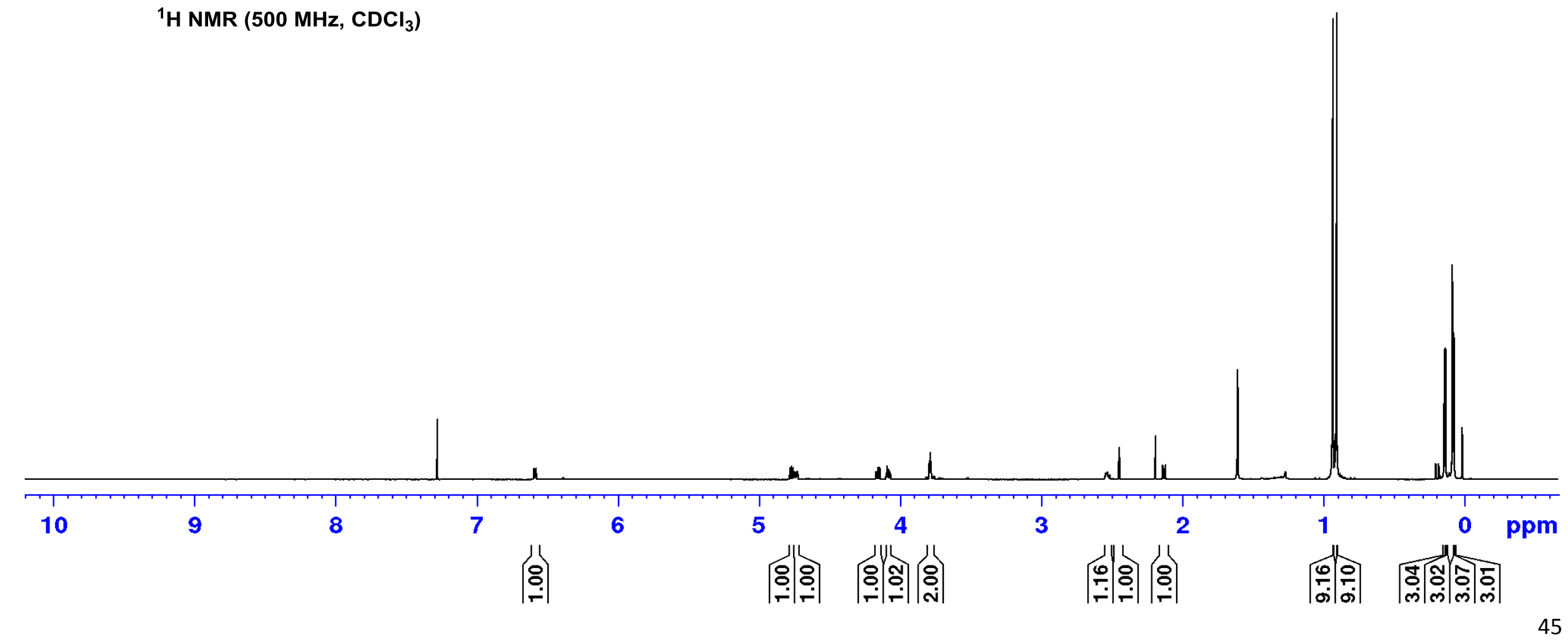



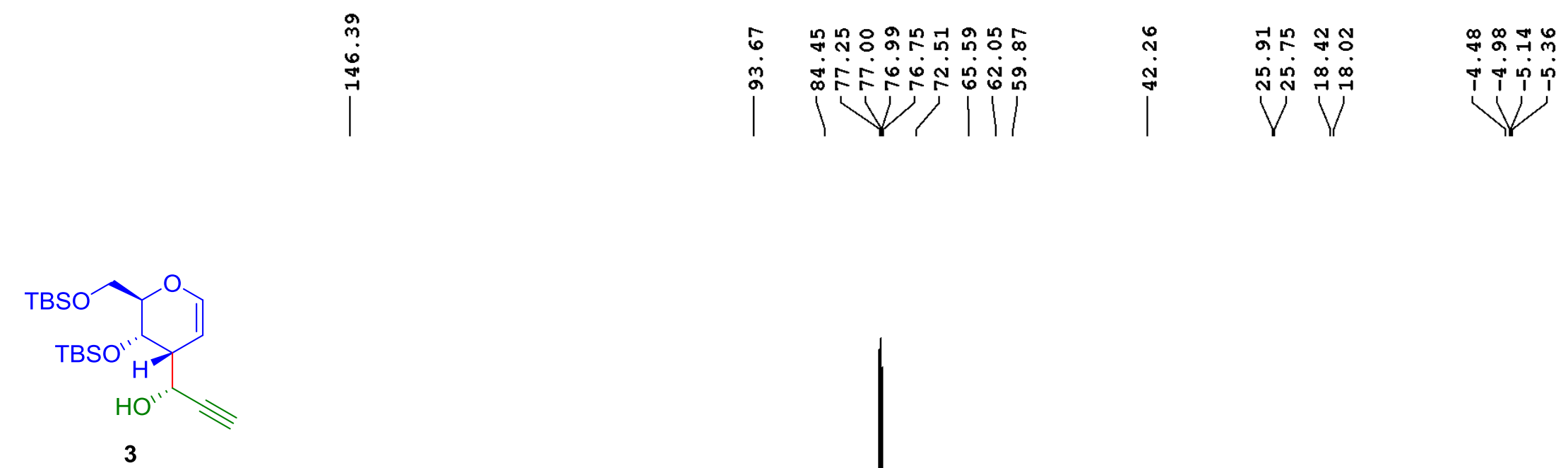

${ }^{13} \mathrm{C}$ NMR (125 MHz, $\left.\mathrm{CDCl}_{3}\right)$

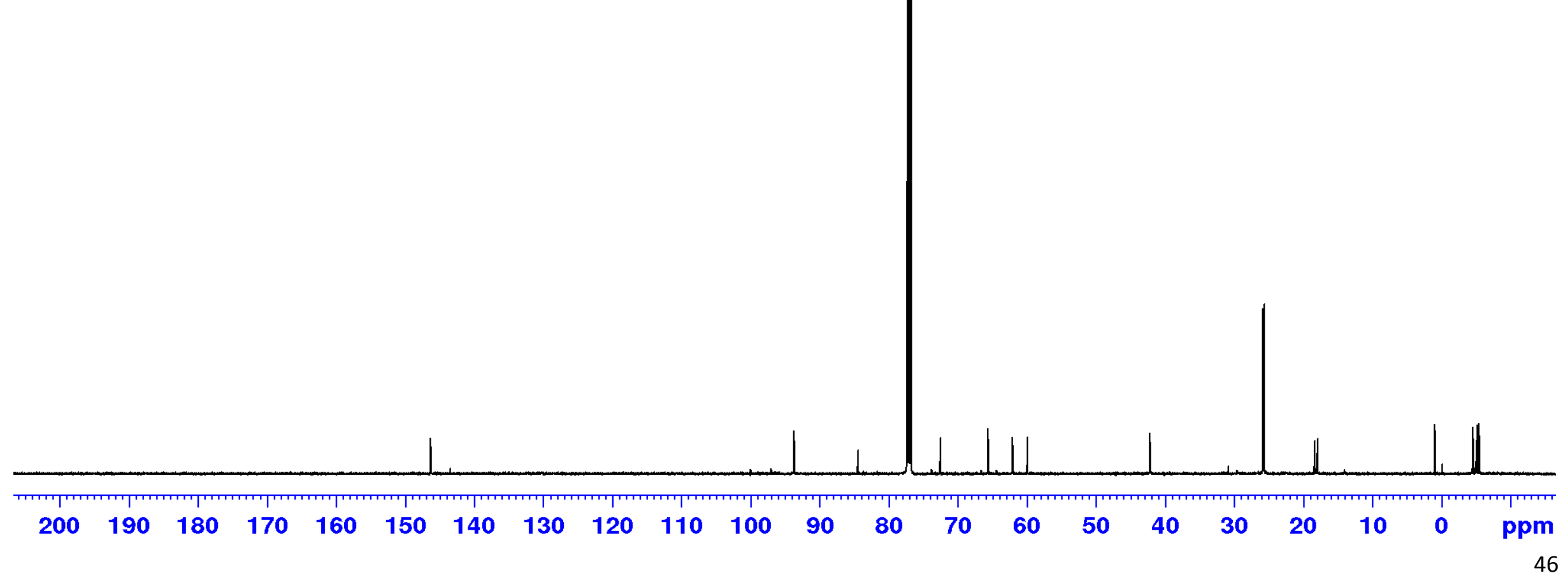




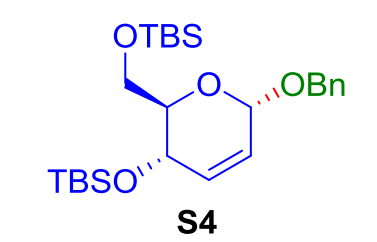

${ }^{1} \mathrm{H}$ NMR (500 MHz, $\mathrm{CDCl}_{3}$ )

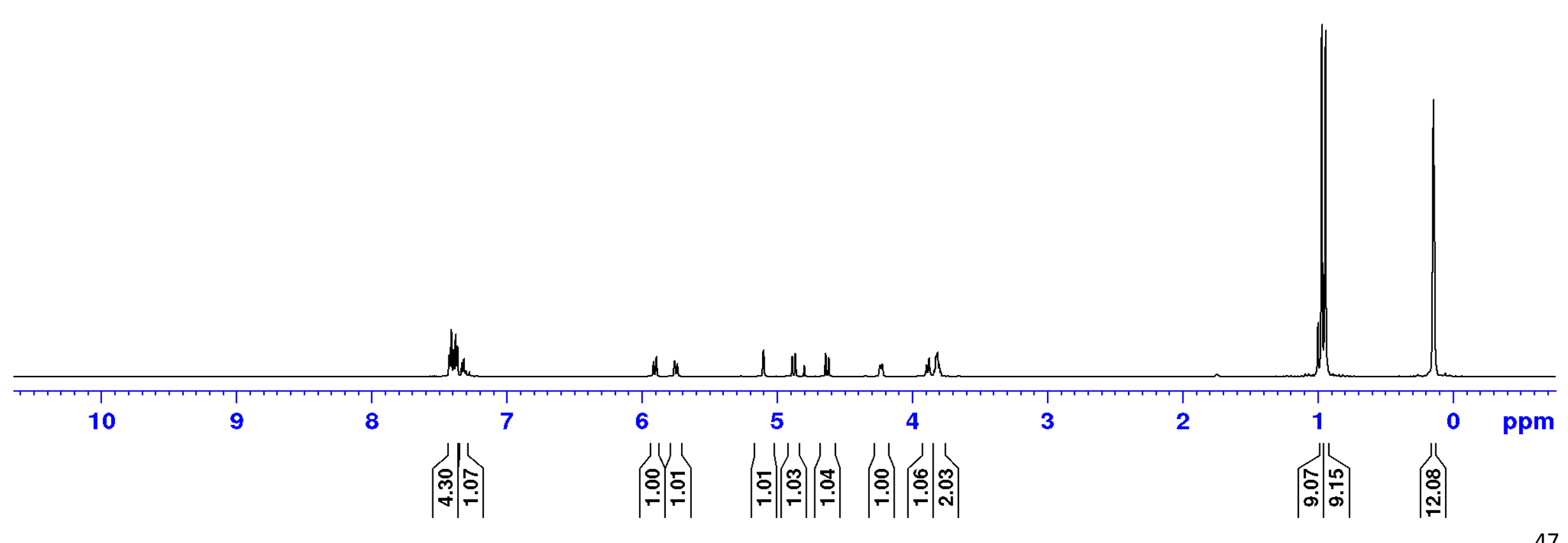



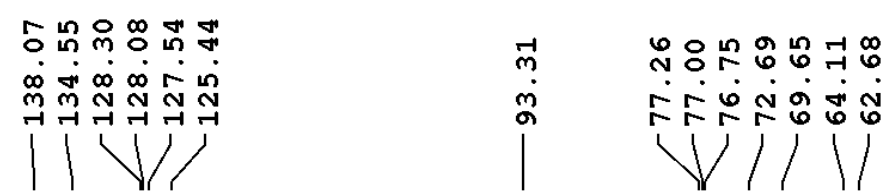

ธิ㇒日

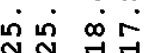

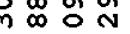

$\sqrt{N}$

गण फि

VIj।

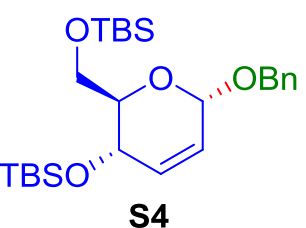

${ }^{13} \mathrm{C}$ NMR $\left(125 \mathrm{MHz}, \mathrm{CDCl}_{3}\right)$

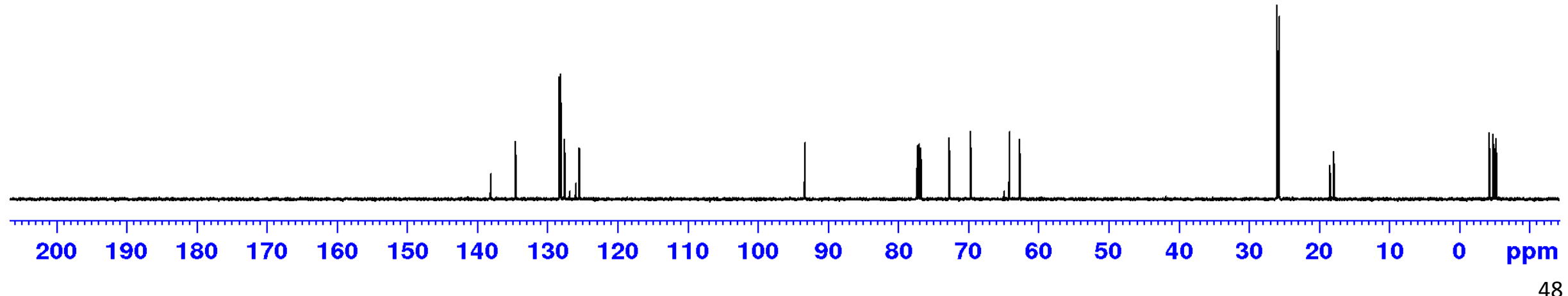




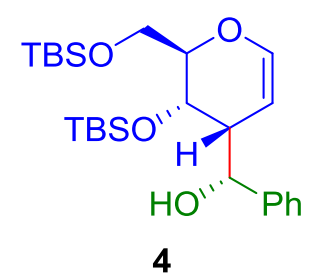

${ }^{1} \mathrm{H}$ NMR $\left(500 \mathrm{MHz}, \mathrm{CDCl}_{3}\right)$

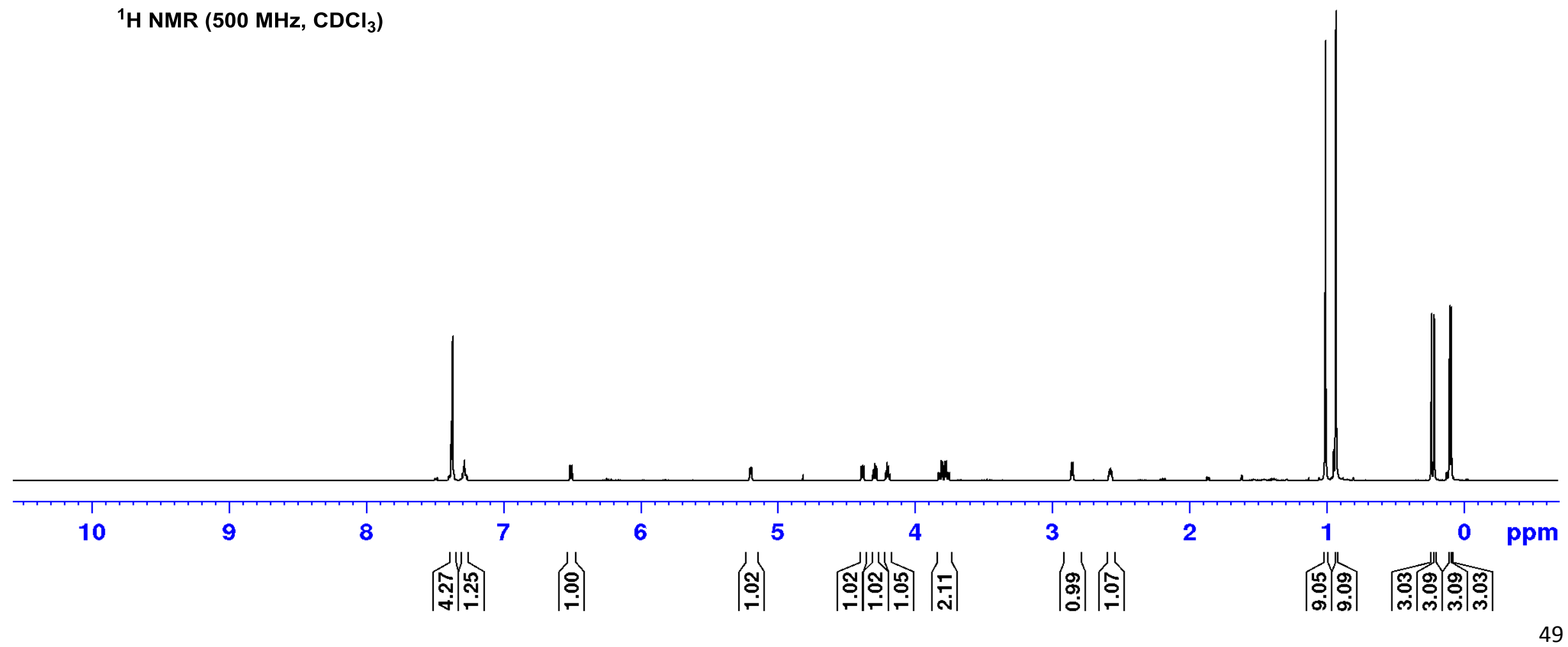




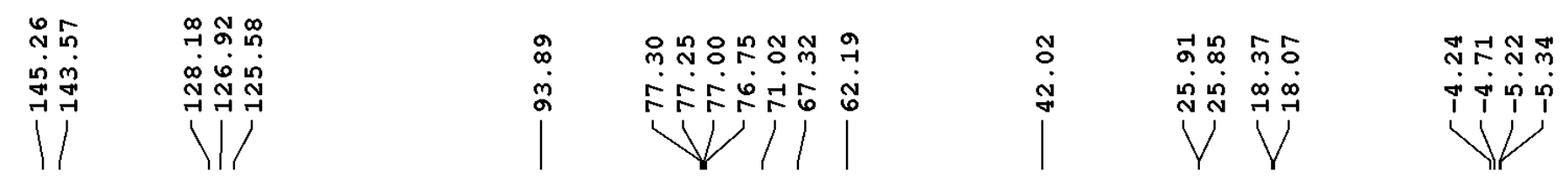

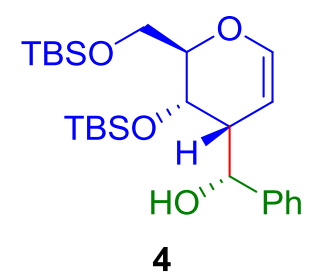

${ }^{13} \mathrm{C}$ NMR (125 MHz, $\mathrm{CDCl}_{3}$ )

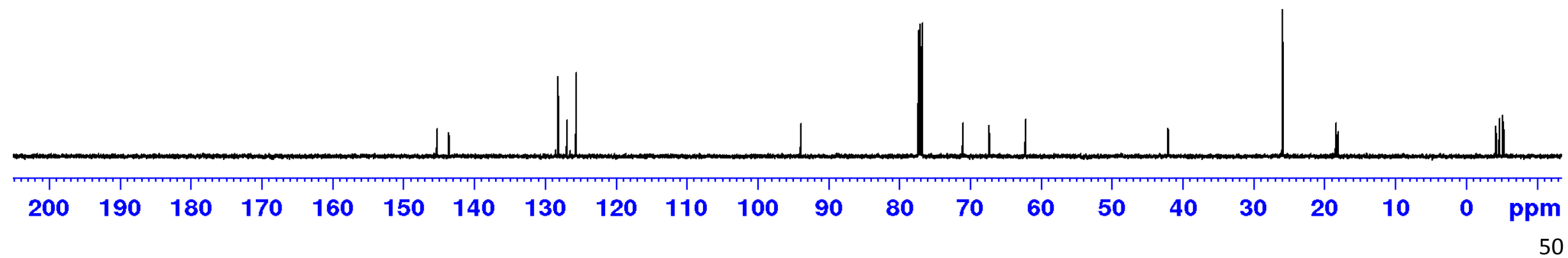




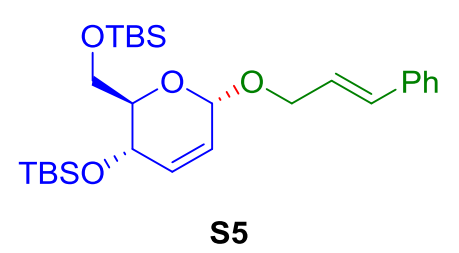

${ }^{1} \mathrm{H}$ NMR (500 MHz, $\mathrm{CDCl}_{3}$ )

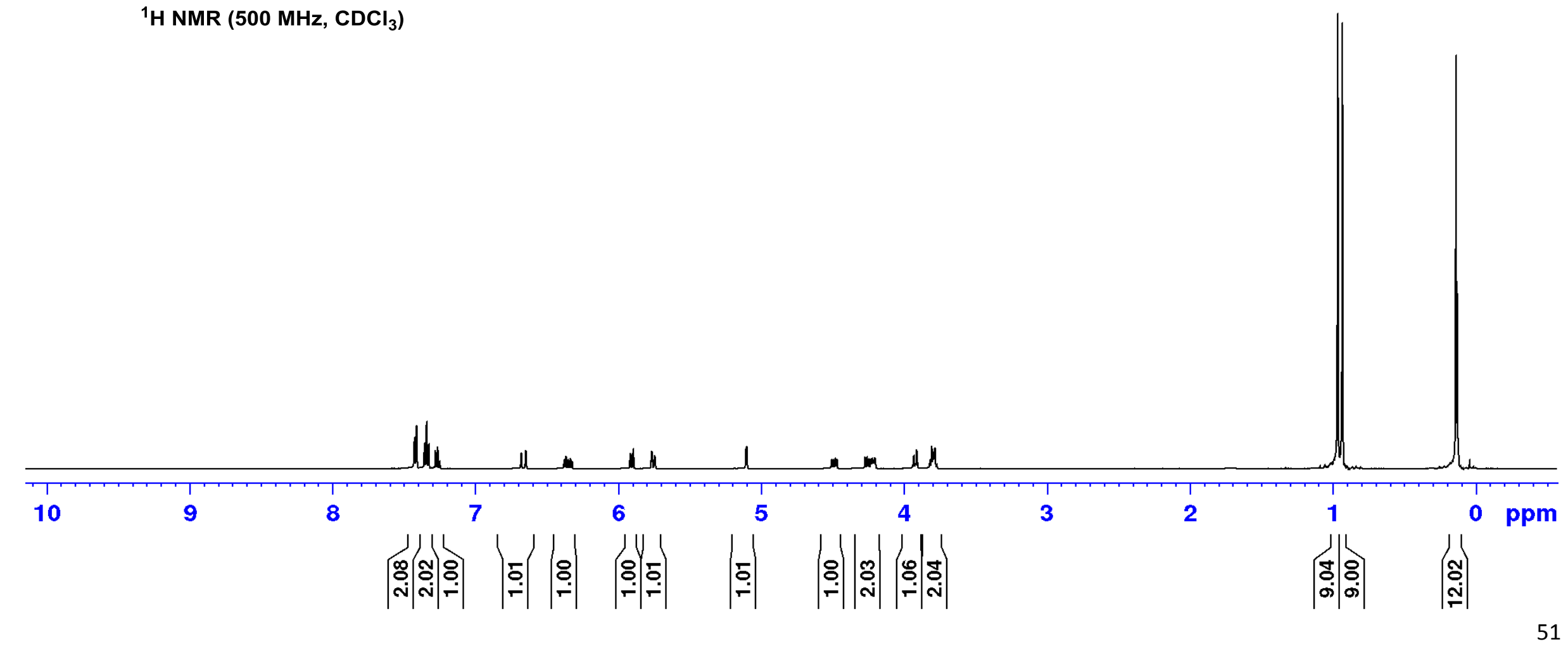




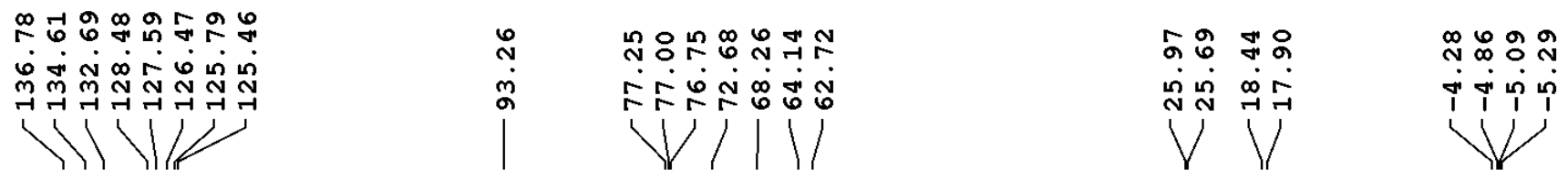

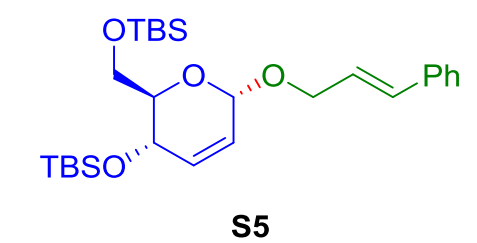

${ }^{13} \mathrm{C}$ NMR (125 MHz, $\mathrm{CDCl}_{3}$ )

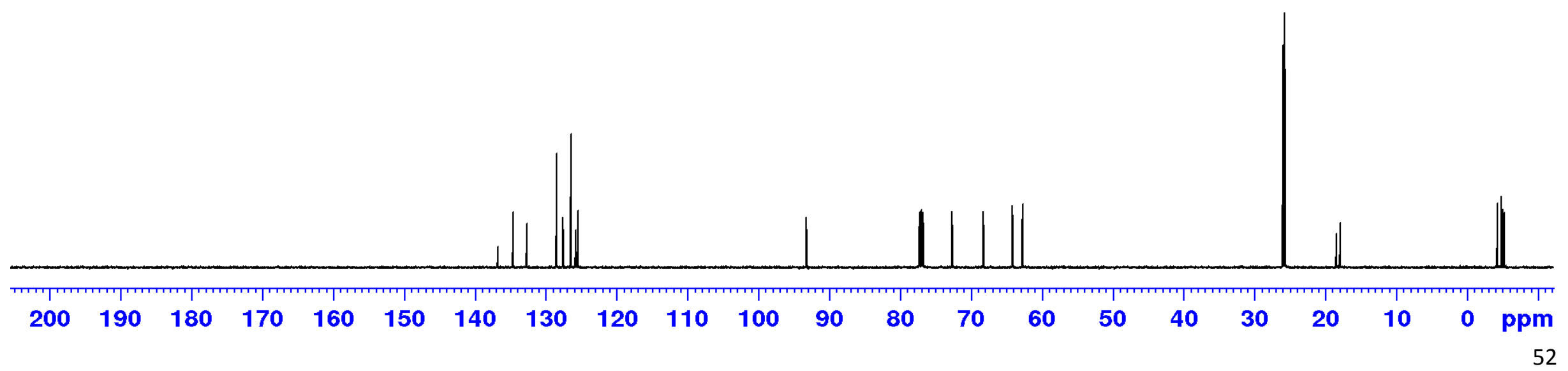




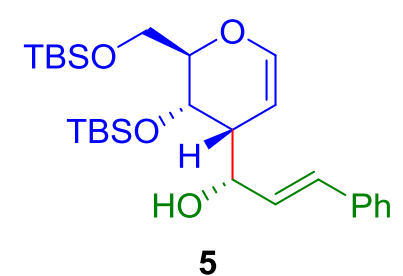

${ }^{1} \mathrm{H}$ NMR (500 $\mathrm{MHz} \mathrm{CDCl}_{3}$ )

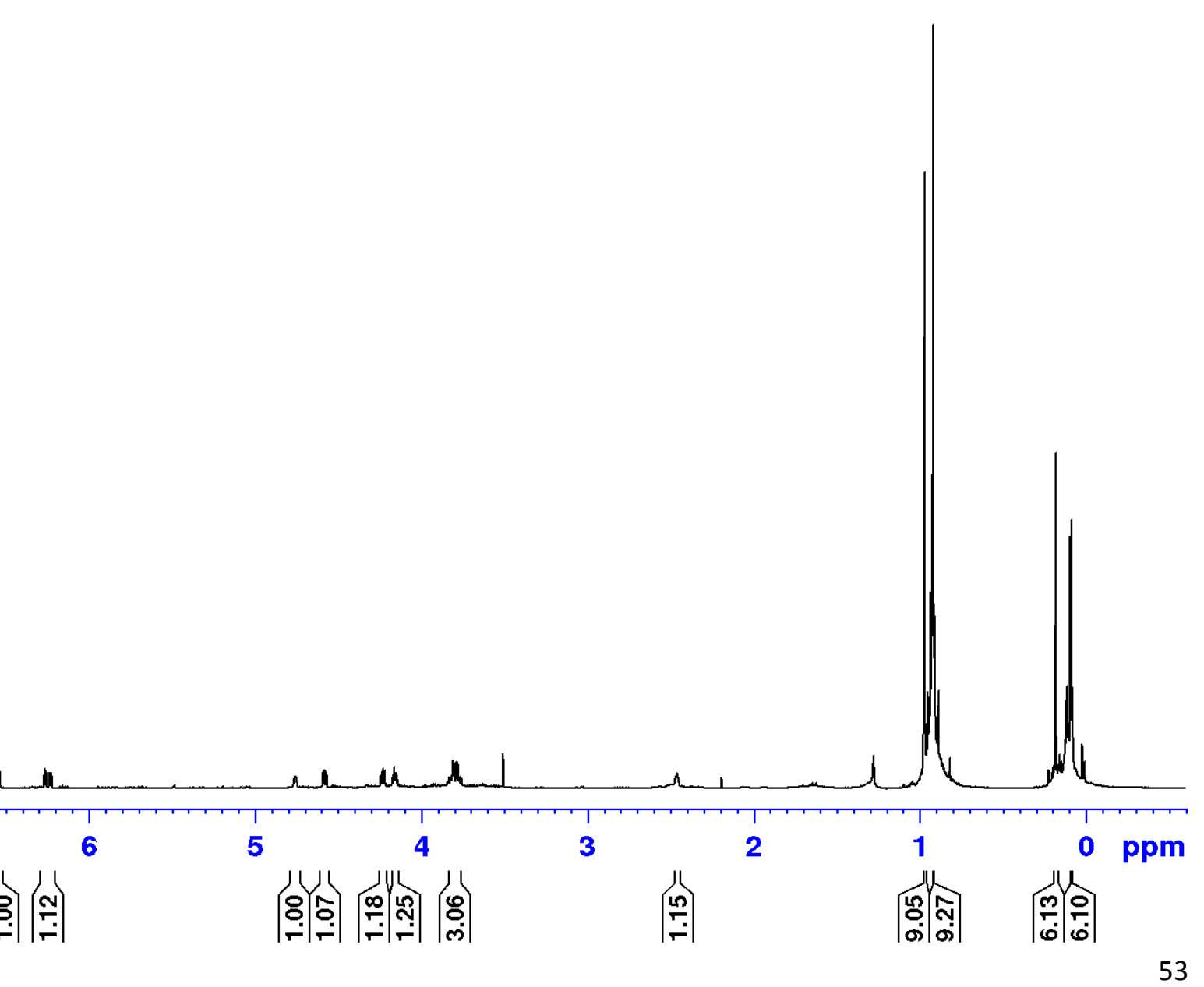



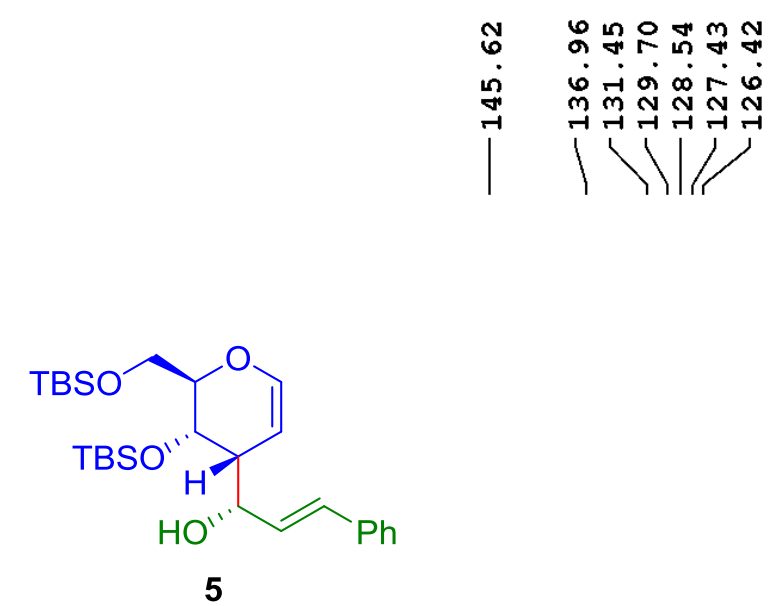

${ }^{13} \mathrm{C}$ NMR (125 MHz, $\mathrm{CDCl}_{3}$ )
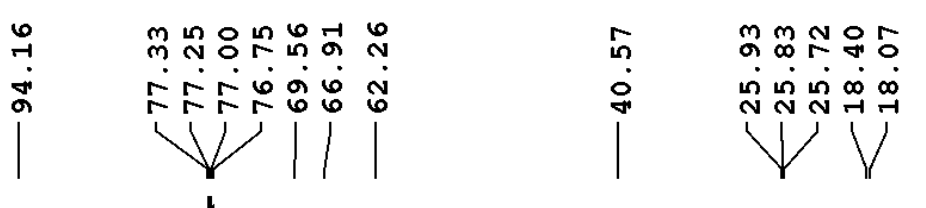

mํำ

i pi pi
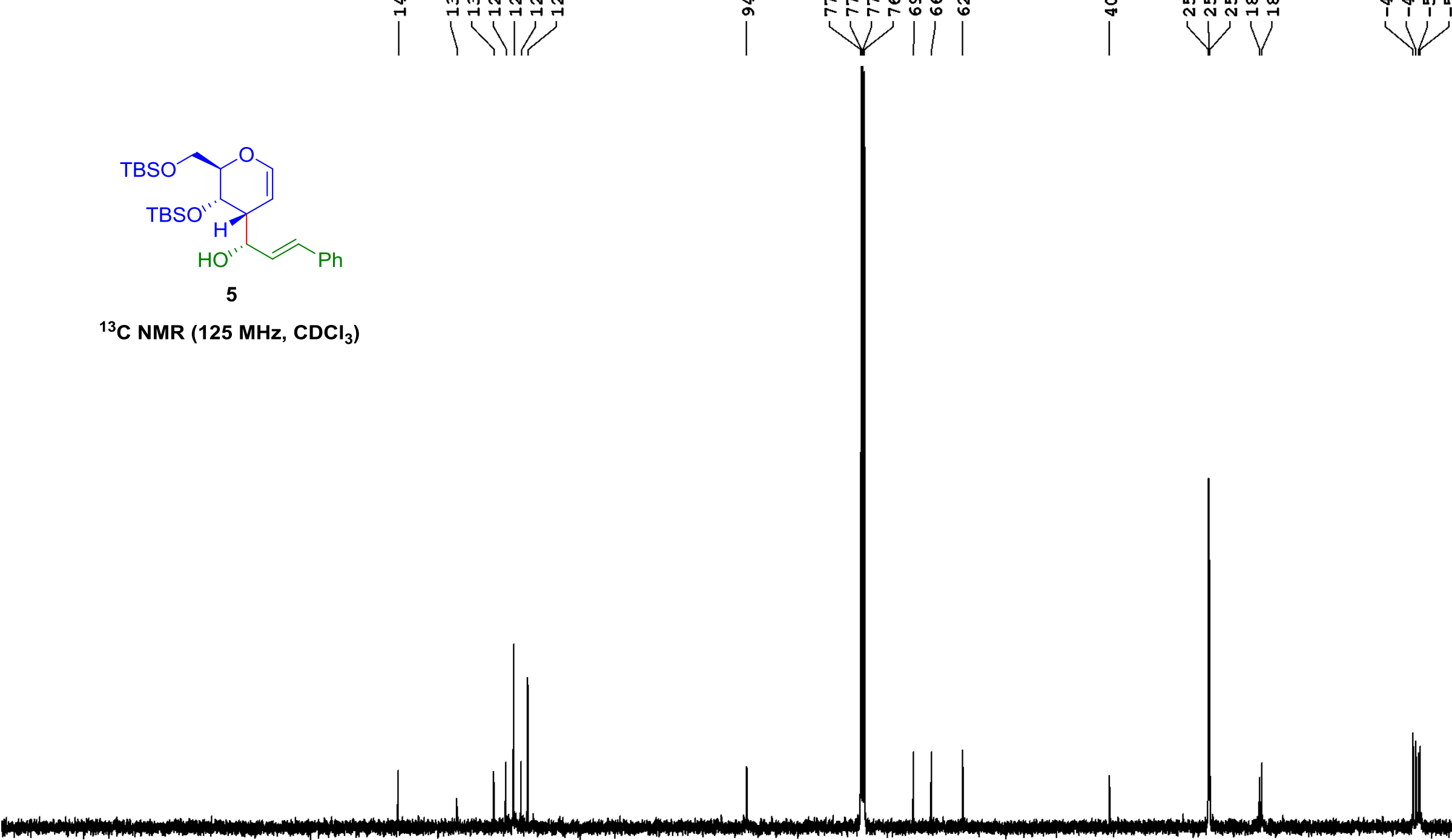


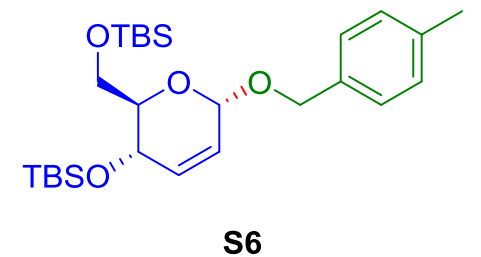

${ }^{1} \mathrm{H}$ NMR (500 MHz, $\mathrm{CDCl}_{3}$ )

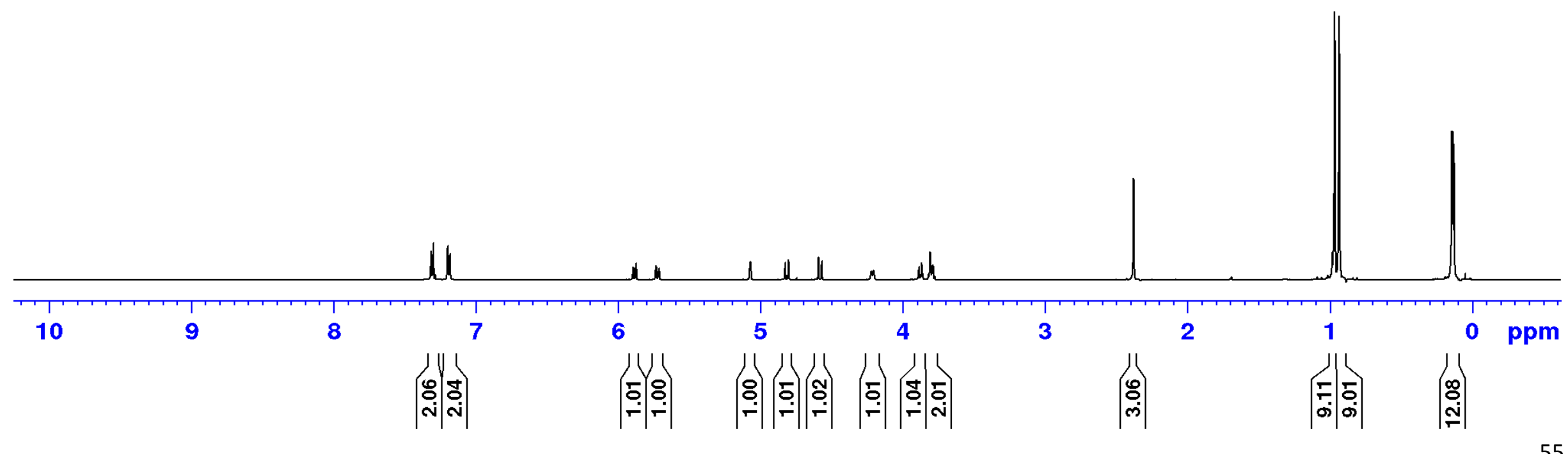




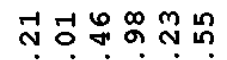

mิ

곤

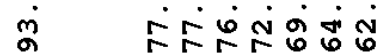

V111

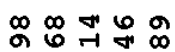

กู่

VI

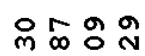

$>1 / 1$

1

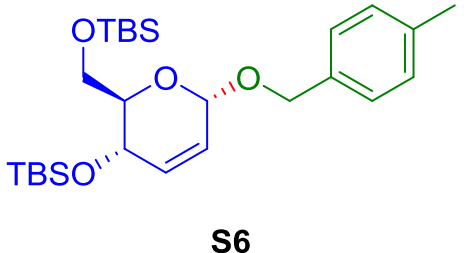

s6

${ }^{13} \mathrm{C}$ NMR (125 MHz, $\mathrm{CDCl}_{3}$ )

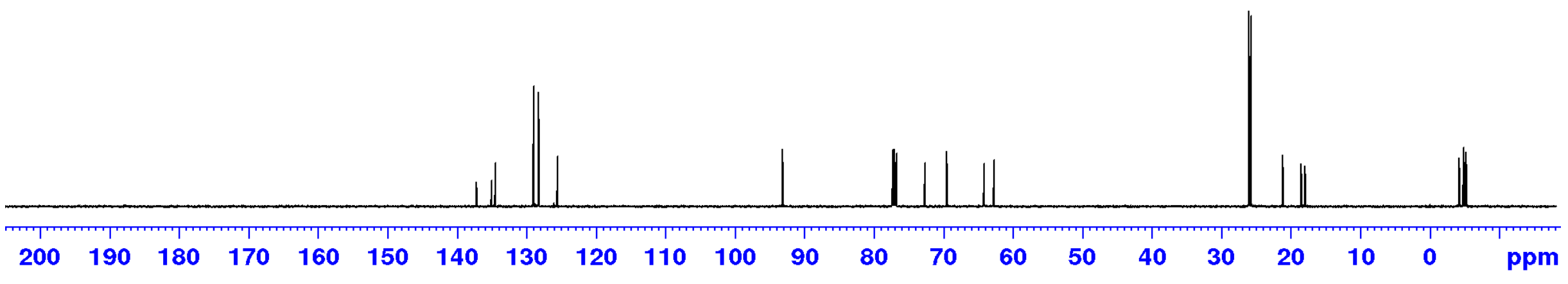




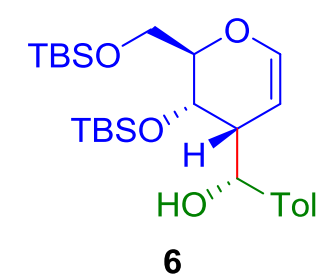

${ }^{1} \mathrm{H}$ NMR (400 MHz, $\mathrm{CDCl}_{3}$ )

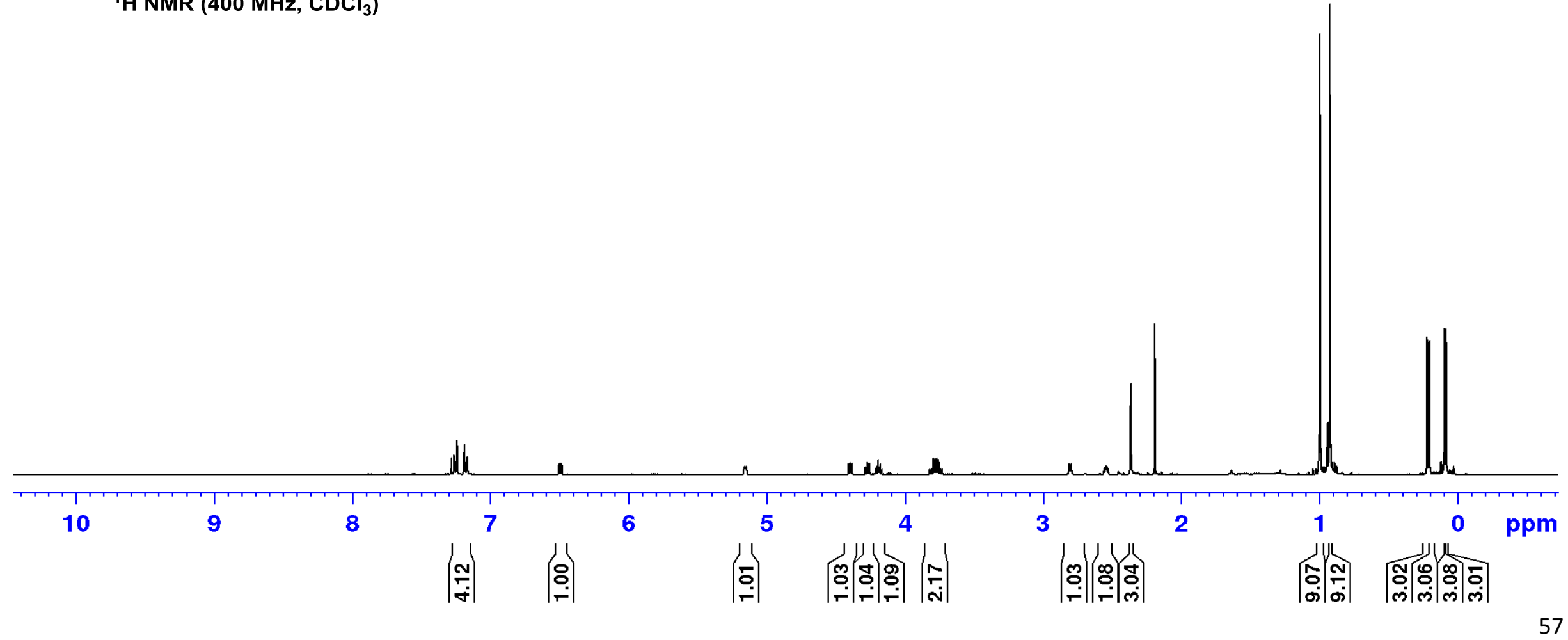




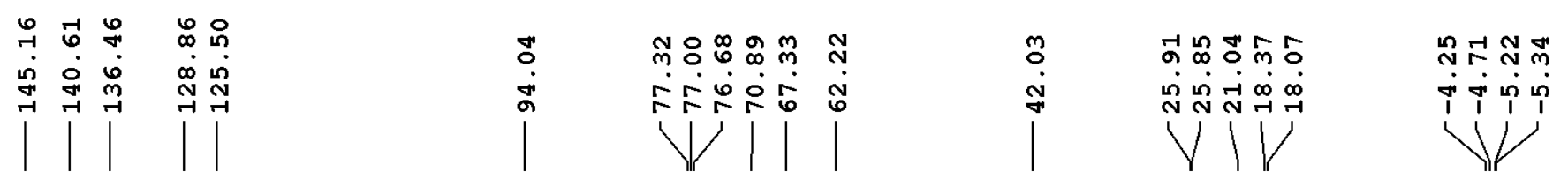

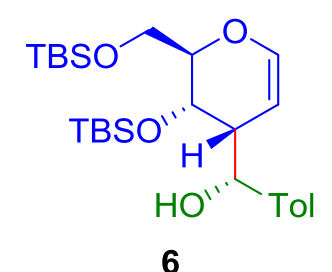

${ }^{13} \mathrm{C}$ NMR (100 MHz, $\left.\mathrm{CDCl}_{3}\right)$

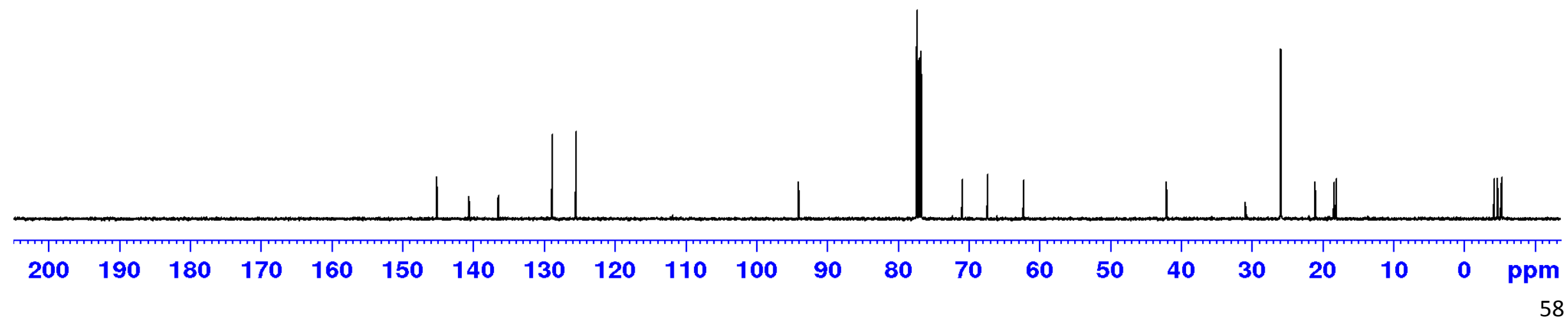



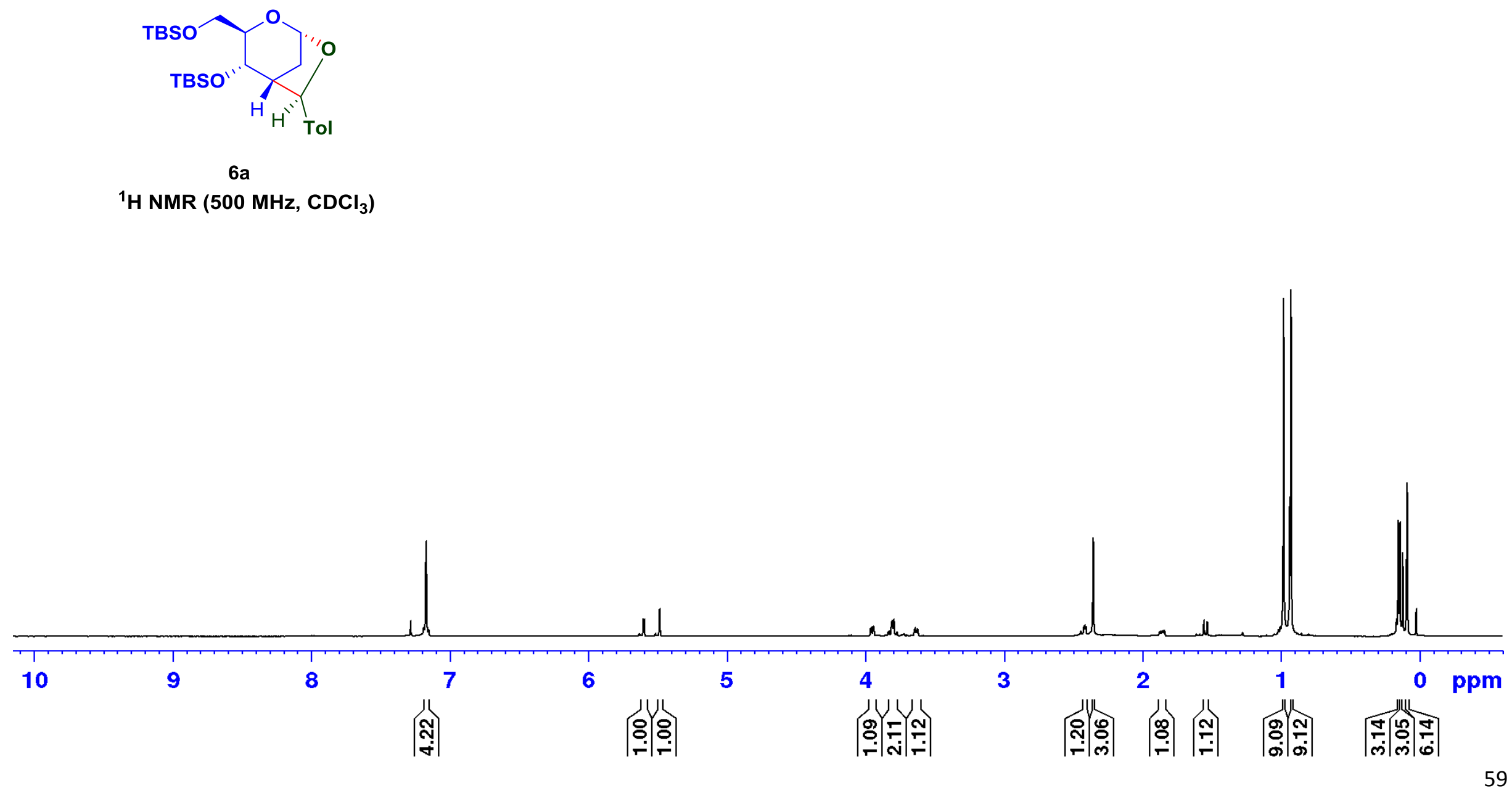


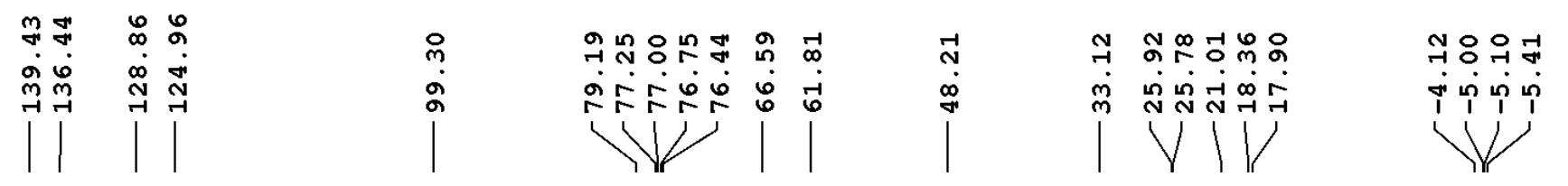

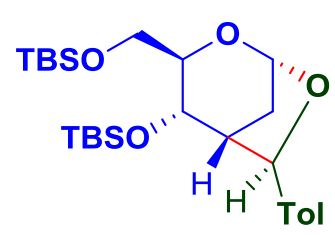

$6 a$

${ }^{13} \mathrm{C}$ NMR (125 MHz, $\mathrm{CDCl}_{3}$ )

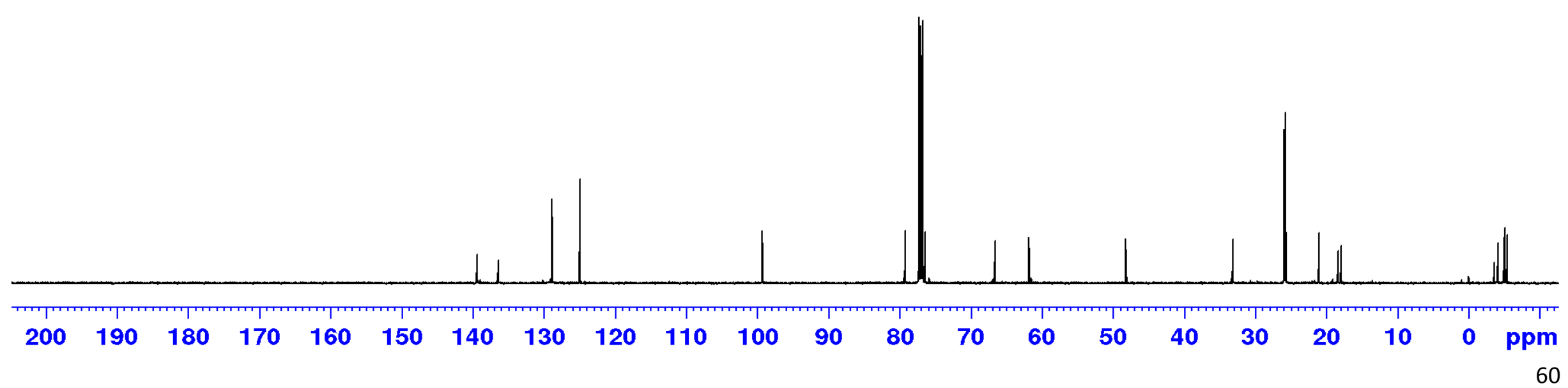




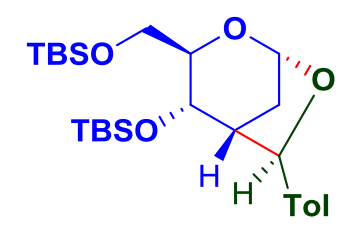

$6 a$

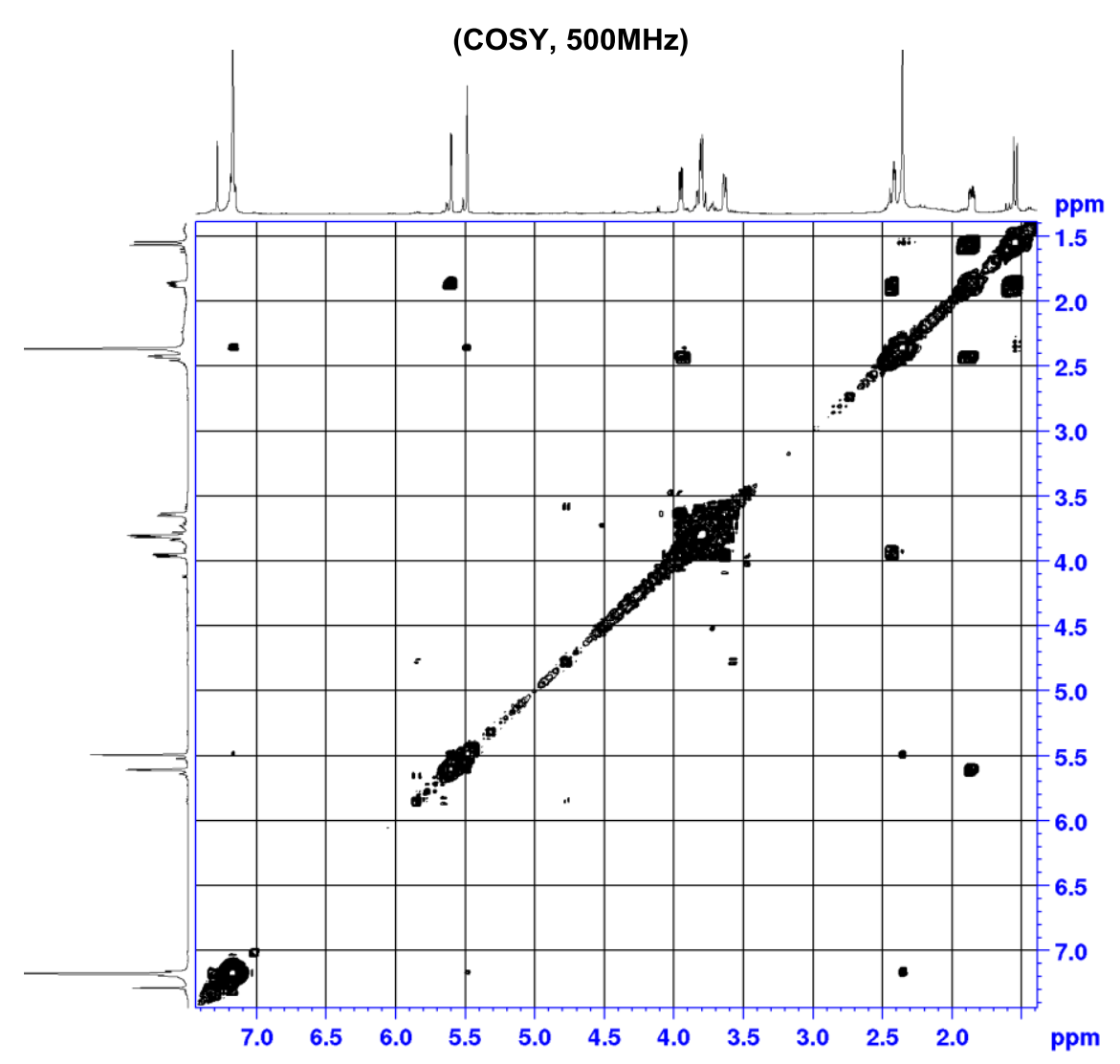

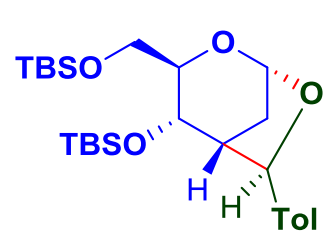

$6 a$

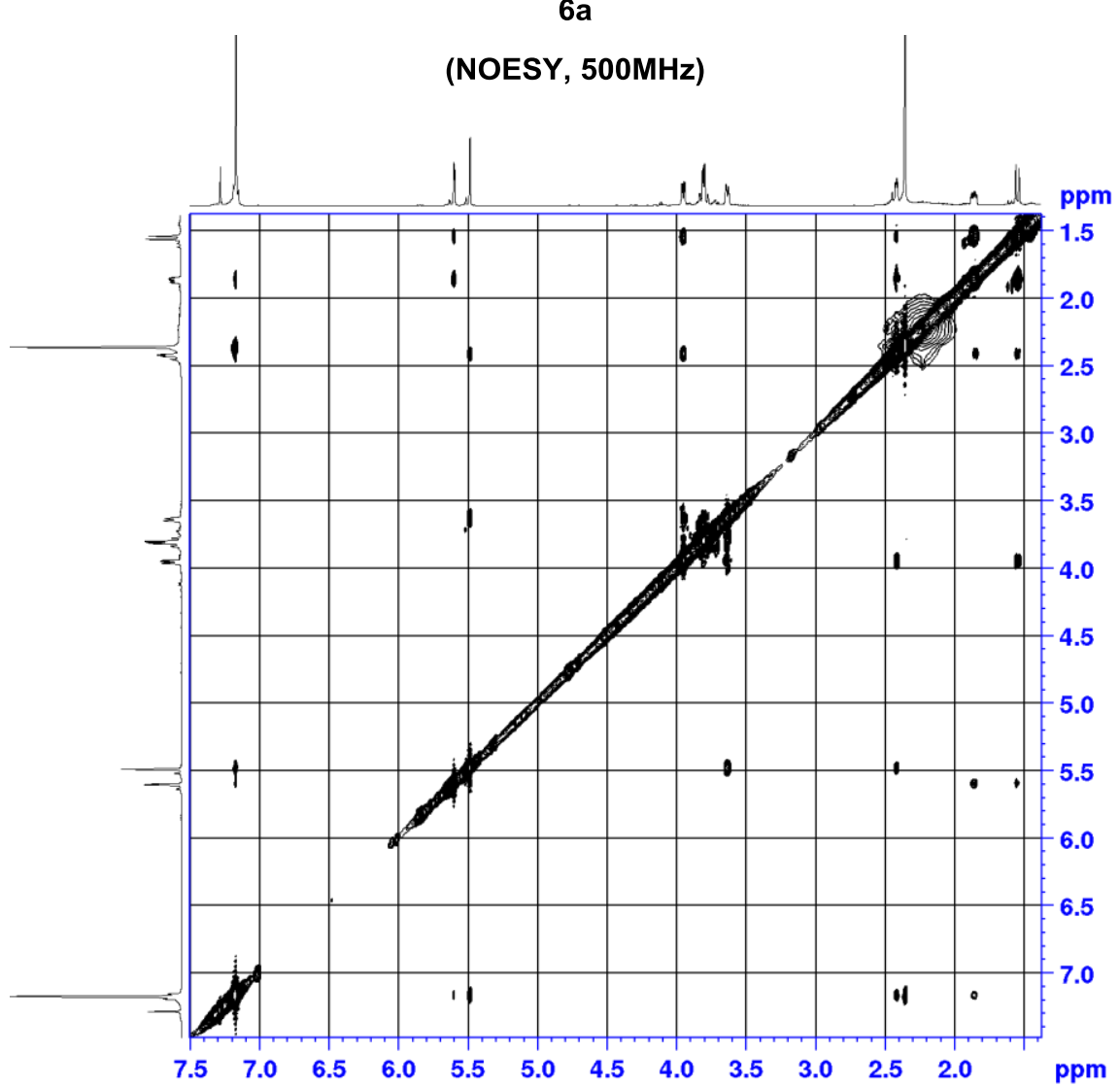




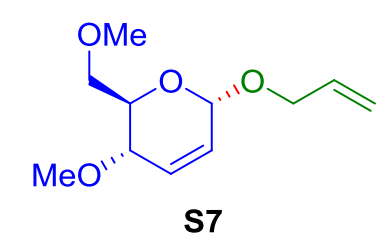

${ }^{1} \mathrm{H}$ NMR (500 $\mathrm{MHz} \mathrm{CDCl}_{3}$ )

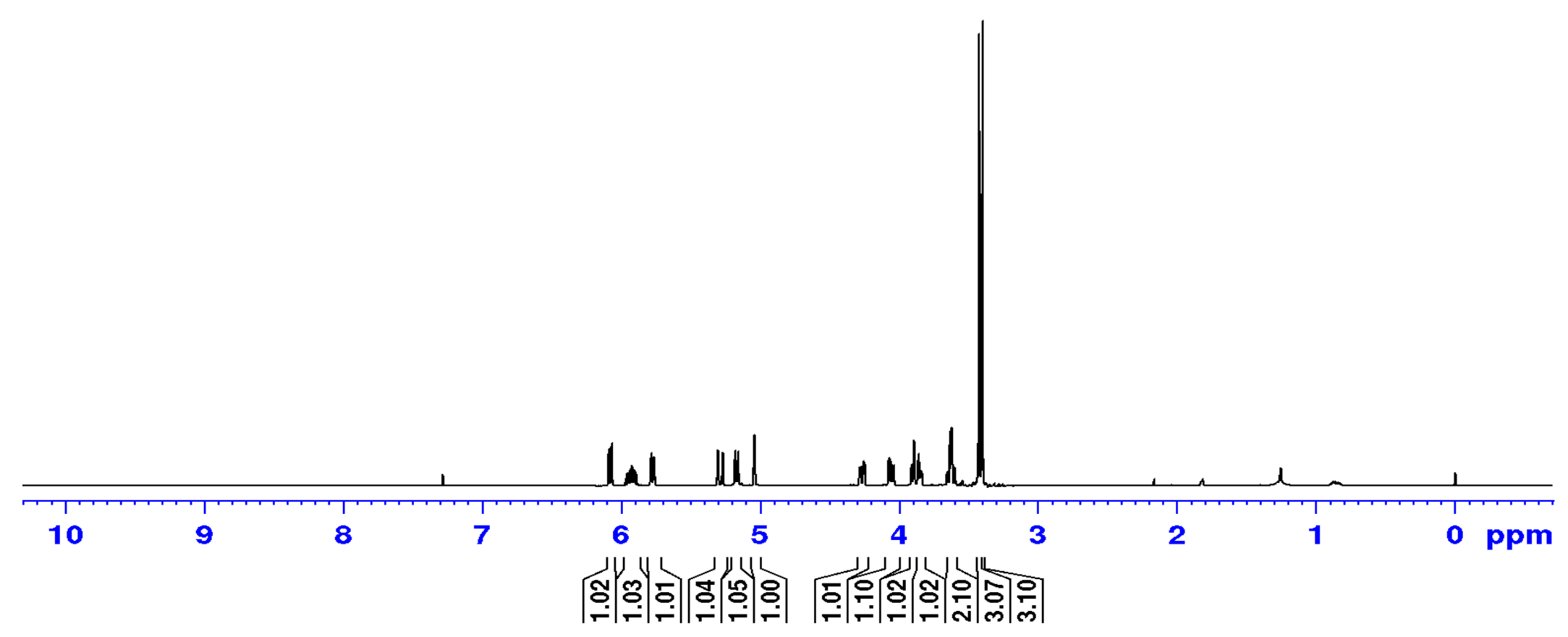



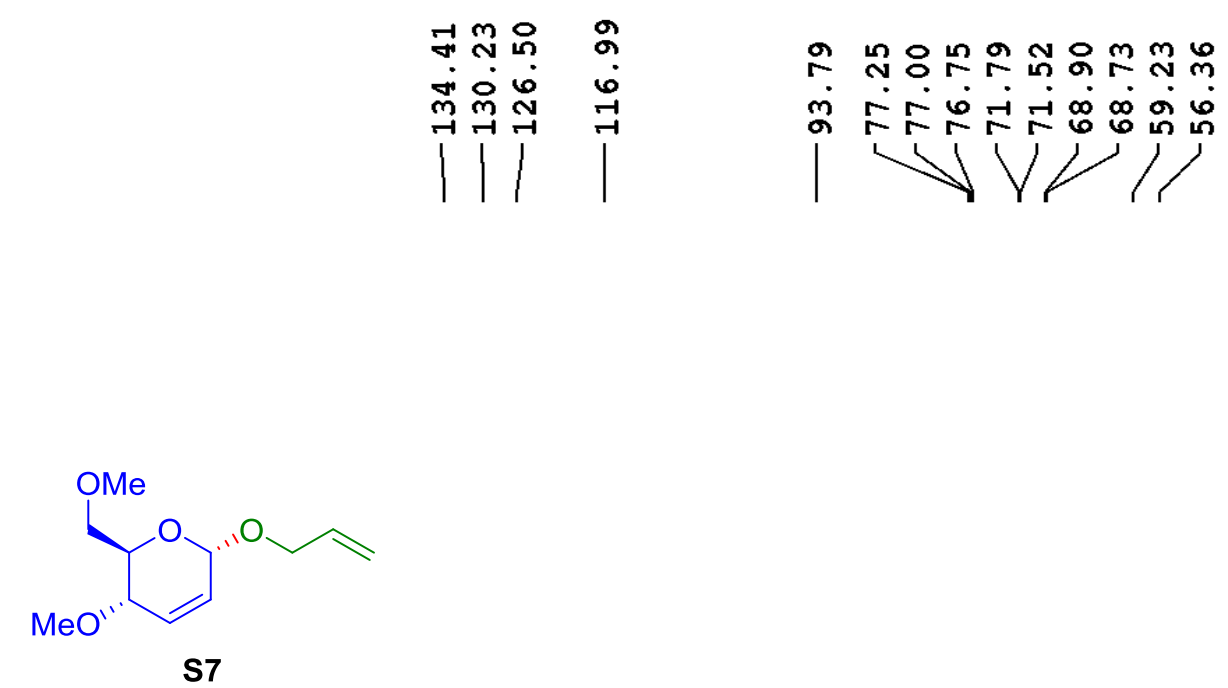

${ }^{13} \mathrm{C}$ NMR (125 MHz, $\mathrm{CDCl}_{3}$ )

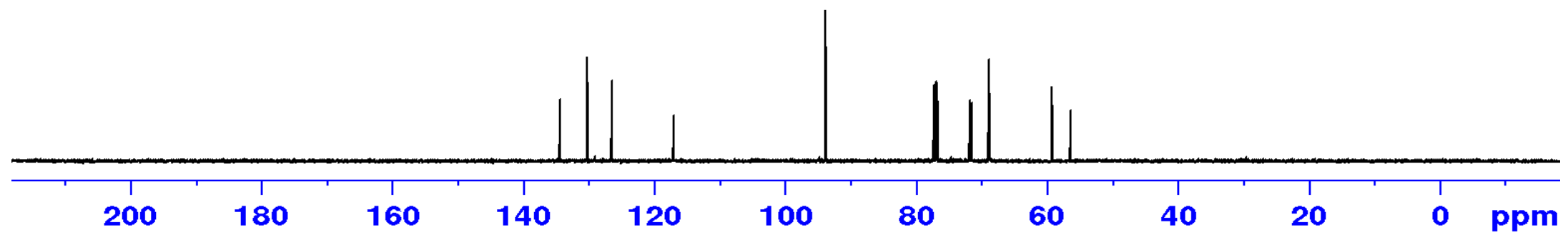




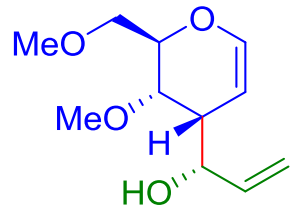

7

${ }^{1} \mathrm{H}$ NMR (500 MHz, $\mathrm{CDCl}_{3}$ )

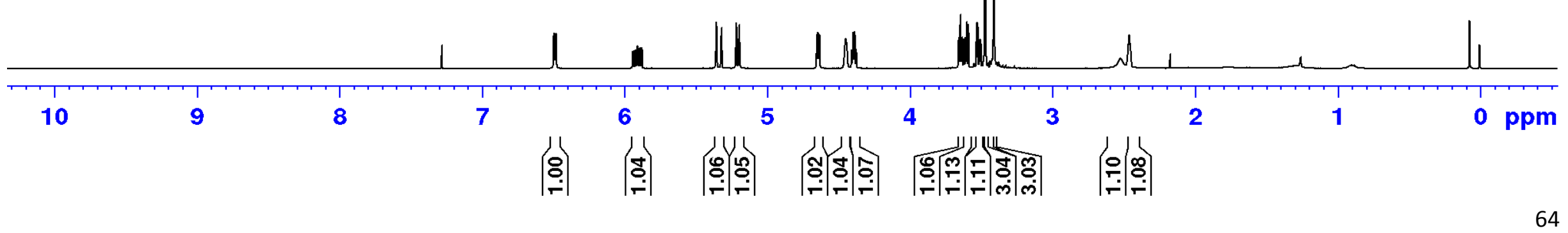




| | la

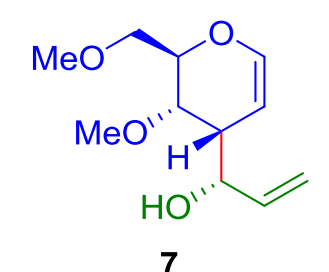

${ }^{13} \mathrm{C} \mathrm{NMR}\left(125 \mathrm{MHz}, \mathrm{CDCl}_{3}\right)$

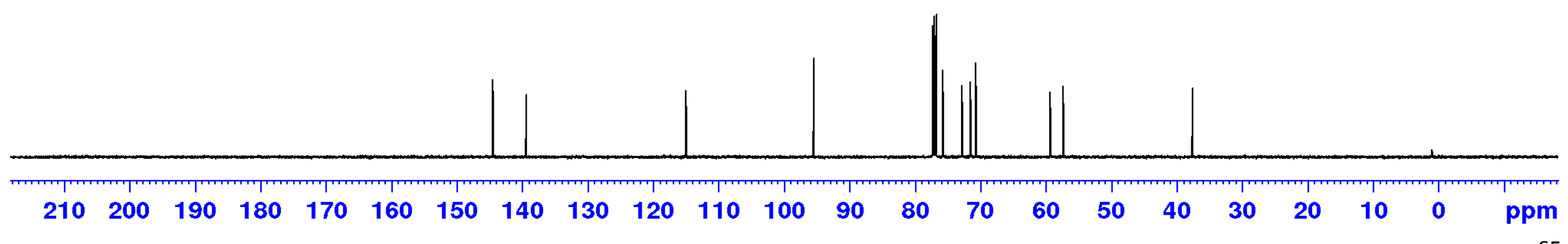




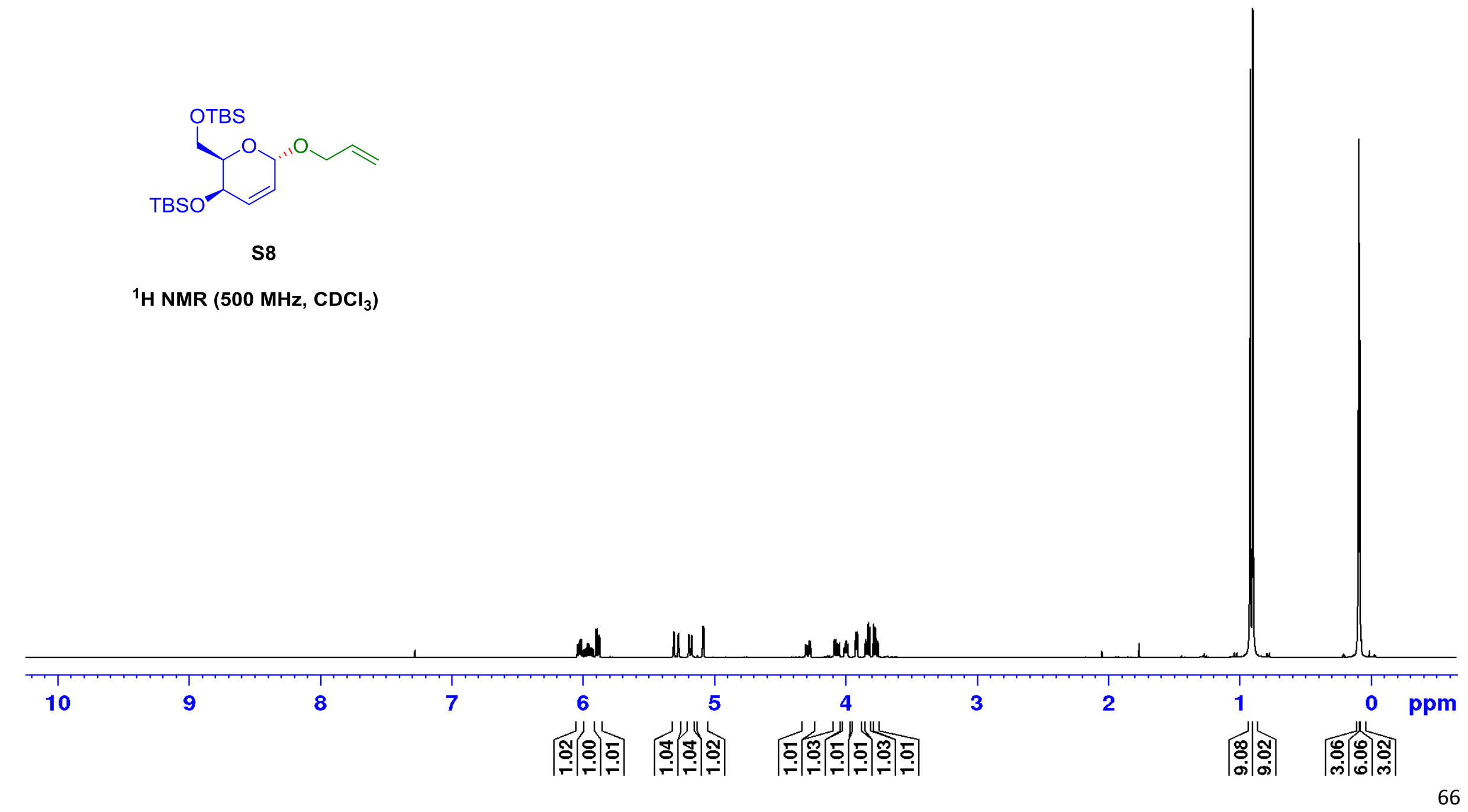




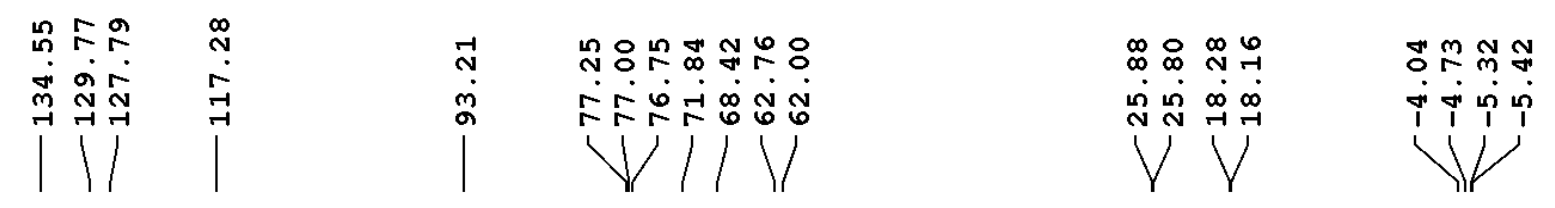

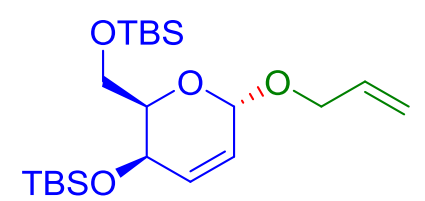

S8

${ }^{13} \mathrm{C}$ NMR (125 MHz, $\mathrm{CDCl}_{3}$ )

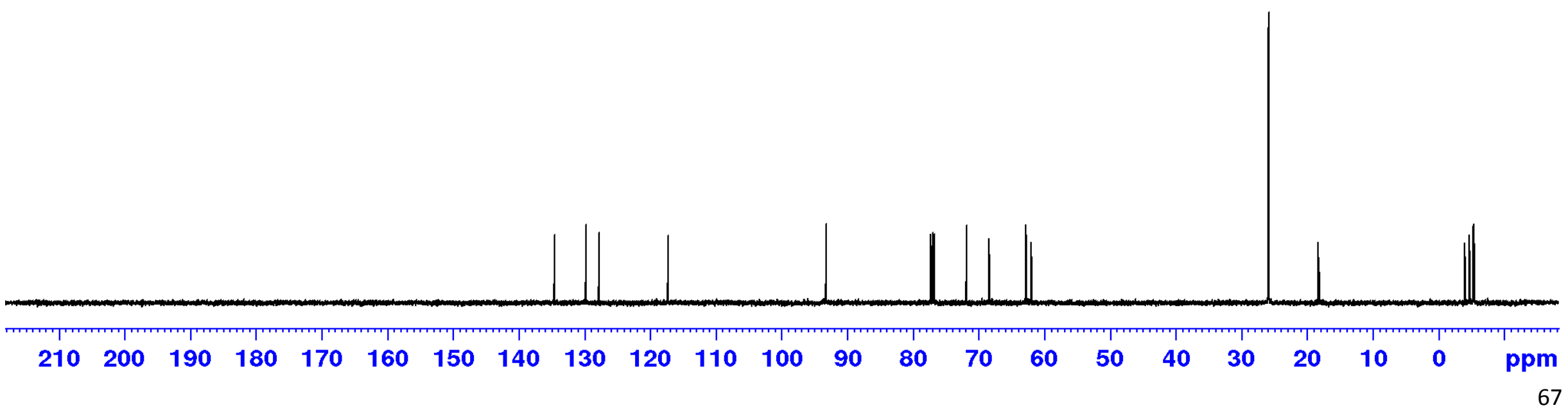




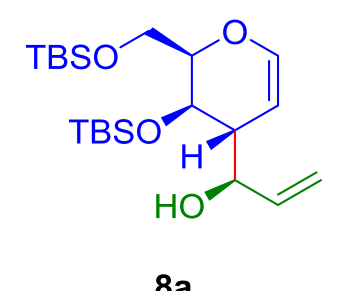

${ }^{1} \mathrm{H}$ NMR (500 MHz, $\mathrm{CDCl}_{3}$ )

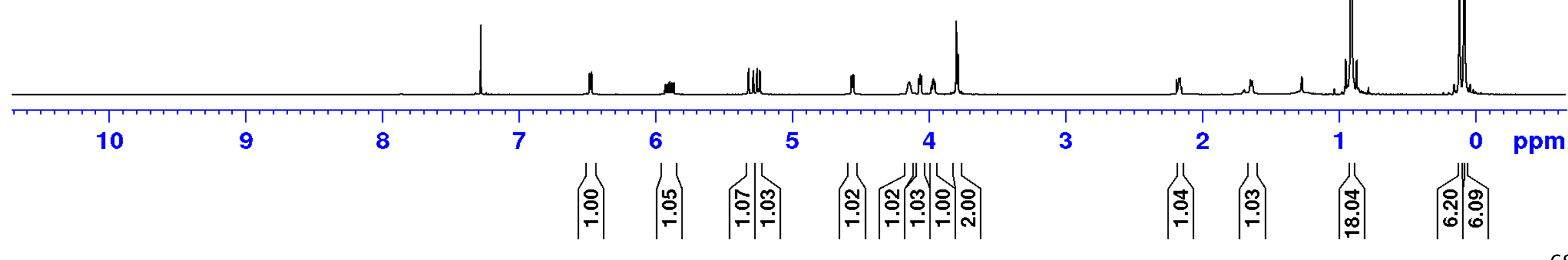




|

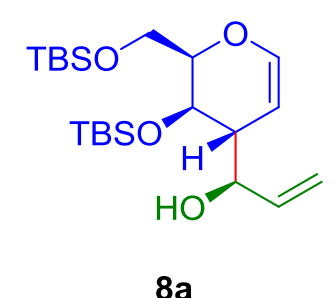

${ }^{13} \mathrm{C}$ NMR (125 MHz, $\mathrm{CDCl}_{3}$ )

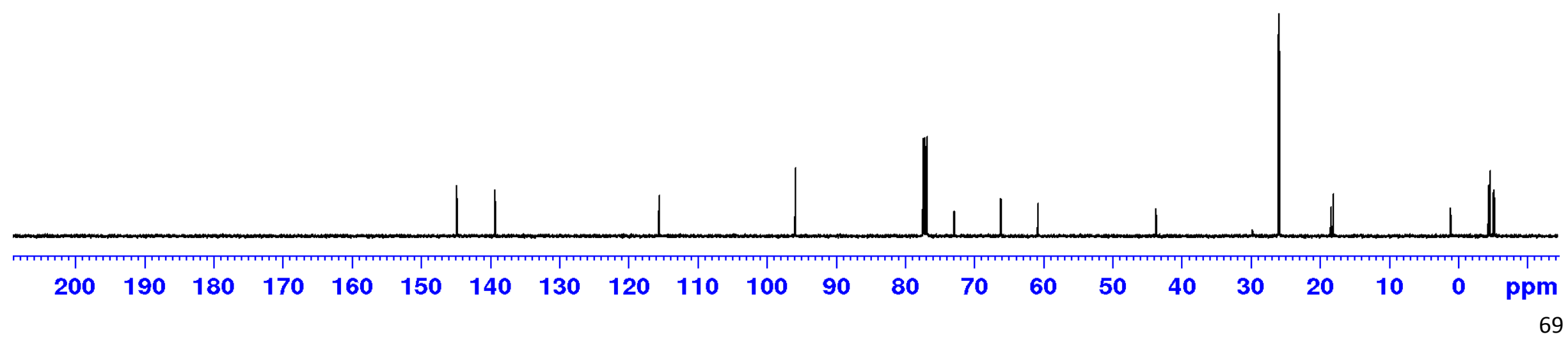




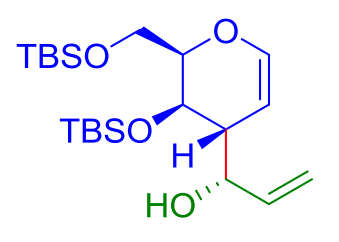

$8 b$

${ }^{1} \mathrm{H}$ NMR (500 MHz, $\mathrm{CDCl}_{3}$ )

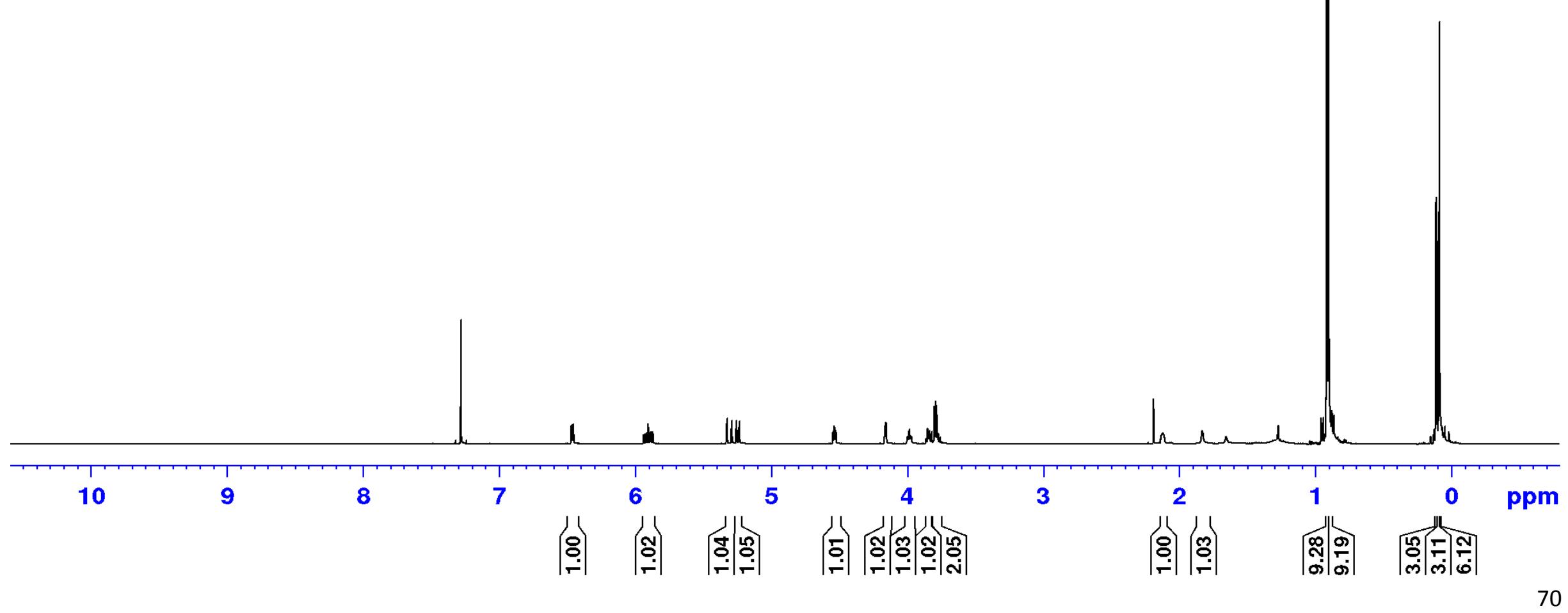




年

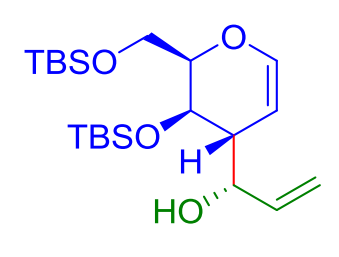

$8 b$

${ }^{13} \mathrm{C}$ NMR (125 MHz, $\mathrm{CDCl}_{3}$ )

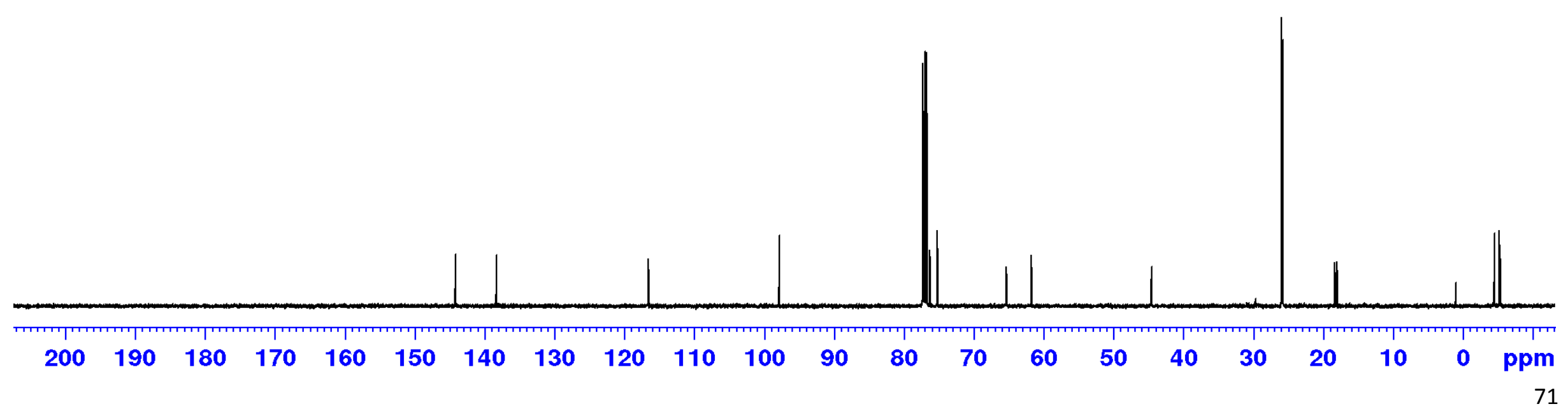




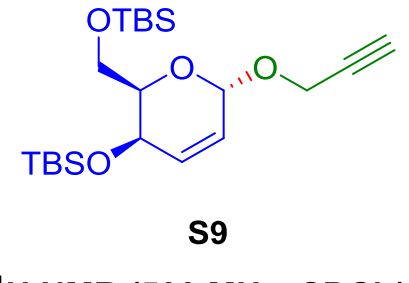

${ }^{1} \mathrm{H}$ NMR (500 MHz, $\mathrm{CDCl}_{3}$ )

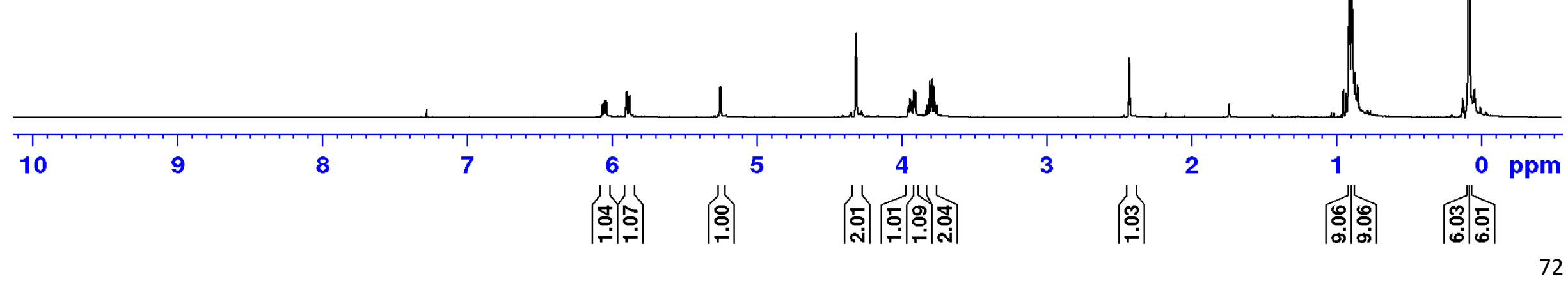




|l

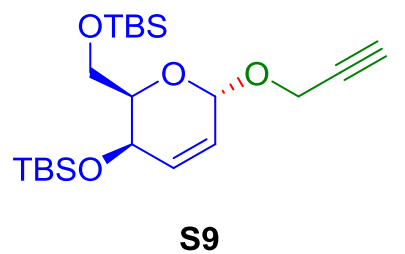

${ }^{13} \mathrm{C}$ NMR (125 MHz, $\mathrm{CDCl}_{3}$ )

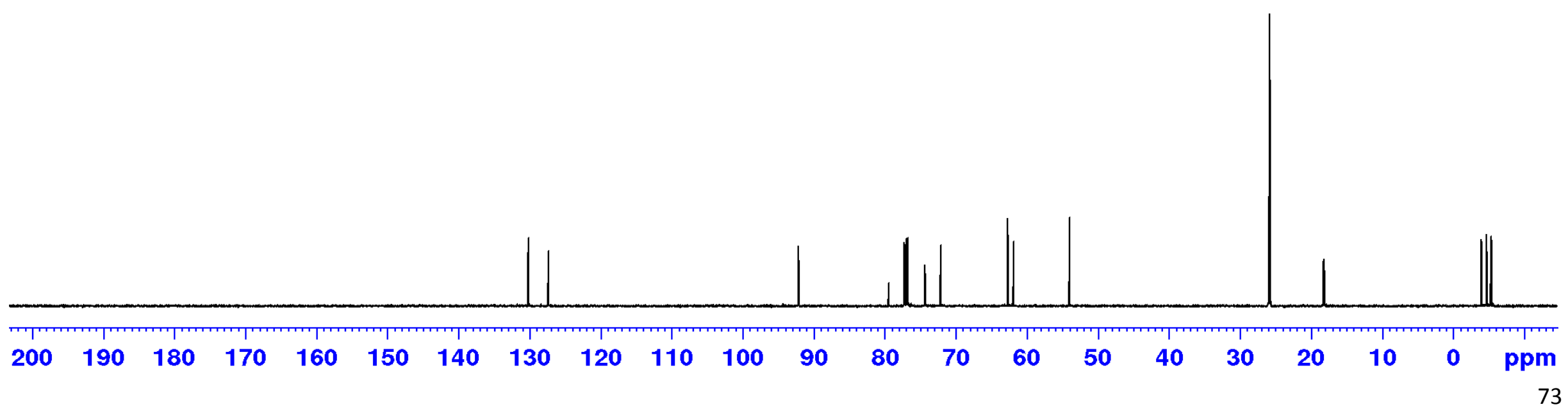




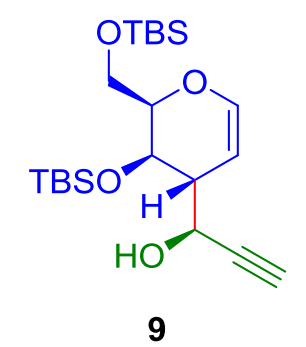

${ }^{1} \mathrm{H}$ NMR (400 MHz, $\left.\mathrm{CDCl}_{3}\right)$

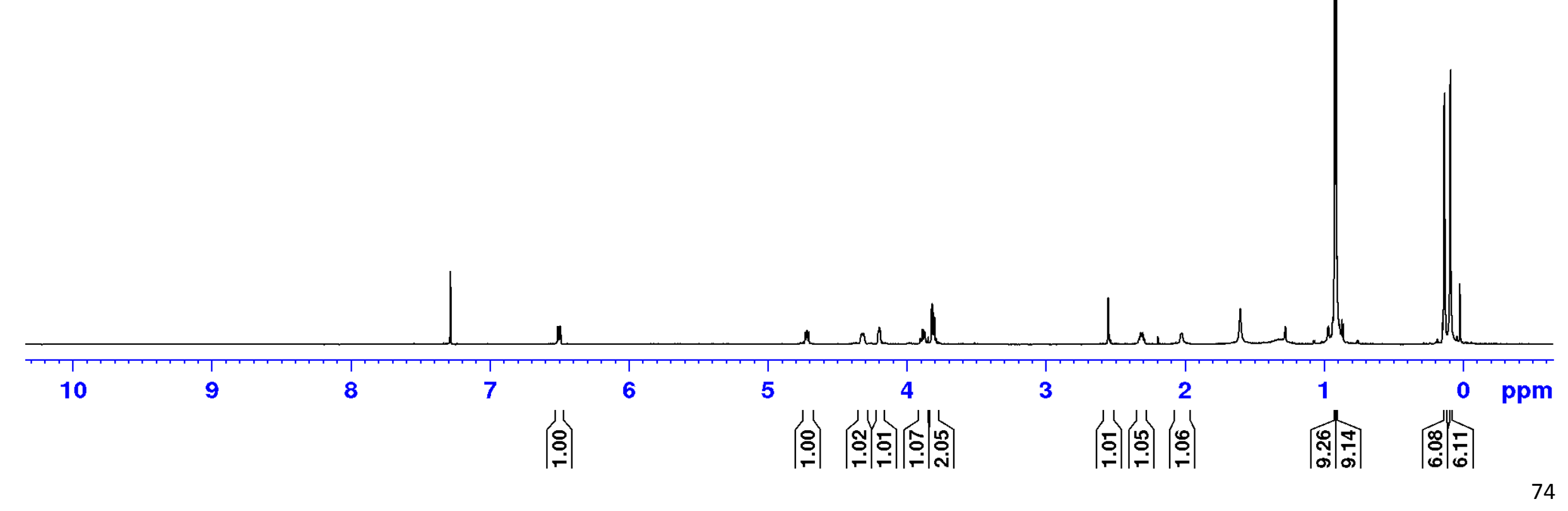



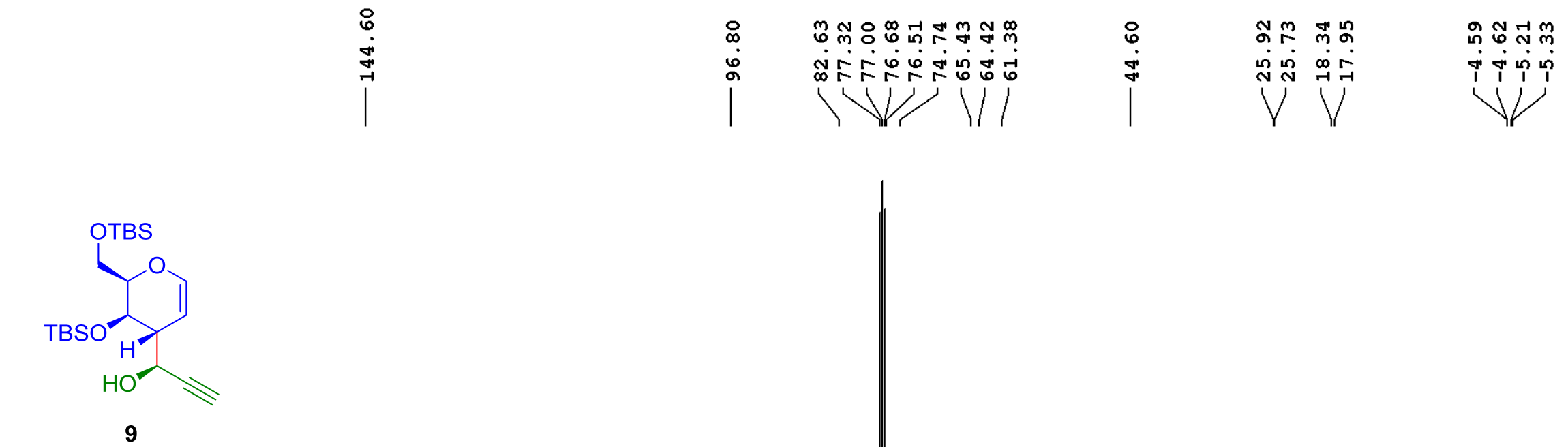

${ }^{13} \mathrm{C}$ NMR (100 MHz, $\mathrm{CDCl}_{3}$ )

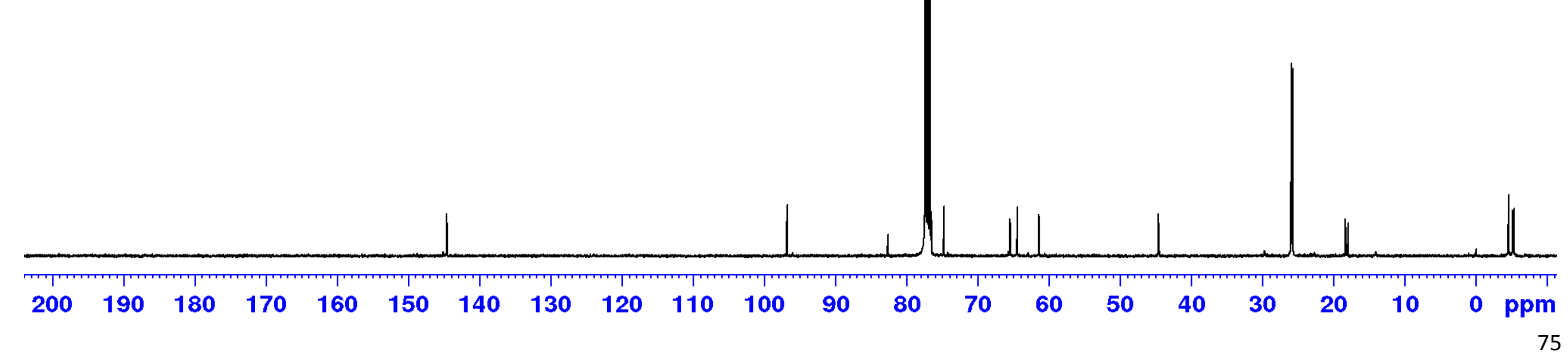




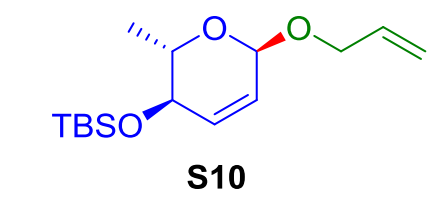

${ }^{1} \mathrm{H}$ NMR (500 MHz, $\mathrm{CDCl}_{3}$ )

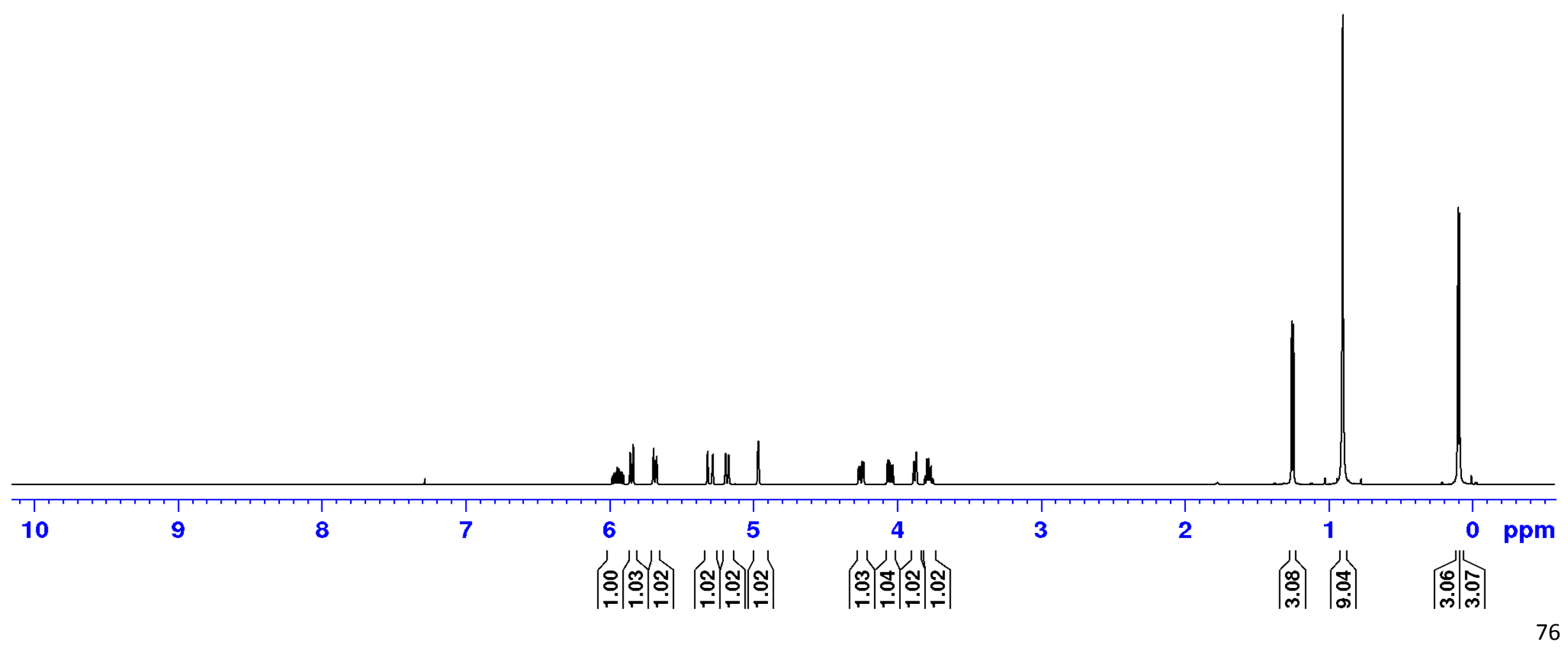



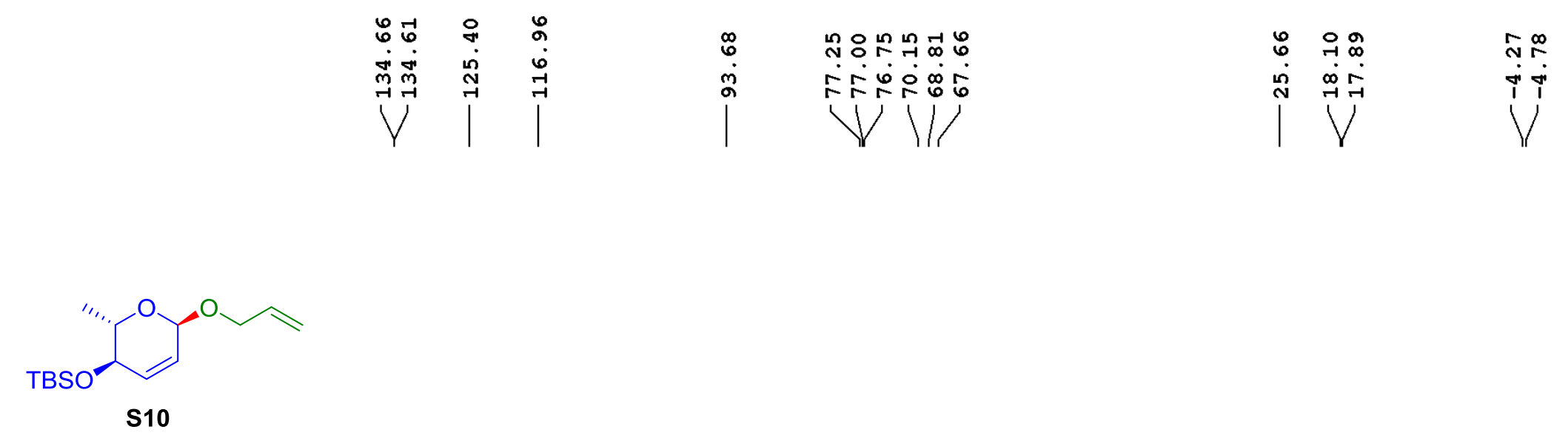

${ }^{13} \mathrm{C}$ NMR (125 MHz, $\mathrm{CDCl}_{3}$ )

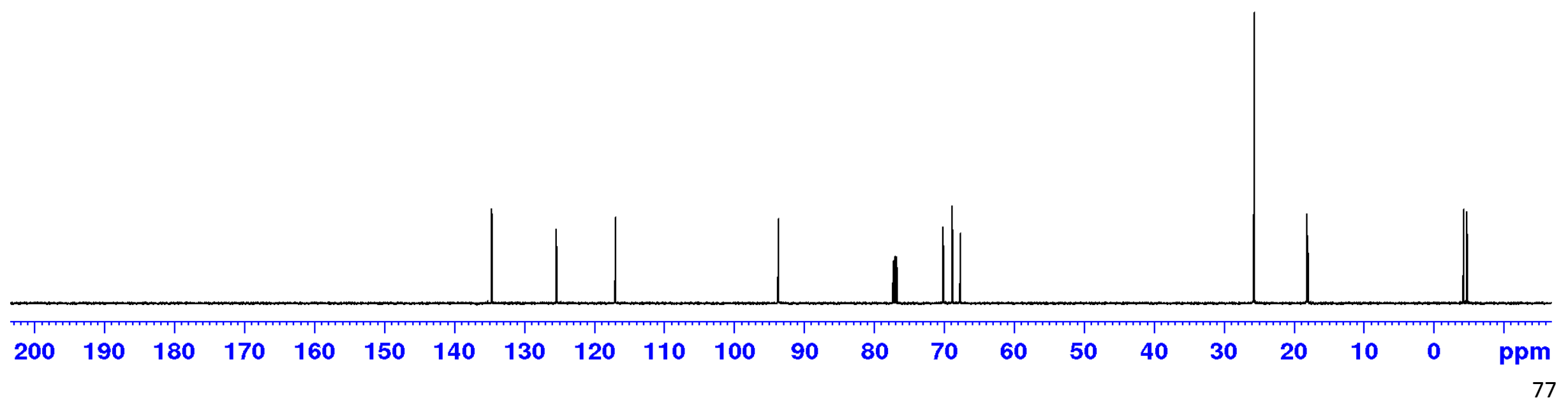




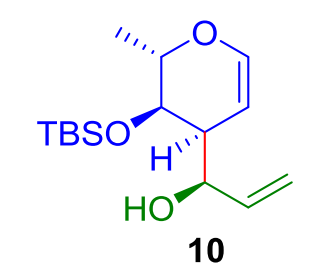

${ }^{1} \mathrm{H}$ NMR (400 MHz, $\mathrm{CDCl}_{3}$ )

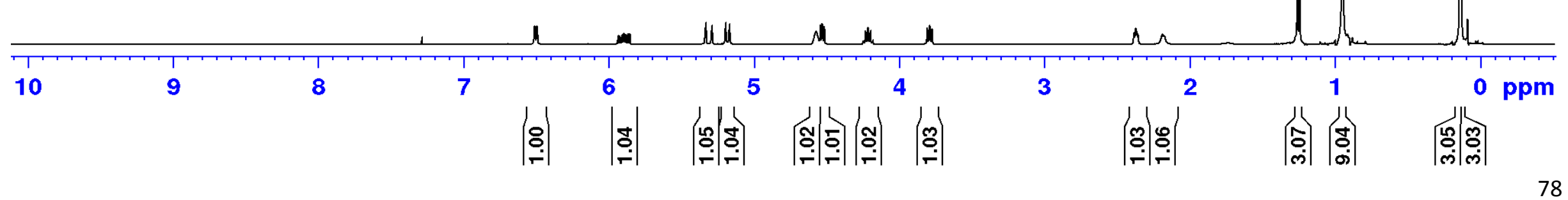




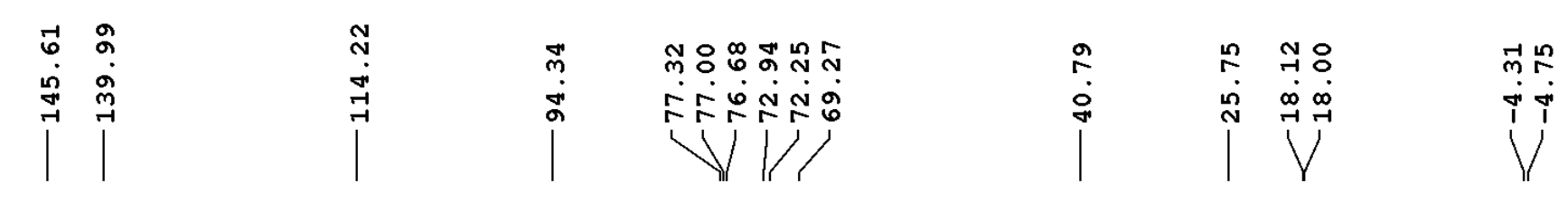

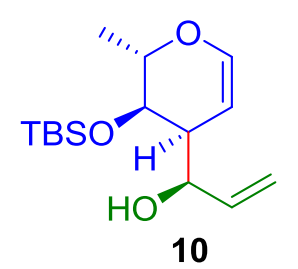

${ }^{13} \mathrm{C}$ NMR (100 $\left.\mathrm{MHz}, \mathrm{CDCl}_{3}\right)$

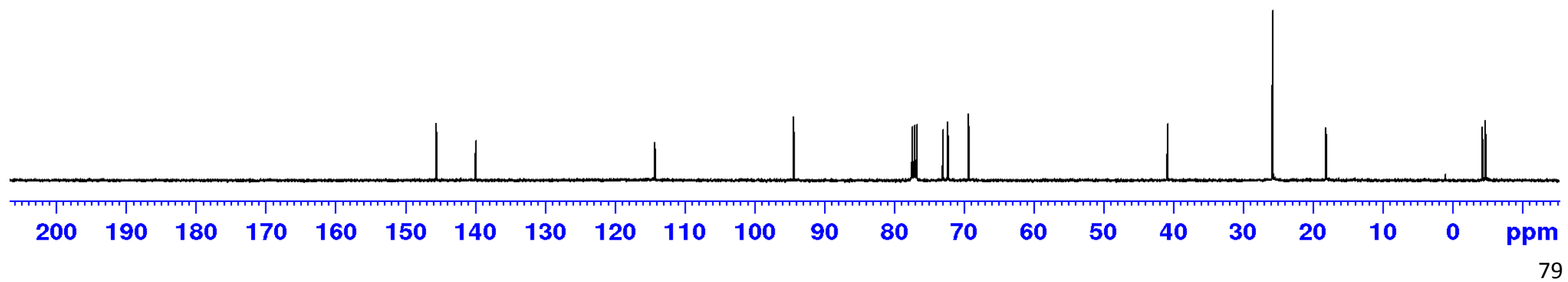




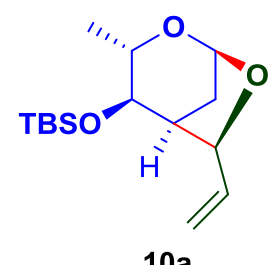

${ }^{1} \mathrm{H}$ NMR (500 MHz, $\mathrm{CDCl}_{3}$ )

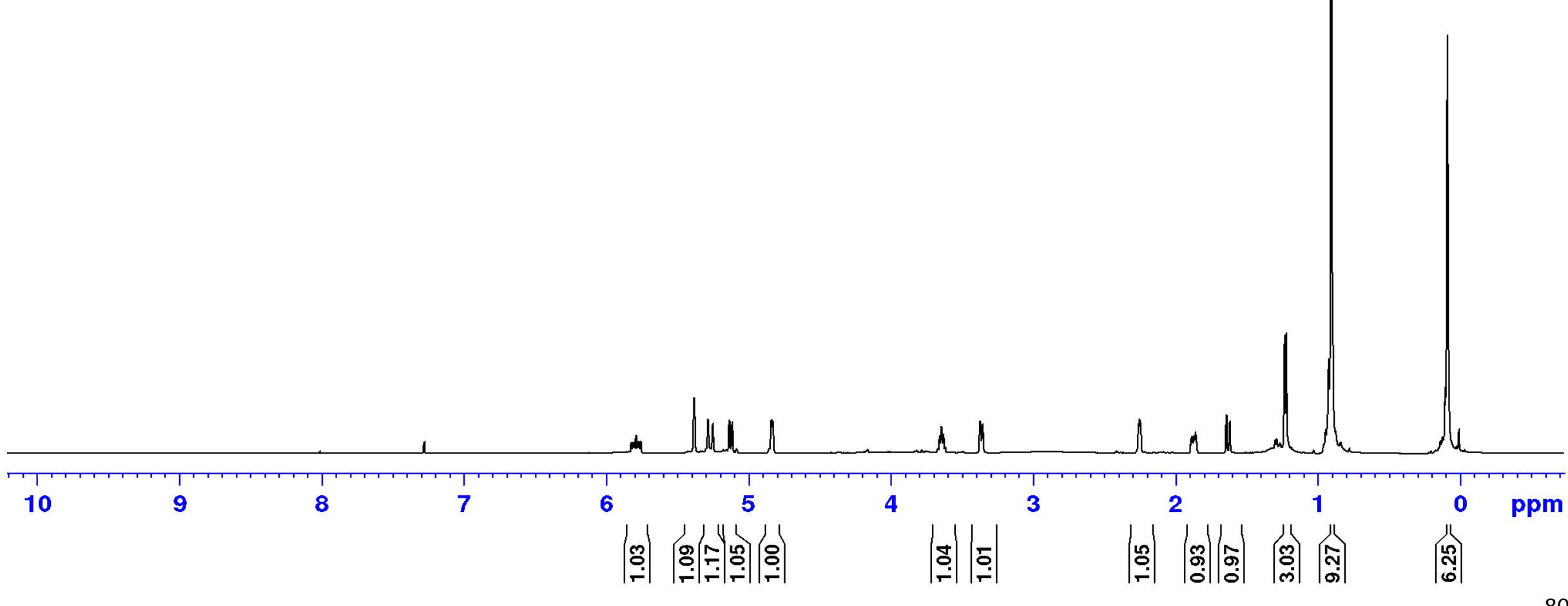




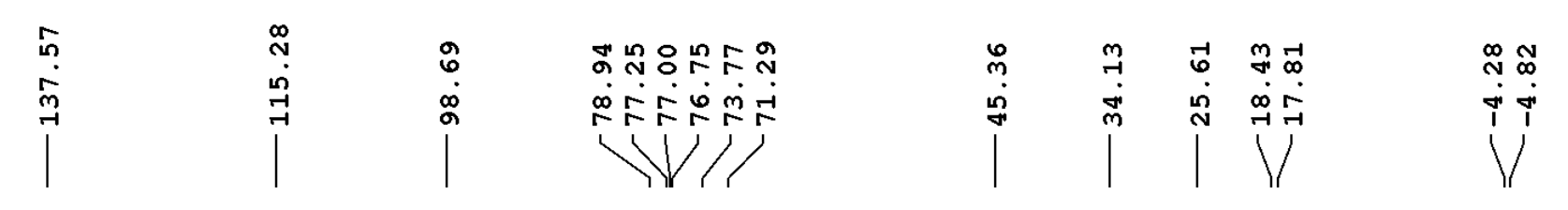

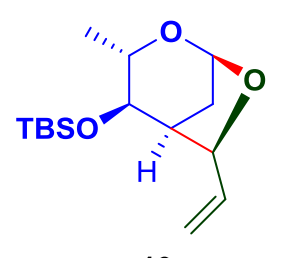

10a

${ }^{13} \mathrm{C}$ NMR (125 MHz, $\mathrm{CDCl}_{3}$ )

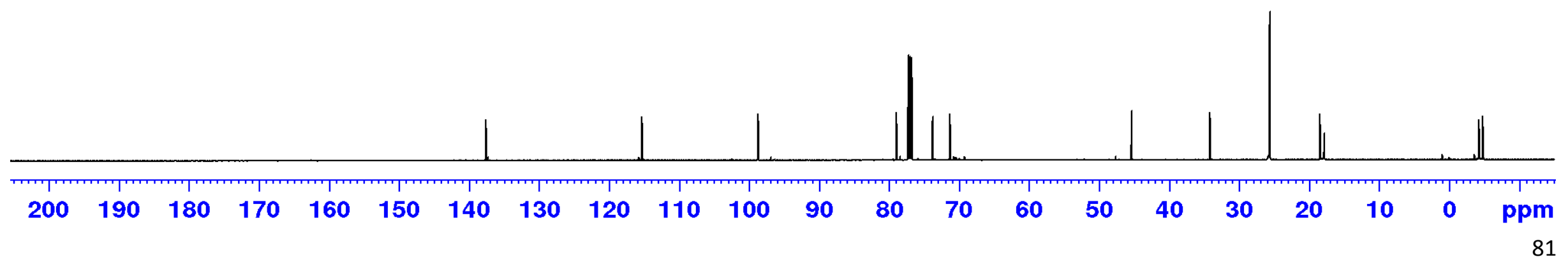




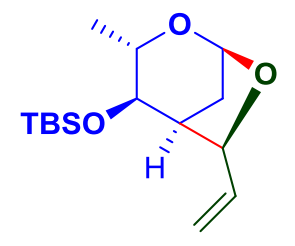

$10 a$

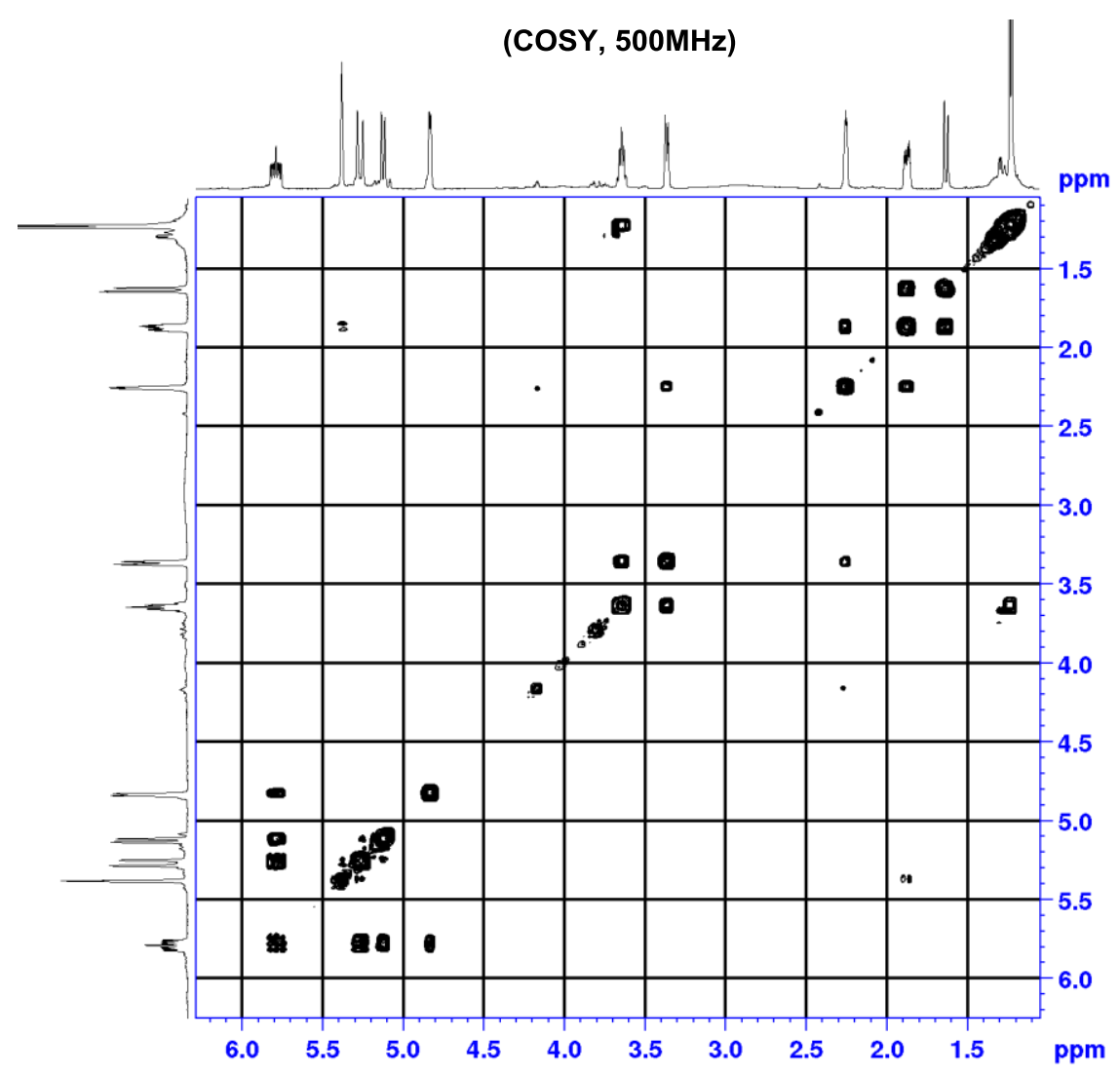

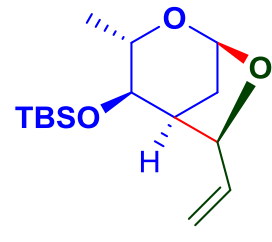

10a

(NOESY, 500MHz)

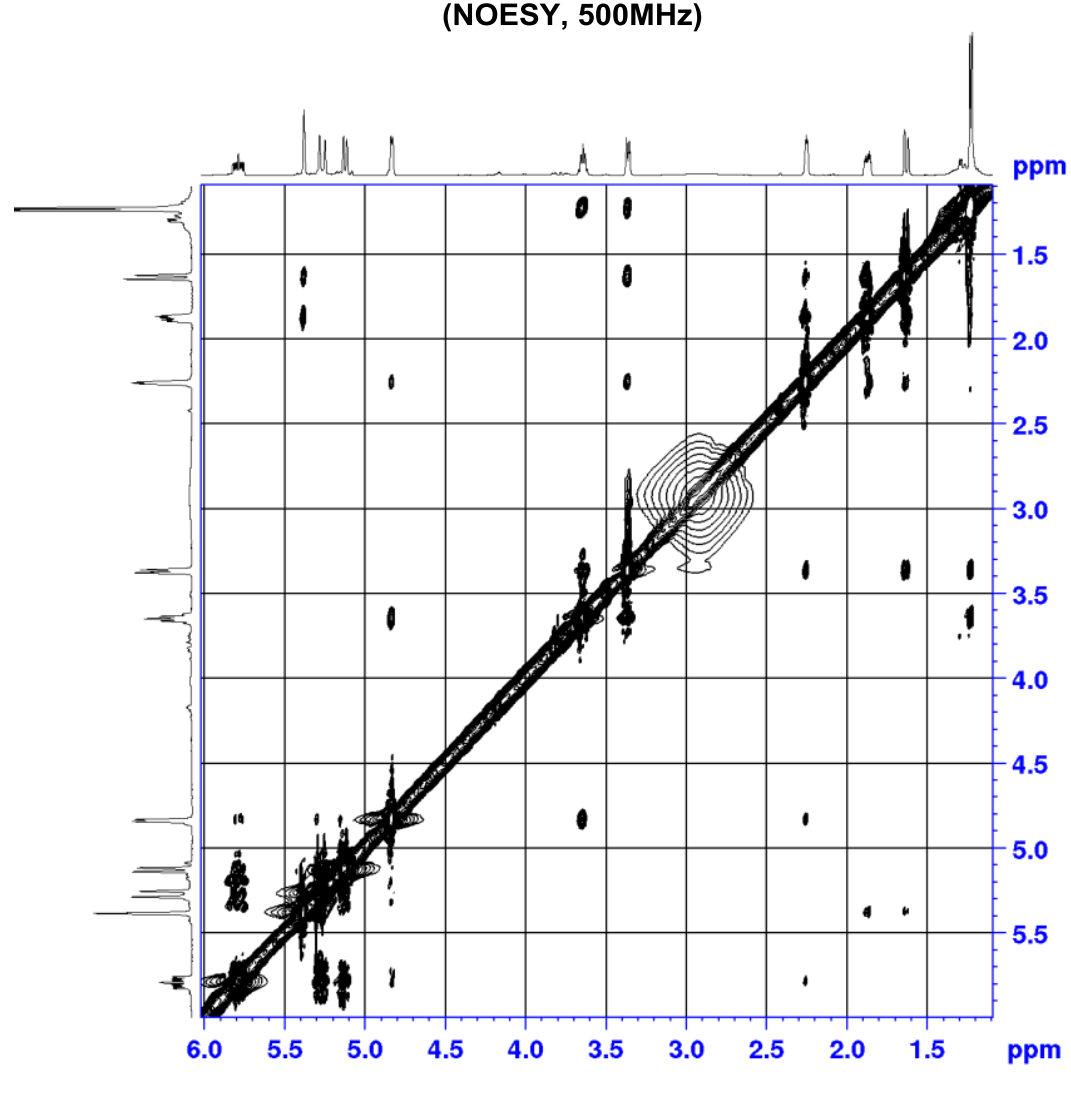




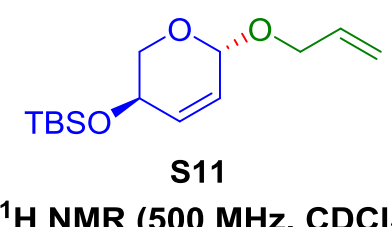

${ }^{1} \mathrm{H}$ NMR (500 MHz, $\mathrm{CDCl}_{3}$ )

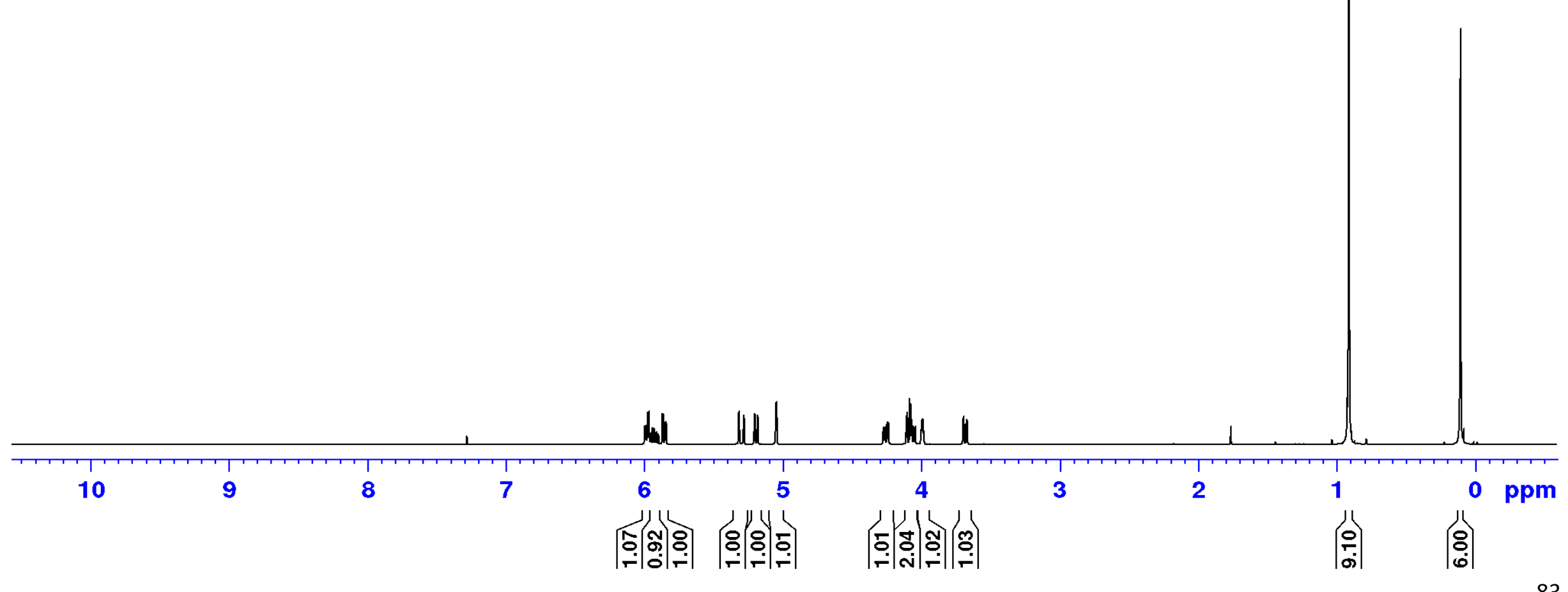




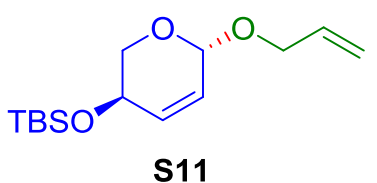

${ }^{13} \mathrm{C}$ NMR (125 MHz, $\mathrm{CDCl}_{3}$ )

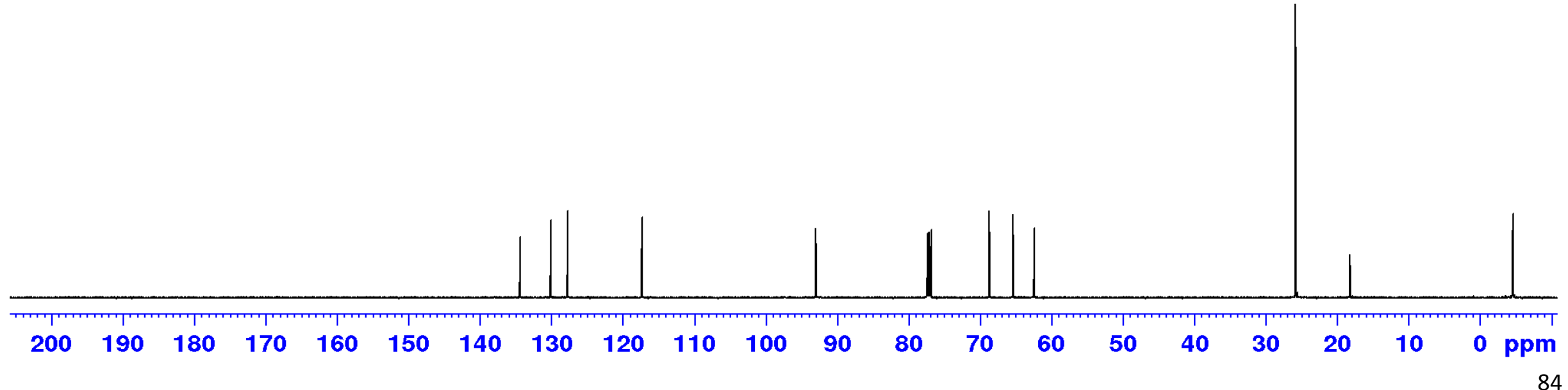




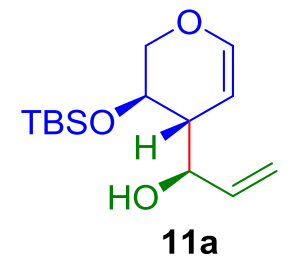

${ }^{1} \mathrm{H}$ NMR (500 MHz, $\mathrm{CDCl}_{3}$ )

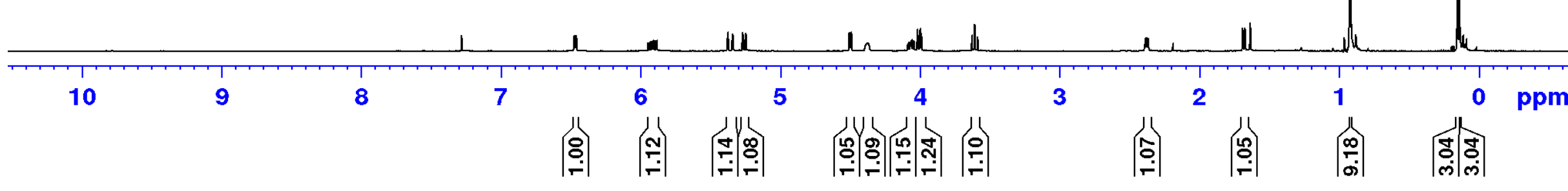




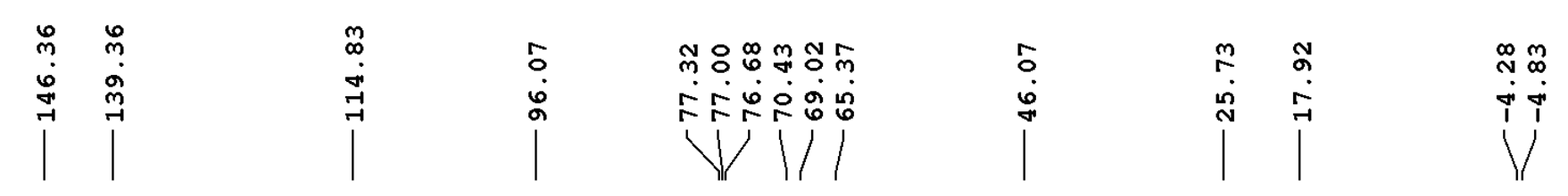

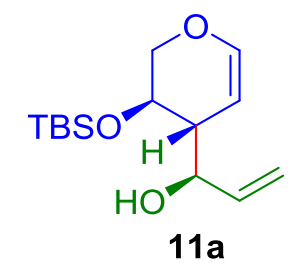

${ }^{13} \mathrm{C}$ NMR (100 MHz, $\mathrm{CDCl}_{3}$ )

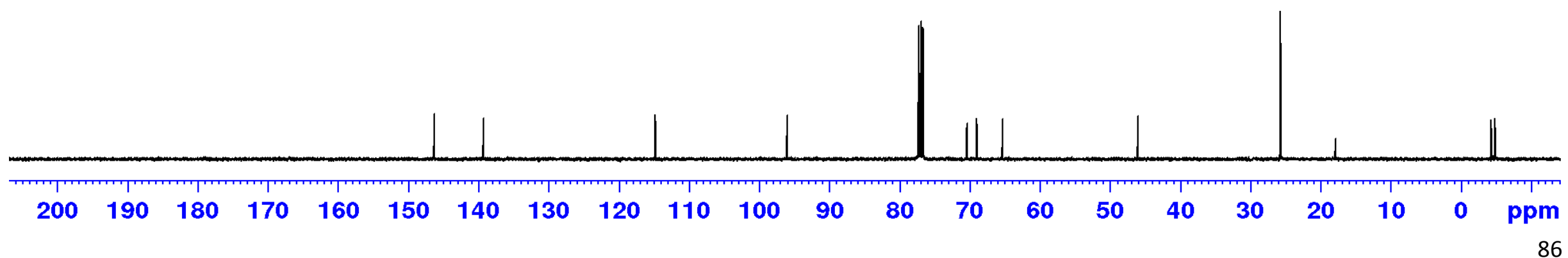




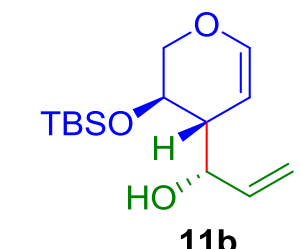

${ }^{1} \mathrm{H}$ NMR (500 MHz, $\mathrm{CDCl}_{3}$ )

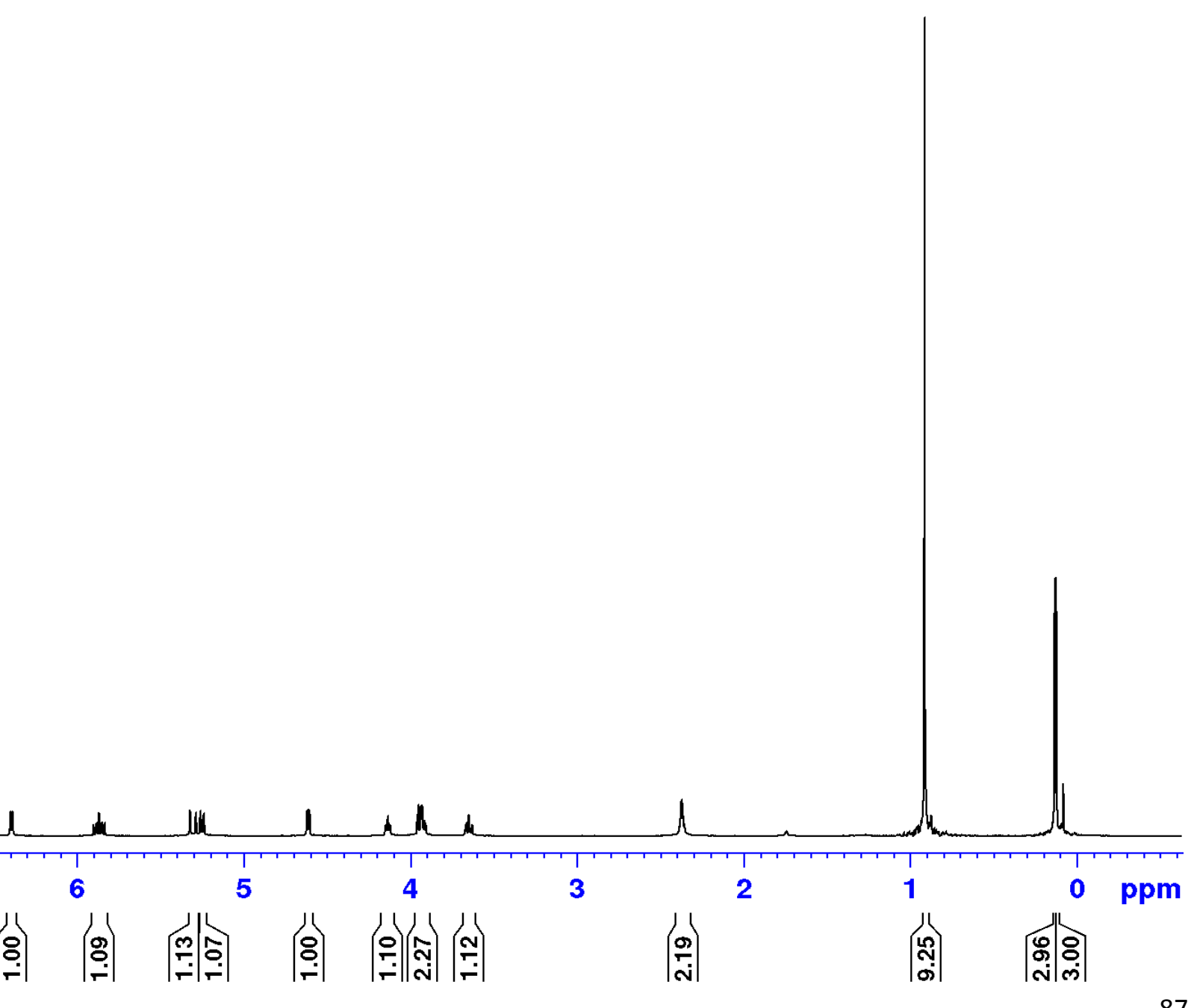




|l|

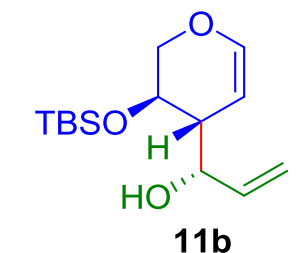

${ }^{13} \mathrm{C}$ NMR (125 MHz, $\mathrm{CDCl}_{3}$ )

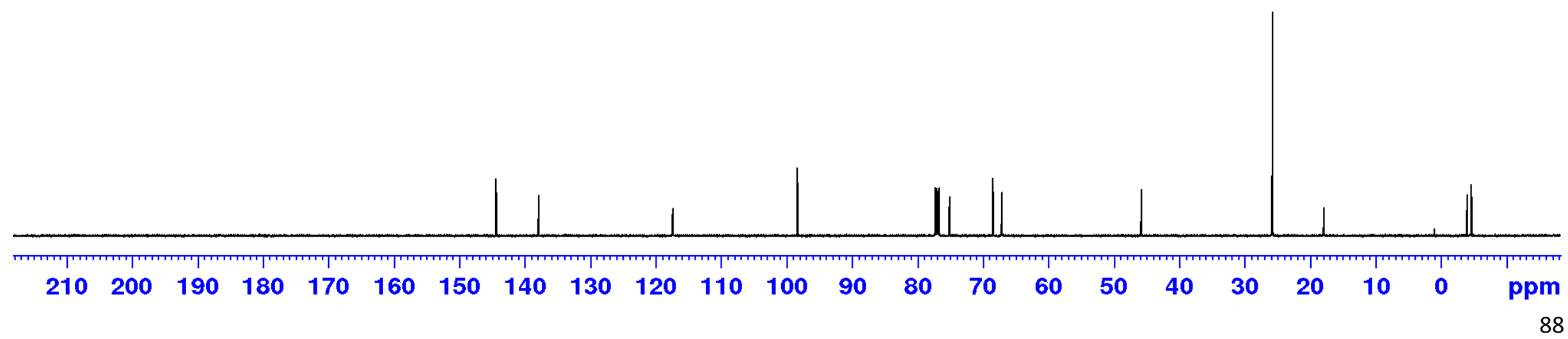




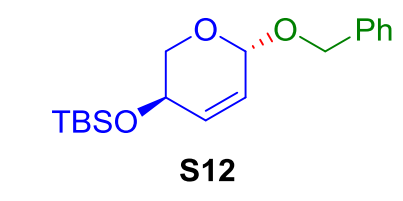

${ }^{1} \mathrm{H}$ NMR (400 MHz, $\mathrm{CDCl}_{3}$ )

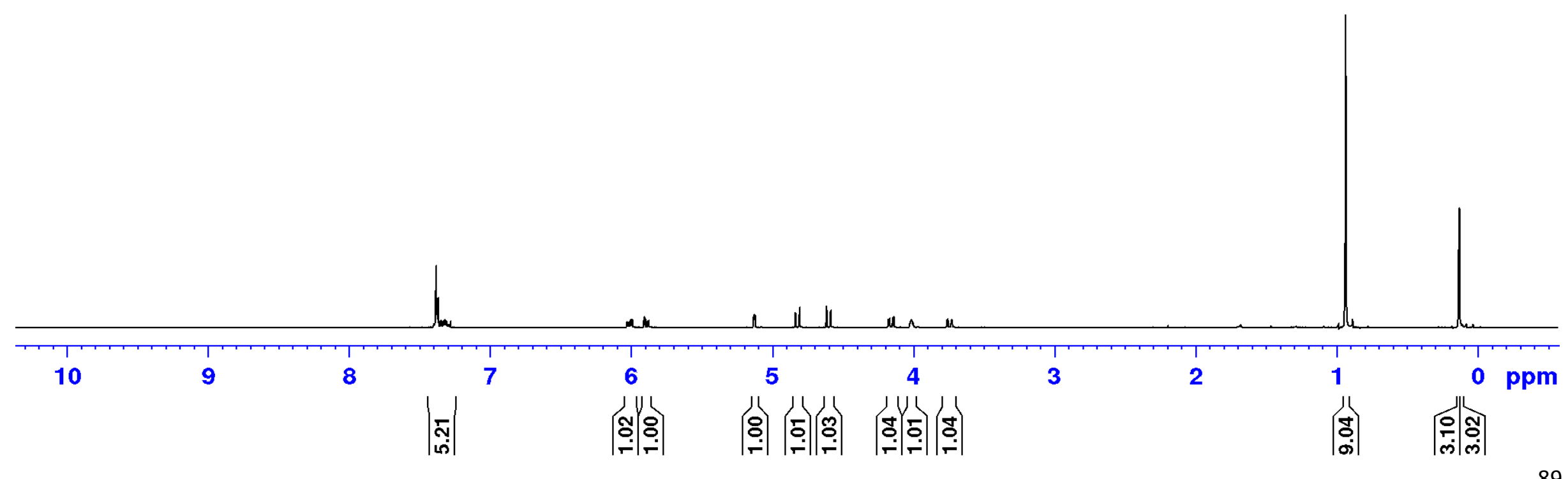




|

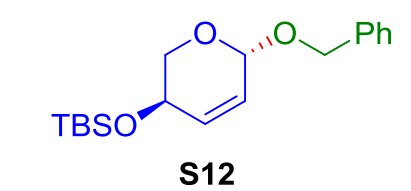

${ }^{13} \mathrm{C}$ NMR (100 MHz, $\mathrm{CDCl}_{3}$ )

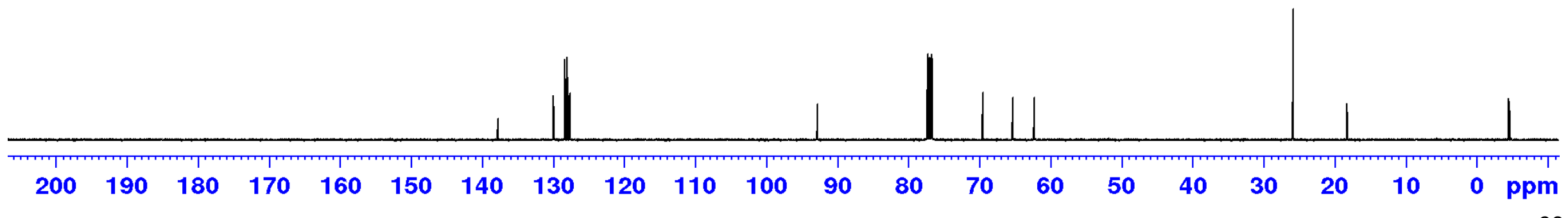




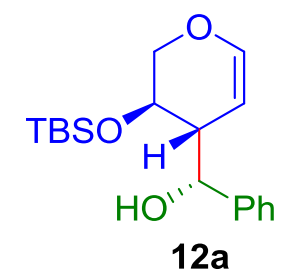

${ }^{1} \mathrm{H}$ NMR (500 MHz, $\mathrm{CDCl}_{3}$ )

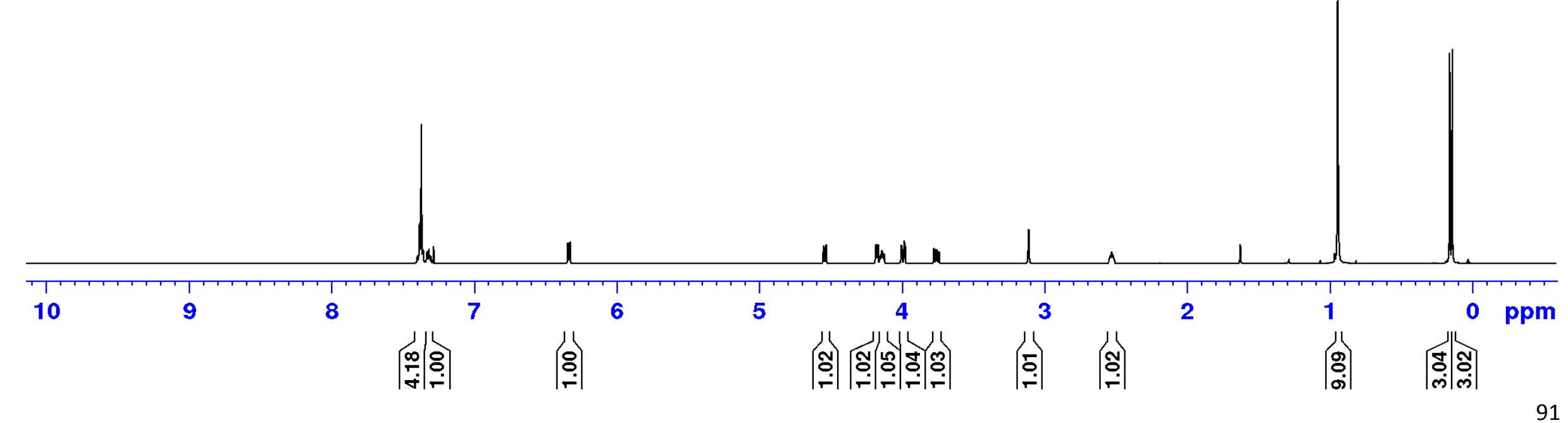




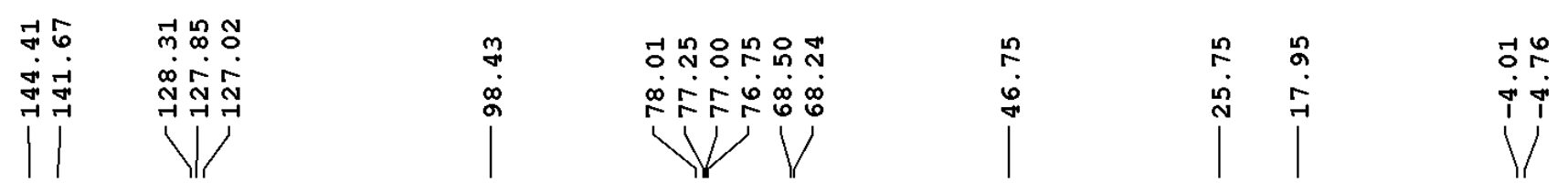

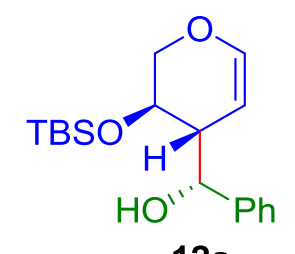

${ }^{13} \mathrm{C}$ NMR (125 MHz, $\mathrm{CDCl}_{3}$ )

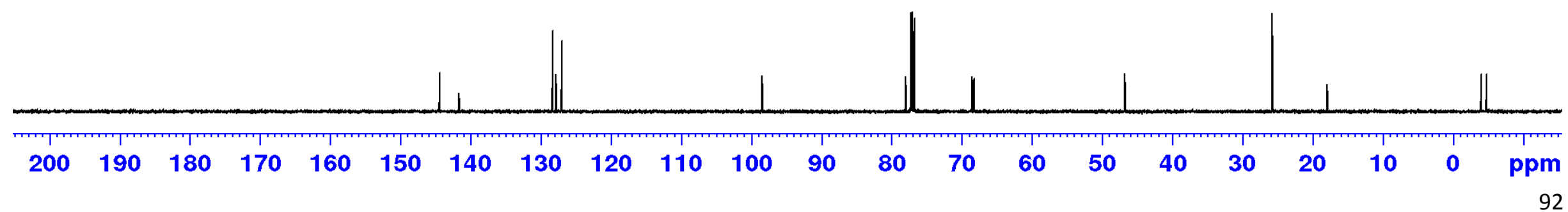




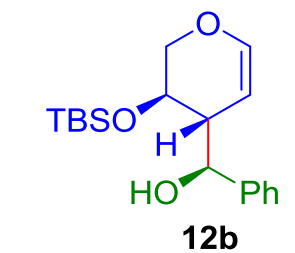

${ }^{1} \mathrm{H}$ NMR $\left(500 \mathrm{MHz}, \mathrm{CDCl}_{3}\right)$

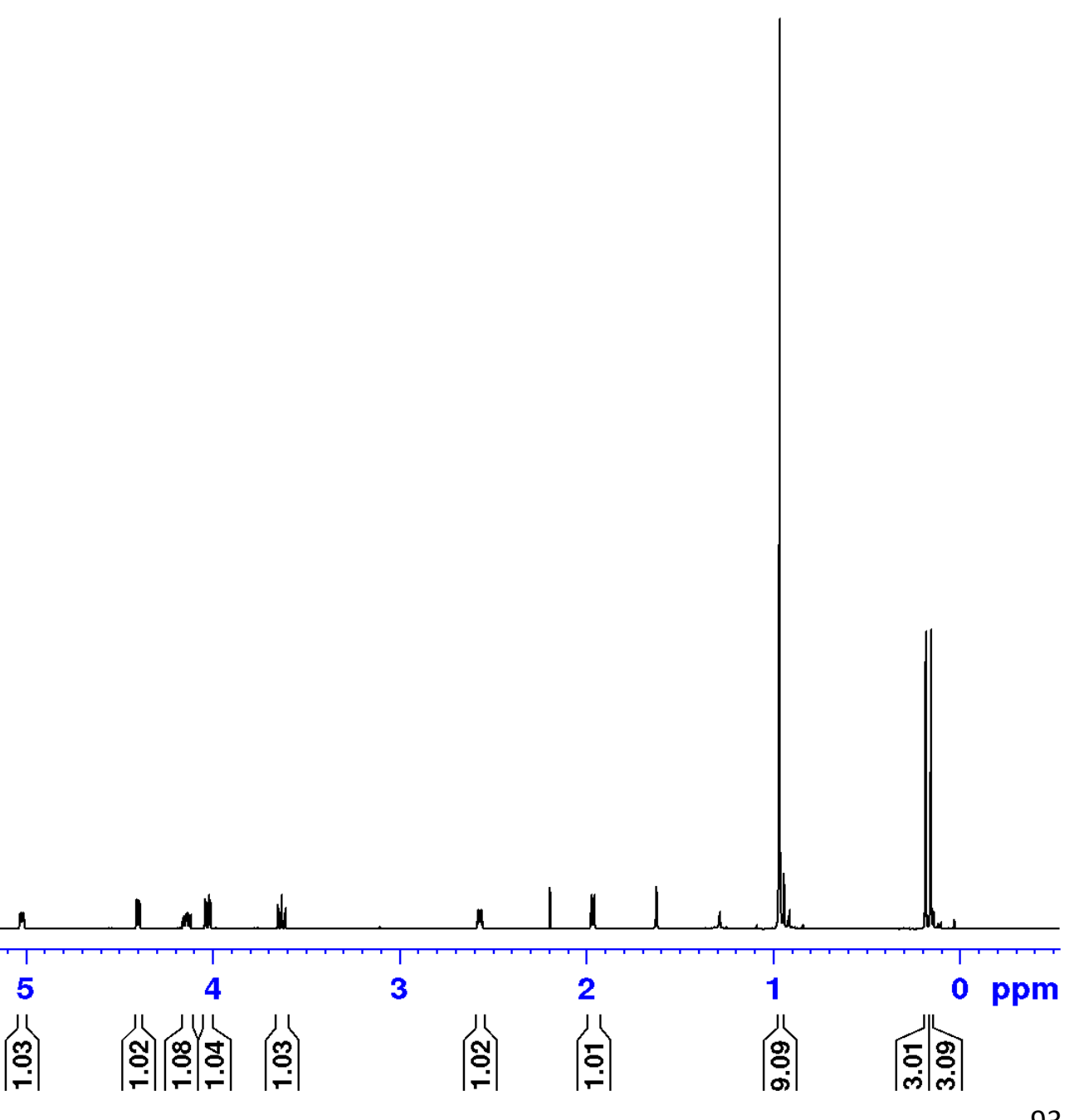




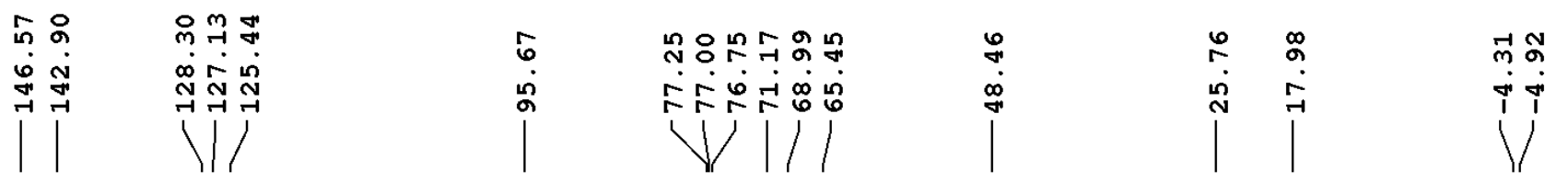

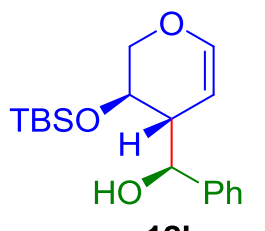

12b

${ }^{13} \mathrm{C}$ NMR (125 MHz, $\mathrm{CDCl}_{3}$ )

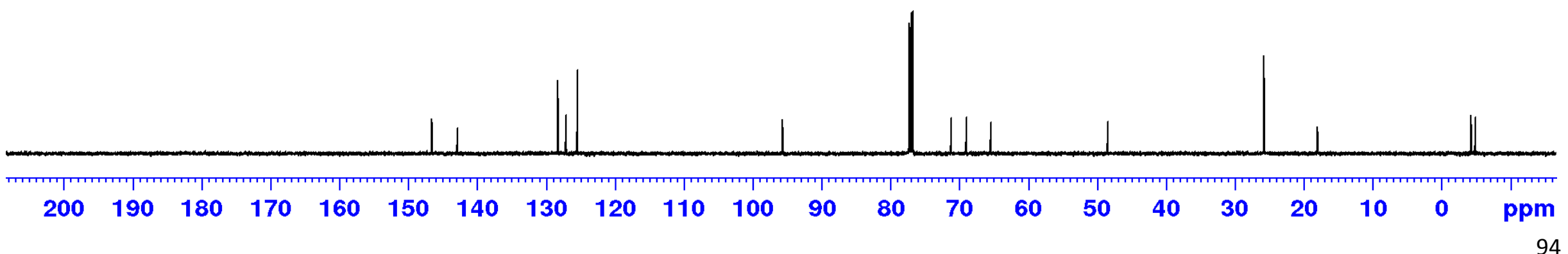




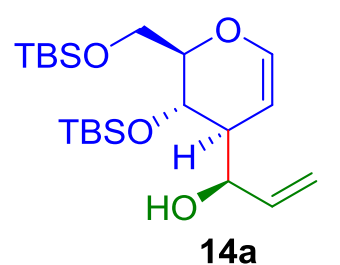

${ }^{1} \mathrm{H}$ NMR (400 MHz, $\mathrm{CDCl}_{3}$ )

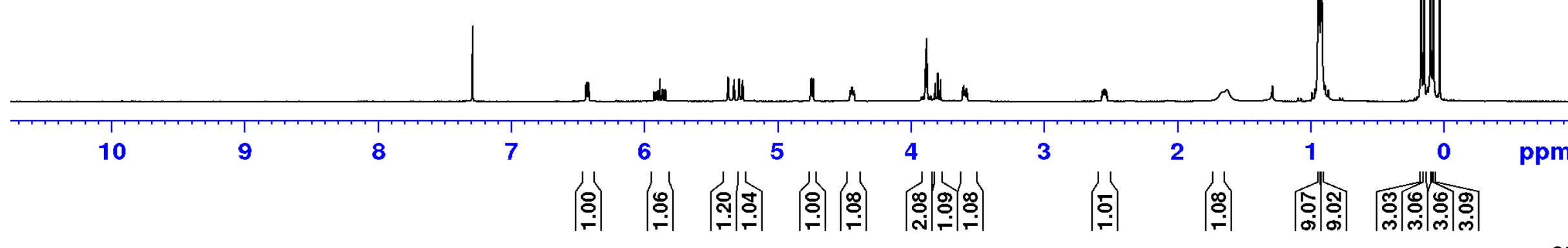




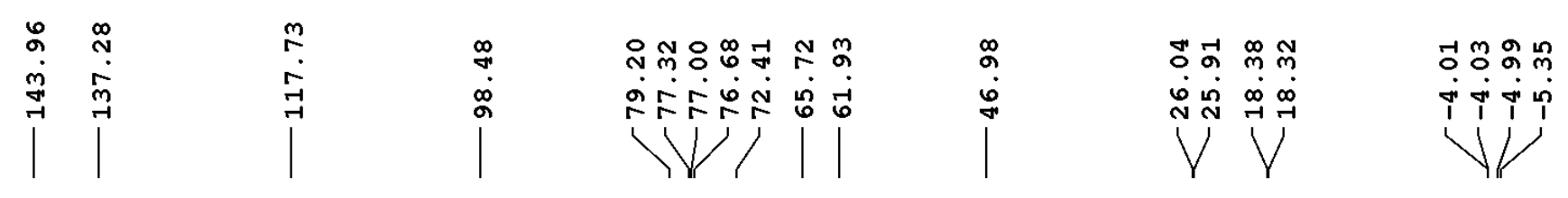

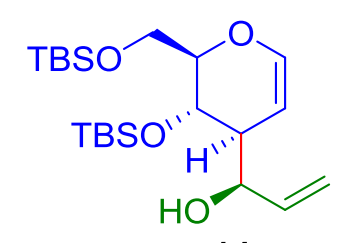

$14 a$

${ }^{13} \mathrm{C}$ NMR (100 MHz, $\mathrm{CDCl}_{3}$ )

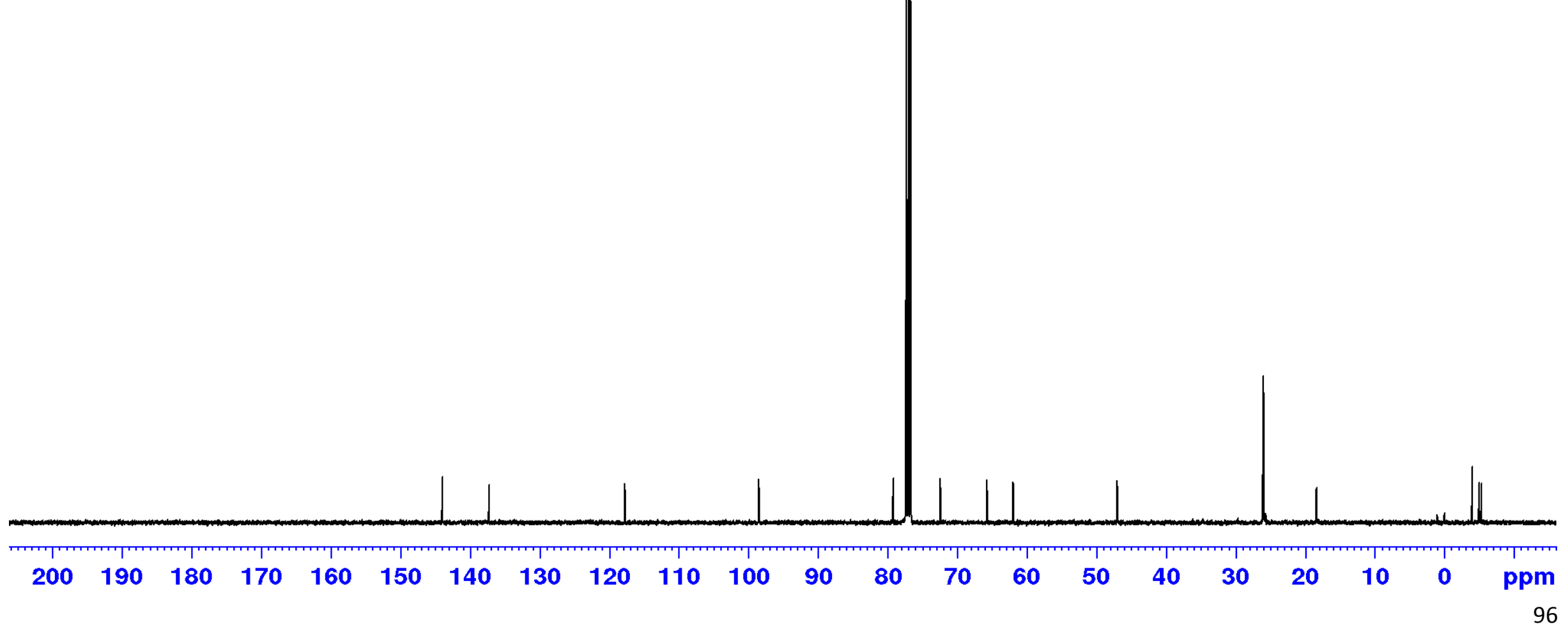




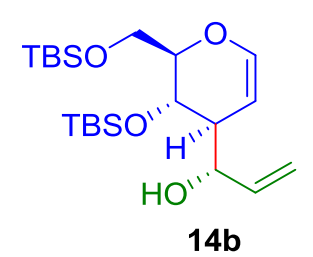

${ }^{1} \mathrm{H}$ NMR (500 MHz, $\mathrm{CDCl}_{3}$ )

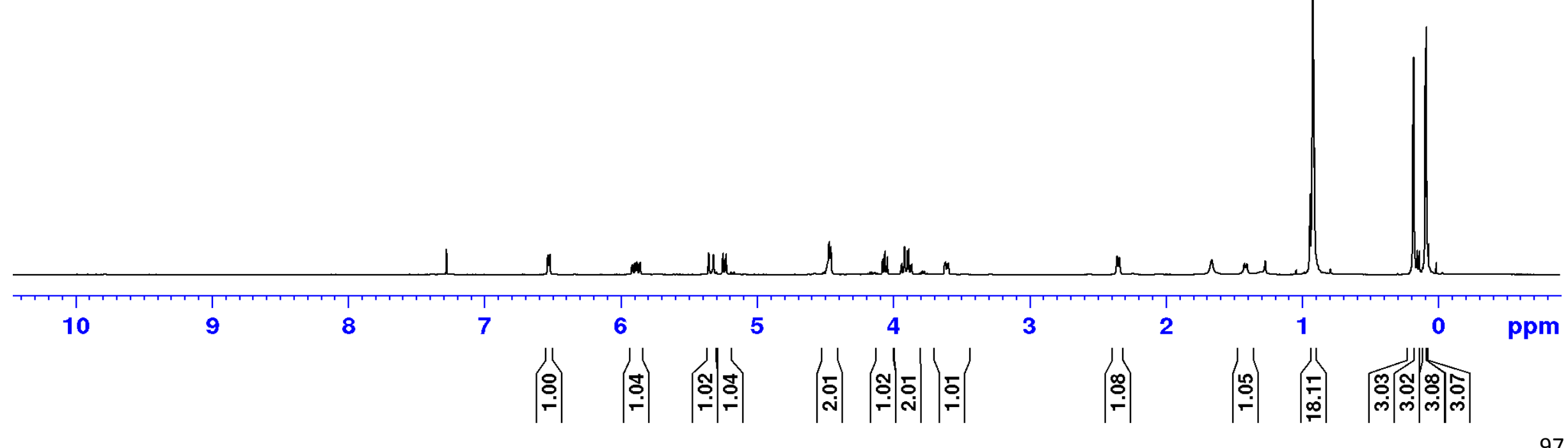




\begin{tabular}{|c|c|c|c|c|c|}
\hline$\stackrel{\infty}{\infty} \stackrel{\infty}{\circ}$ & $\stackrel{n}{n}$ & $\stackrel{\infty}{\infty}$ & ఖู & a & 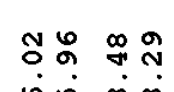 \\
\hline 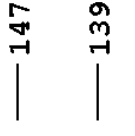 & $\overrightarrow{7}$ & ถั & $\mid \sqrt{ }$ & $\dot{b}$ & 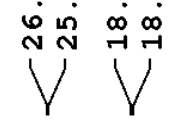 \\
\hline
\end{tabular}

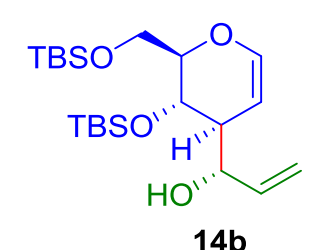

$\left.{ }^{13} \mathrm{C} \mathrm{NMR} \mathrm{(125} \mathrm{MHz,} \mathrm{CDCl}_{3}\right)$

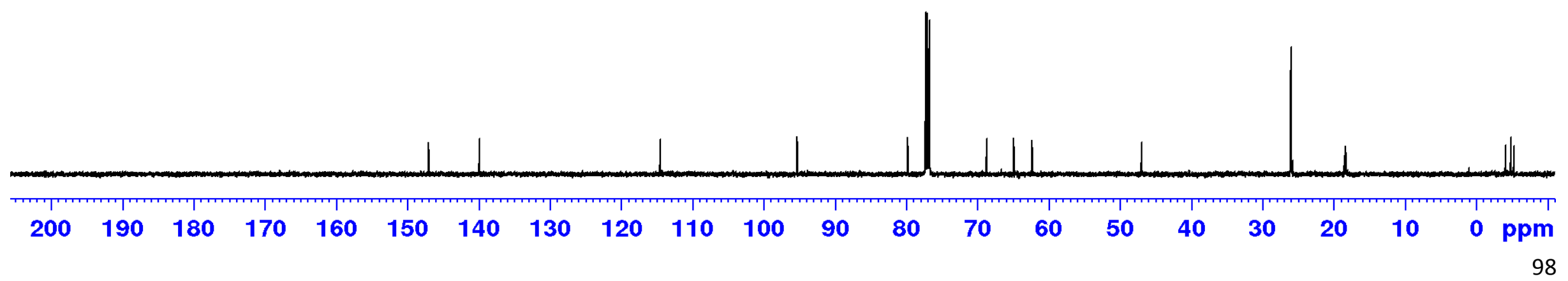




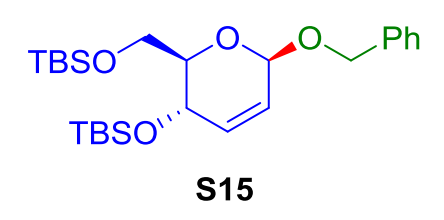

${ }^{1} \mathrm{H}$ NMR (400 MHz, $\mathrm{CDCl}_{3}$ )

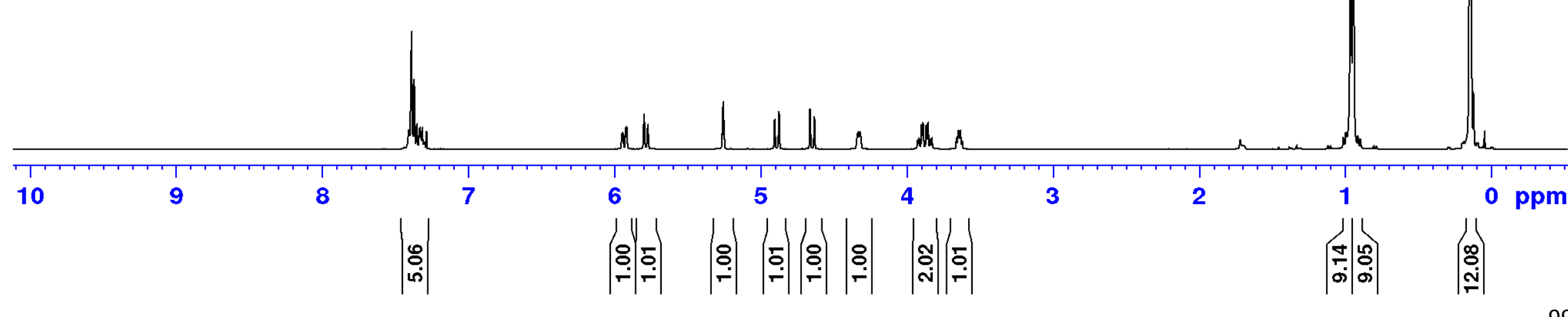




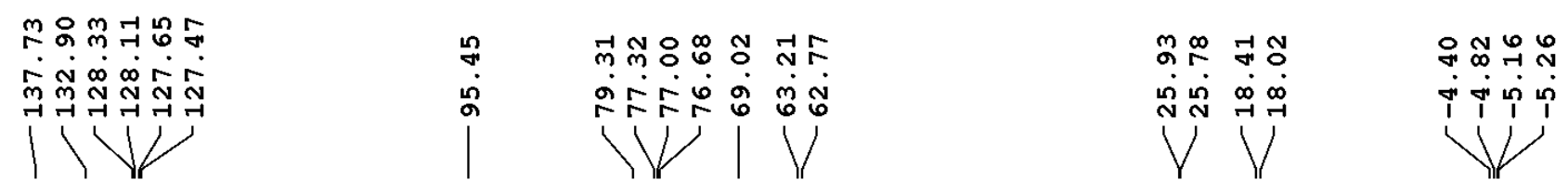

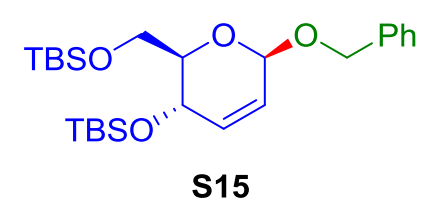

${ }^{13} \mathrm{C}$ NMR (100 MHz, $\mathrm{CDCl}_{3}$ )

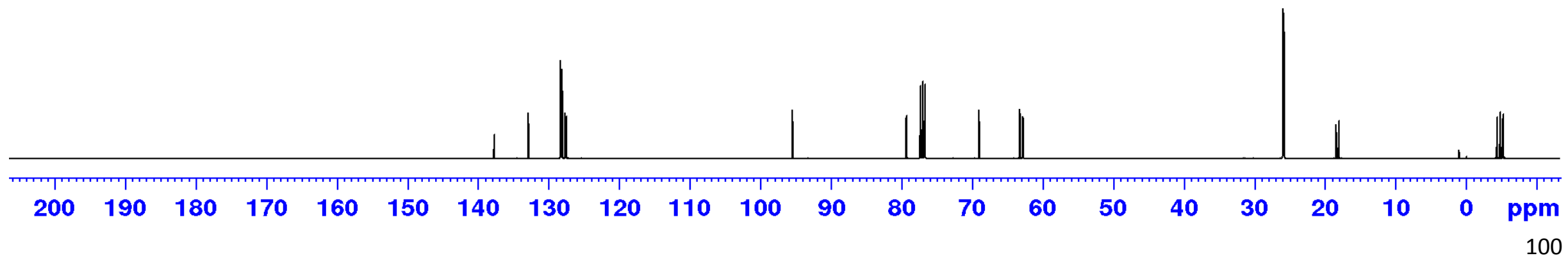




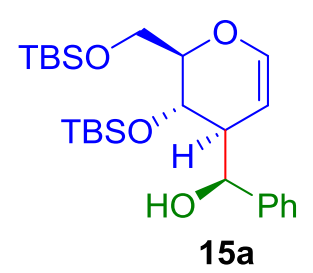

${ }^{1} \mathrm{H}$ NMR (400 MHz, $\mathrm{CDCl}_{3}$ )

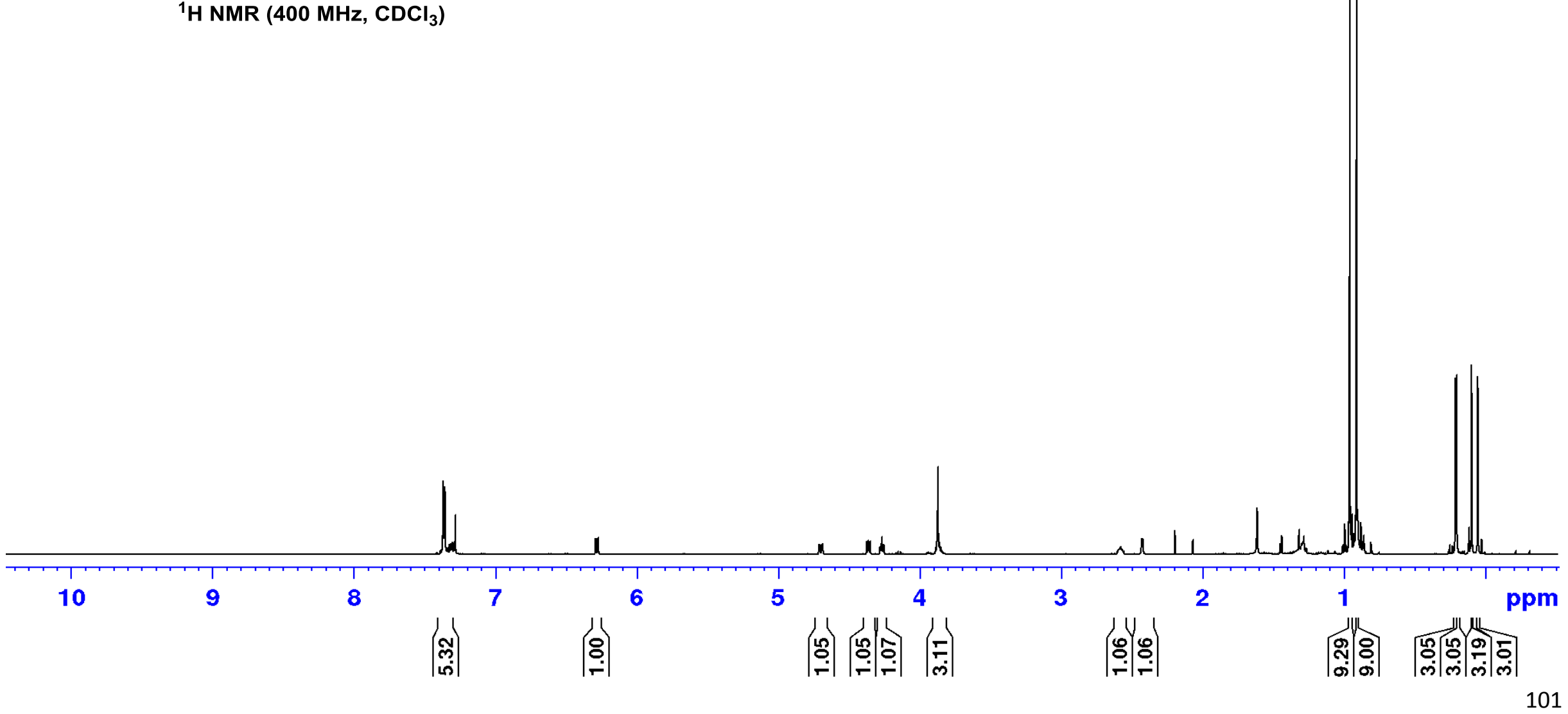




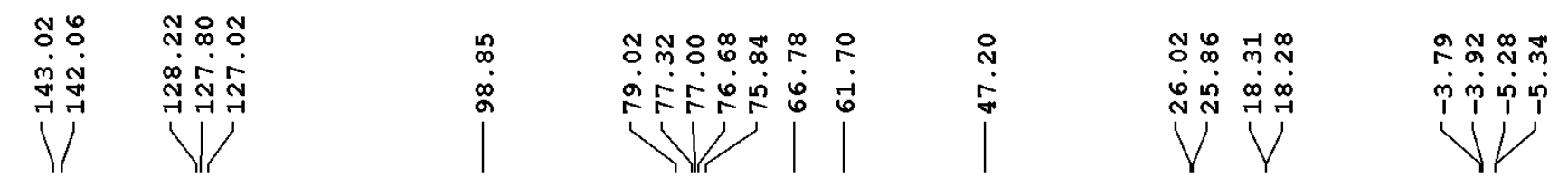

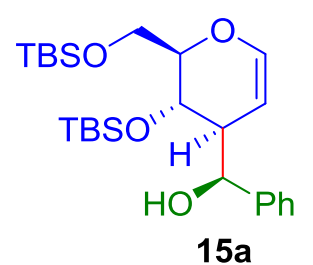

${ }^{13} \mathrm{C}$ NMR (100 MHz, $\mathrm{CDCl}_{3}$ )

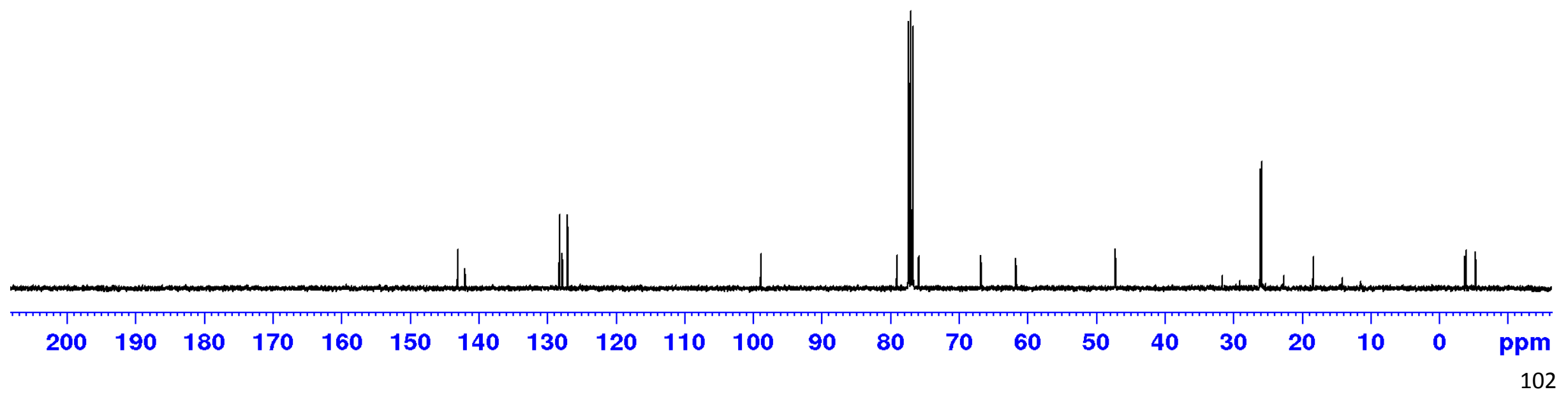




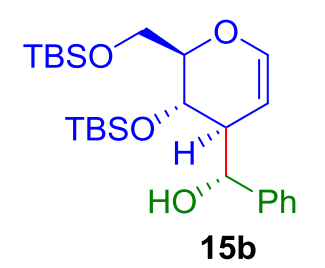

${ }^{1} \mathrm{H}$ NMR (400 MHz, $\mathrm{CDCl}_{3}$ )

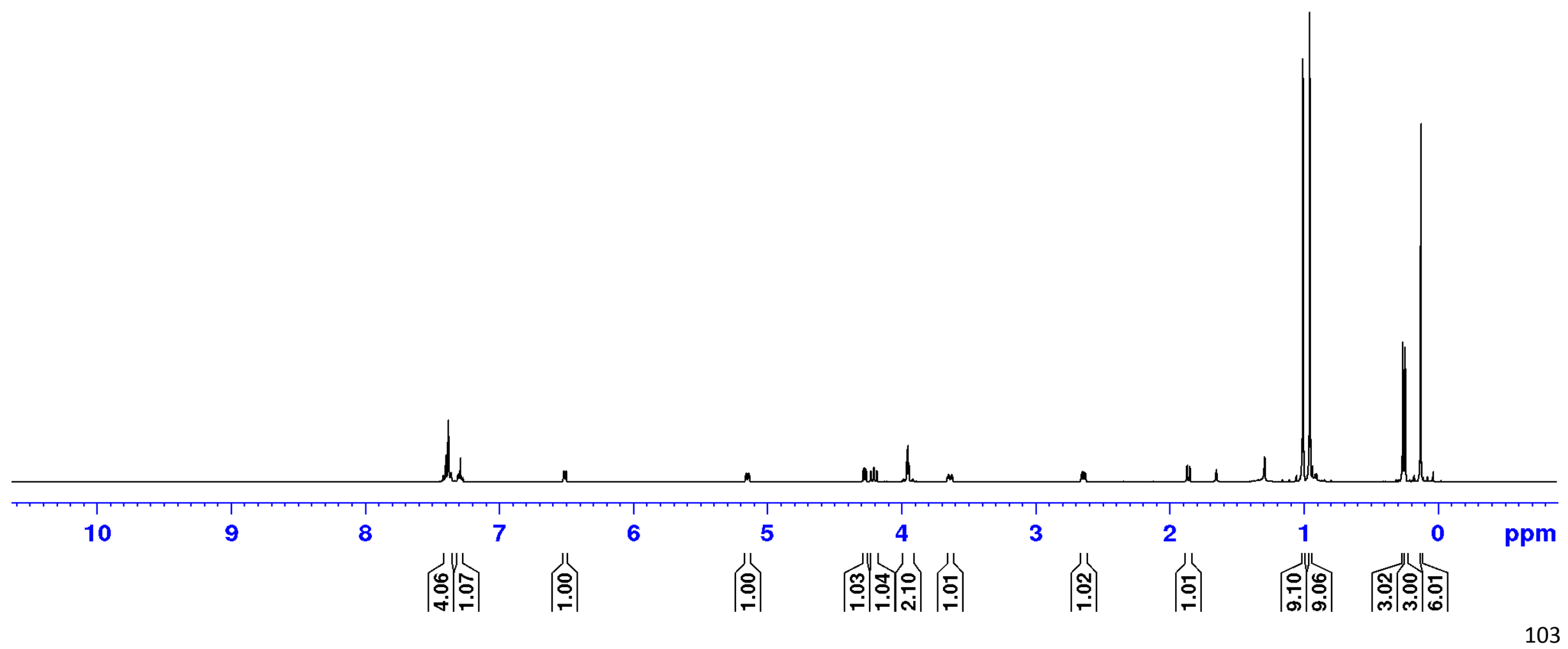




요

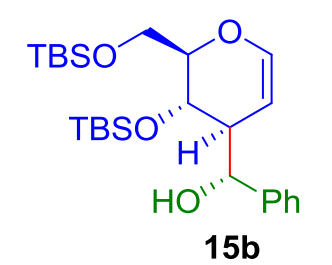

${ }^{13} \mathrm{C}$ NMR (100 MHz, $\mathrm{CDCl}_{3}$ )

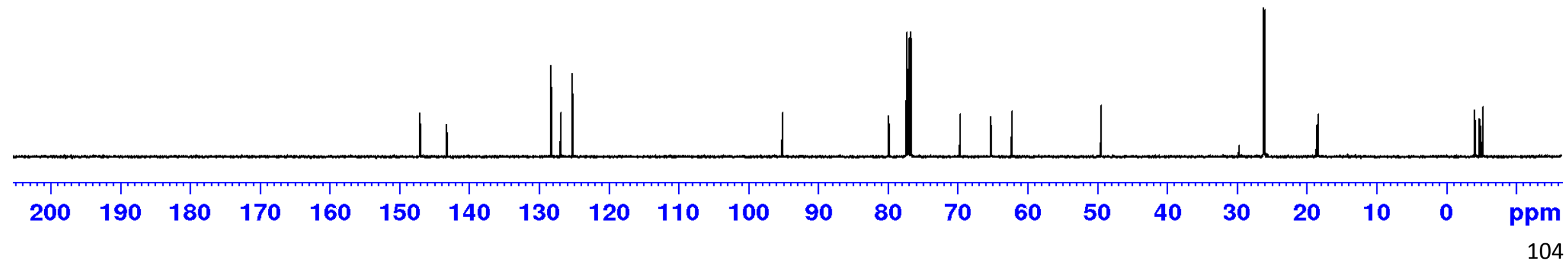



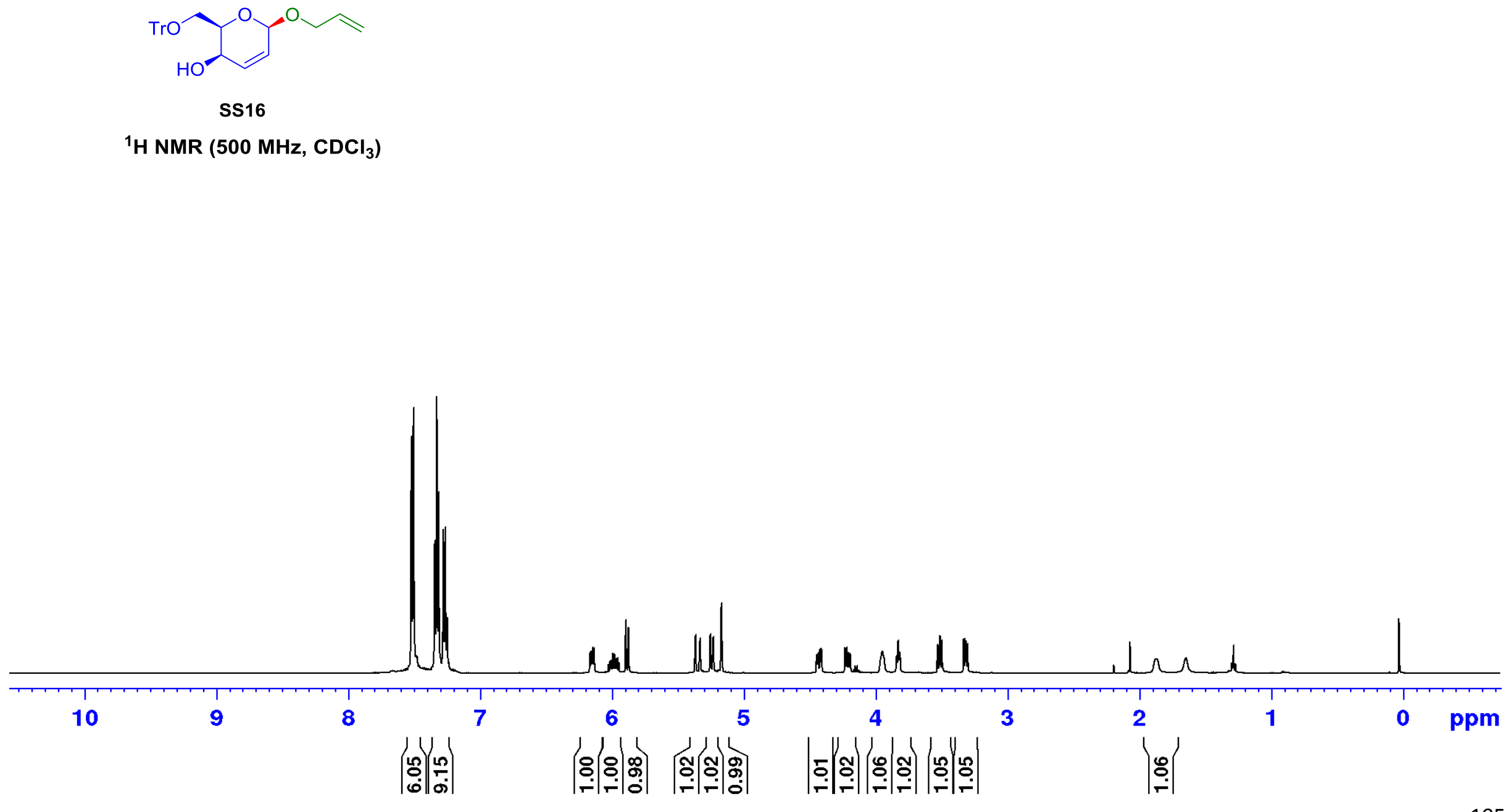

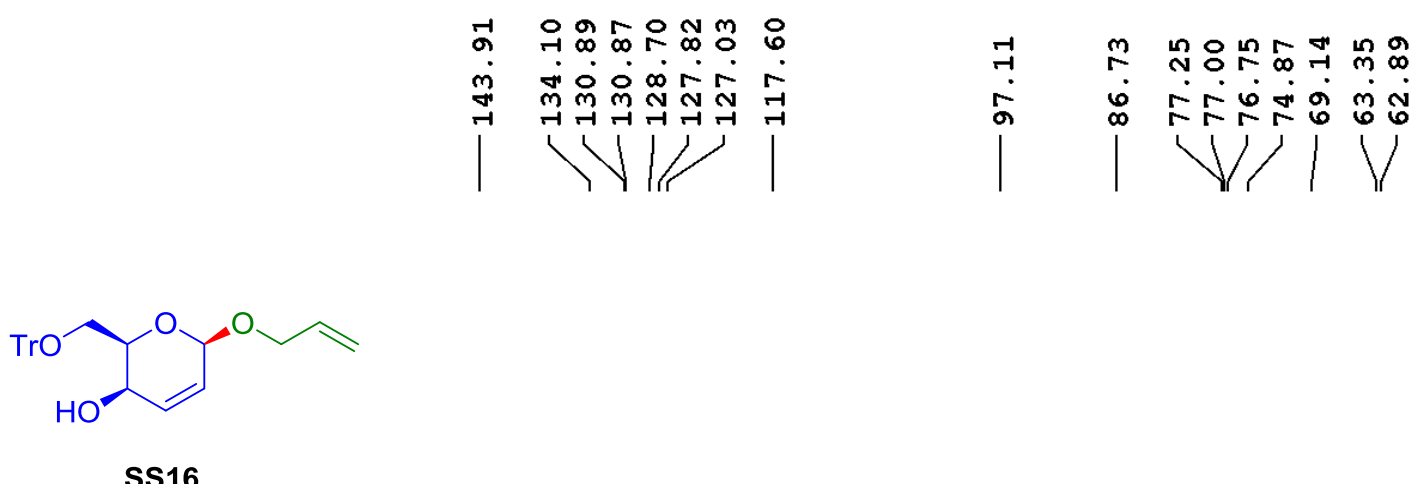

${ }^{13} \mathrm{C}$ NMR (125 MHz, $\mathrm{CDCl}_{3}$ )

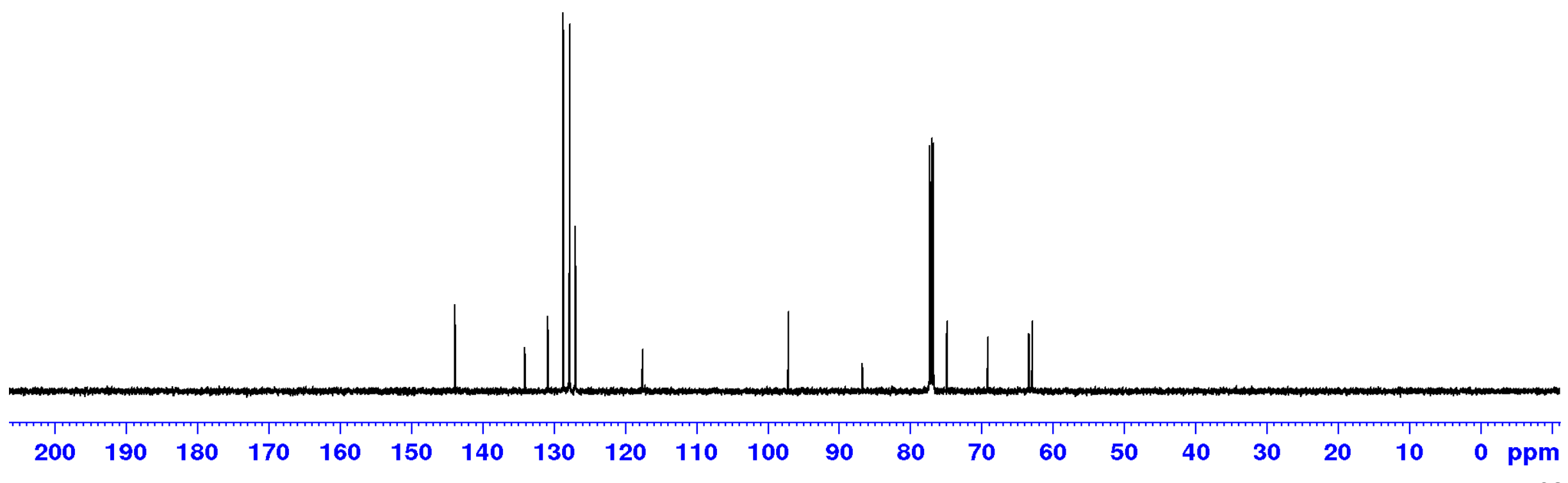




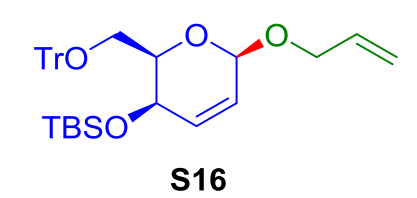

${ }^{1} \mathrm{H}$ NMR (400 MHz, $\mathrm{CDCl}_{3}$ )

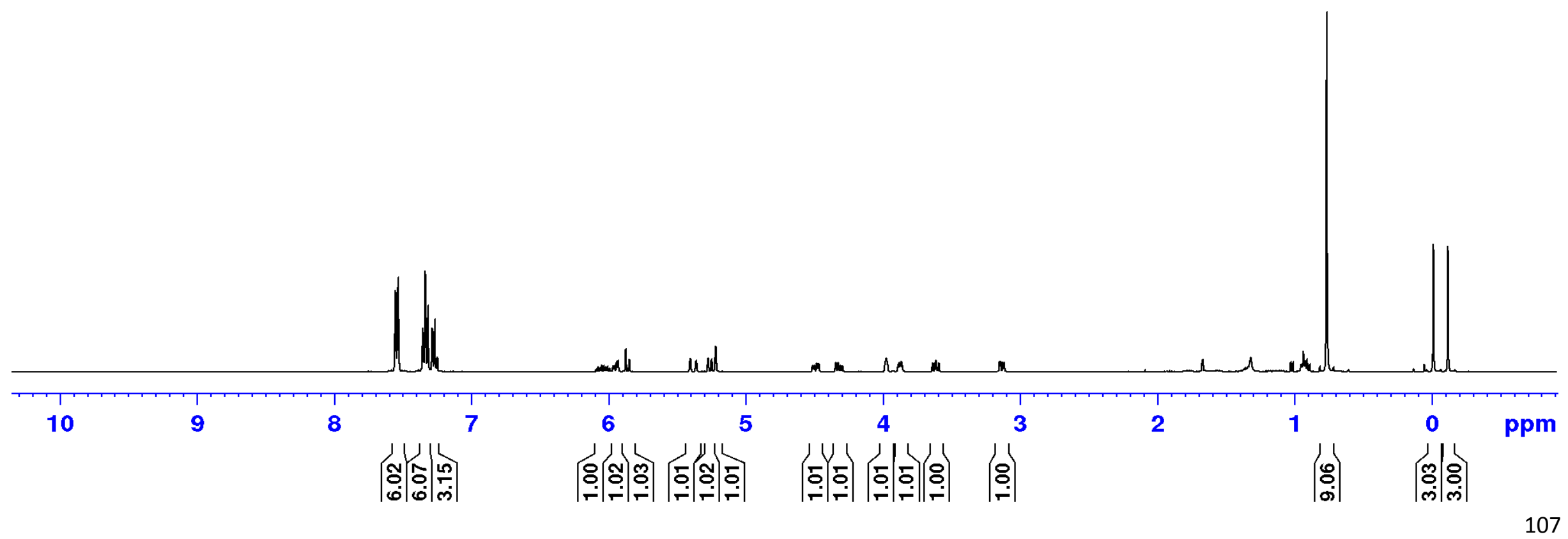



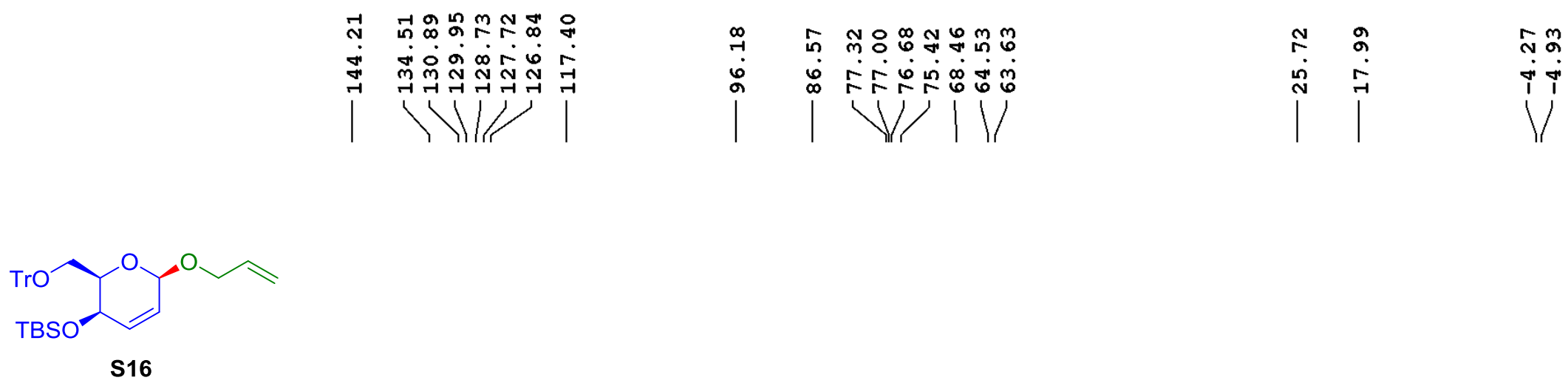

${ }^{13} \mathrm{C}$ NMR (100 MHz, $\mathrm{CDCl}_{3}$ )

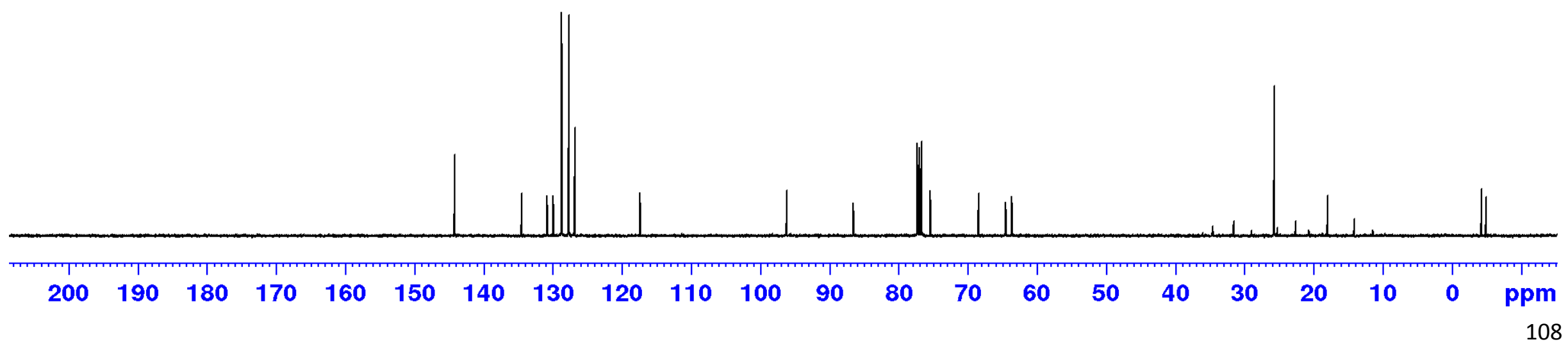




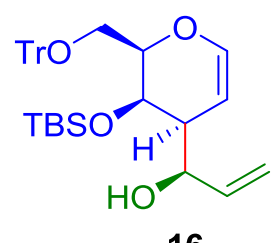

${ }^{1} \mathrm{H}$ NMR (500 MHz, $\mathrm{CDCl}_{3}$ )

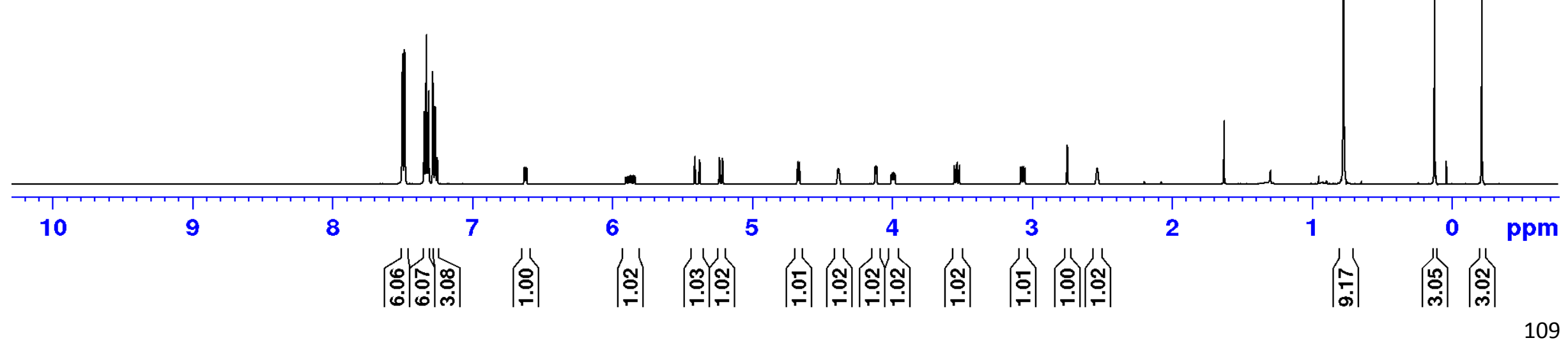




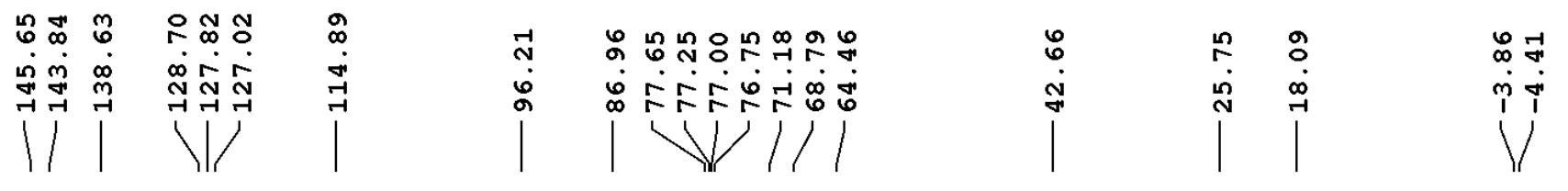

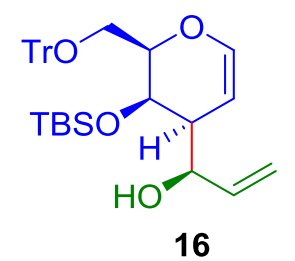

${ }^{13} \mathrm{C}$ NMR (125 MHz, $\mathrm{CDCl}_{3}$ )

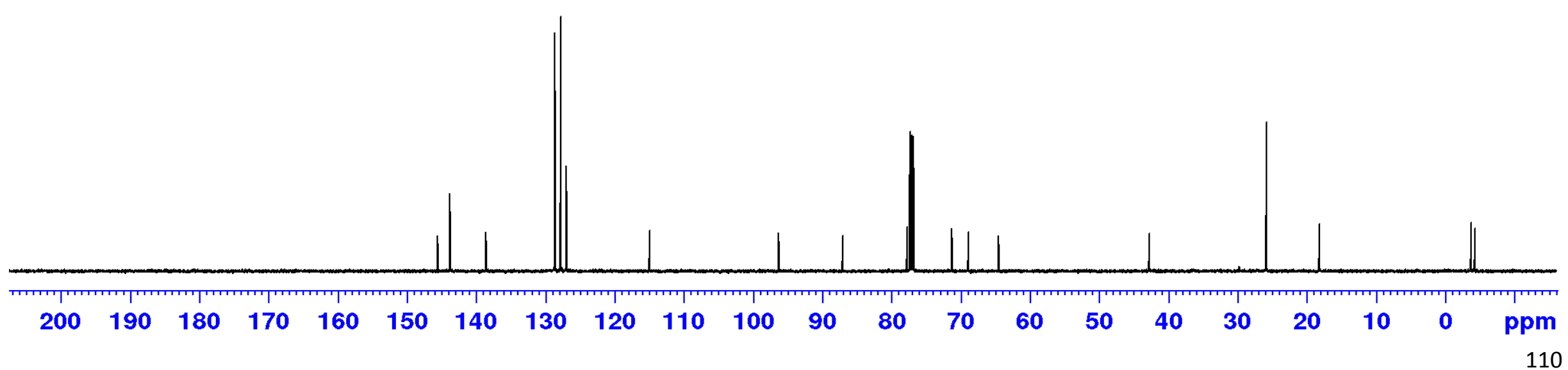




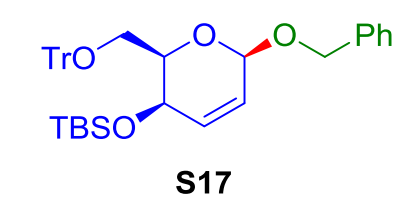

${ }^{1} \mathrm{H}$ NMR (400 MHz, $\mathrm{CDCl}_{3}$ )

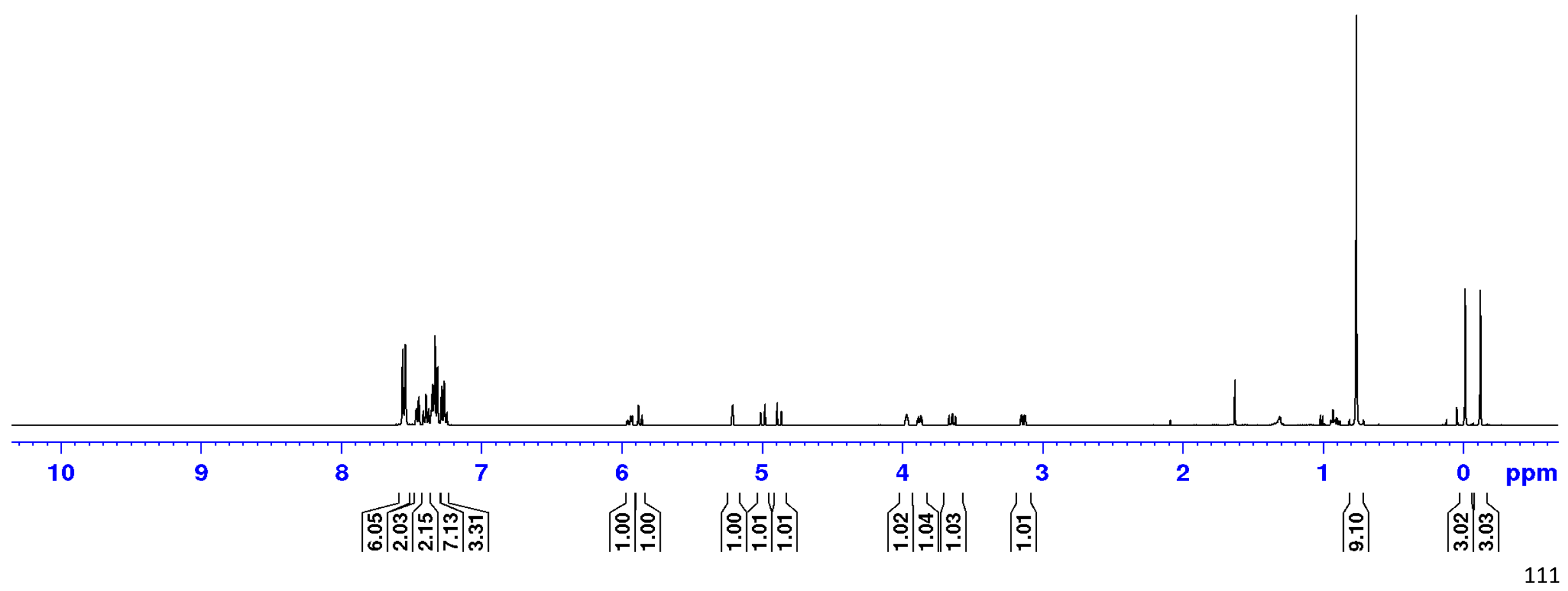




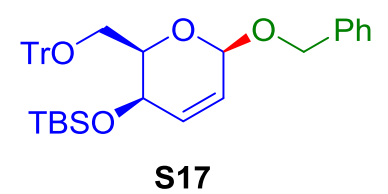

${ }^{13} \mathrm{C}$ NMR (100 MHz, $\mathrm{CDCl}_{3}$ )

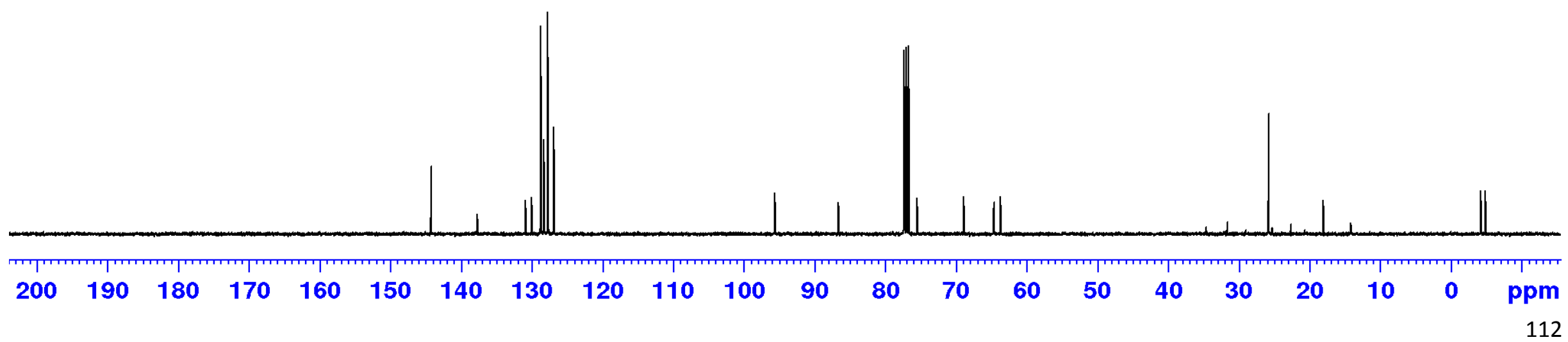




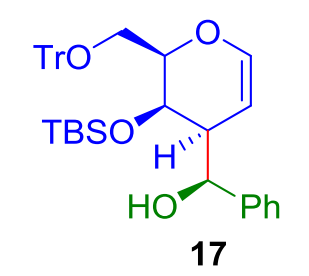

${ }^{1} \mathrm{H}$ NMR (500 MHz, $\mathrm{CDCl}_{3}$ )

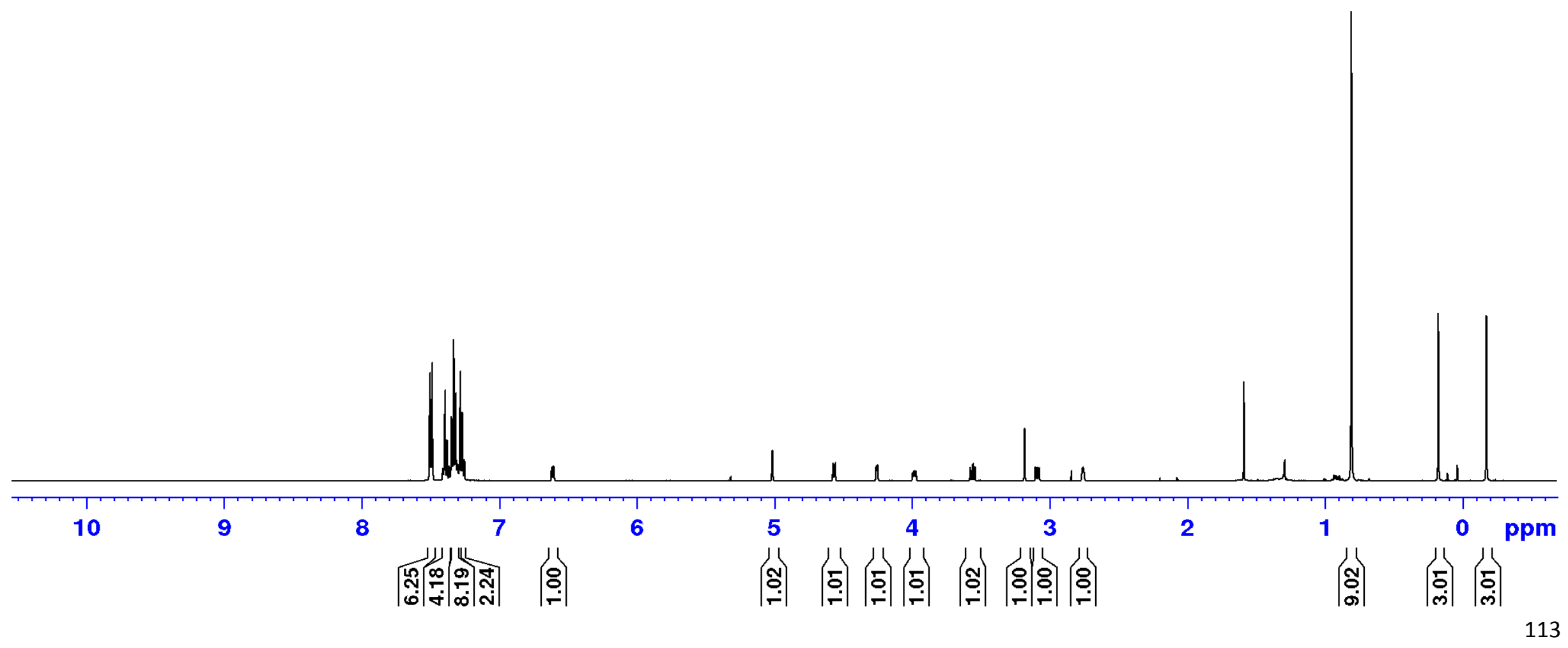



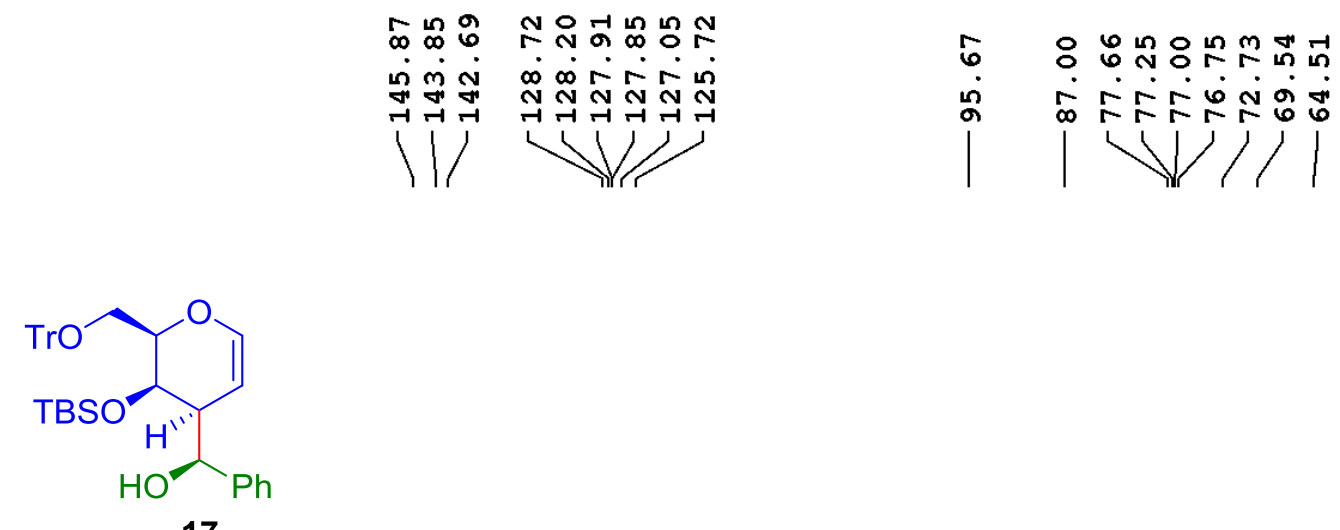

17

${ }^{13} \mathrm{C}$ NMR (125 MHz, $\mathrm{CDCl}_{3}$ )

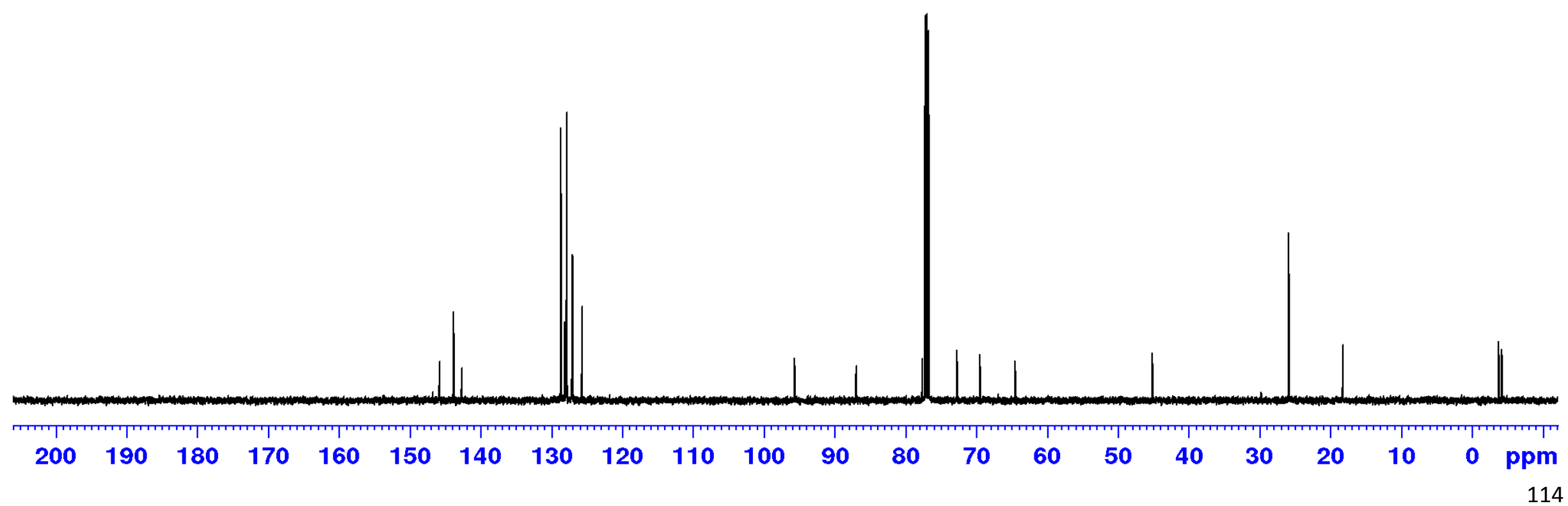




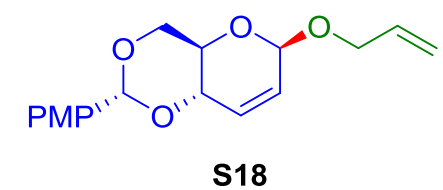

${ }^{1} \mathrm{H}$ NMR (500 MHz, $\mathrm{CDCl}_{3}$ )

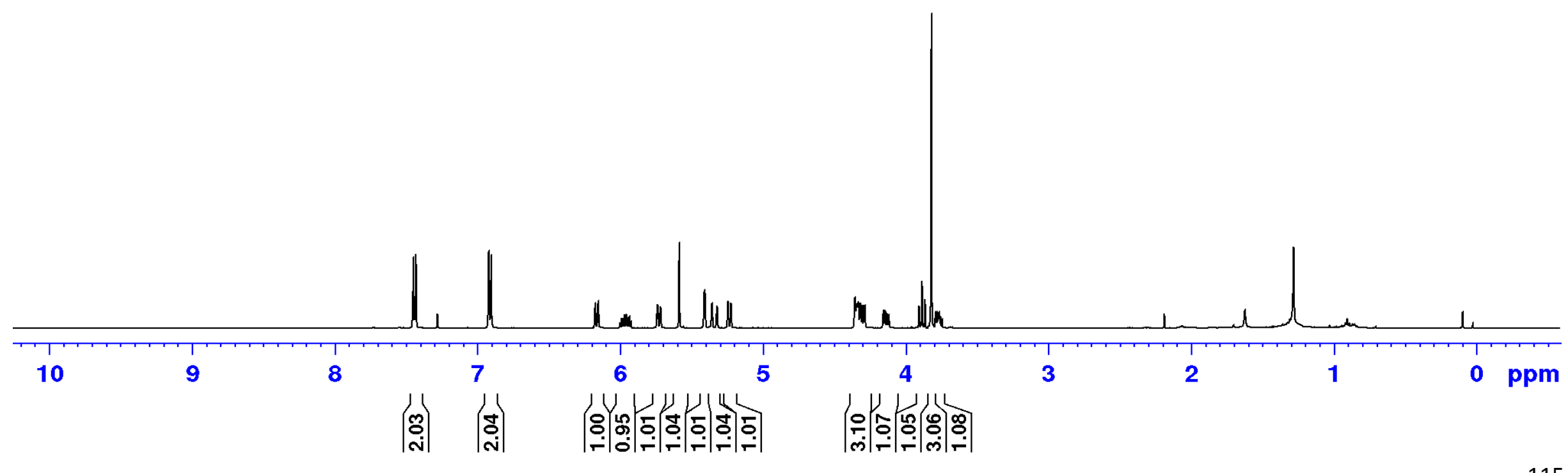




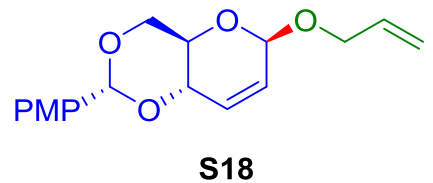

${ }^{13} \mathrm{C}$ NMR (125 MHz, $\left.\mathrm{CDCl}_{3}\right)$

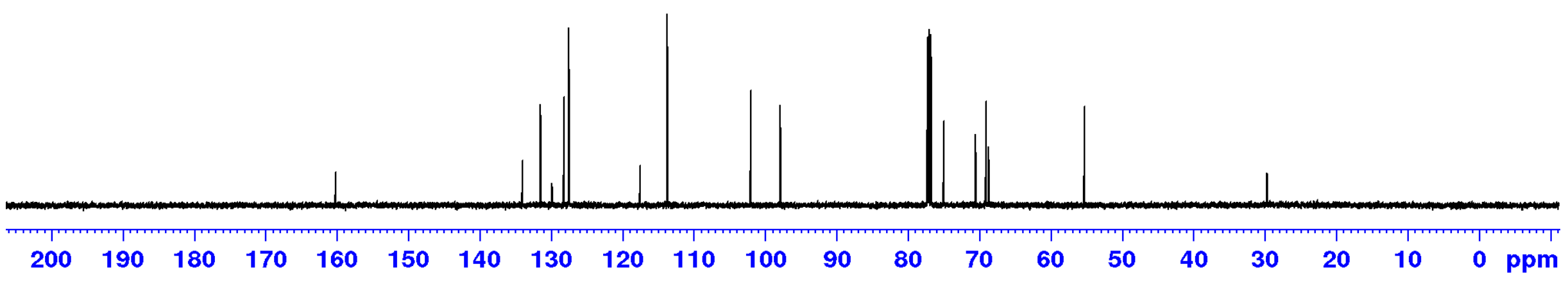




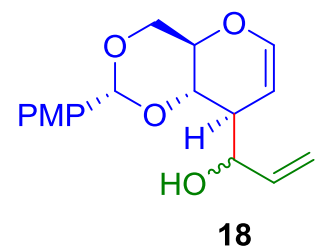

${ }^{1} \mathrm{H}$ NMR (400 MHz, $\mathrm{CDCl}_{3}$ )

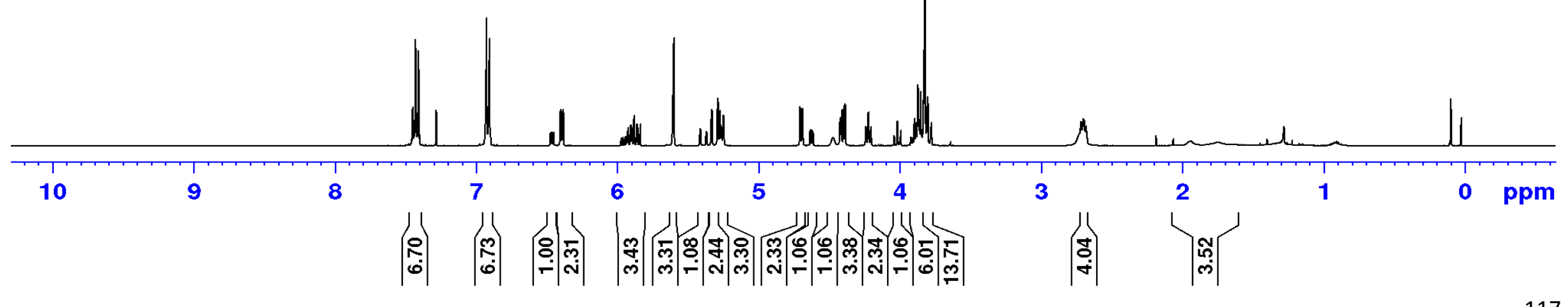




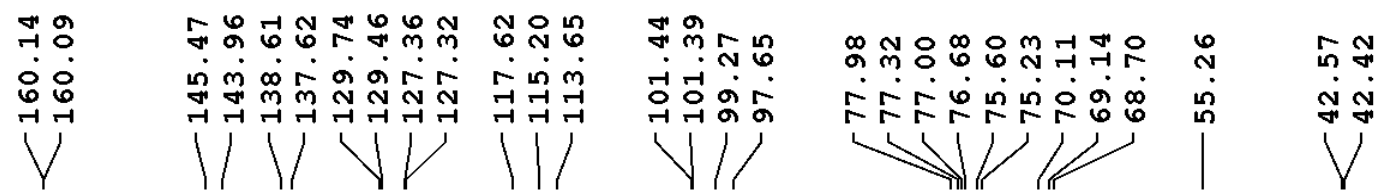

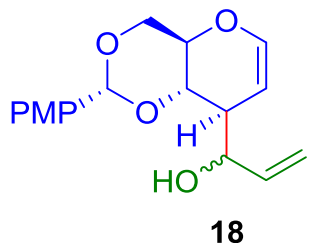

${ }^{13} \mathrm{C}$ NMR $\left(100 \mathrm{MHz}, \mathrm{CDCl}_{3}\right)$

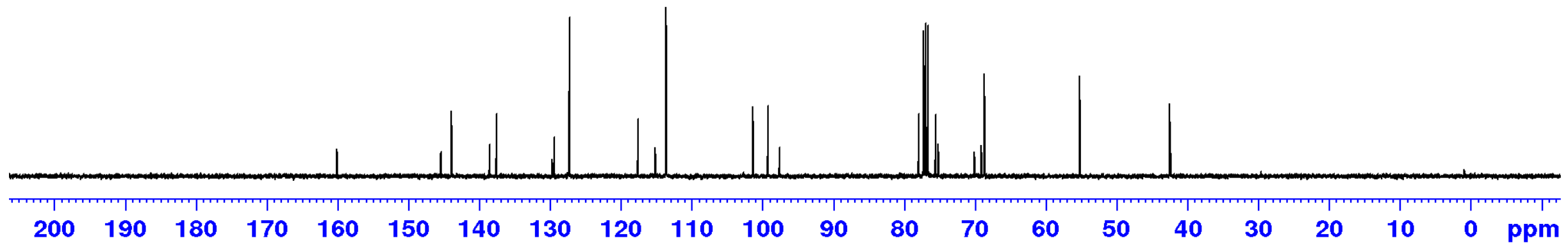




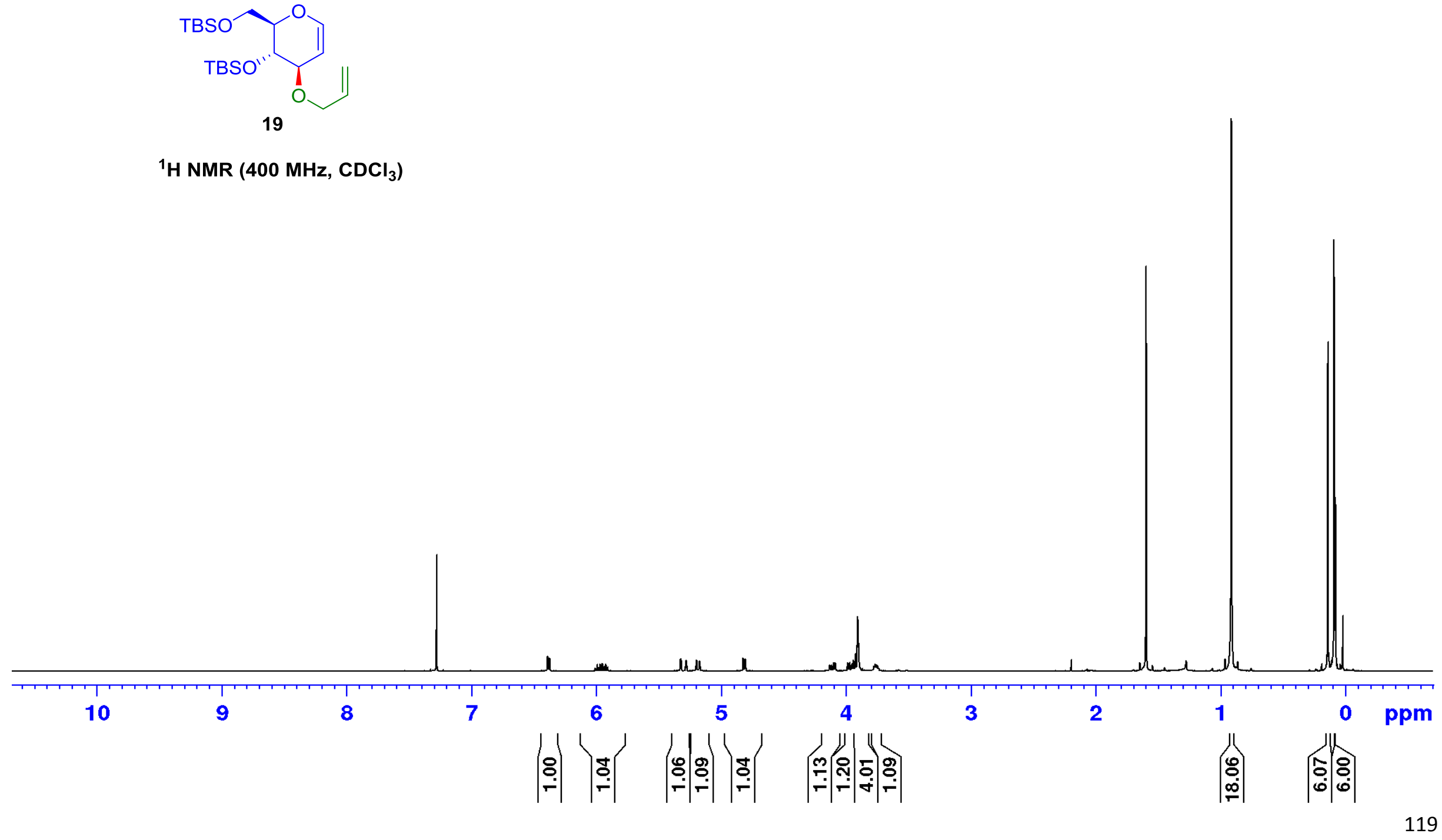




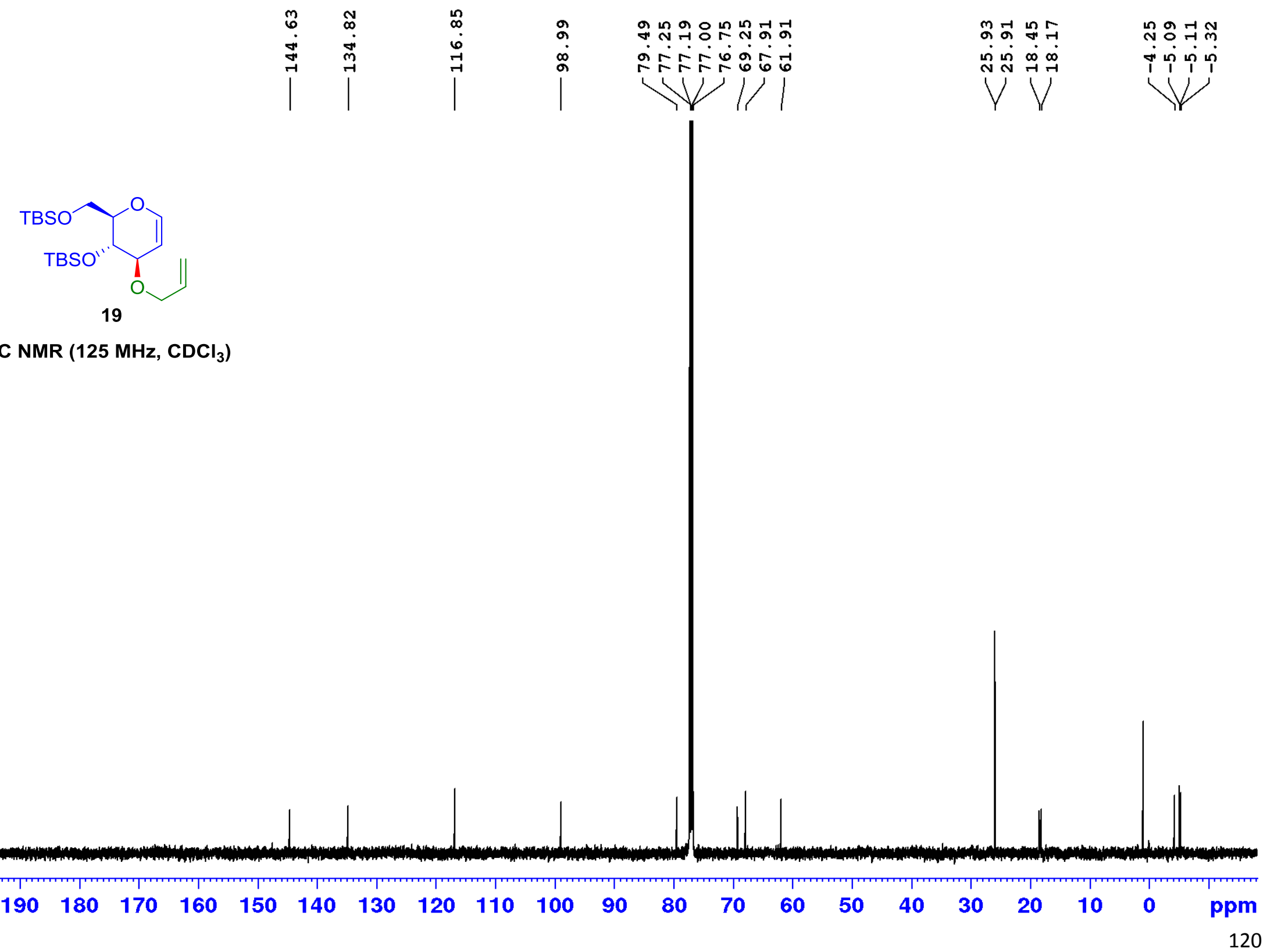




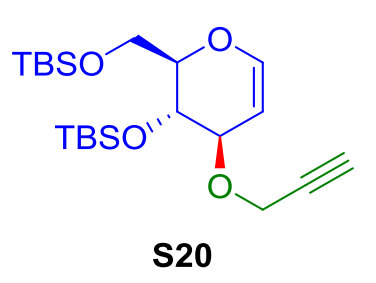

${ }^{1} \mathrm{H}$ NMR (400 MHz, $\mathrm{CDCl}_{3}$ )

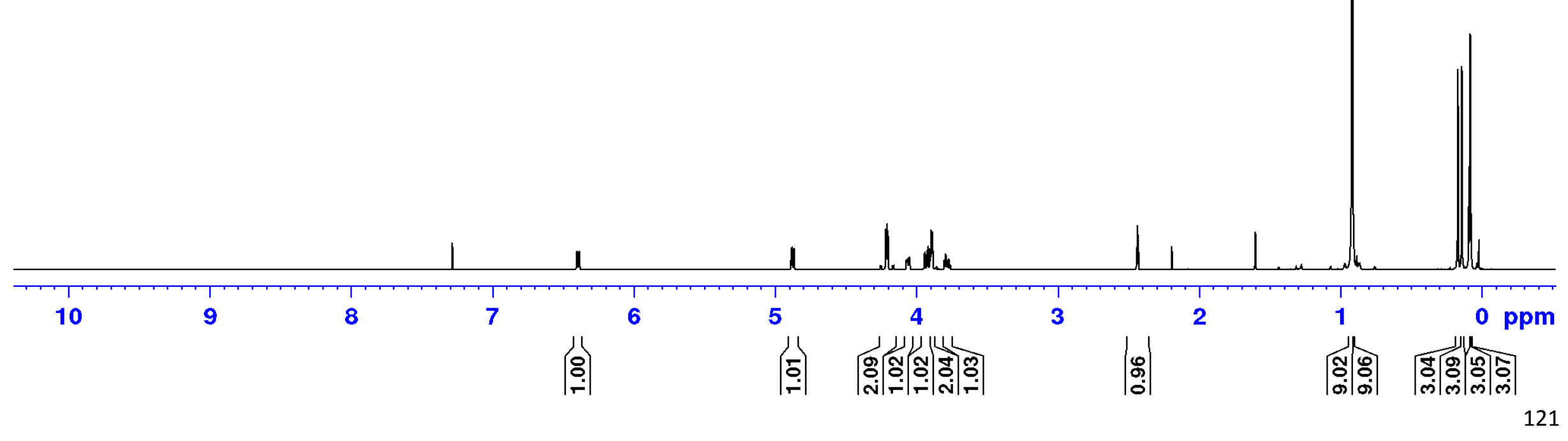



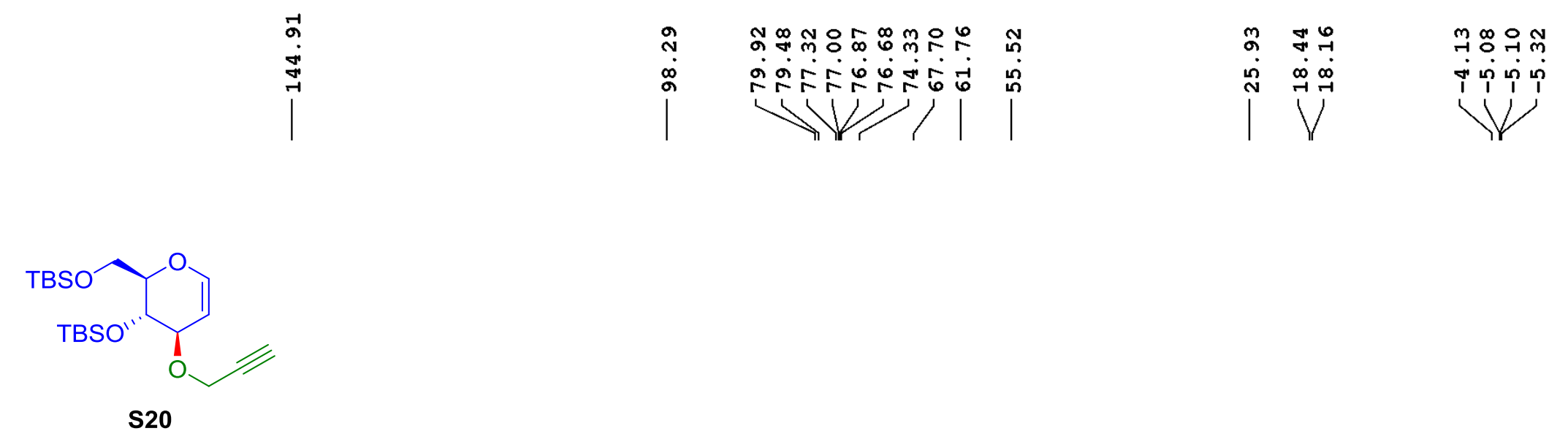

${ }^{13} \mathrm{C}$ NMR (100 MHz, $\mathrm{CDCl}_{3}$ )

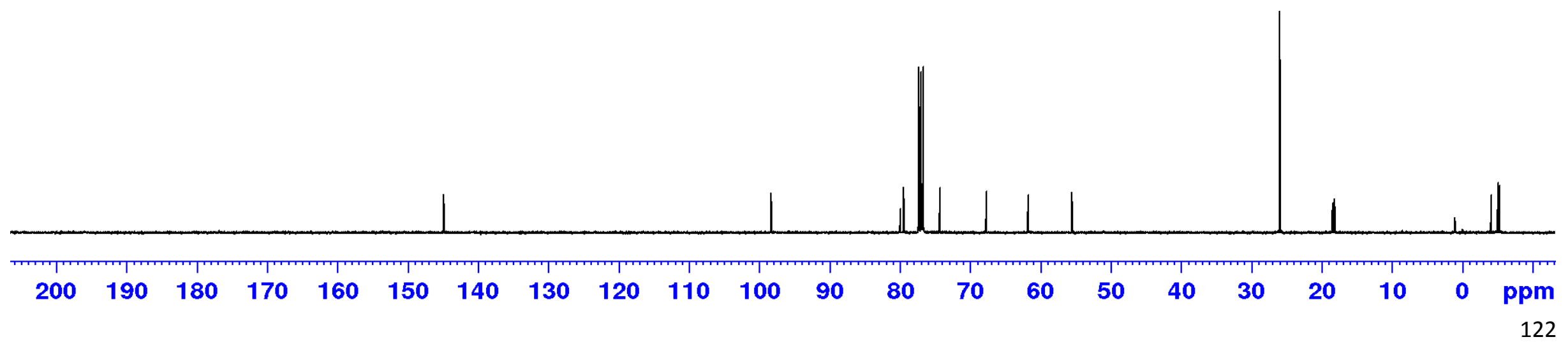




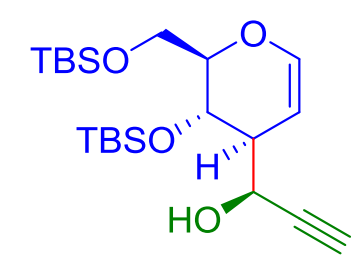

20

${ }^{1} \mathrm{H}$ NMR (500 MHz, $\mathrm{CDCl}_{3}$ )

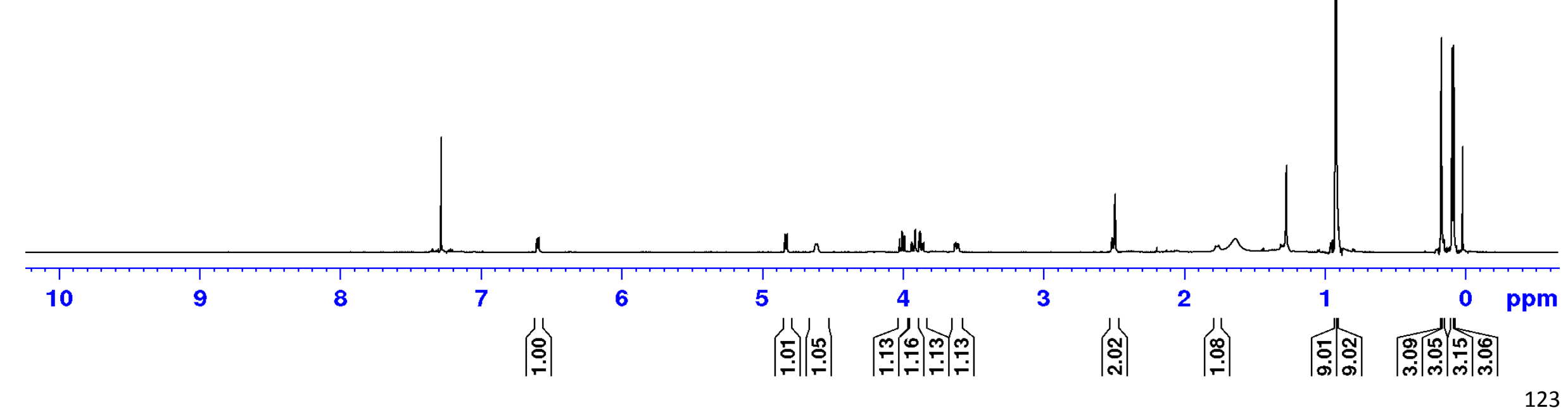




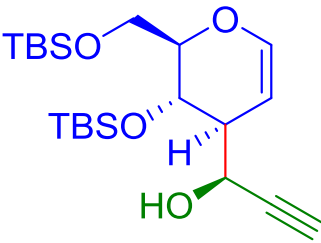

20

${ }^{13} \mathrm{C}$ NMR (125 MHz, $\left.\mathrm{CDCl}_{3}\right)$

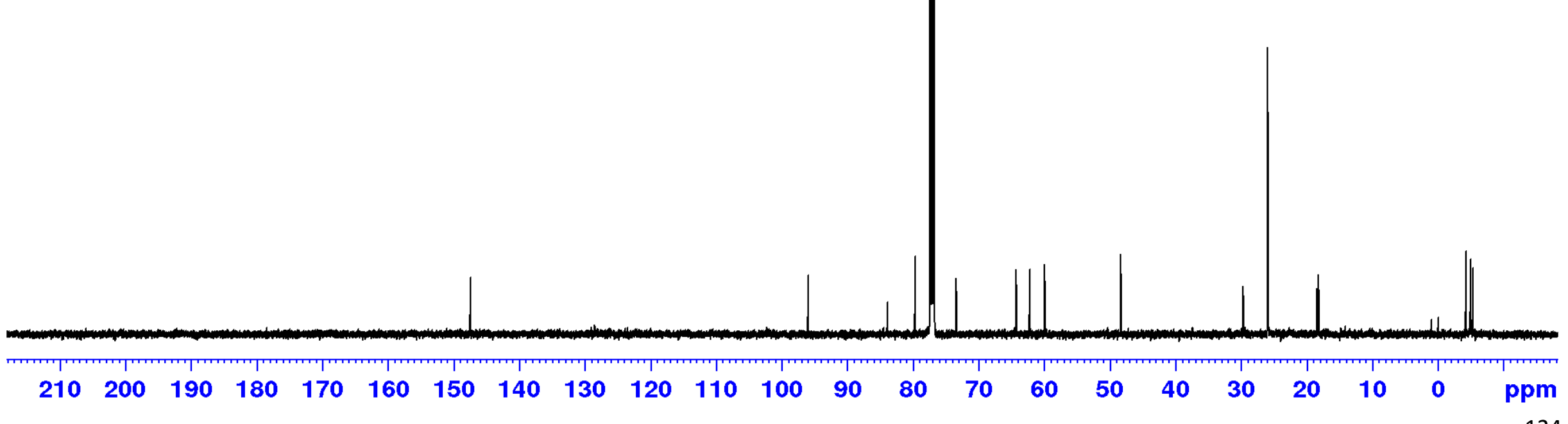




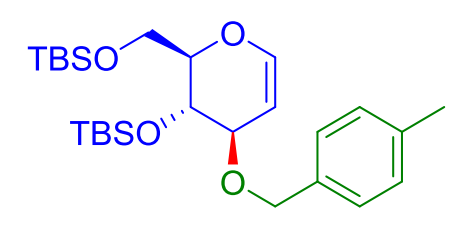

S21

${ }^{1} \mathrm{H}$ NMR (500 MHz, $\mathrm{CDCl}_{3}$ )

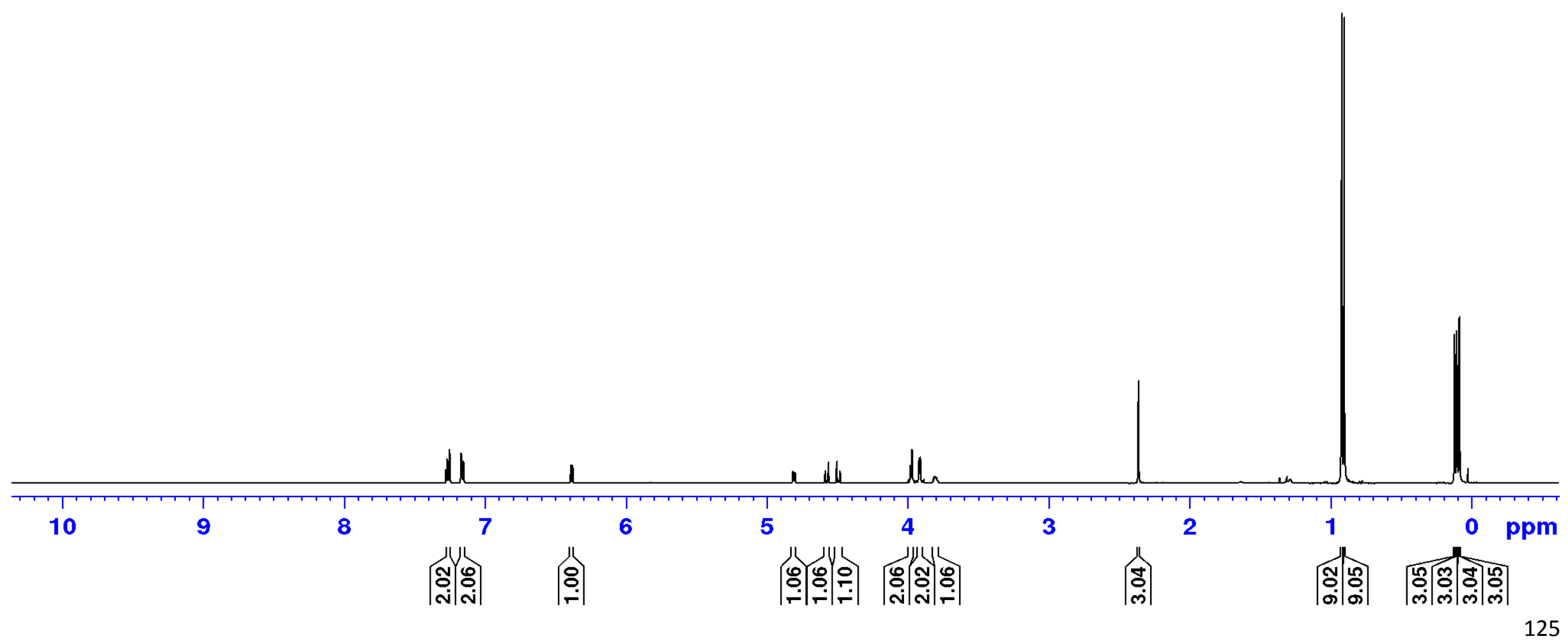




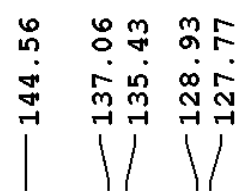

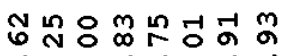

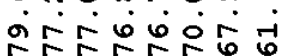

ถํํำ늄ำ

ผู่

VIV

옥ㄴㅇㅇㅇㅜ 운

WII

1

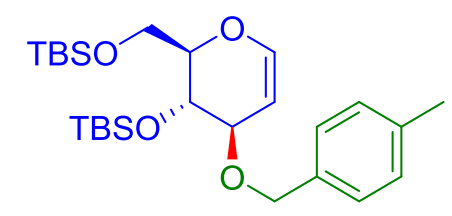

S21

${ }^{13} \mathrm{C}$ NMR (125 MHz, $\mathrm{CDCl}_{3}$ )

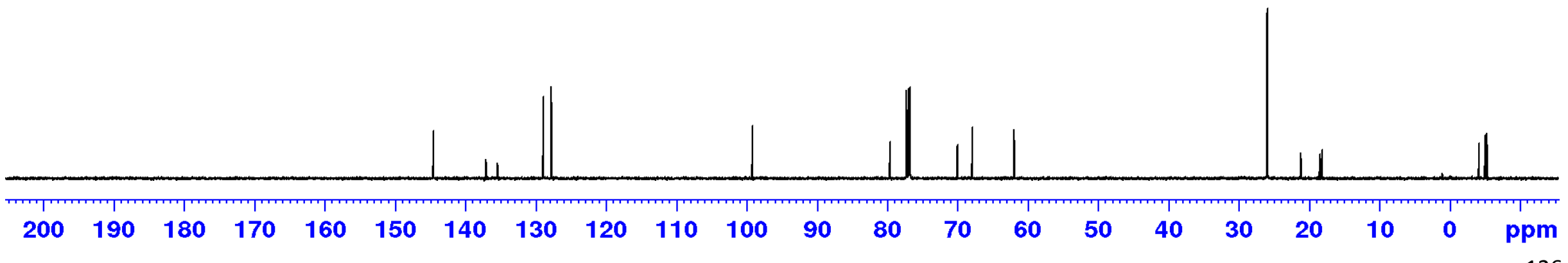




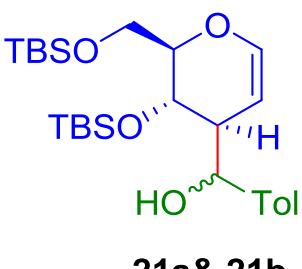

${ }^{1} \mathrm{H}$ NMR (500 MHz, $\mathrm{CDCl}_{3}$ )

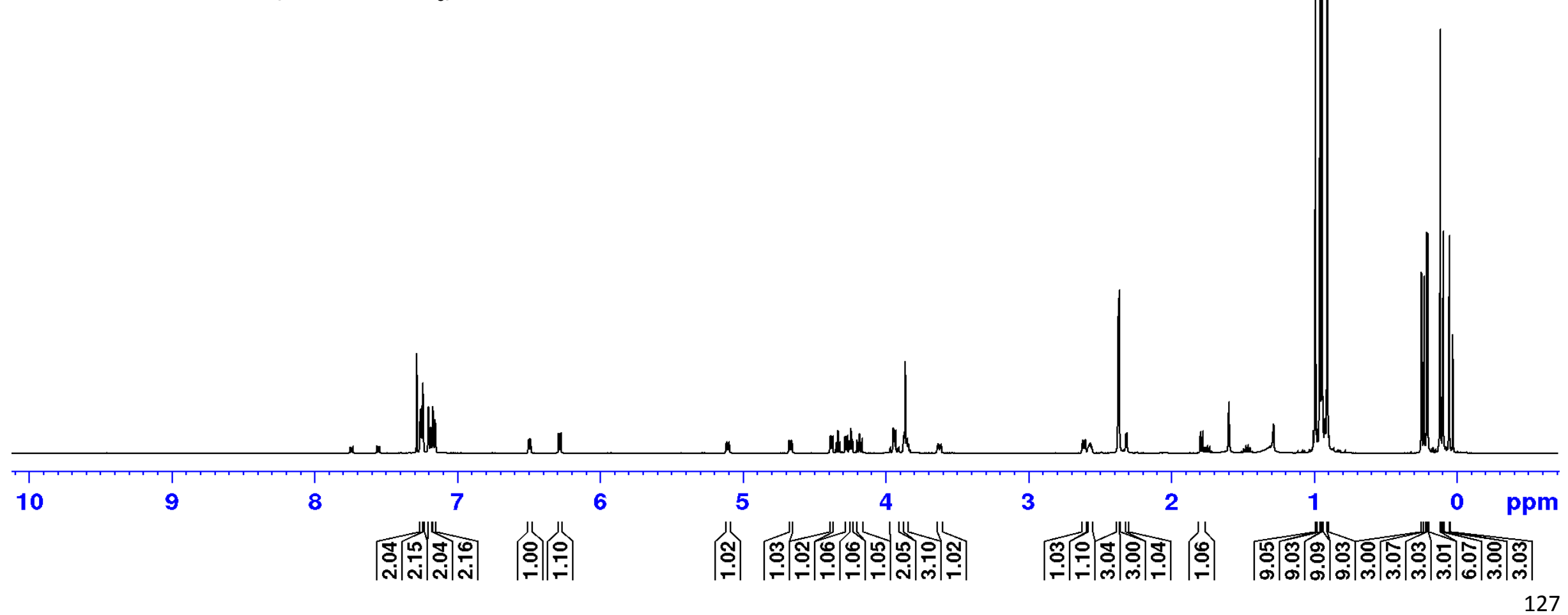




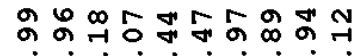
ปु่

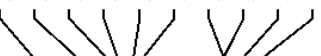

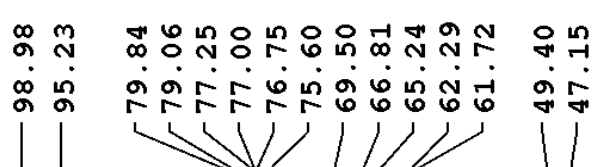

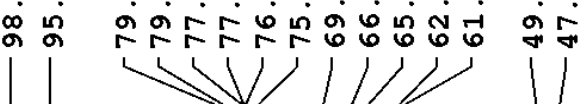

舟 ง

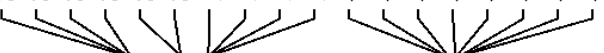

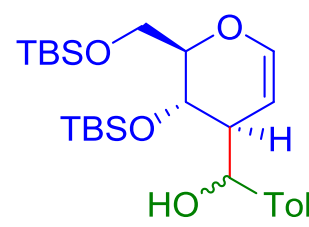

21a\& 21b

${ }^{13} \mathrm{C}$ NMR (125 MHz, $\mathrm{CDCl}_{3}$ )

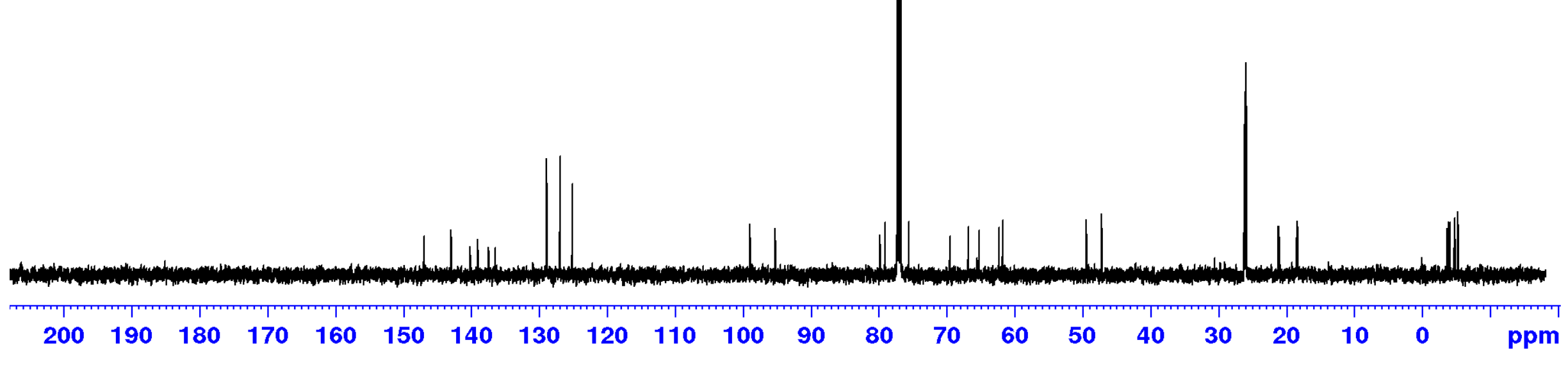




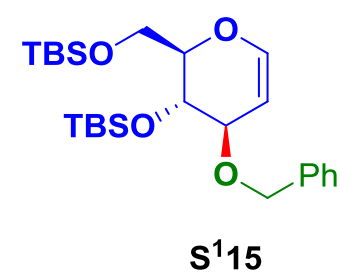

${ }^{1} \mathrm{H}$ NMR (500 MHz, $\mathrm{CDCl}_{3}$ )

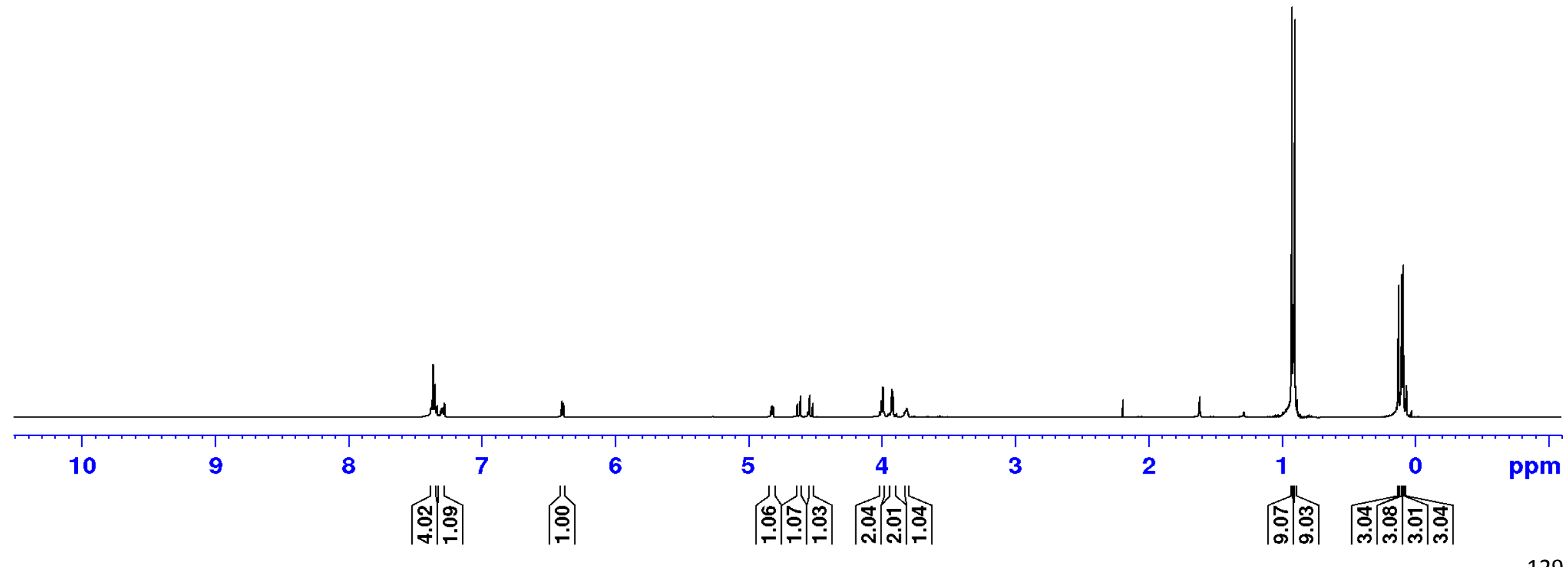




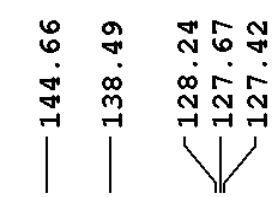

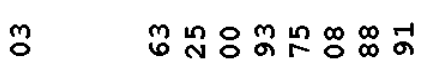

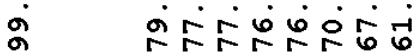

WVI

ㅇํㅇํํ육

มู่

$V^{N}$

ํํㅇㄱㅗ 우

विं एिं

iv

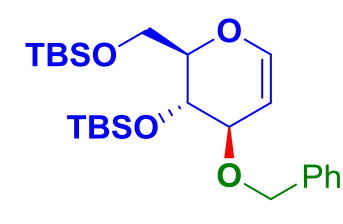

$S^{1} 15$

${ }^{13} \mathrm{C}$ NMR (125 MHz, $\mathrm{CDCl}_{3}$ )

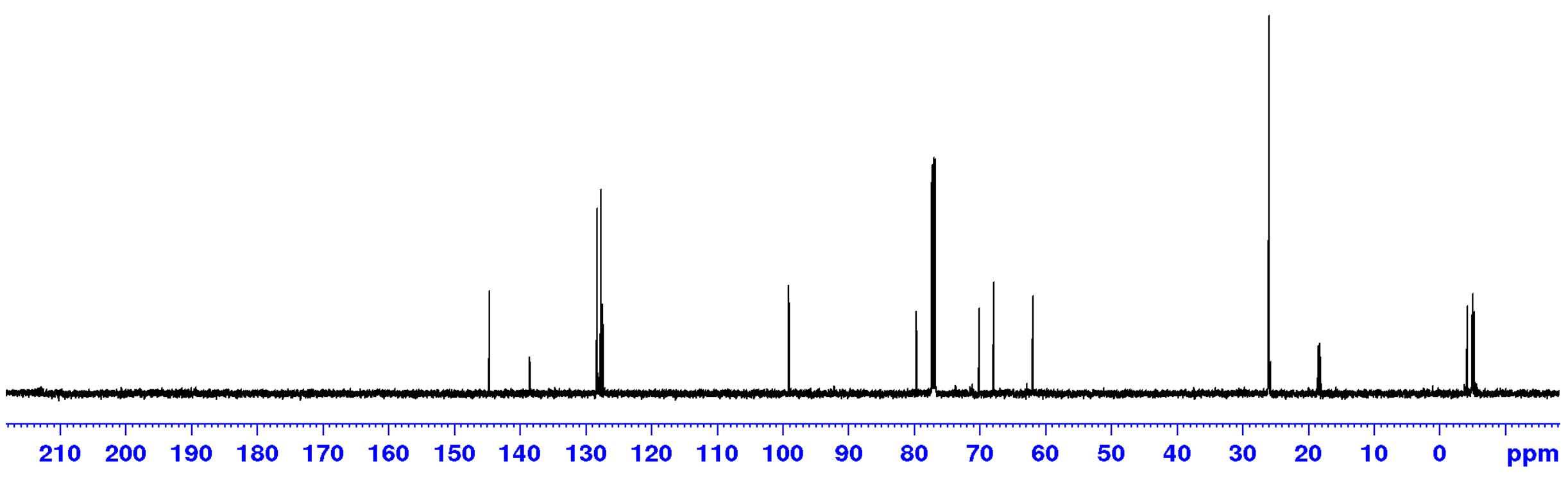




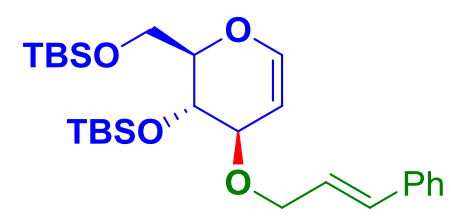

S22

${ }^{1} \mathrm{H}$ NMR (500 MHz, $\mathrm{CDCl}_{3}$ )

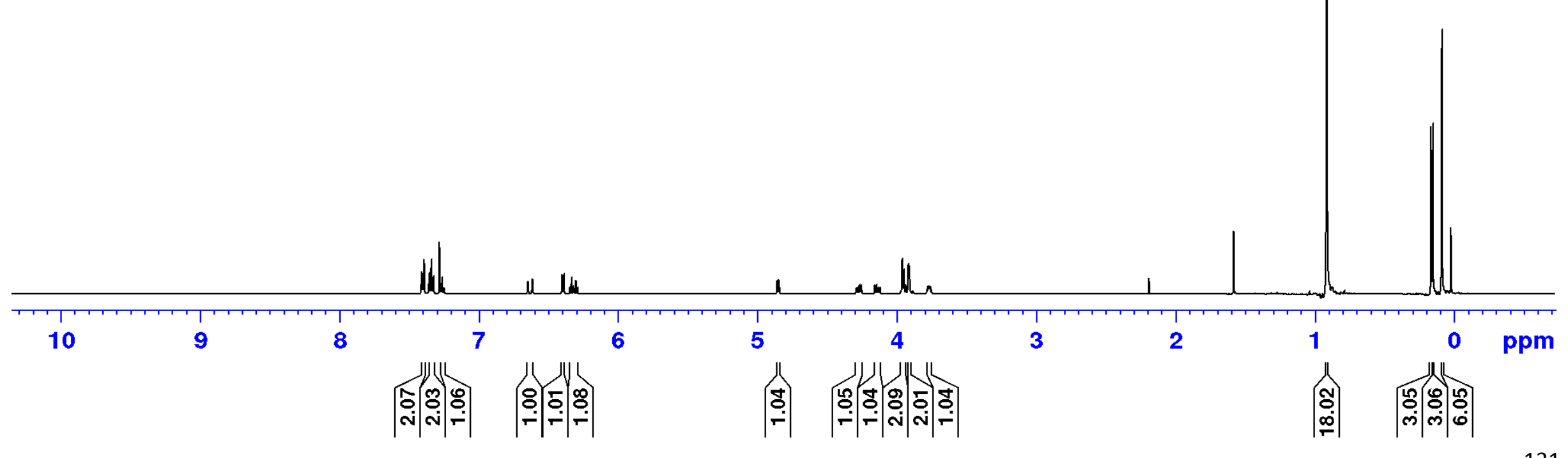



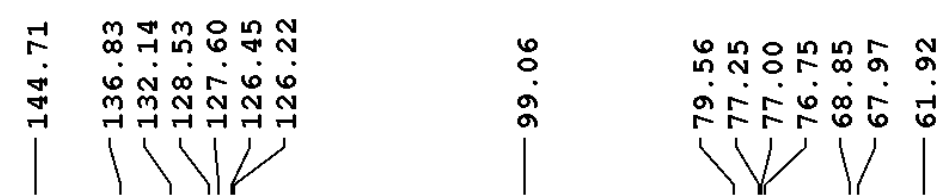

ผ

퐁응으

विं एं

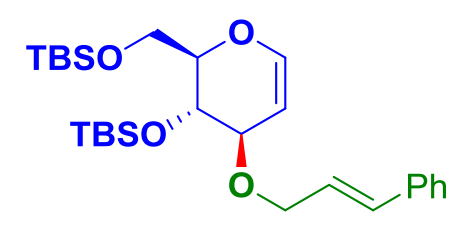

S22

${ }^{13} \mathrm{C}$ NMR (125 MHz, $\mathrm{CDCl}_{3}$ )

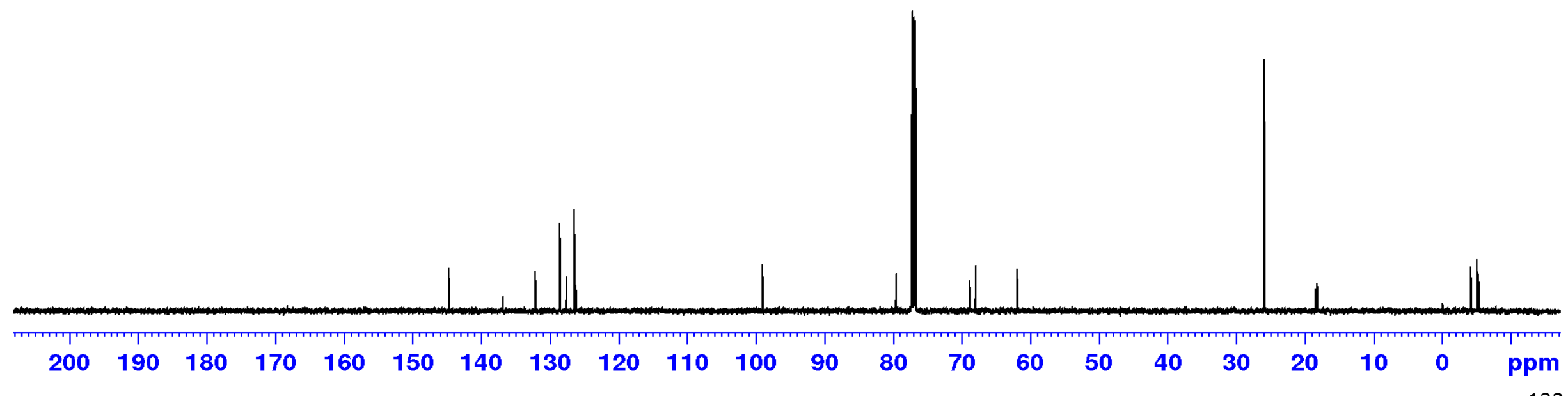




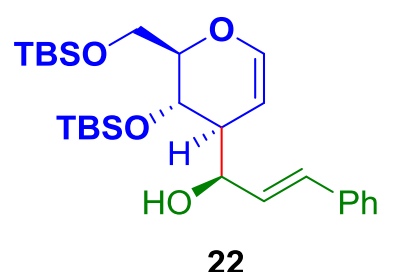

${ }^{1} \mathrm{H}$ NMR (500 MHz, $\mathrm{CDCl}_{3}$ )

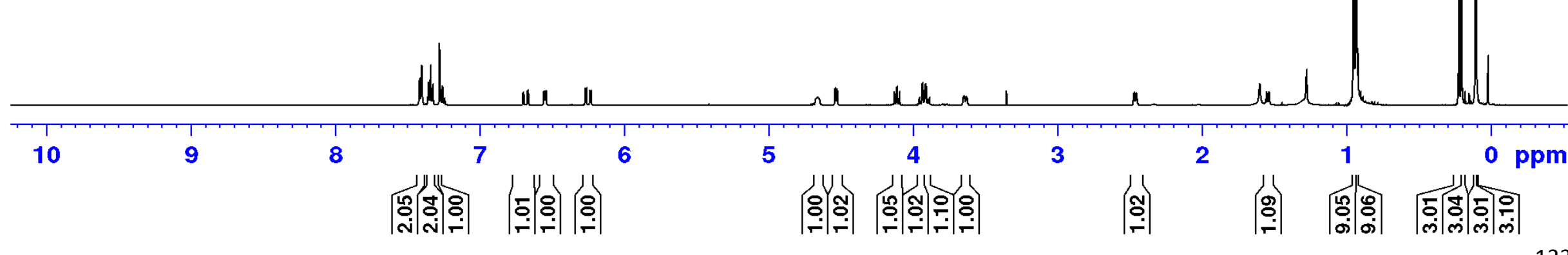




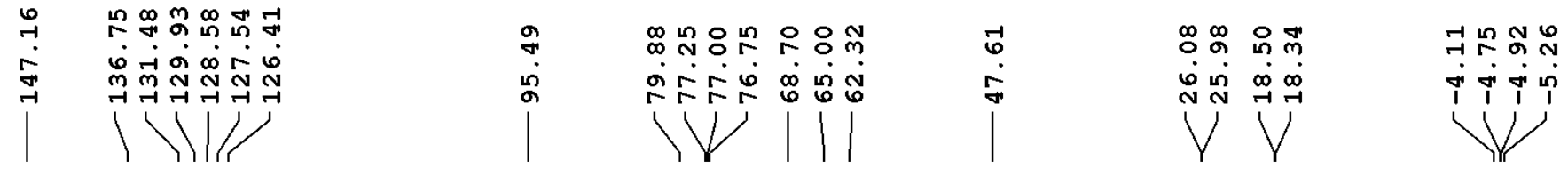

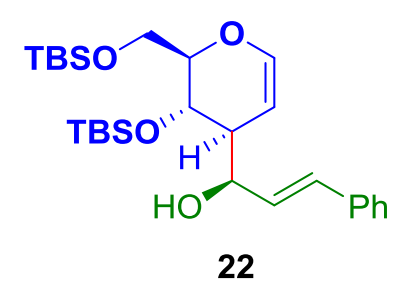

${ }^{13} \mathrm{C}$ NMR (125 MHz, $\mathrm{CDCl}_{3}$ )

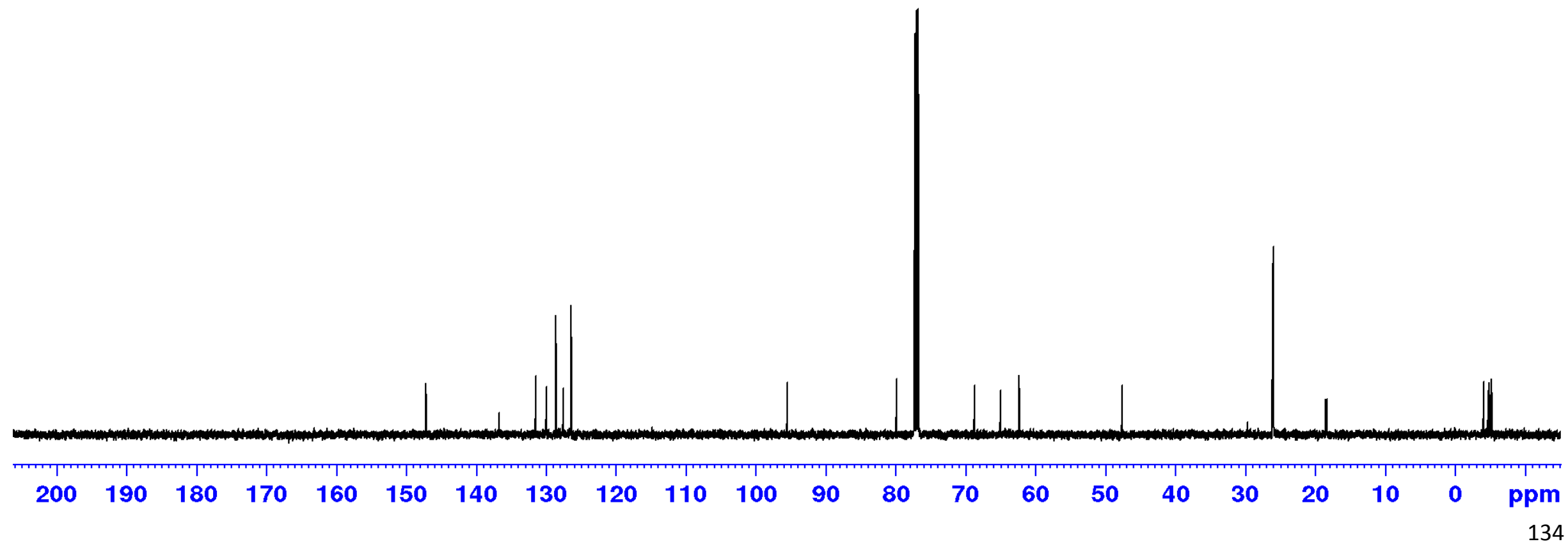




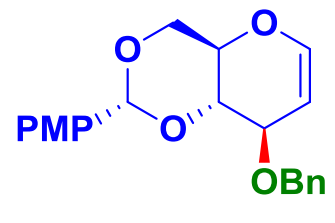

S23

${ }^{1} \mathrm{H}$ NMR (500 MHz, $\mathrm{CDCl}_{3}$ )

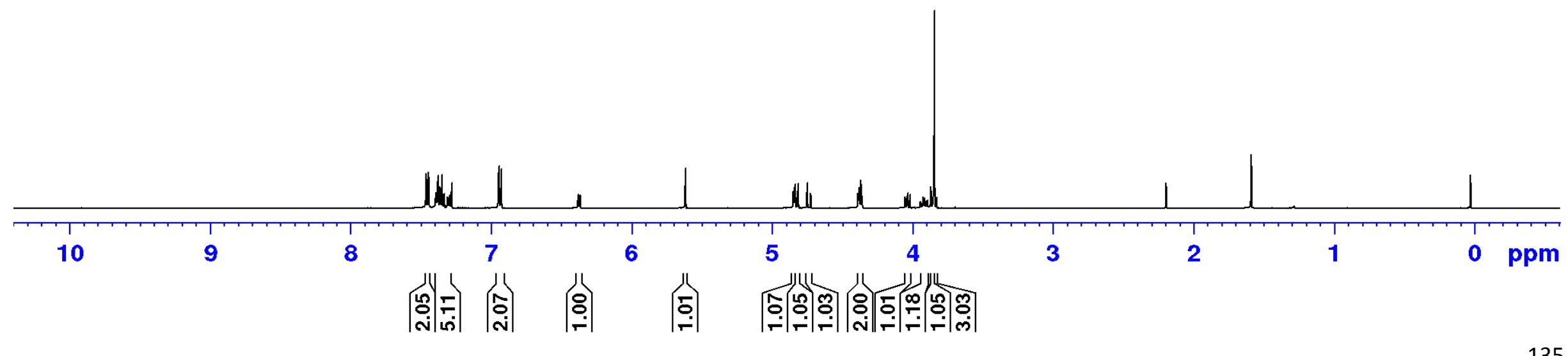




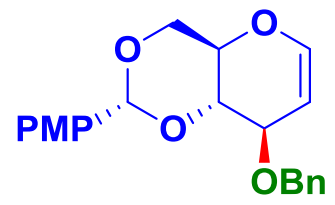

523

${ }^{13} \mathrm{C} \mathrm{NMR} \mathrm{(125} \mathrm{MHz,} \mathrm{CDCl}_{3}$ )

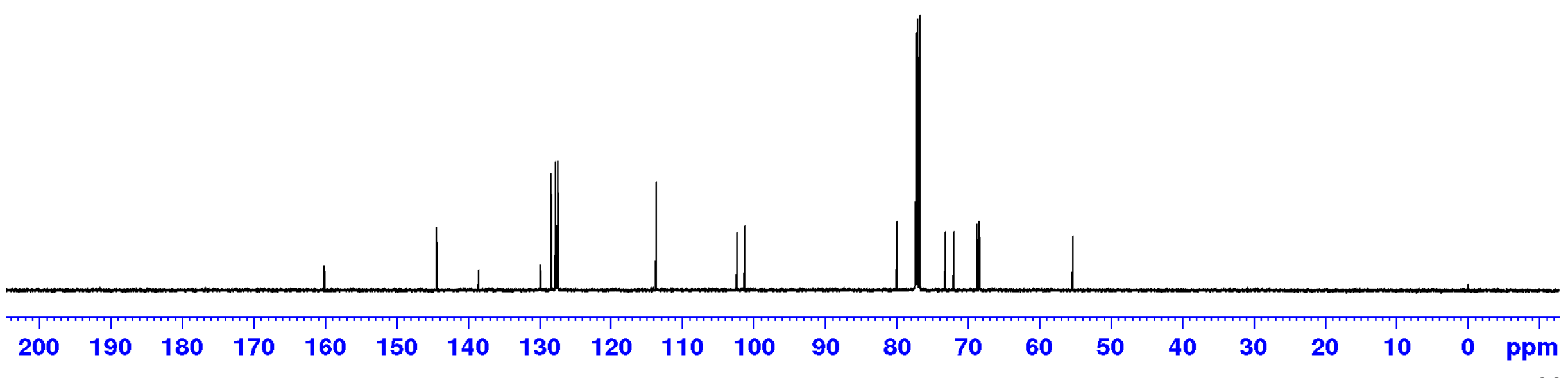




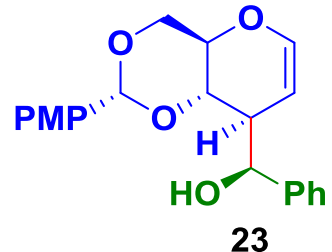

23

${ }^{1} \mathrm{H}$ NMR (500 MHz, $\mathrm{CDCl}_{3}$ )

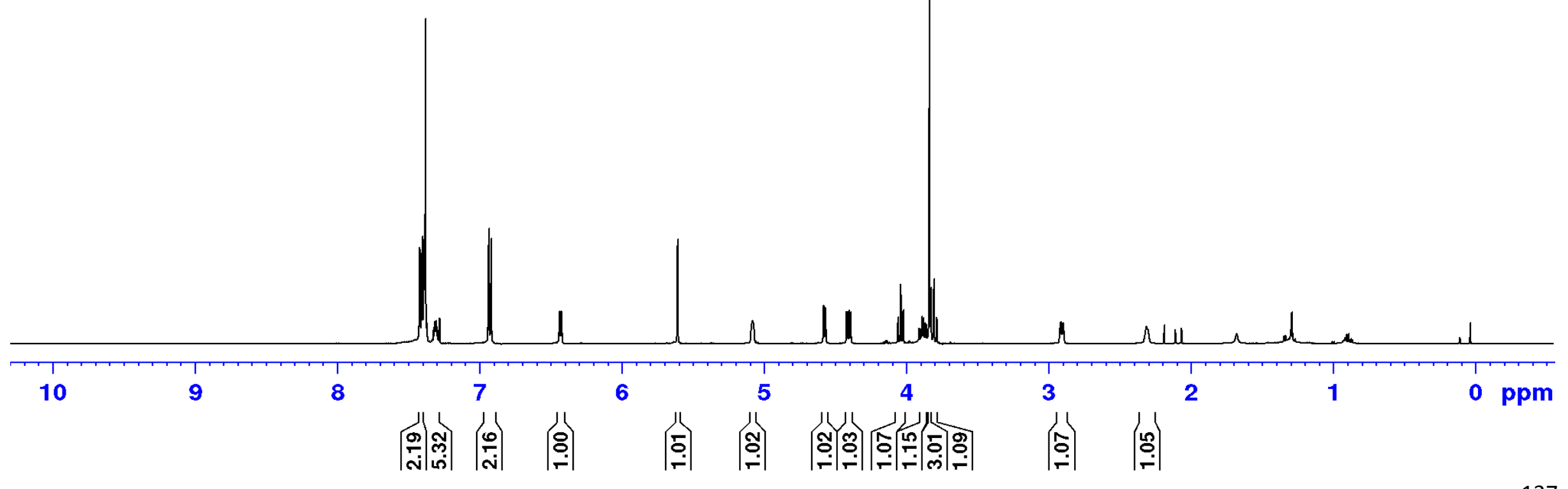

137 
| \&

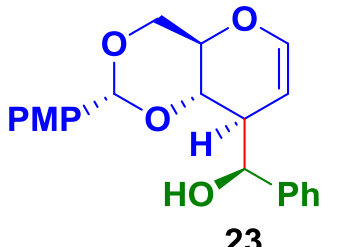

${ }^{13} \mathrm{C}$ NMR (125 MHz, $\mathrm{CDCl}_{3}$ )

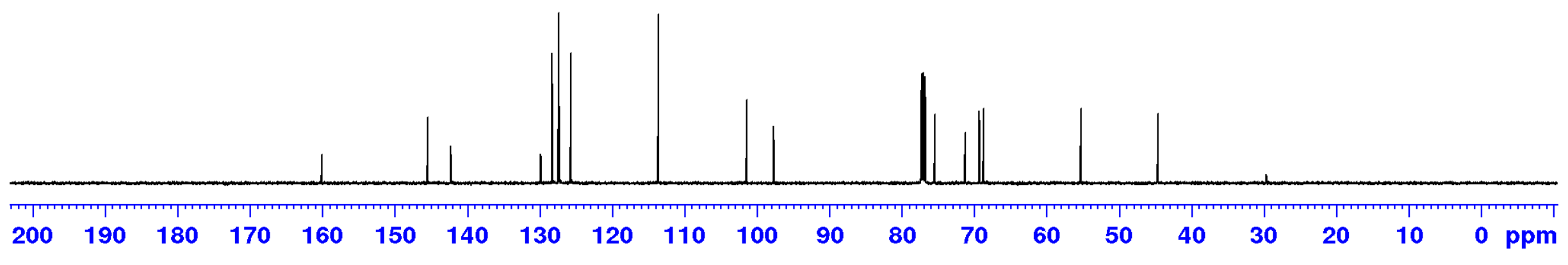




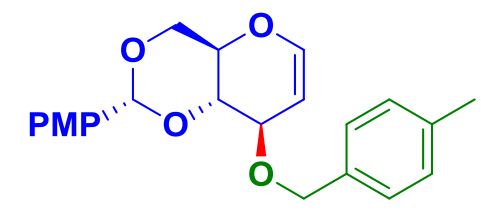

S24

${ }^{1} \mathrm{H}$ NMR (500 MHz, $\mathrm{CDCl}_{3}$ )

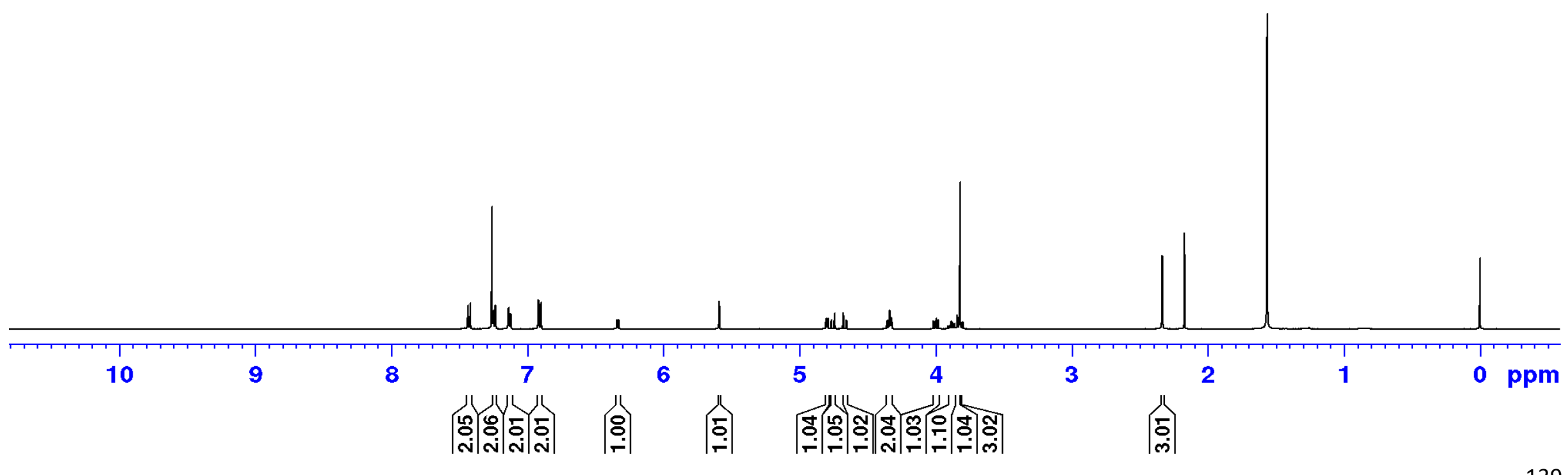




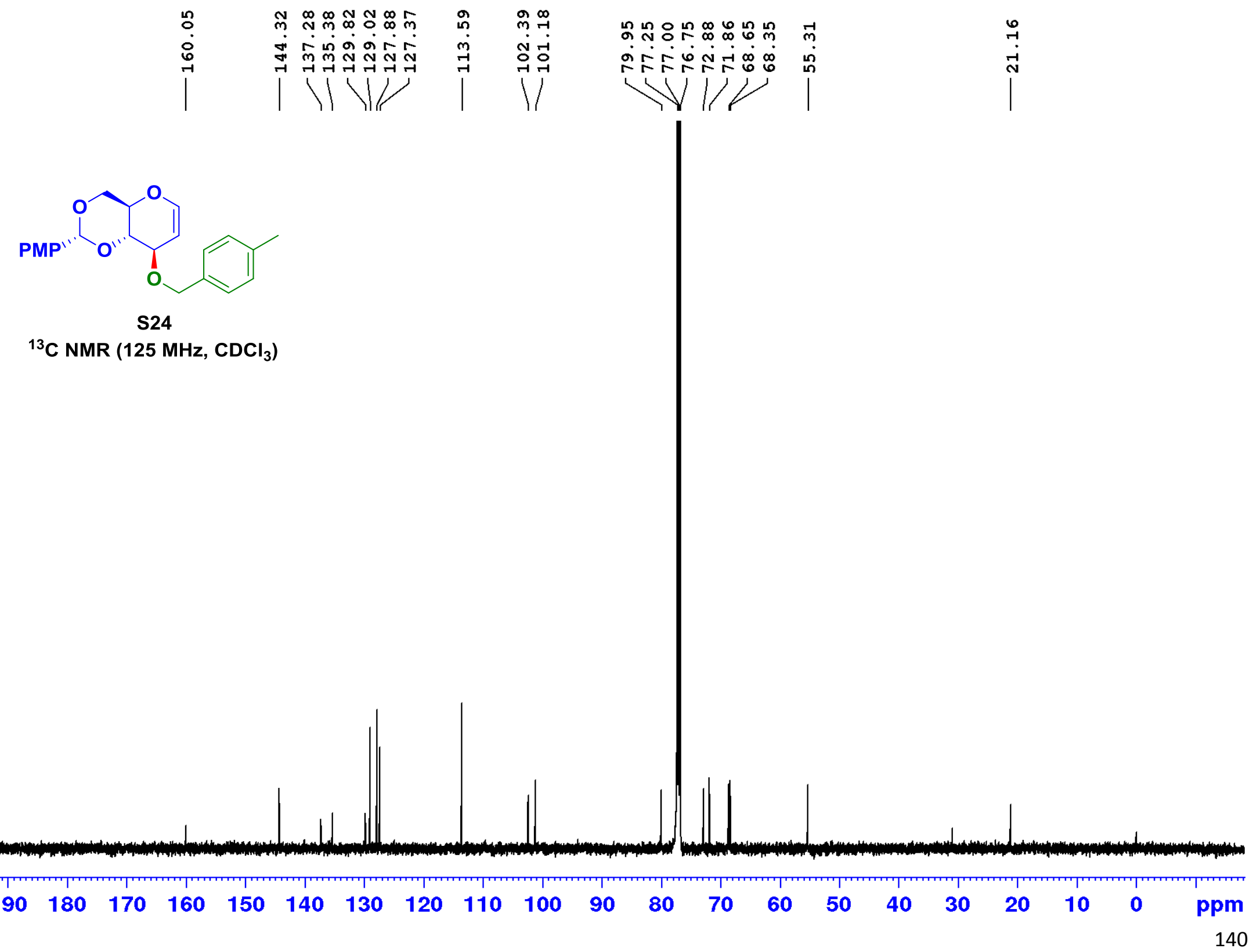




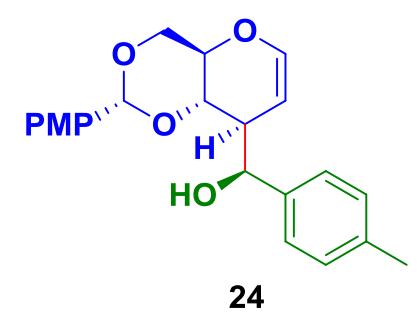

${ }^{1} \mathrm{H}$ NMR (400 MHz, $\mathrm{CDCl}_{3}$ )

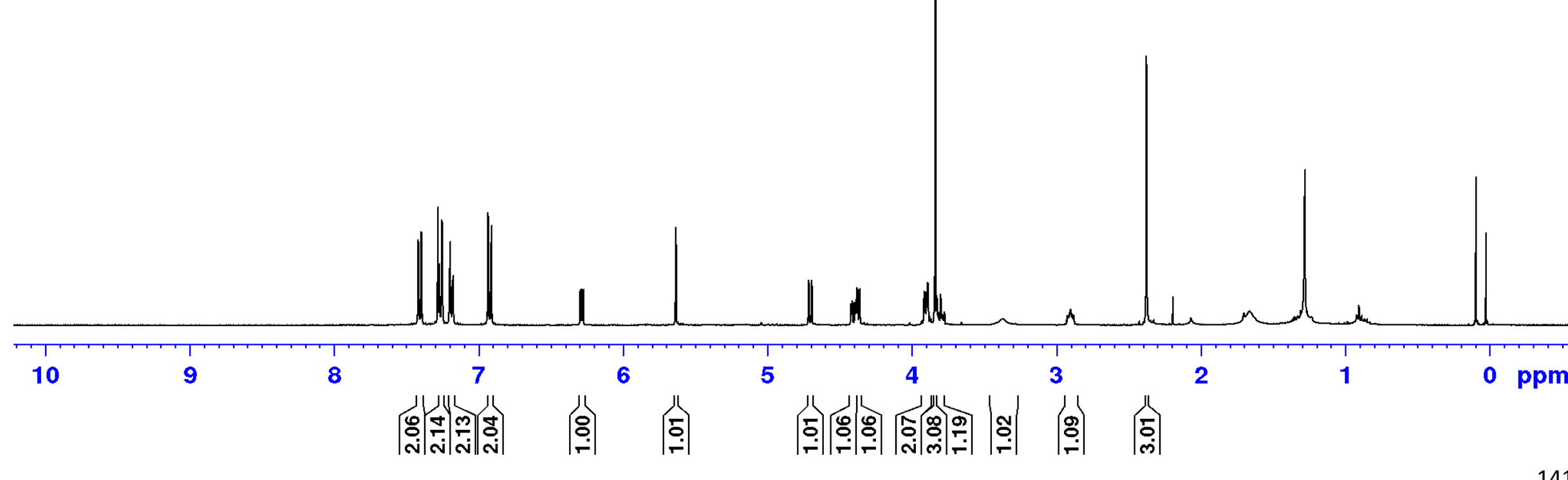




|

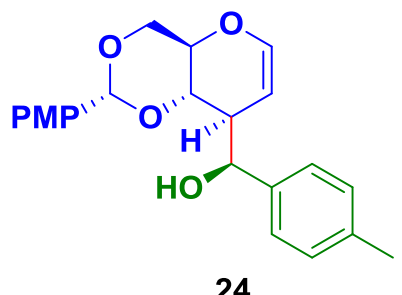

$\left.{ }^{13} \mathrm{C} \mathrm{NMR} \mathrm{(100} \mathrm{MHz,} \mathrm{CDCl}_{3}\right)$

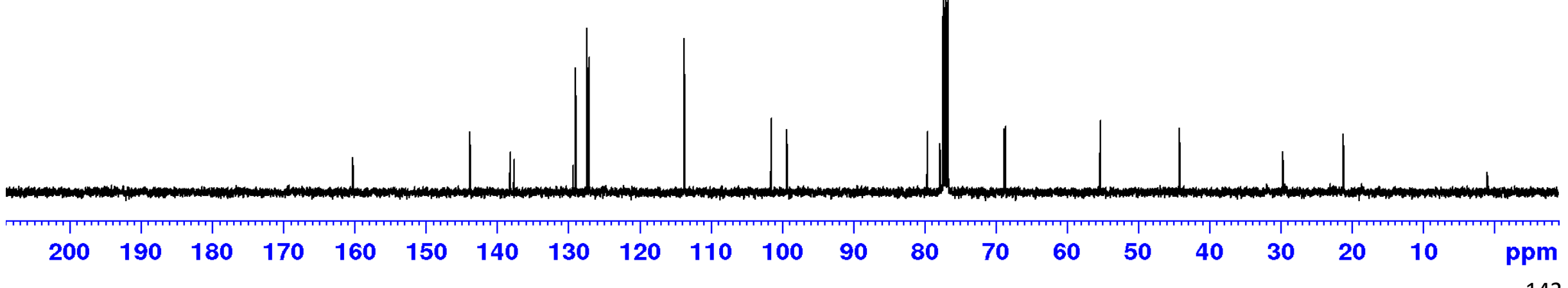




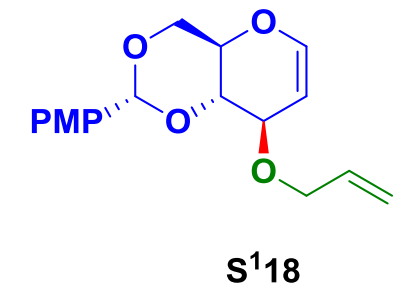

${ }^{1} \mathrm{H}$ NMR (500 MHz, $\mathrm{CDCl}_{3}$ )

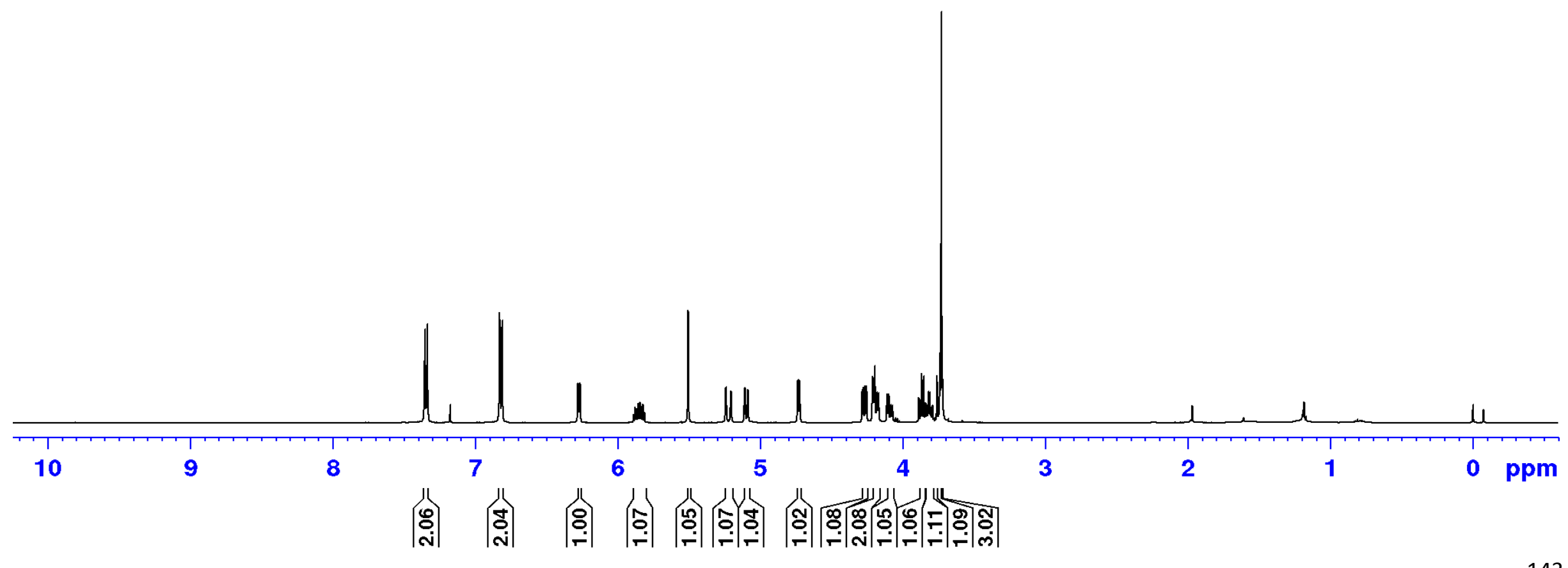




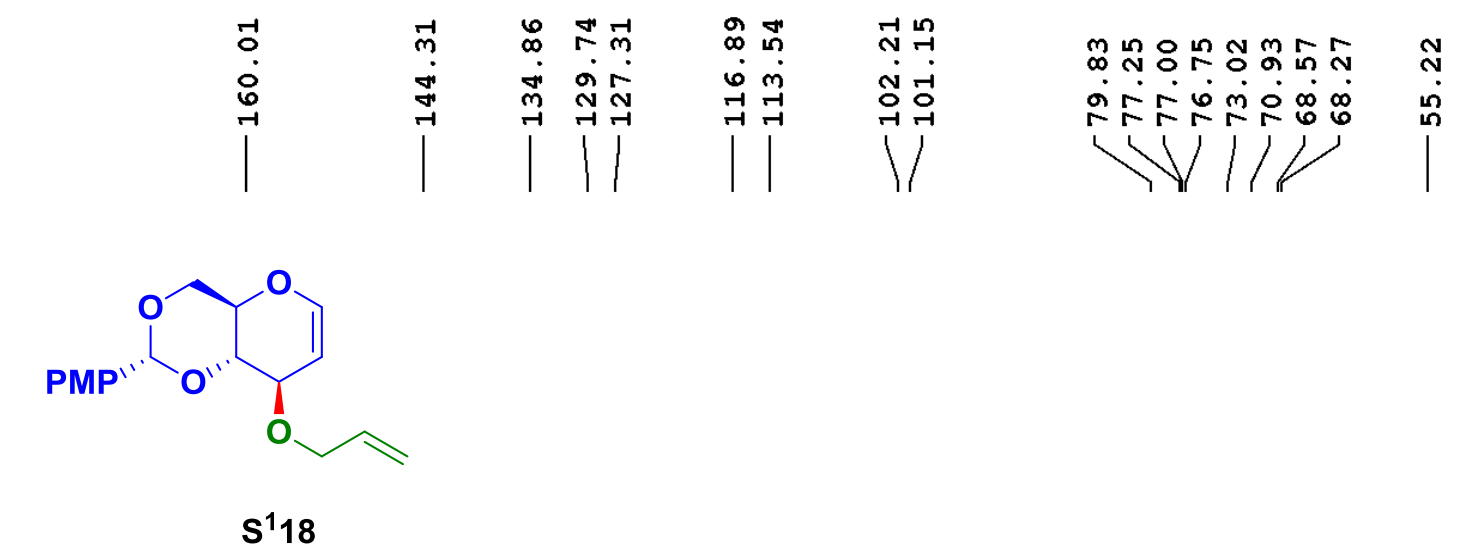

${ }^{13} \mathrm{C}$ NMR (125 MHz, $\mathrm{CDCl}_{3}$ )

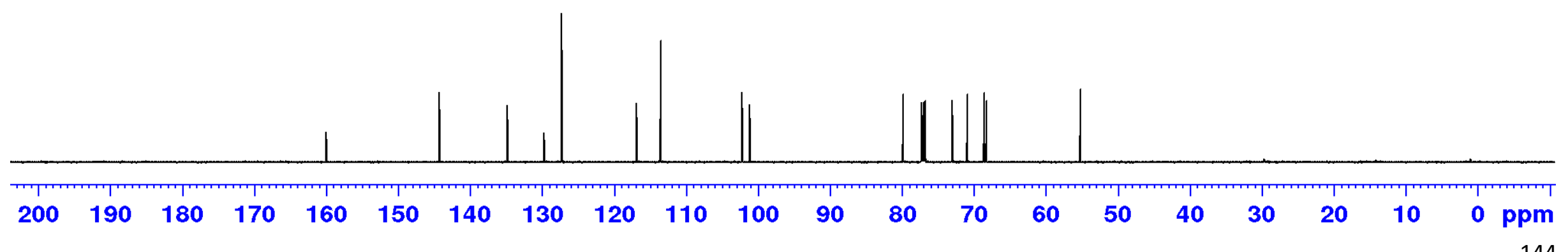




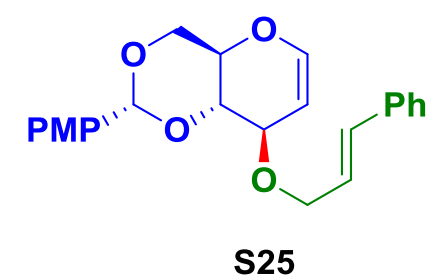

${ }^{1} \mathrm{H}$ NMR (500 MHz, $\mathrm{CDCl}_{3}$ )

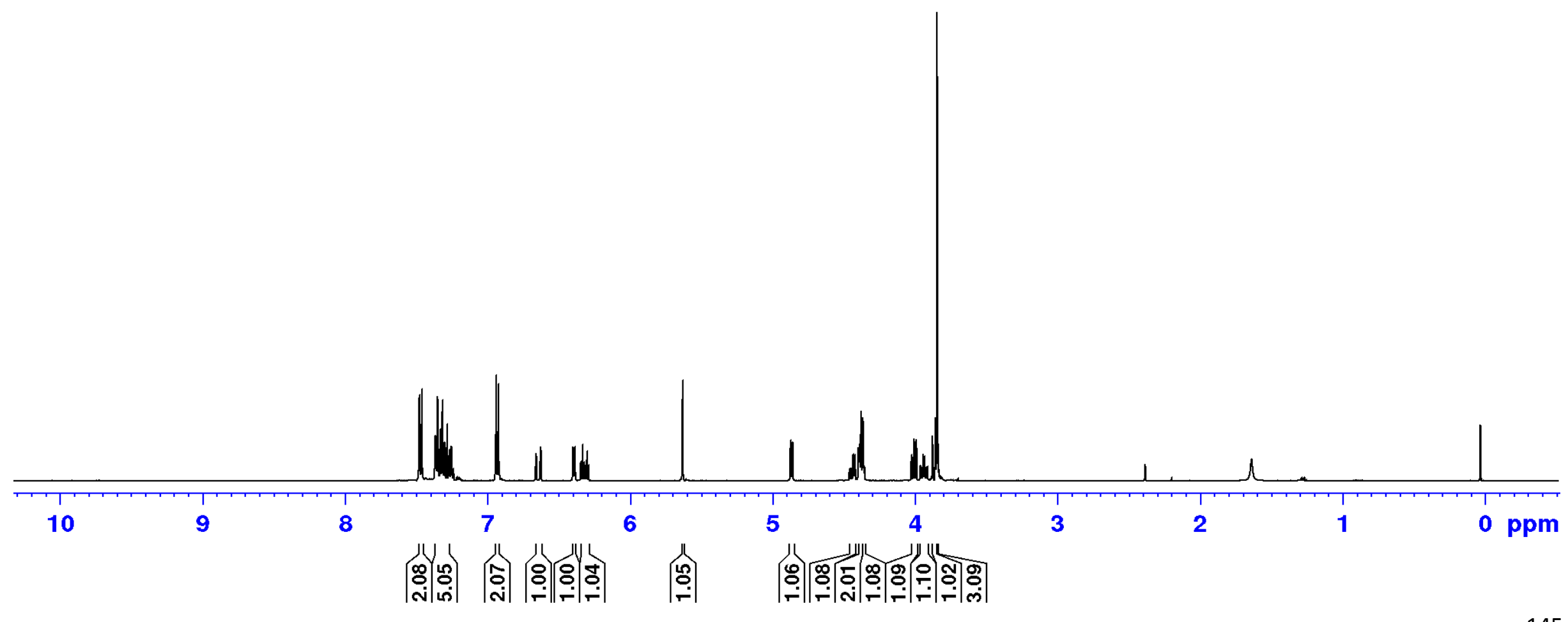



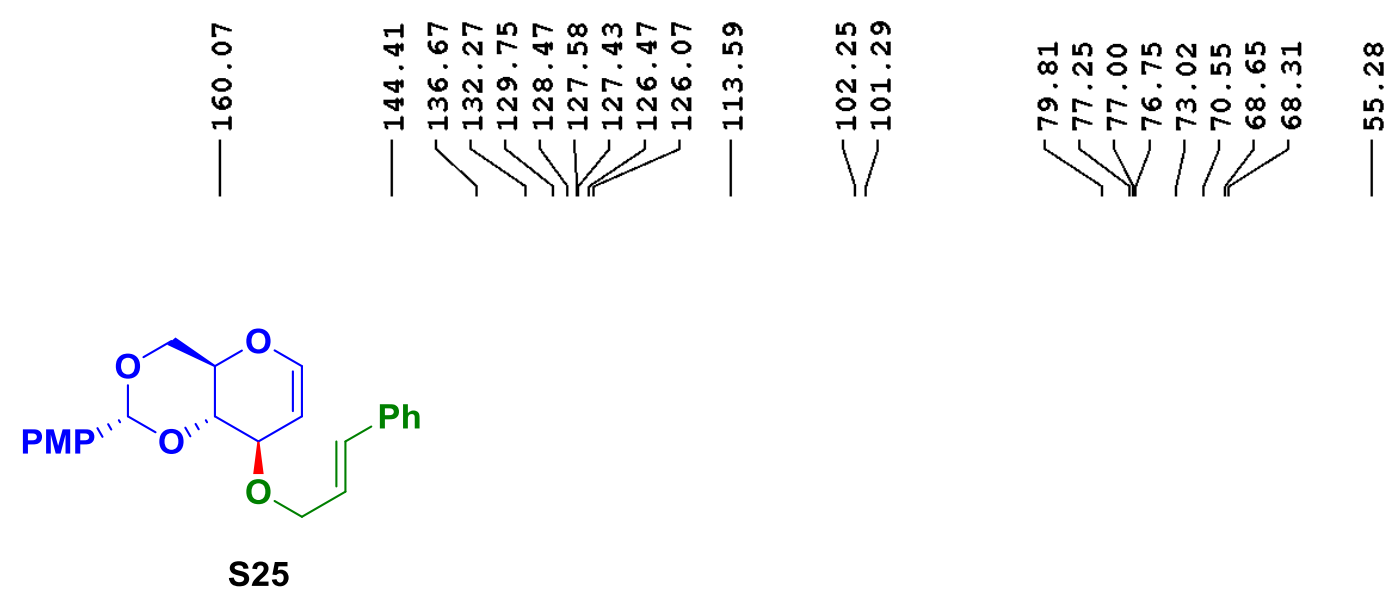

${ }^{13} \mathrm{C}$ NMR (125 MHz, $\mathrm{CDCl}_{3}$ )

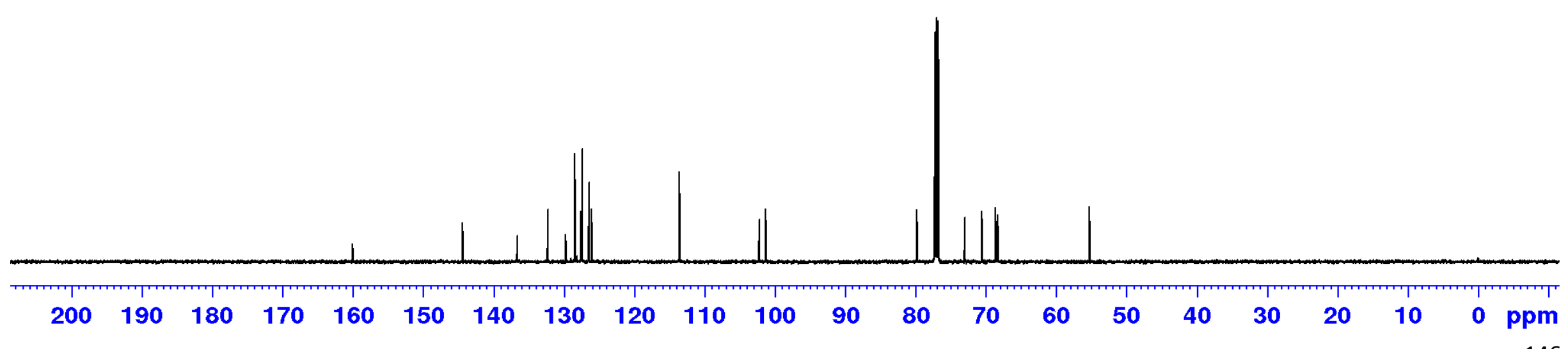




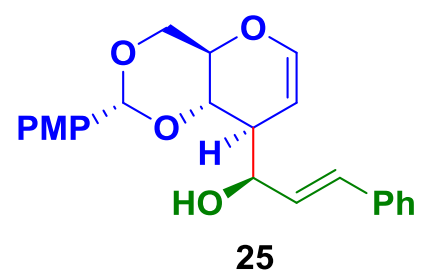

${ }^{1} \mathrm{H}$ NMR (400 MHz, $\mathrm{CDCl}_{3}$ )

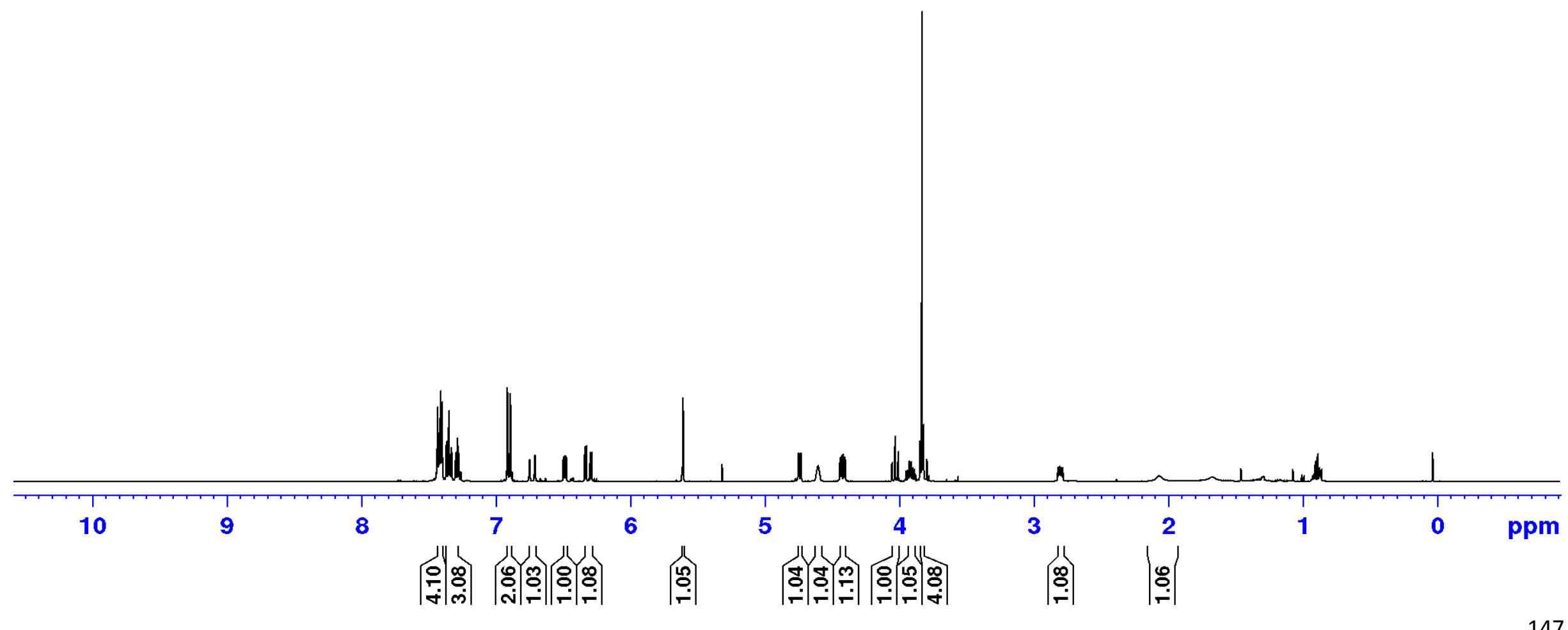



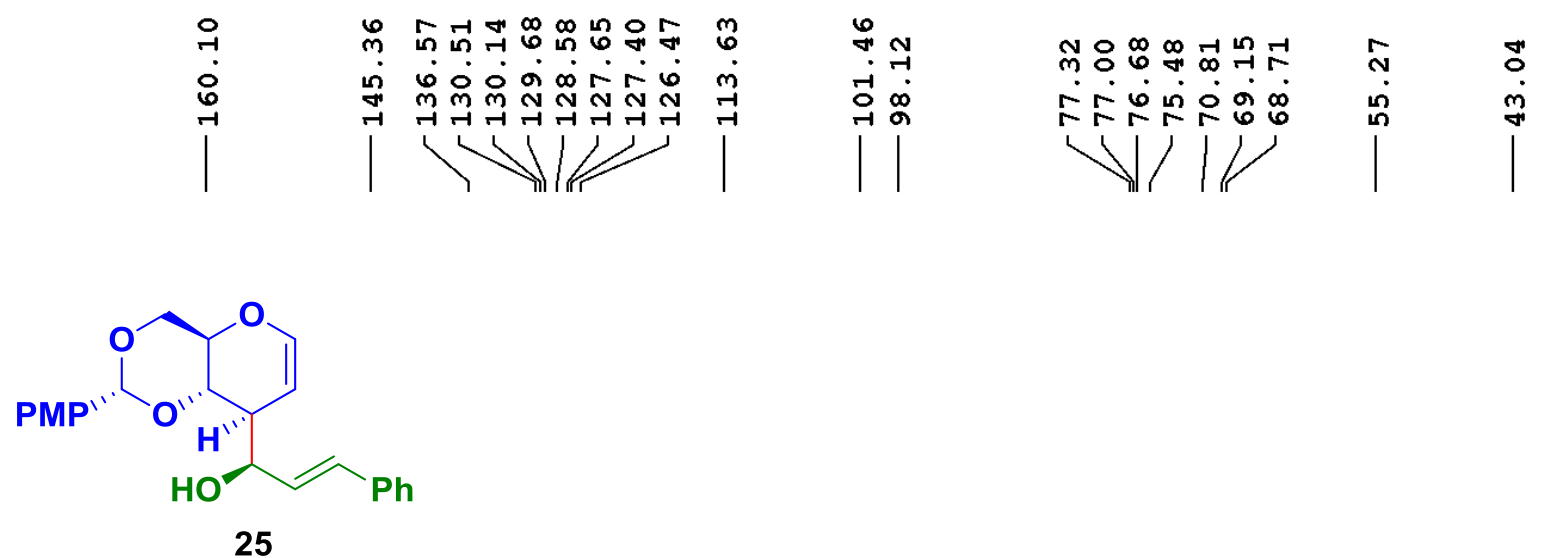

${ }^{13} \mathrm{C}$ NMR $\left(100 \mathrm{MHz}, \mathrm{CDCl}_{3}\right)$

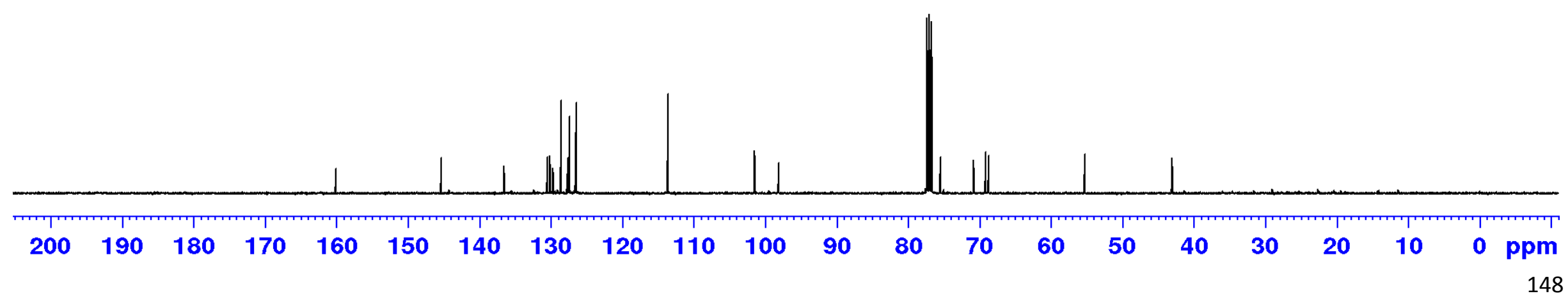




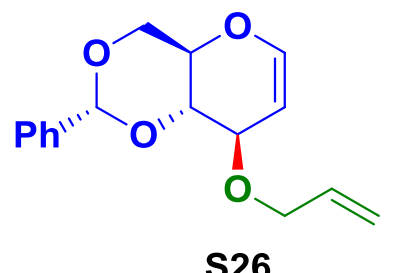

${ }^{1} \mathrm{H}$ NMR (500 MHz, $\mathrm{CDCl}_{3}$ )

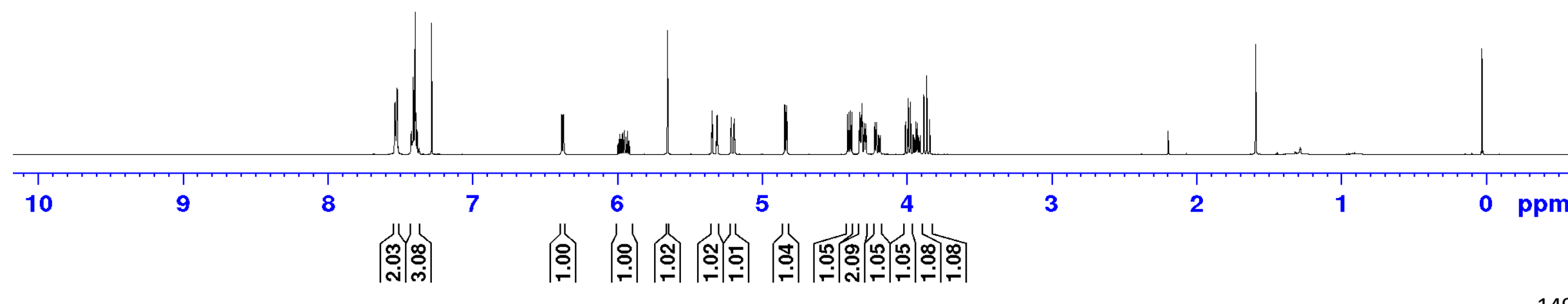



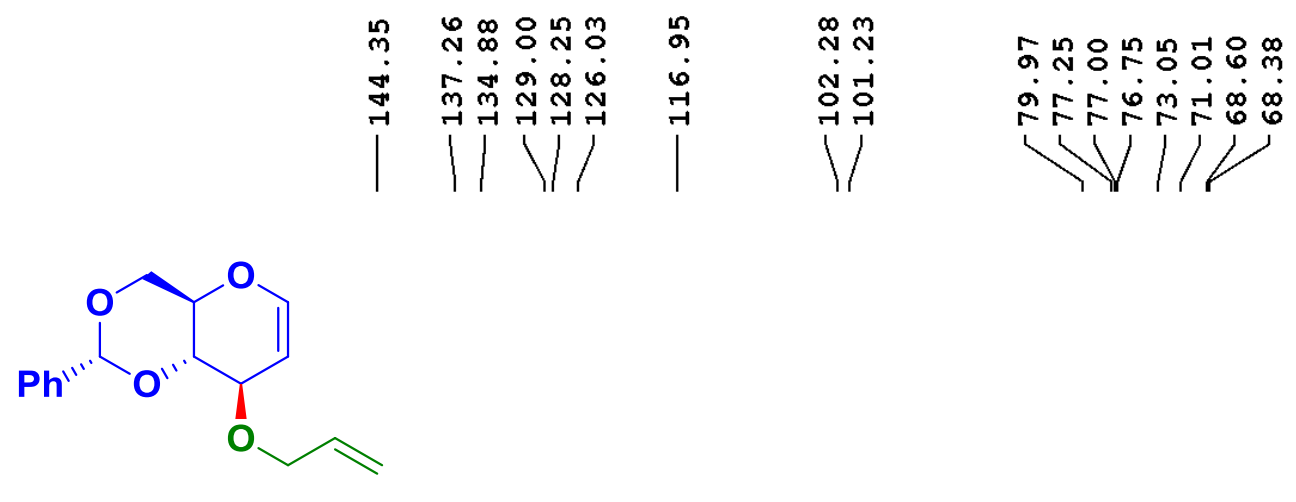

S26

${ }^{13} \mathrm{C}$ NMR (125 MHz, $\mathrm{CDCl}_{3}$ )

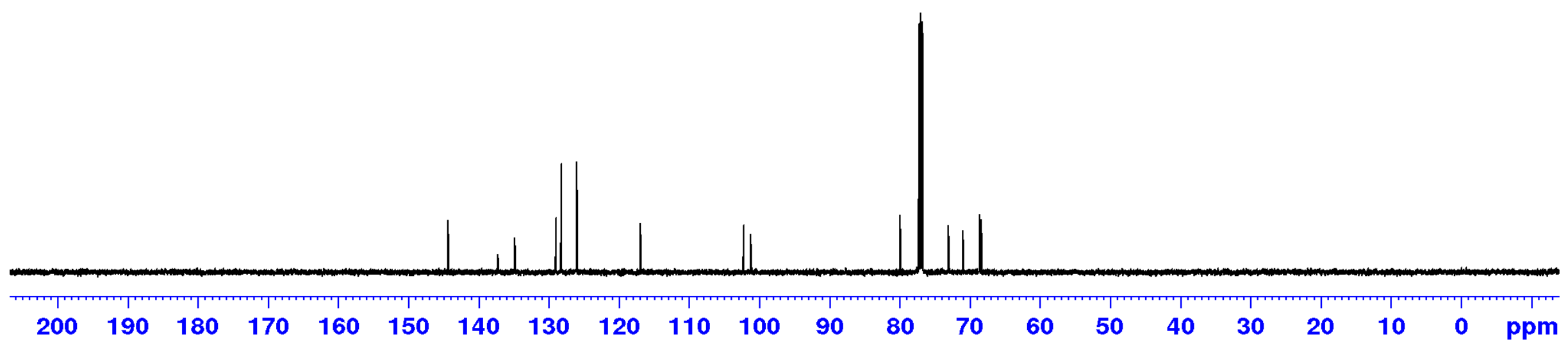




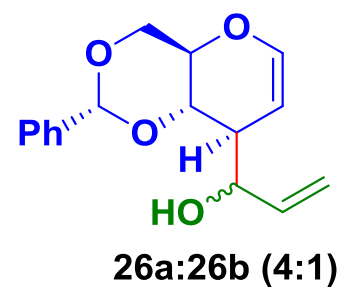

${ }^{1} \mathrm{H}$ NMR (500 MHz, $\mathrm{CDCl}_{3}$ )

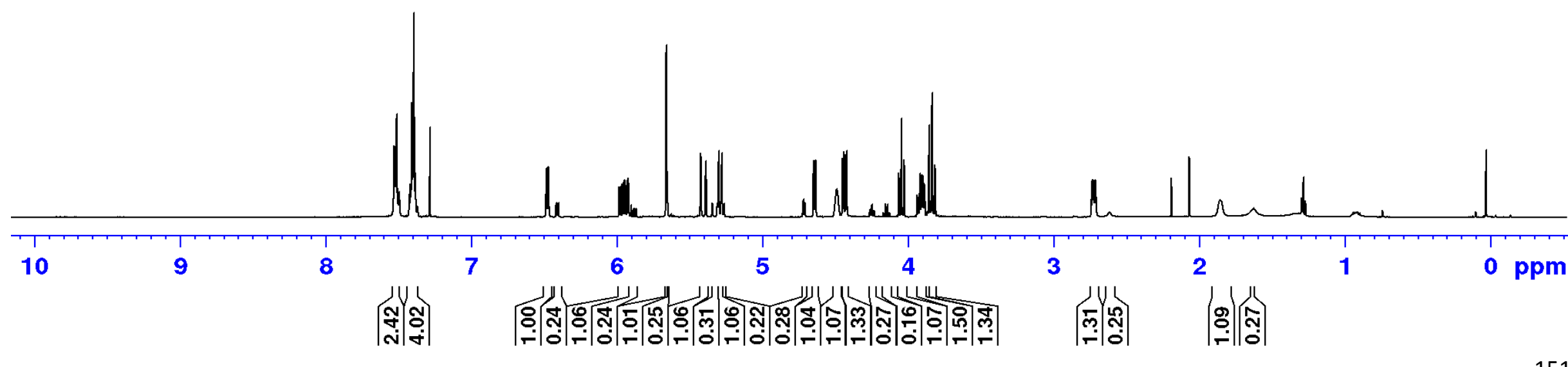




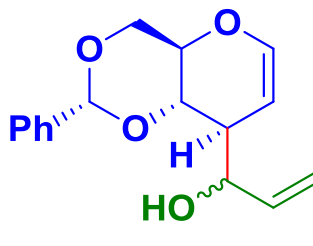

26a:26b (4:1)

${ }^{13} \mathrm{C}$ NMR (125 MHz, $\left.\mathrm{CDCl}_{3}\right)$

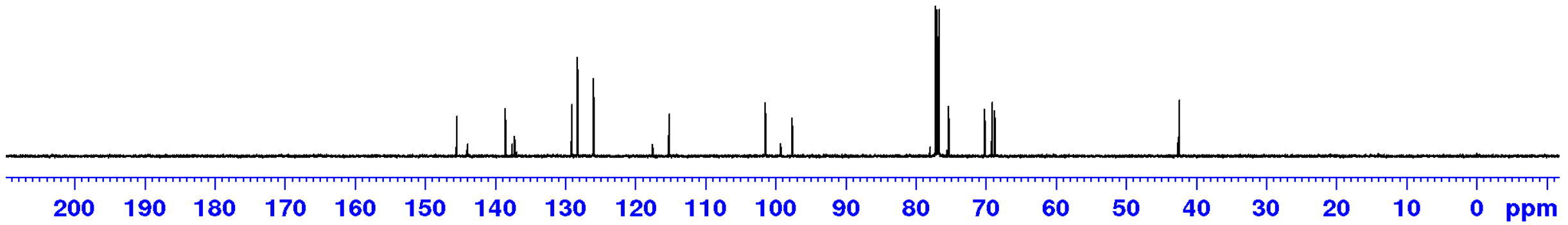




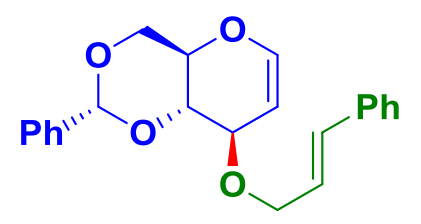

S27

${ }^{1} \mathrm{H}$ NMR (500 MHz, $\mathrm{CDCl}_{3}$ )

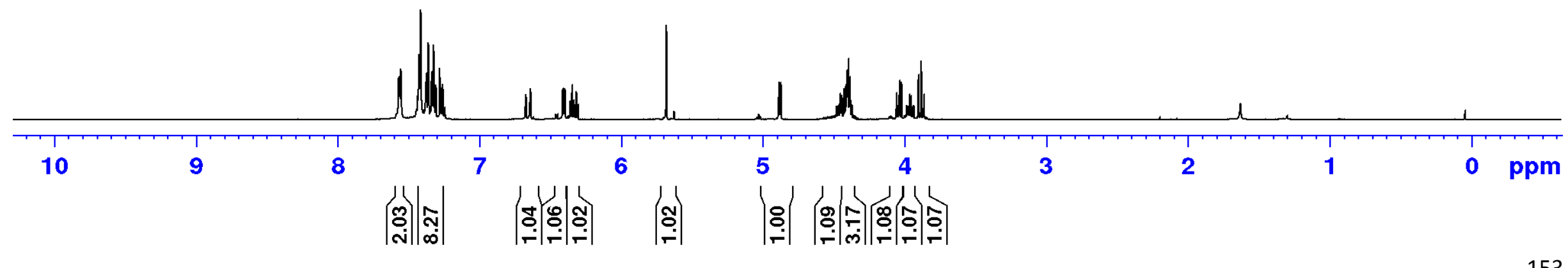



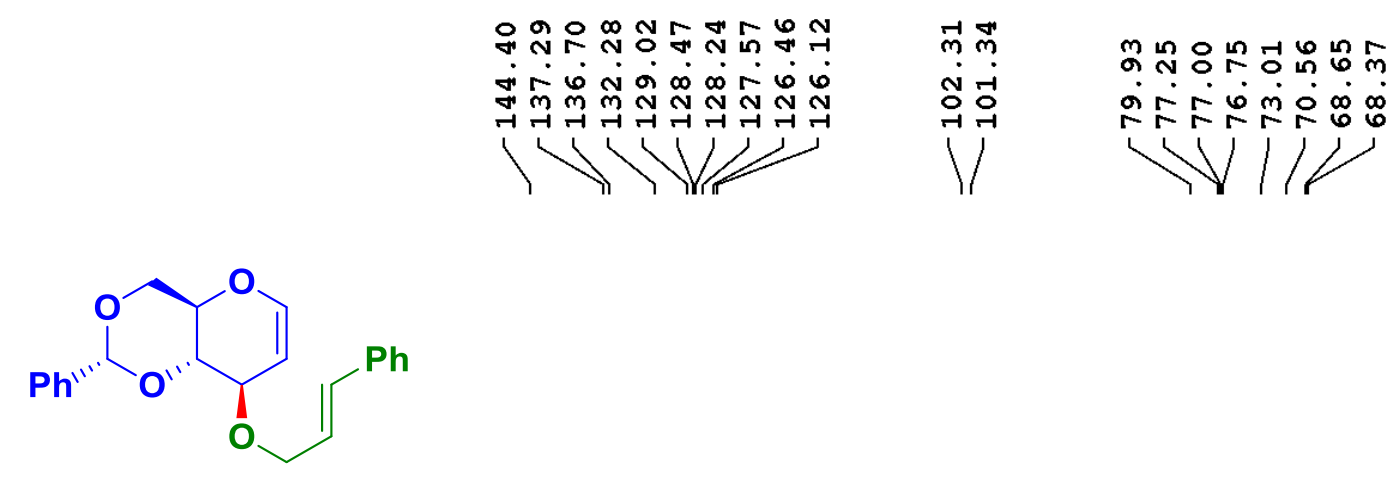

527

${ }^{13} \mathrm{C}$ NMR (125 MHz, $\mathrm{CDCl}_{3}$ )

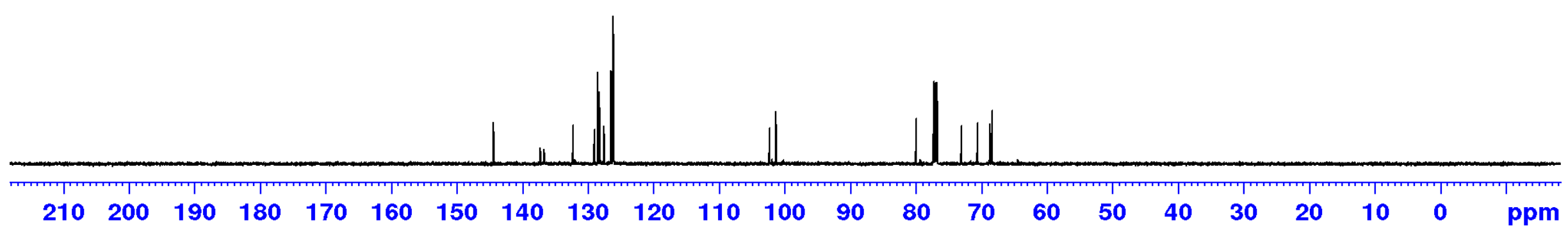




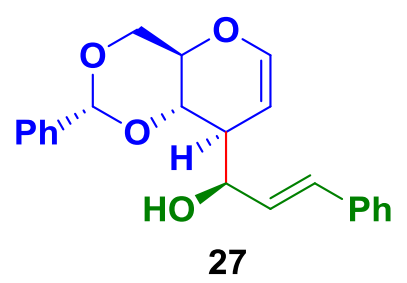

${ }^{1} \mathrm{H}$ NMR (500 MHz, $\left.\mathrm{CDCl}_{3}\right)$

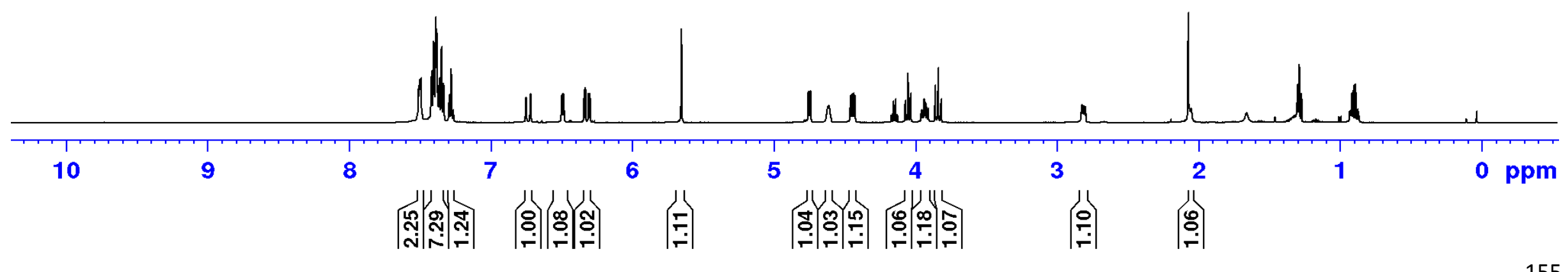




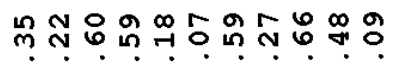
भु户்

>

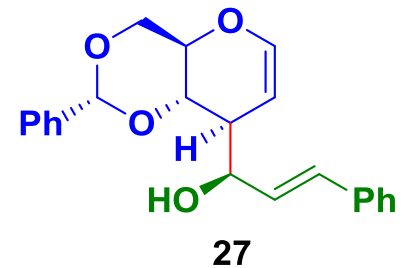

${ }^{13} \mathrm{C}$ NMR (125 MHz, $\mathrm{CDCl}_{3}$ )

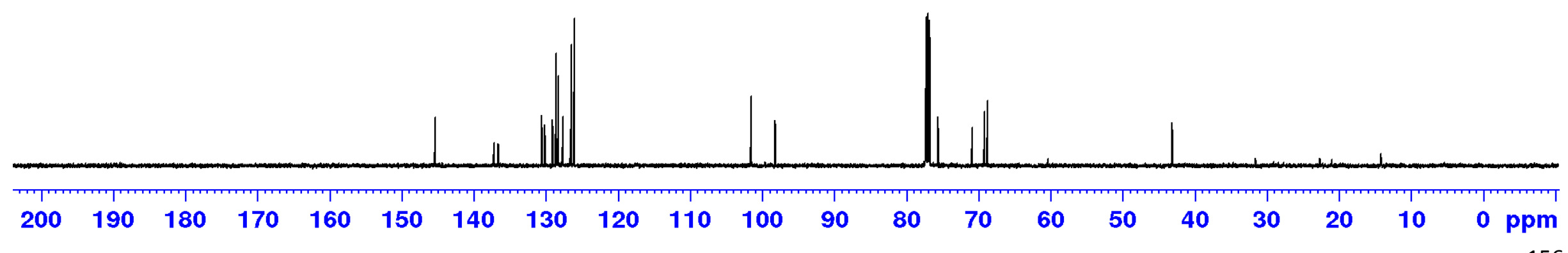




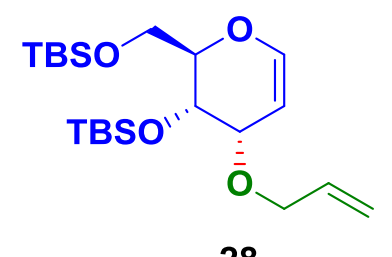

28

${ }^{1} \mathrm{H}$ NMR (500 $\mathrm{MHz}, \mathrm{CDCl}_{3}$ )

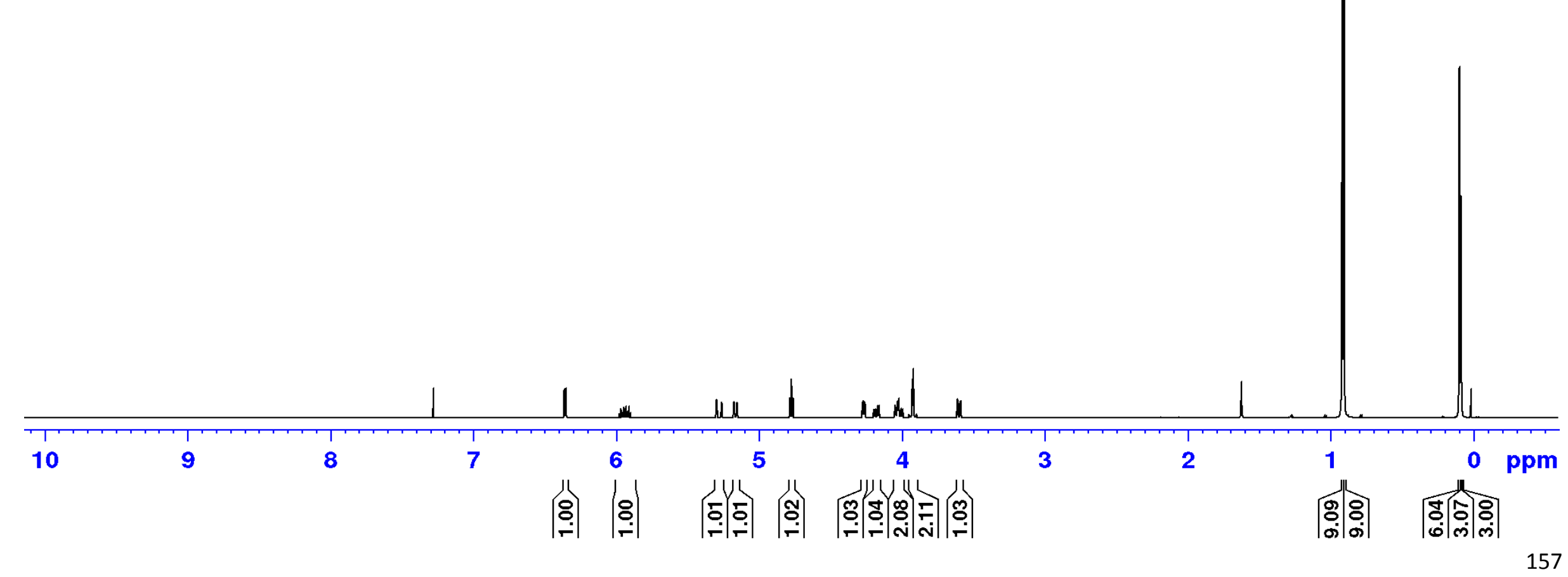




N

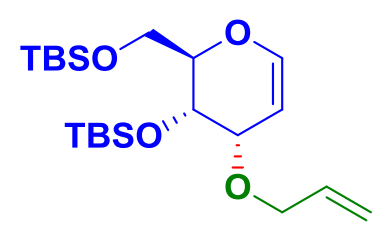

28

${ }^{13} \mathrm{C}$ NMR (125 MHz, $\mathrm{CDCl}_{3}$ )

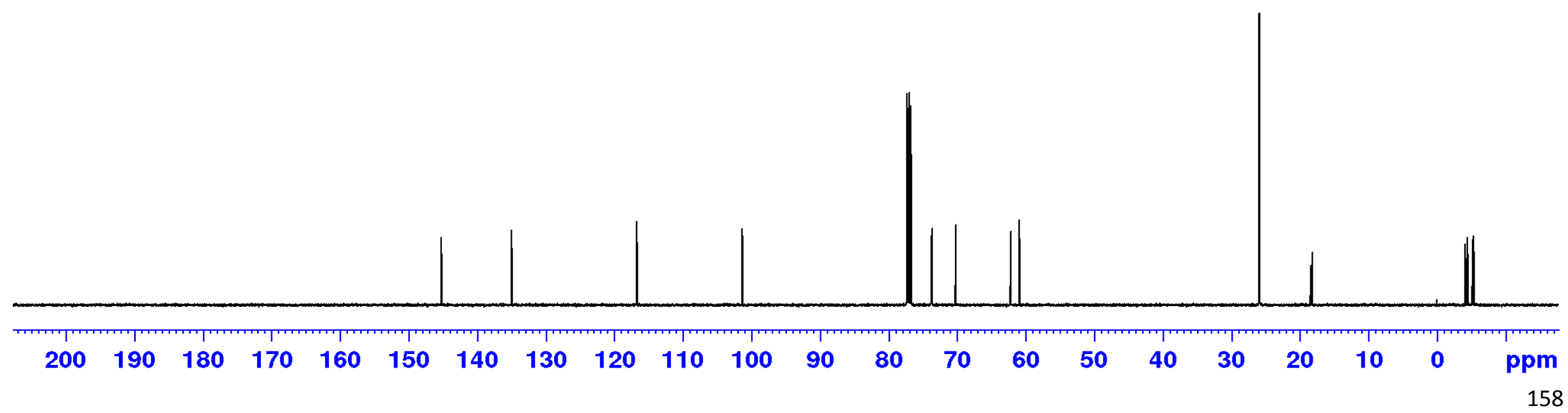




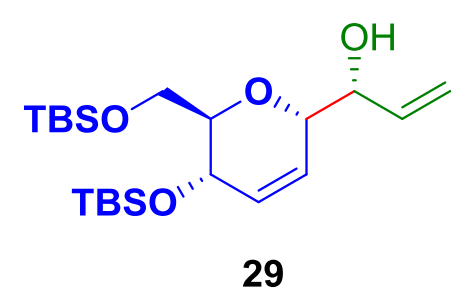

${ }^{1} \mathrm{H}$ NMR (500 MHz, $\mathrm{CDCl}_{3}$ )

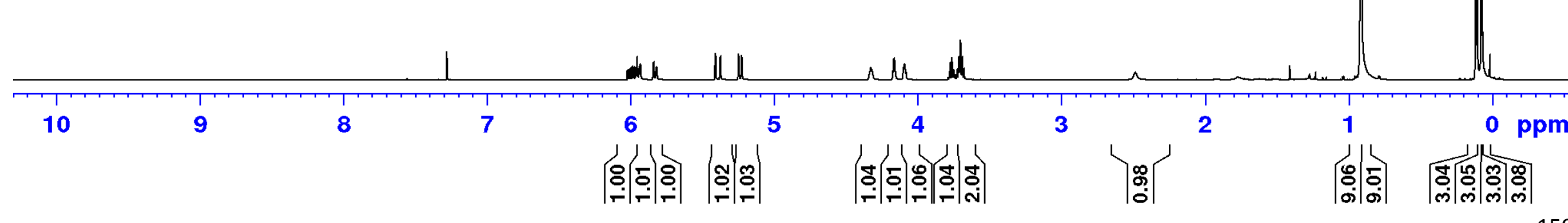




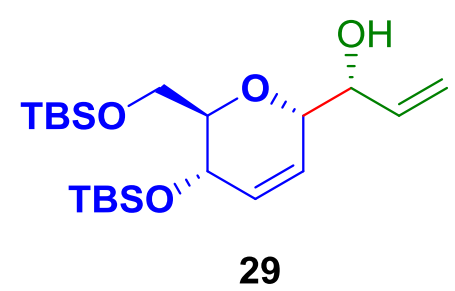

${ }^{13} \mathrm{C}$ NMR (125 MHz, $\mathrm{CDCl}_{3}$ )

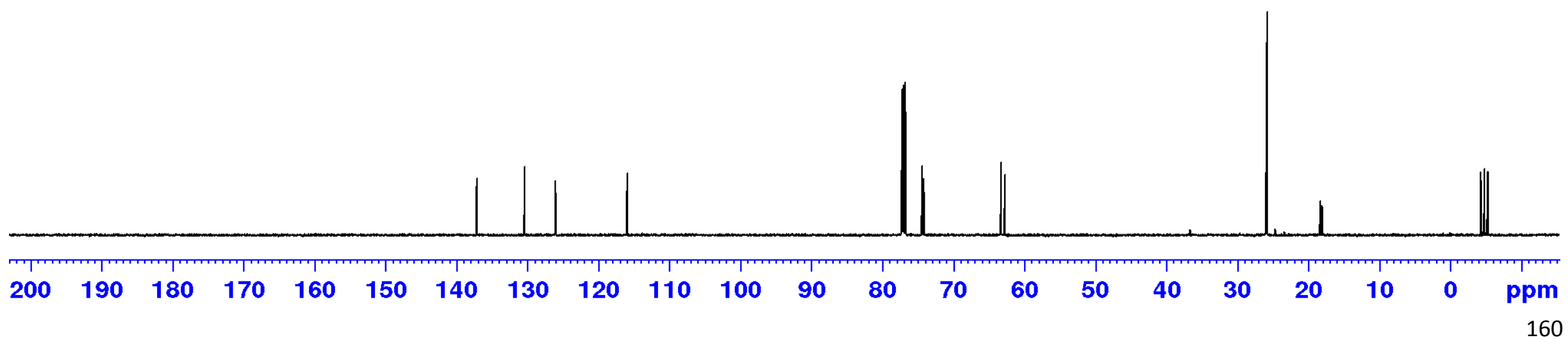



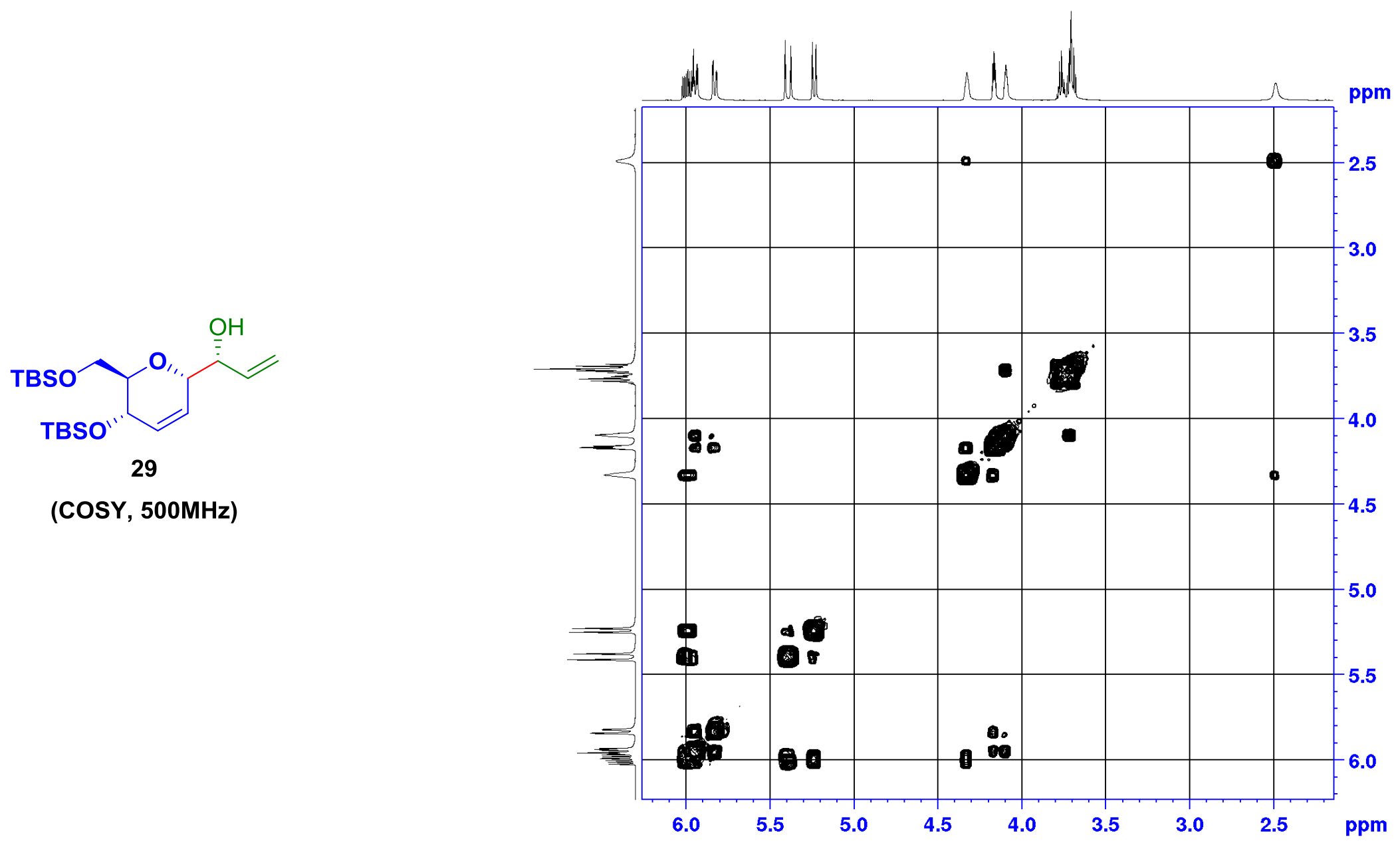


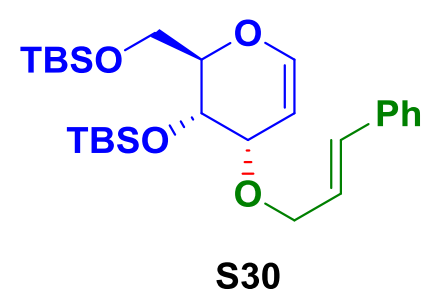

${ }^{1} \mathrm{H}$ NMR (500 MHz, $\mathrm{CDCl}_{3}$ )

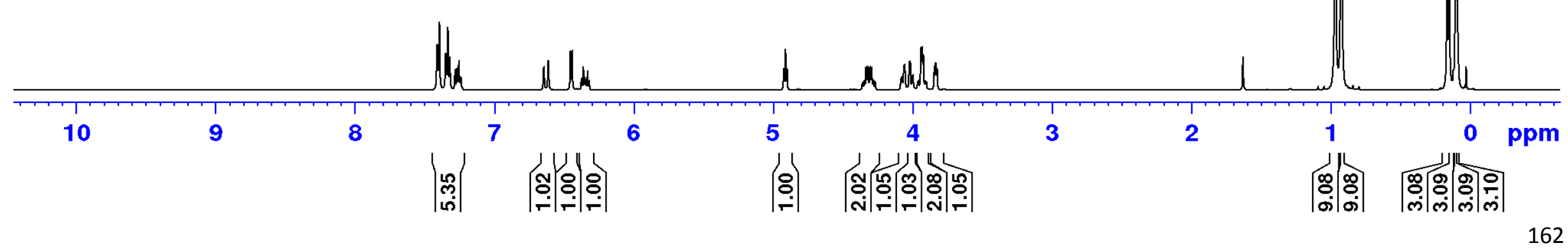



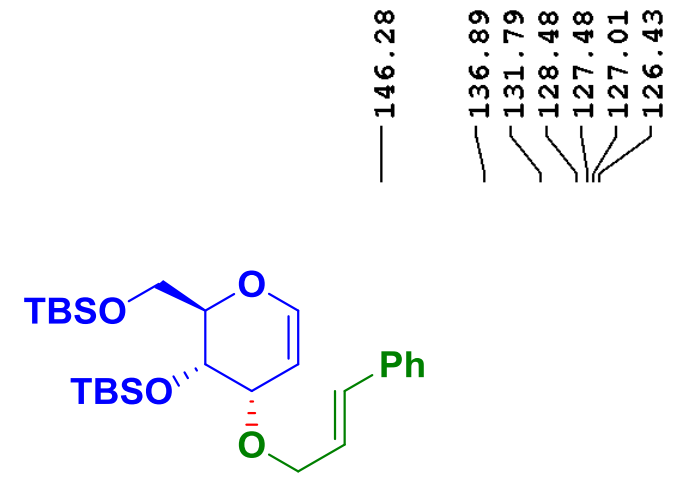

S30

${ }^{13} \mathrm{C}$ NMR (125 MHz, $\mathrm{CDCl}_{3}$ )

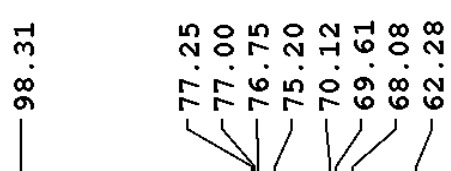

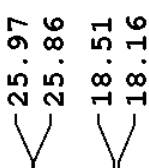

긍용요

†ंगे

î

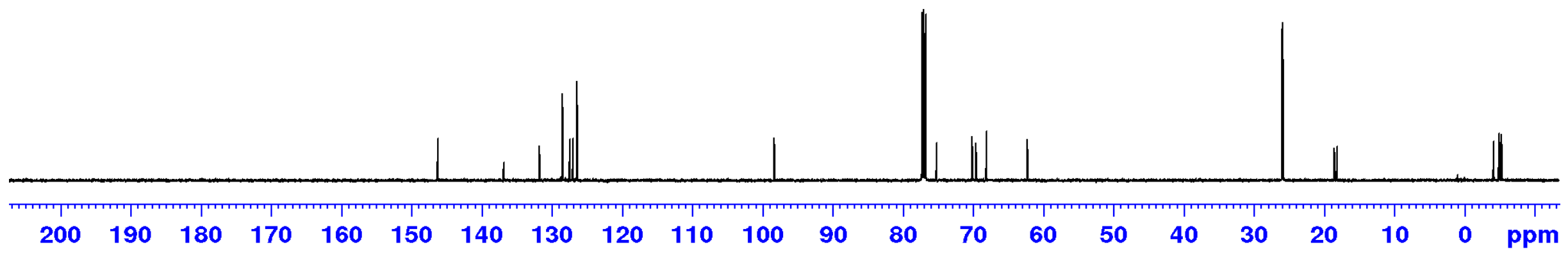




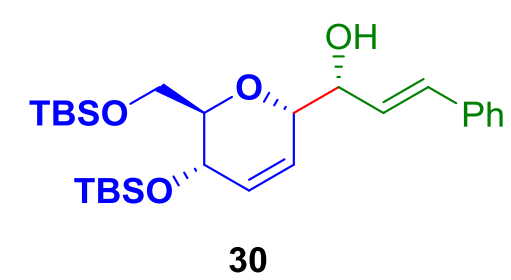

${ }^{1} \mathrm{H}$ NMR (500 MHz, $\left.\mathrm{CDCl}_{3}\right)$

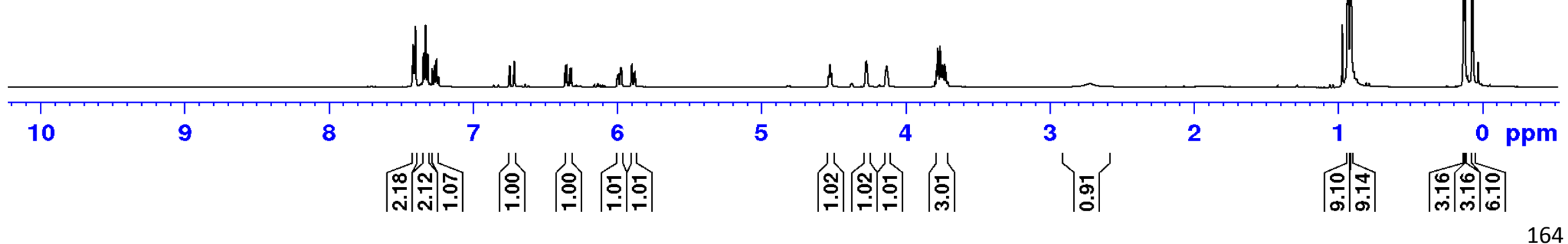




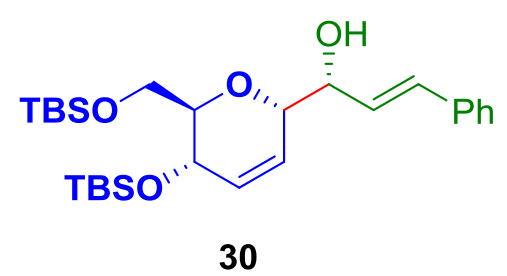

${ }^{13} \mathrm{C}$ NMR (125 MHz, $\mathrm{CDCl}_{3}$ )

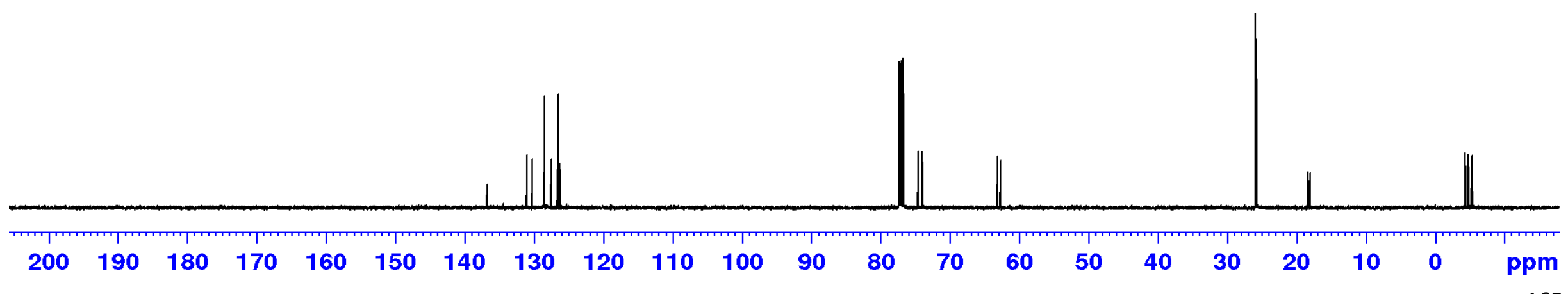



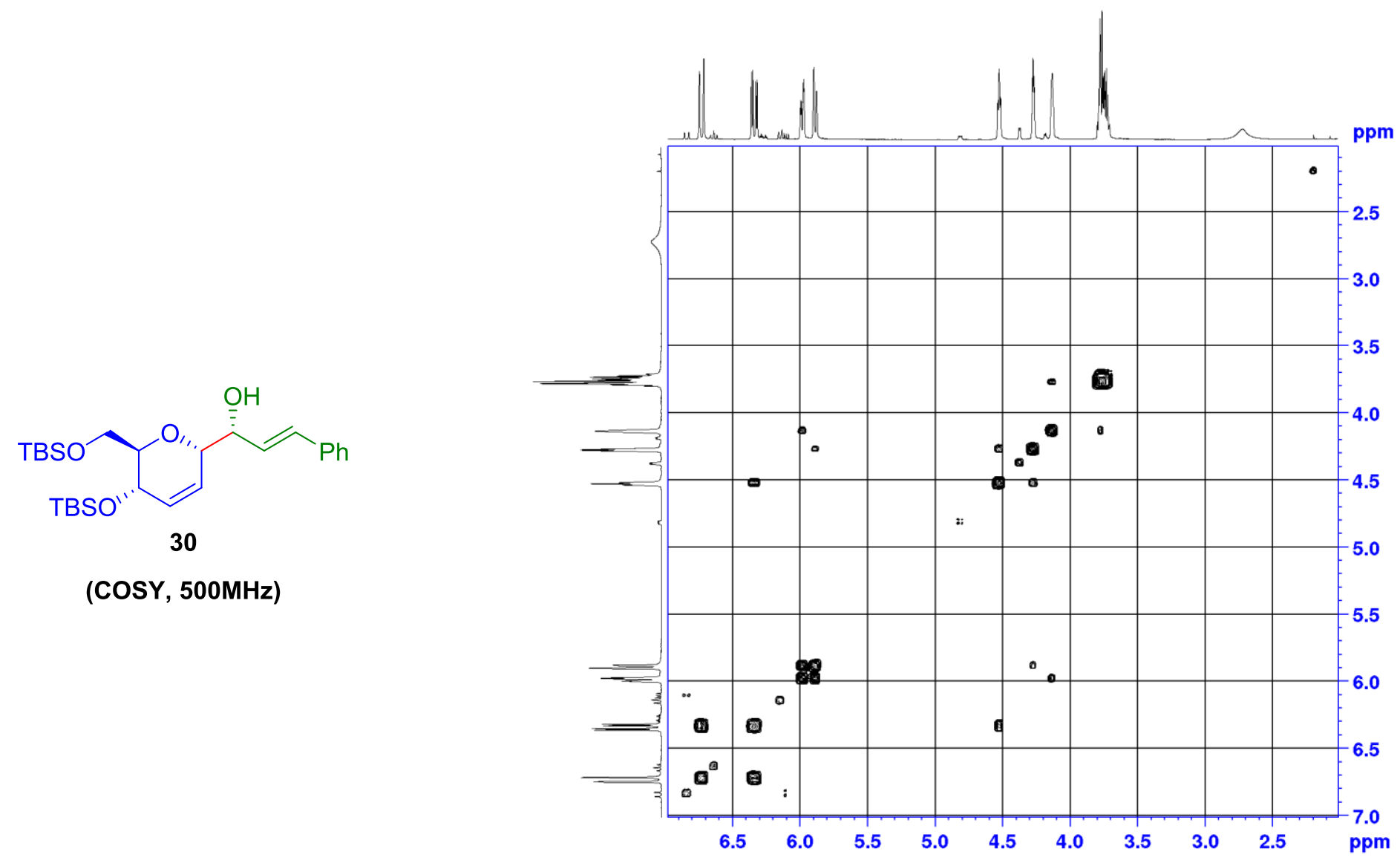


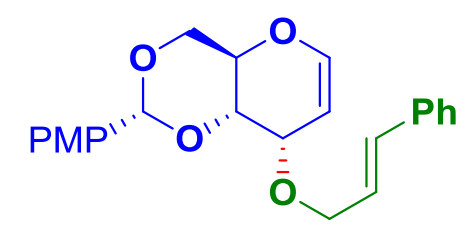

s31

${ }^{1} \mathrm{H}$ NMR (500 MHz, $\mathrm{CDCl}_{3}$ )

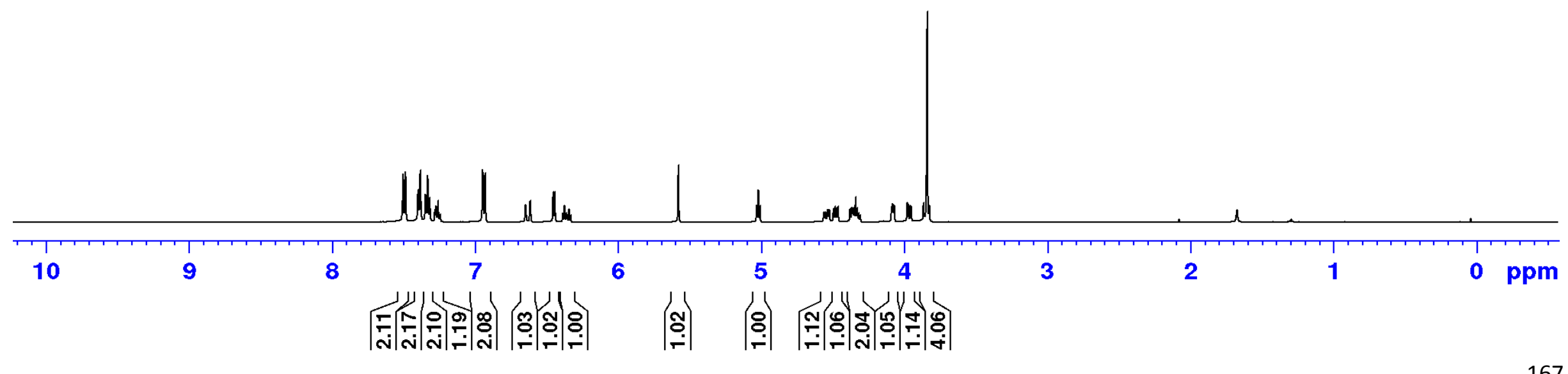




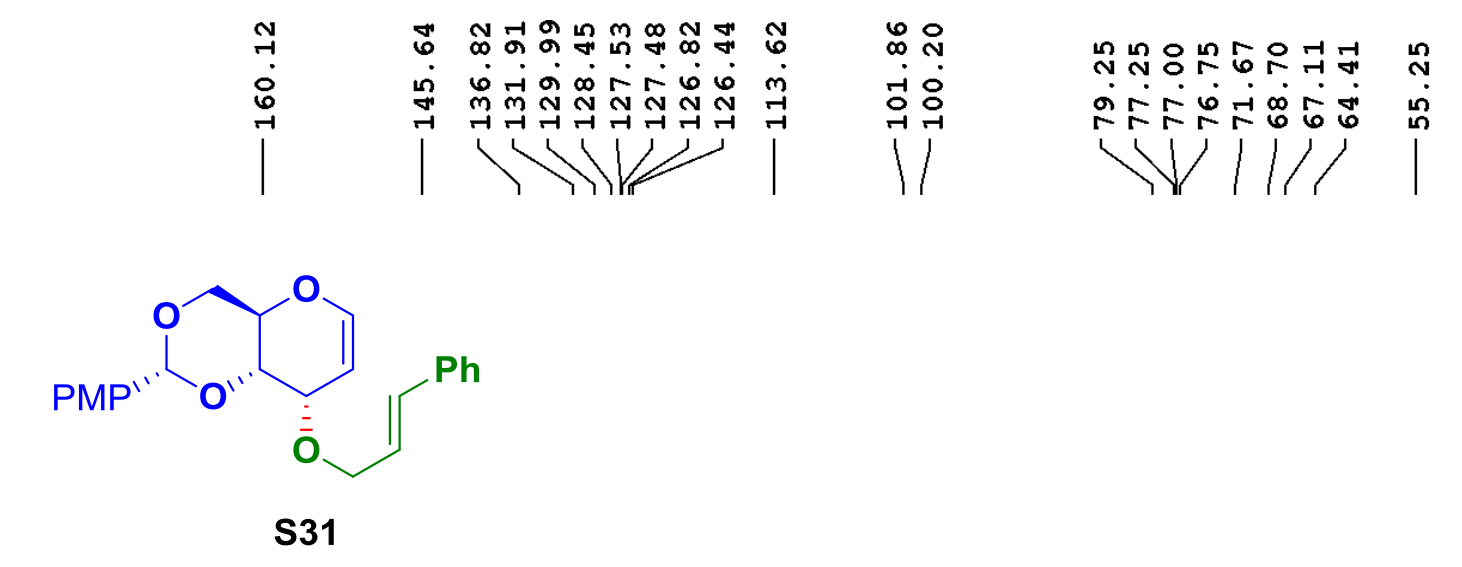

${ }^{13} \mathrm{C}$ NMR (125 MHz, $\mathrm{CDCl}_{3}$ )

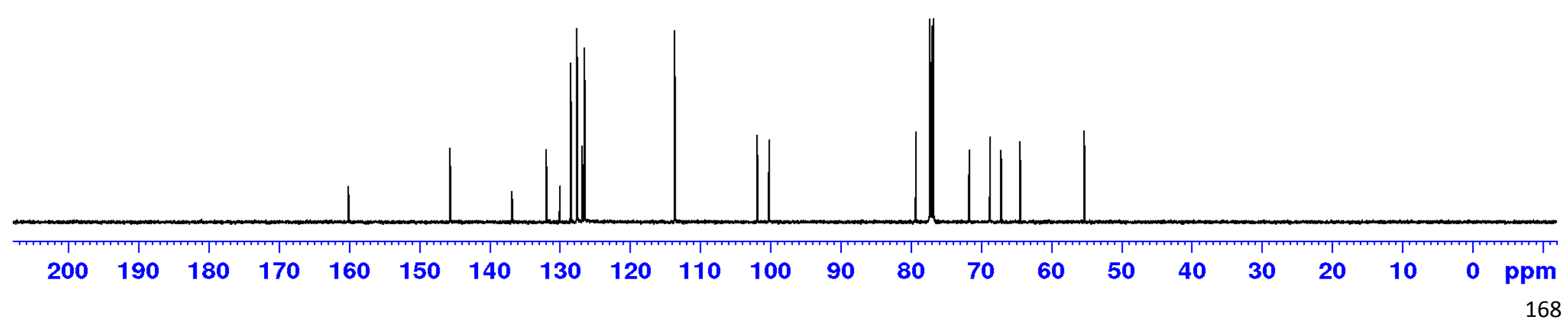




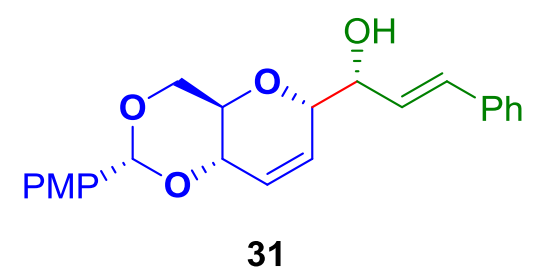

${ }^{1} \mathrm{H}$ NMR (400 MHz, $\mathrm{CDCl}_{3}$ )

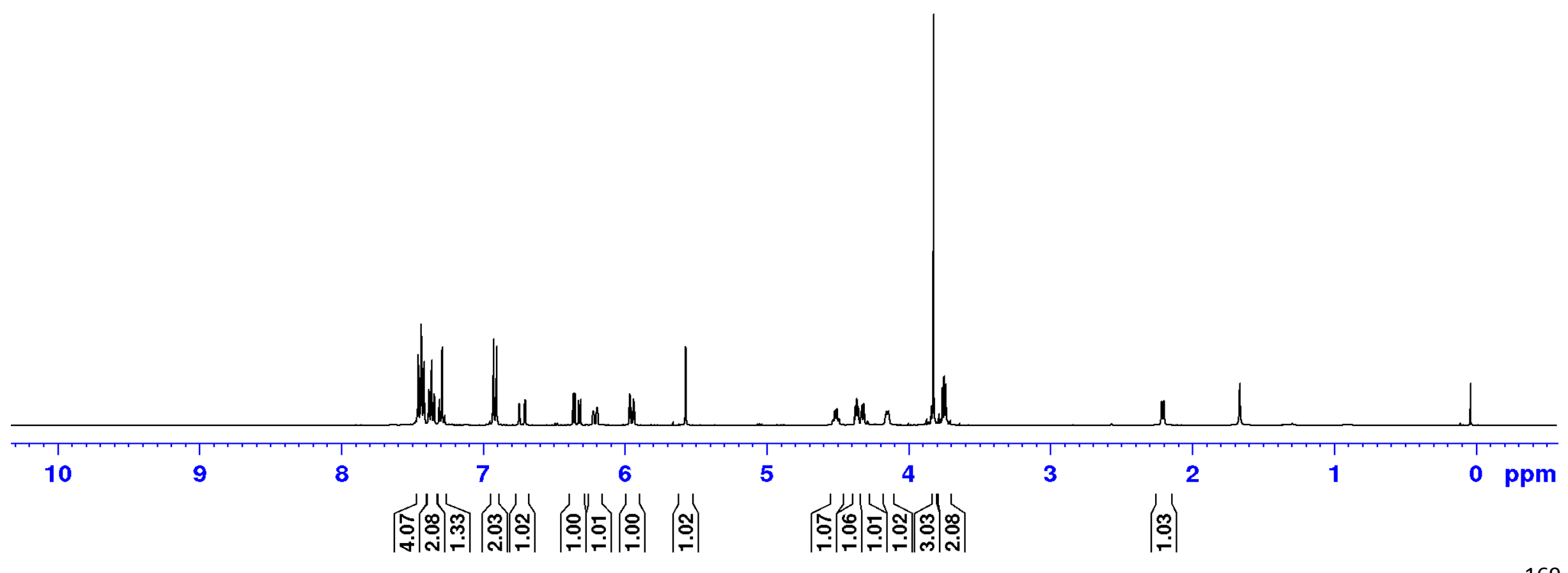


<smiles>CC(C)[Pb]C1OC[C@H]2O[C@H]([C@H](O)/C=C/c3ccccc3)C=C[C@@H]2O1</smiles>

31

${ }^{13} \mathrm{C}$ NMR (100 MHz, $\mathrm{CDCl}_{3}$ )

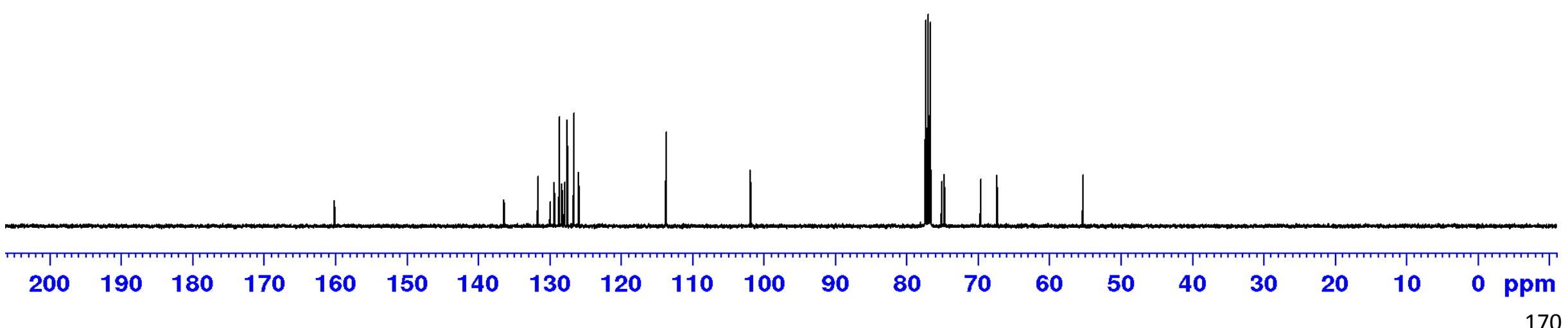



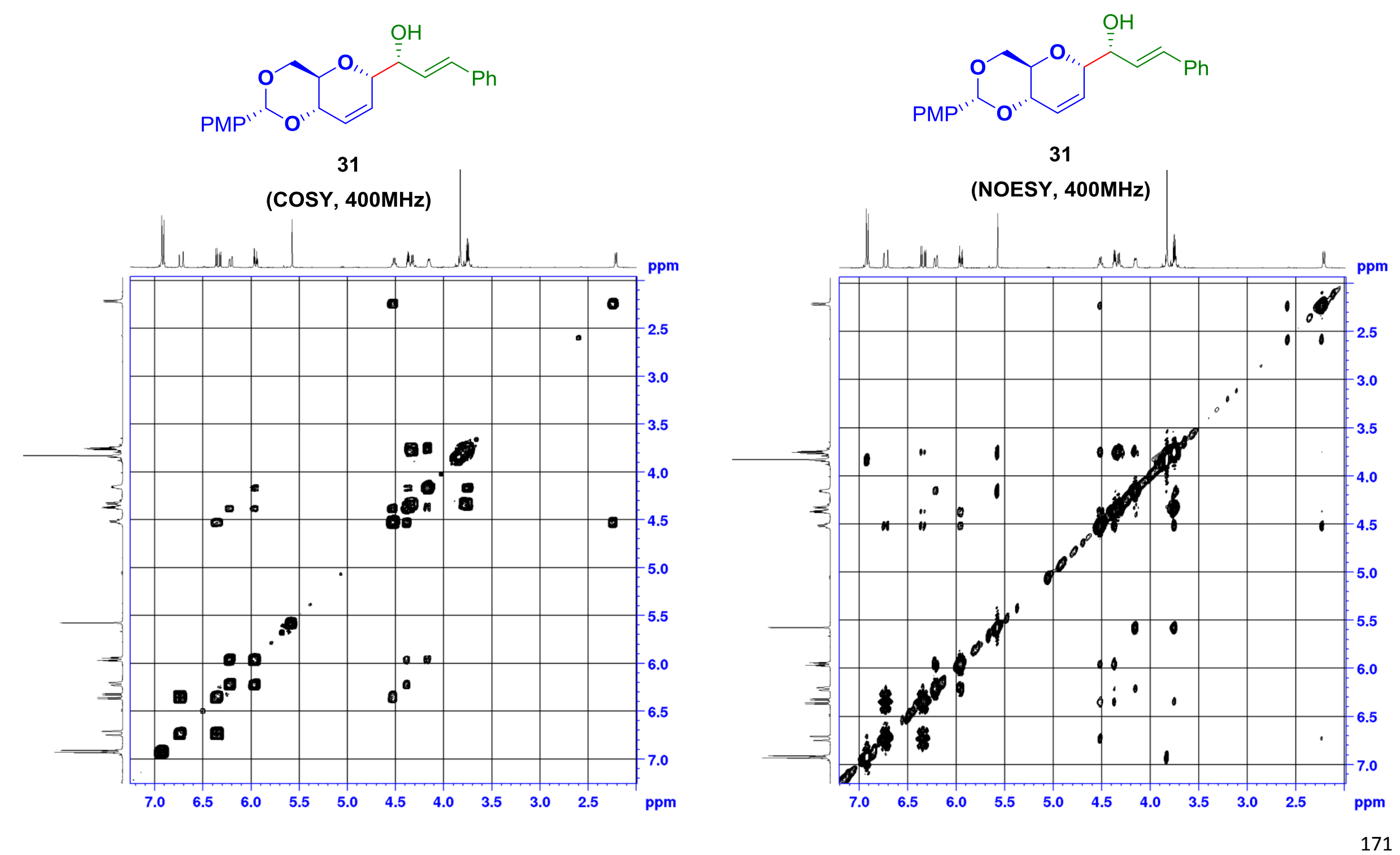


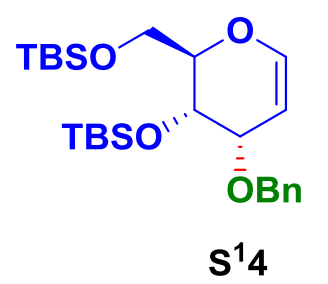

${ }^{1} \mathrm{H}$ NMR (500 MHz, $\mathrm{CDCl}_{3}$ )

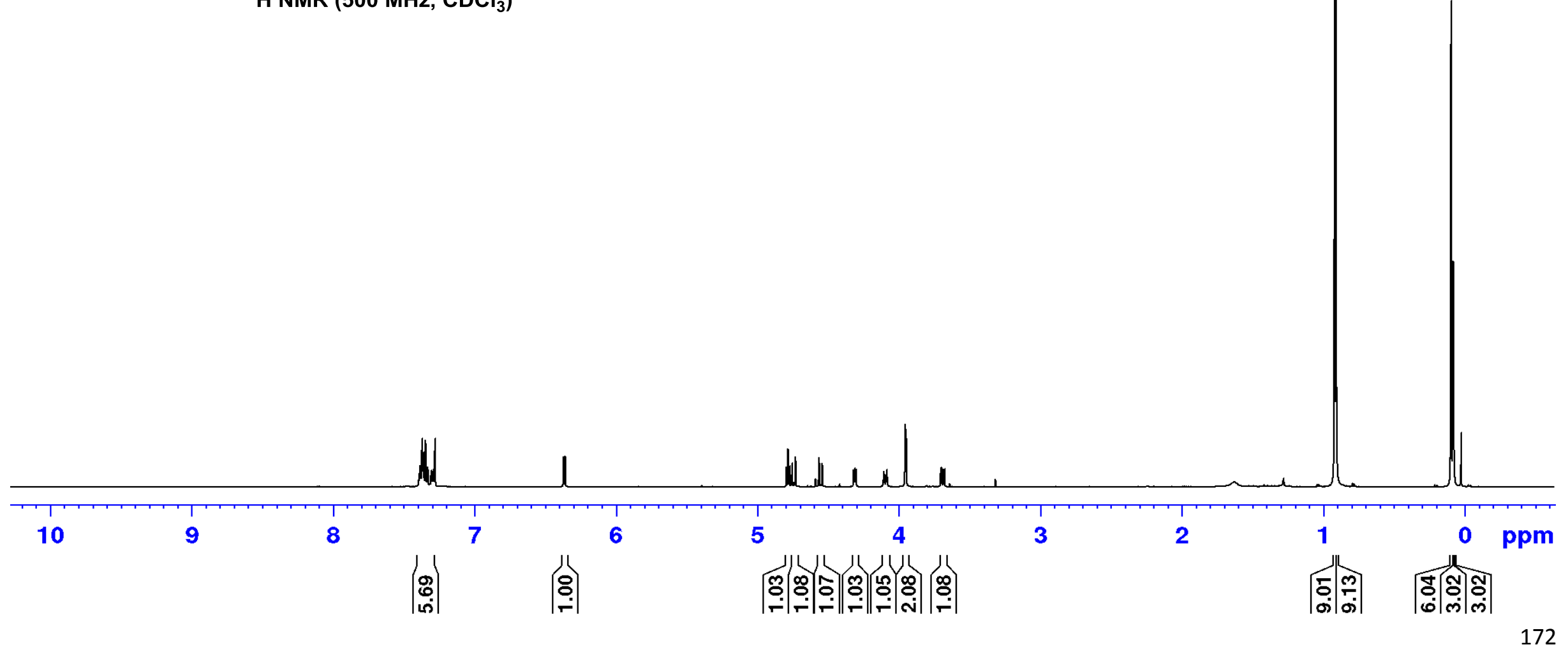



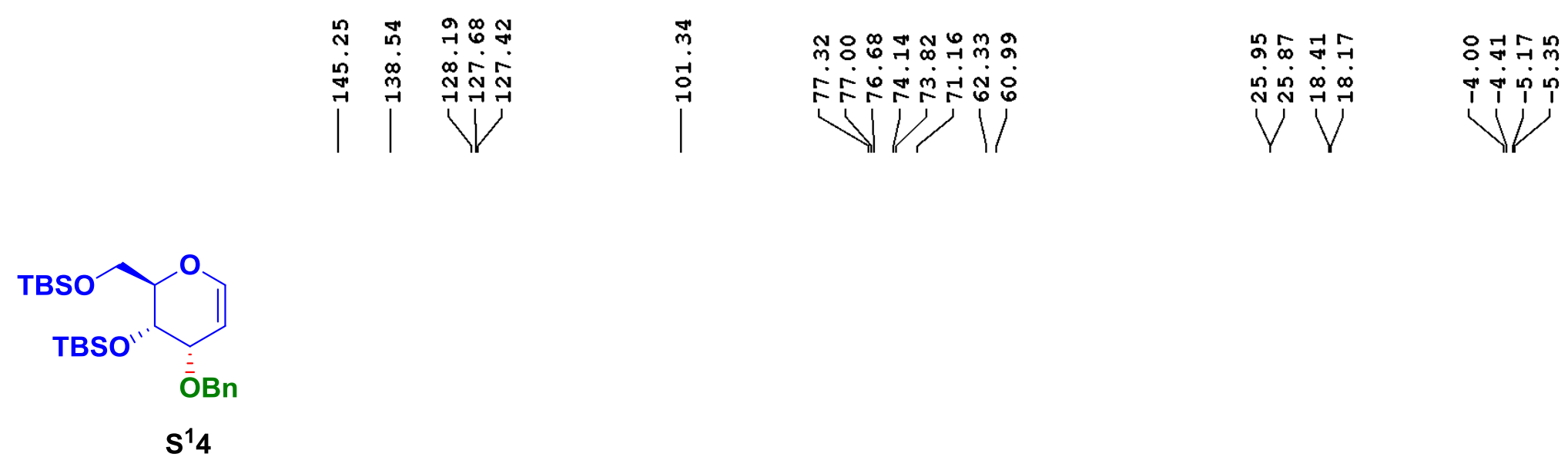

${ }^{13} \mathrm{C}$ NMR (100 MHz, $\mathrm{CDCl}_{3}$ )

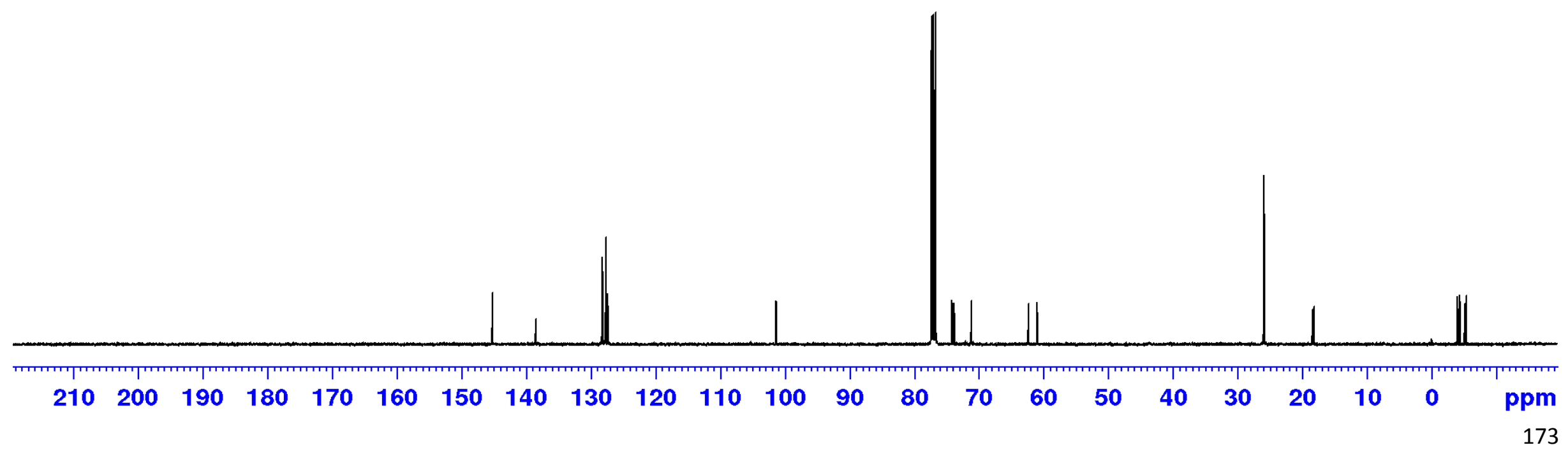




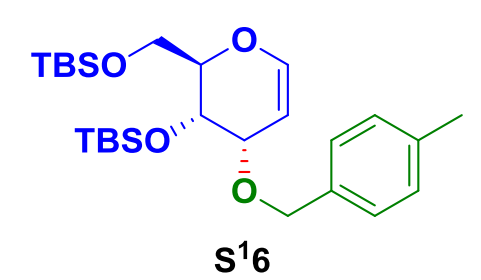

${ }^{1} \mathrm{H}$ NMR (500 MHz, $\mathrm{CDCl}_{3}$ )

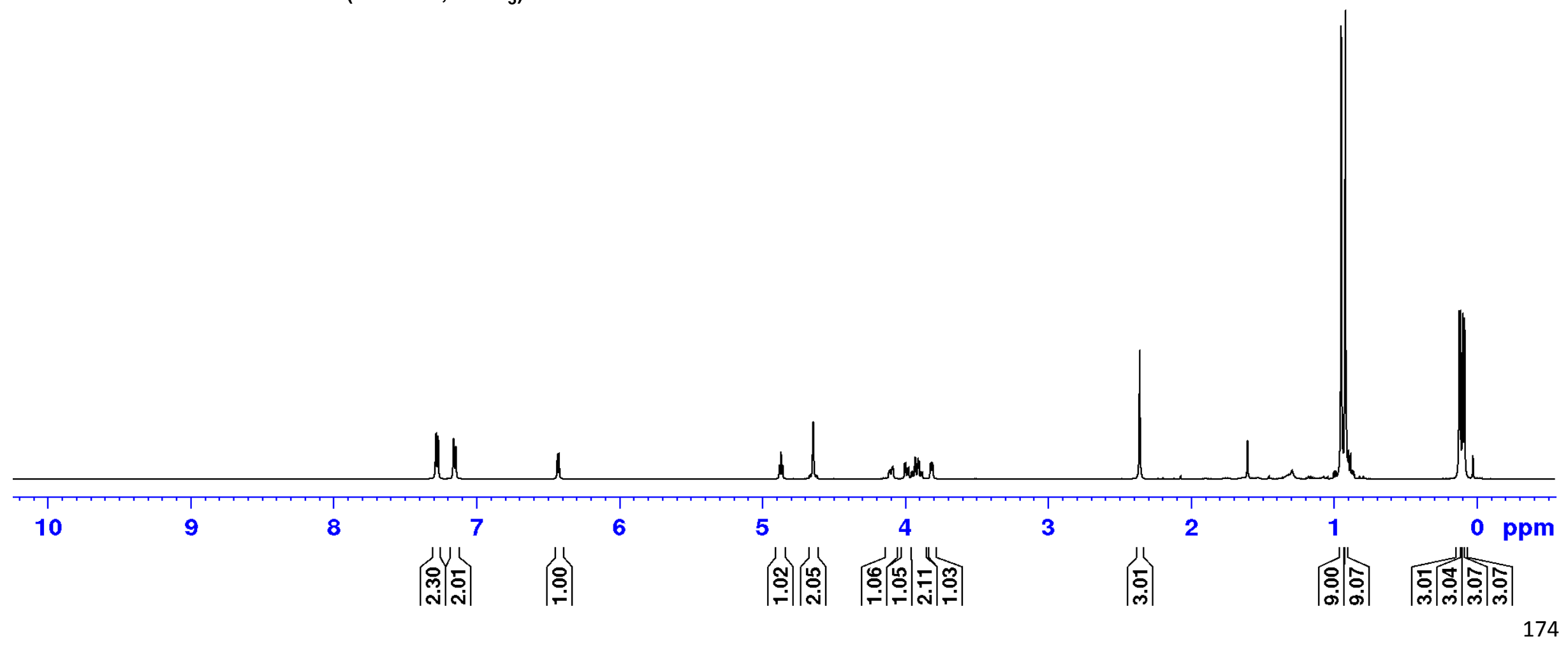



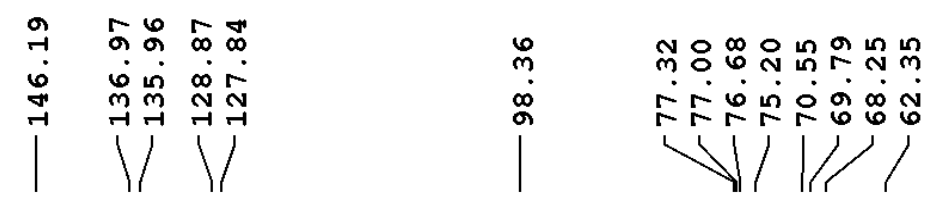

年 국용 -

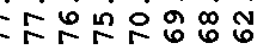

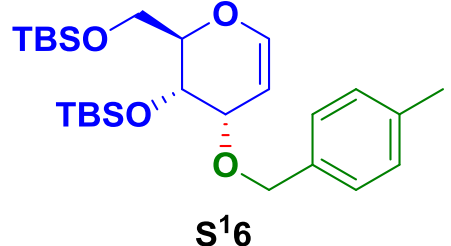

${ }^{13} \mathrm{C}$ NMR (100 MHz, $\mathrm{CDCl}_{3}$ )

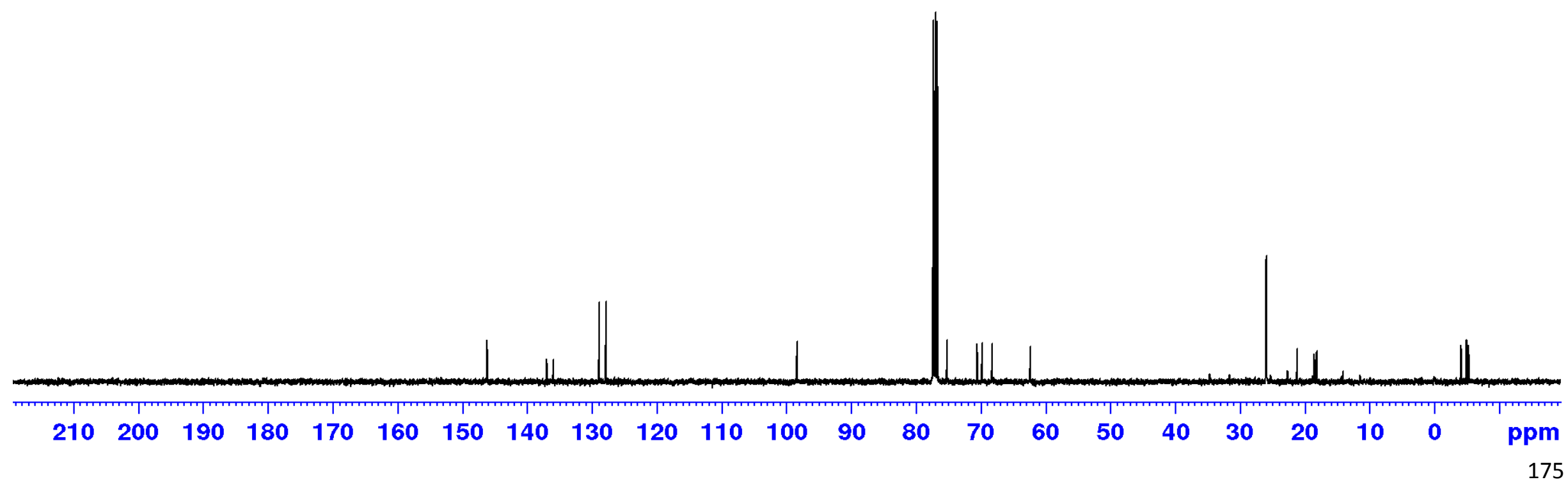




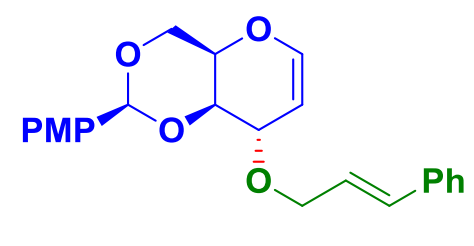

S32

${ }^{1} \mathrm{H}$ NMR (500 MHz, $\mathrm{CDCl}_{3}$ )

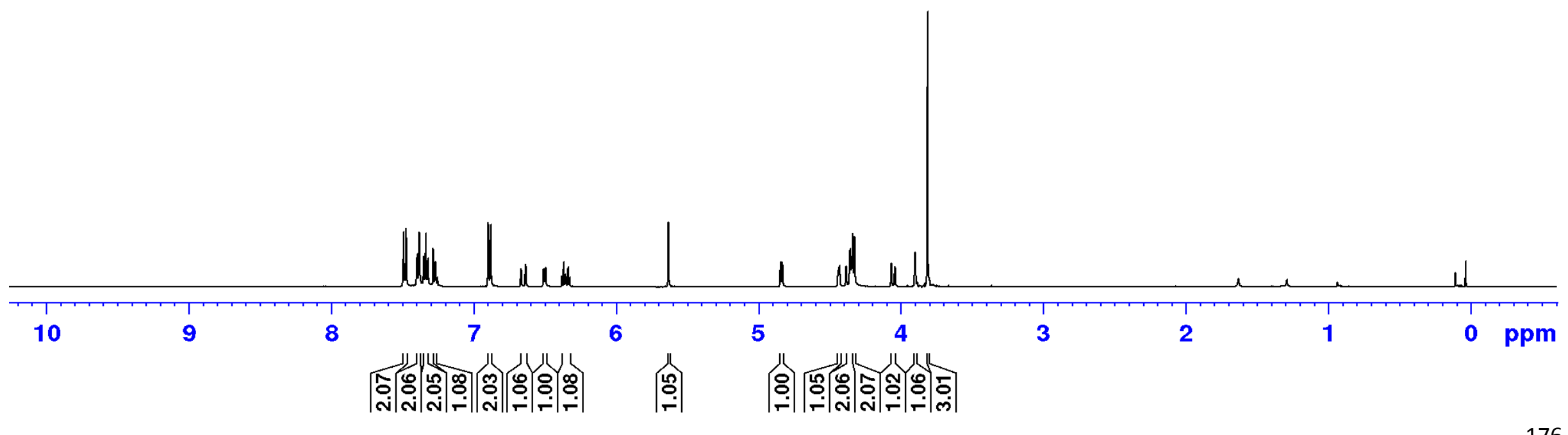



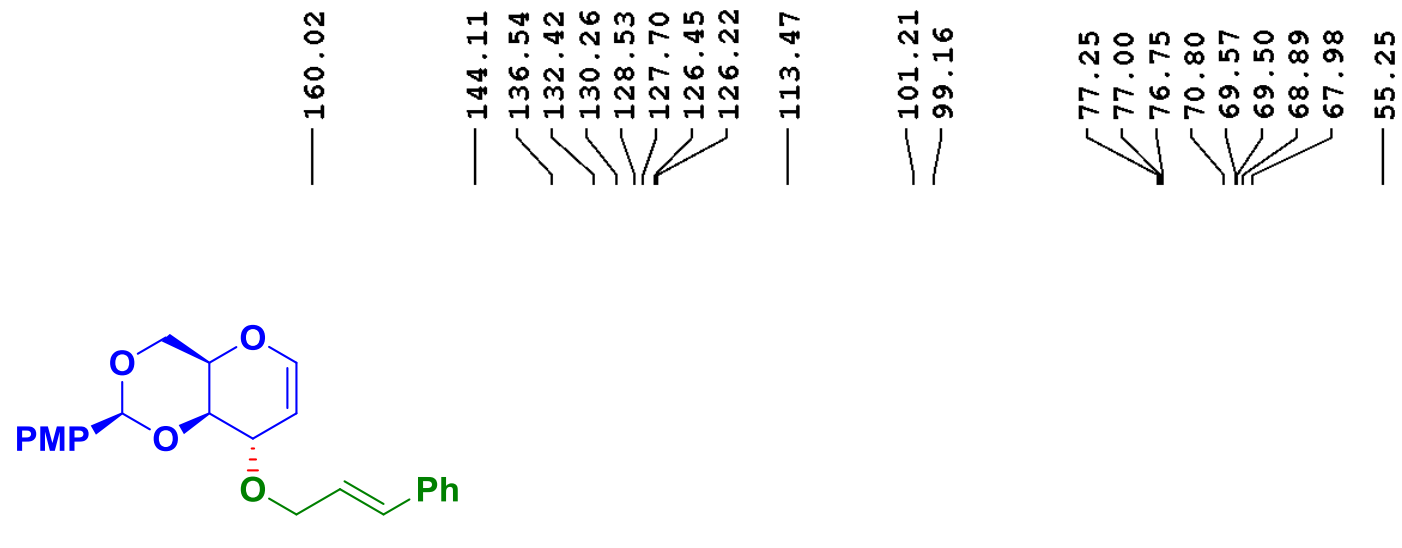

S32

${ }^{13} \mathrm{C}$ NMR (125 MHz, $\mathrm{CDCl}_{3}$ )

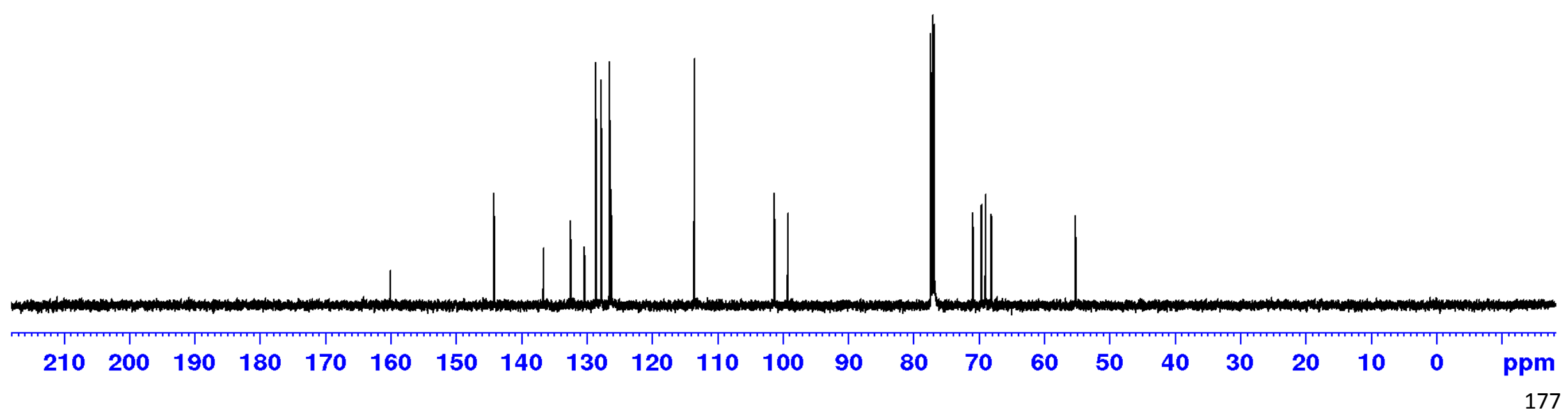




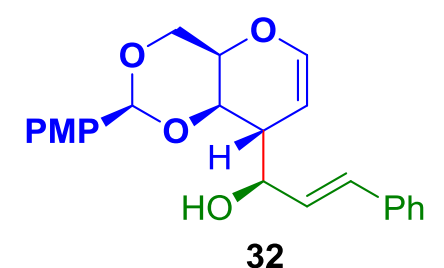

${ }^{1} \mathrm{H}$ NMR (400 MHz, $\mathrm{CDCl}_{3}$ )

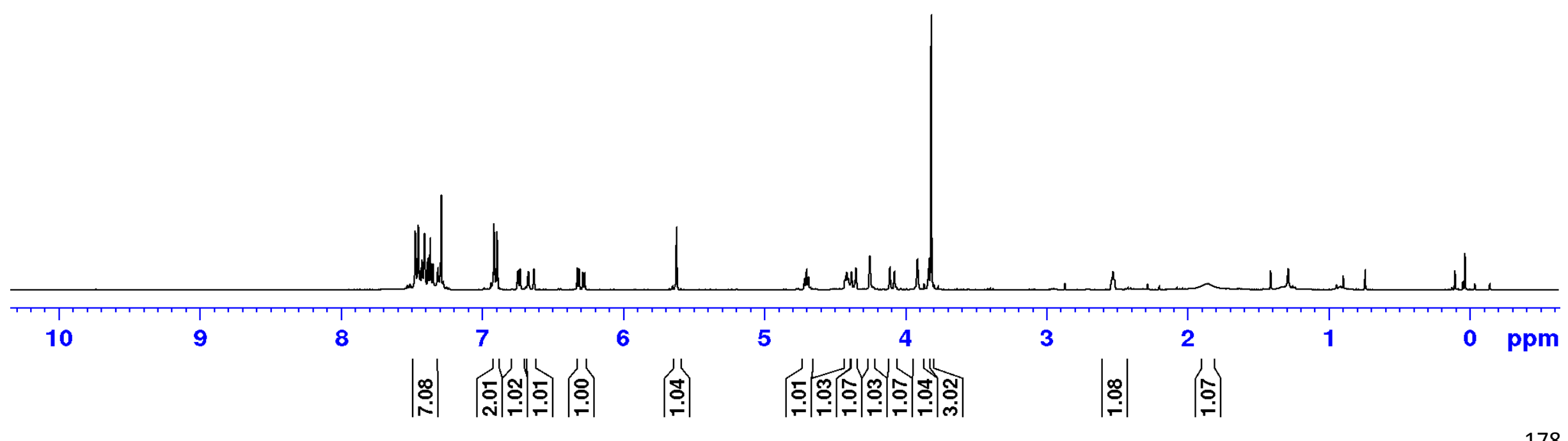


: $\quad$ D

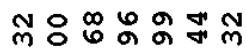

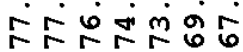

WII

ง

ถึก กู

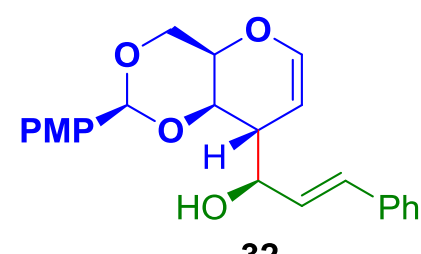

32

${ }^{13} \mathrm{C}$ NMR (100 MHz, $\left.\mathrm{CDCl}_{3}\right)$

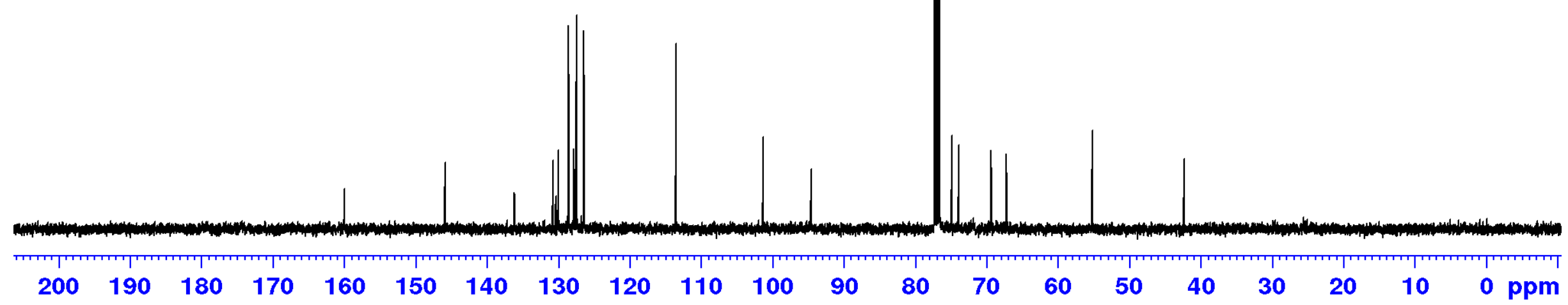

